\title{
Type IIB Orientifolds, D-Brane Instantons And The Large Volume Scenario
}

\author{
Erik Plauschinn
}

\author{
Dissertation \\ an der Fakultät für Physik \\ der Ludwig-Maximilians-Universität \\ München
}

vorgelegt von

Erik Plauschinn

aus Dresden

München, 2009 
Erstgutachter:

Zweitgutachter:

Tag der mündlichen Prüfung:
Prof. Dr. Dieter Lüst

Ludwig-Maximilians-Universität München Max-Planck-Institut für Physik, München

Priv.-Doz. Dr. Ralph Blumenhagen Max-Planck-Institut für Physik, München 28.07.2009 


\section{Table of Contents}

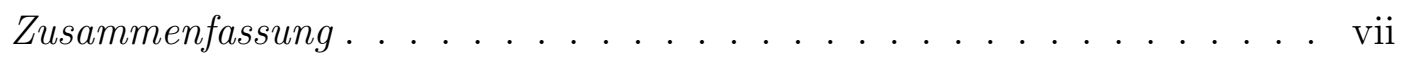

Summary ................................ ix

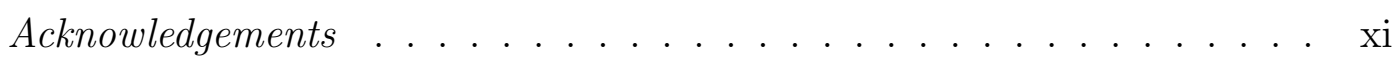

\begin{tabular}{ll}
\hline Introduction & $\mathbf{1}$
\end{tabular}

\begin{tabular}{lll}
\hline & Introduction & 3 \\
\hline
\end{tabular}

1.1 Why String Theory? . . . . . . . . . . . . . . . . . . . . . . . . . . . . . . 3

1.2 String Phenomenology . . . . . . . . . . . . . . . . . . . . . . . . . . . . . . . . . . . . 2

1.3 Overview . . . . . . . . . . . . . . . . . . . . . . . 20

\begin{tabular}{lll}
\hline II & Prerequisites & 23
\end{tabular}

2 Orientifold Compactifications and Effective Actions 25

2.1 Orientifold Compactifications . . . . . . . . . . . . 25

2.2 On the Effective Action for the Closed String Sector . . . . . . . . . . 29

2.3 On the Effective Action for the Open String Sector . . . . . . . . . 32

$\begin{array}{lll}3 & \mathcal{N}=1 \text { Supergravity Description } & 37\end{array}$

3.1 Generalities . . . . . . . . . . . . . . . . . . 37

3.2 Type IIB Orientifolds with O3- and O7-Planes . . . . . . . . . . . . 39

3.2 .1 The Kinetic Part . . . . . . . . . . . . . . . . . . . . . . . . 39

3.2 .2 The Kähler Potential and the Kähler Metric . . . . . . . . . 39

3.2 .3 The Superpotential . . . . . . . . . . . . . . . . . . . . . . 43

3.2 .4 The Gauge Kinetic Function . . . . . . . . . . . . . . . . . . 44 
4 Aspects of String Theory Model Building 47

4.1 Overview . . . . . . . . . . . . . . . . . . . . . . 47

4.2 Chiral Spectrum . . . . . . . . . . . . . . . . . . . . . . . . . 48

4.3 The Generalised Green-Schwarz Mechanism . . . . . . . . . . . . . 50

4.4 Supersymmetry Conditions . . . . . . . . . . . . . . . . . 50

4.5 Freed-Witten Anomaly Cancellation . . . . . . . . . . . . . . . . . . 51

4.6 K-Theory Constraints . . . . . . . . . . . . . . . . . . . 52

$\begin{array}{lll}5 & \text { D-Brane Instantons } & 53\end{array}$

5.1 Prerequisites . . . . . . . . . . . . . . . . . . 53

5.2 Instanton Calculus $\ldots \ldots \ldots \ldots$. . . . . . . . . . . 56

$\begin{array}{llr}6 & \text { Moduli Stabilisation } & 61\end{array}$

6.1 The KKLT Scenario . . . . . . . . . . . . . . . . . . . . . . . . . 61

6.1 .1 General Analysis . . . . . . . . . . . . . . . . 61

6.1 .2 An Example . . . . . . . . . . . . . . . . . . . . . 65

6.1 .3 Discussion . . . . . . . . . . . . . . . . . . . . . . 67

6.2 The LARGE Volume Scenario . . . . . . . . . . . . . . . . . . . 68

6.2 .1 General Analysis . . . . . . . . . . . . . . . . . . . 69

6.2 .2 An Example . . . . . . . . . . . . . . . . . . . . . . . . 74

$6.2 .3 \quad$ Discussion . . . . . . . . . . . . . . . . . . . . . . . . . . . 77

\begin{tabular}{llr}
\hline III Developments & 81
\end{tabular}

7 The Generalised Green-Schwarz Mechanism 83

7.1 Tadpole Cancellation Conditions _ . . . . . . . . . . . . . . 83

7.1.1 Variation of the Action . . . . . . . . . . . . . . . . . . . . 84

7.1.2 Tadpole Cancellation Conditions . . . . . . . . . . . . . 86

7.2 The Generalised Green-Schwarz Mechanism . . . . . . . . . . . . . 90

7.2 .1 Chiral Anomalies . . . . . . . . . . . . . . . . . . . . 91

7.2 .2 Green-Schwarz Couplings . . . . . . . . . . . . . . . . 93

$7.2 .3 \quad$ Green-Schwarz Diagrams . . . . . . . . . . . . . . . . . . . . 96

7.2.4 Massive U(1)s and Fayet-Iliopoulos Terms . . . . . . . . . . 98

7.3 Including D9- and D5-Branes for the O3-/O7-System . . . . . . . . 100

7.3.1 Tadpole Cancellation Conditions . . . . . . . . . . . . . 100

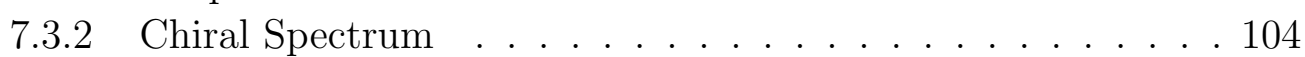

7.3 .3 Chiral Anomalies . . . . . . . . . . . . . . . . . . . . . 106

7.3.4 Massive U(1)s and Fayet-Iliopoulos Terms . . . . . . . . . . 107

7.4 Summary . . . . . . . . . . . . . . . . . . 110 
$8 \quad$ Applications of the D-Brane Instantons Calculus 113

8.1 The ADS Superpotential . . . . . . . . . . . . . . . . . . . . . 113

8.2 A Chiral Zero-Mode Constraint . . . . . . . . . . . . . . . . . . . 119

8.3 Summary . . . . . . . . . . . . . . . . . . . . . . . . . . . . . . 124

9 The LARGE Volume Scenario with MSSM Sector 125

9.1 General Analysis . . . . . . . . . . . . . . . . . . . . 125

9.2 An Example: The $\mathbb{P}_{[1,3,3,3,5]}[15]$ Calabi-Yau $\ldots$. . . . . . . . . . . . . 130

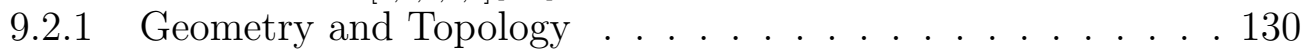

9.2 .2 Constructing the Open String Sector . . . . . . . . . . . . . 133

9.2 .3 Moduli Stabilisation . . . . . . . . . . . . . . . . . 136

9.2 .4 Discussion . . . . . . . . . . . . . . . . . . . . . . . . . . . . 139

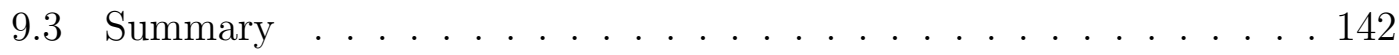

\begin{tabular}{lll}
\hline IV Summary and Outlook & 147
\end{tabular}

\begin{tabular}{lr}
\hline 0 Summary and Outlook & 149
\end{tabular}

10.1 Summary . . . . . . . . . . . . . . . . . . . . . . . . . . . . . . . . 149

10.2 Outlook . . . . . . . . . . . . . . . . . . . 152

\begin{tabular}{lll}
\hline Appendices & 155
\end{tabular}

\begin{tabular}{|ll}
\hline A Definition of Topological Quantities & 157
\end{tabular}

\begin{tabular}{ll}
\hline B Details on the Kähler Metric & 161
\end{tabular}

C The Gukov-Vafa-Witten Superpotential 165

D Chiral Anomalies for Gauge Groups $\mathrm{SO}(2 \mathrm{~N})$ and $\operatorname{Sp}(2 \mathrm{~N}) \quad 169$

\begin{tabular}{|lll}
\hline T The Chiral Zero-Mode Constraint for $\mathrm{h}_{-}^{1,1} \neq 0$ & 171
\end{tabular}

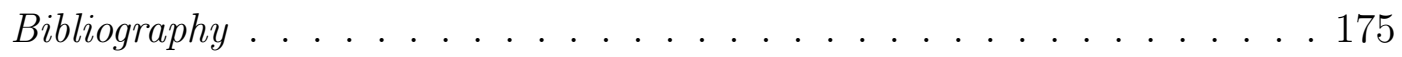




\section{Zusammenfassung}

Diese Dissertation befasst sich mit einem Forschungsgebiet in der String Theorie genannt String Phänomenologie, in welchem die Verbindung zwischen String Theorie und der bekannten Hochenergiephysik untersucht wird. Drei Themengebiete, welche für solch eine Verbindung wichtig sind, werden in dieser Arbeit genauer behandelt.

Das erste Gebiet befasst sich mit der Konstruktion von String Theorie Modellen im Kontext von sogenannten Typ IIB Orientifolds mit Orientifold Drei- und Sieben-Ebenen. Wir untersuchen eine wichtige Konsistenzbedingung für solche Konstruktionen, die sogenannte Tadpole Kürzungsbedingung, und verifizieren, dass chirale Anomalien durch den verallgemeinerten Green-Schwarz Mechanismus gekürzt werden.

Das zweite Gebiet dieser Arbeit befasst sich mit nicht-perturbativen Effekten in Typ II String Konstruktionen, sogenannten D-Brane Instantonen. Wir diskutieren den Instanton Kalkül, leiten das sogenannte Affleck-Dine-Seiberg Superpotential in String Theorie her und entwickeln unter Berücksichtigung eines realistischen Teilchenphysik Sektors eine chirale Null-Moden Bedingung für den Beitrag von D-Brane Instantonen zum Superpotential.

Im dritten Gebiet dieser Arbeit diskutieren wir Moduli Stabilisierung in Typ IIB String Kompaktifizierungen. Wir erklären das sogenannte KKLT Szenario sowie das Large Volume Scenario; und wir konstruieren und untersuchen ein Modell für das Large Volume Scenario in welchem die chirale Null-Moden Bedingung berücksichtigt wird.

Obwohl die drei Themengebiete dieser Dissertation etwas verschieden voneinander sind, existiert jedoch zwischen ihnen kompliziertes Zusammenspiel mit vielen Wechselbeziehungen. Um diese Zusammenhänge zu studieren, wurden die einzelnen Gebiete genau untersucht und miteinander verknüpft. Dies hat zu neuen Resultaten, wie zum Beispiel der oben erwähnten chiralen Null-Moden Bedingung, geführt. 


\section{Summary}

This thesis is concerned with a branch of research in String Theory called String Phenomenology which aims for a better understanding of the connection between String Theory and Particle Physics. In particular, in this work we cover three topics which are important in order to establish this connection.

The first topic is about String Theory model building in the context of so-called type IIB orientifolds with orientifold three- and seven-planes. After giving a brief overview, we work out in detail an important consistency condition for String Theory constructions, the so-called tadpole cancellation condition, and we verify explicitly that chiral anomalies are cancelled via the generalised Green-Schwarz mechanism.

The second topic is concerned with so-called D-brane instantons which are nonperturbative effects in type II String Theory constructions. We recall the instanton calculus for such configurations, we derive the so-called Affleck-Dine-Seiberg superpotential in String Theory and we develop an important constraint, a chiral zero-mode constraint, for instanton contributions in the presence of a realistic Particle Physics sector.

The third topic is about moduli stabilisation in type IIB string compactifications. More concretely, we review the so-called KKLT as well as Large Volume Scenario, and we construct and study a model for the latter scenario where the constraint mentioned above has been taken into account explicitly.

Although the three topics studied in this thesis are slightly different in nature, there is nevertheless a complex interplay between them with many interrelations. In order to uncover these connections, a detailled study of each individual subject has been performed which has led to new results such as the chiral zero-mode constraint. 


\section{Acknowledgements}

First of all, I would like to express my special gratitude to Dieter Lüst and Ralph Blumenhagen for giving me the opportunity to work on String Theory under their supervision. I am deeply indebted to them for sharing their knowledge, insight and experience on this subject as well as for constantly supporting, encouraging and stimulating me during the period of my Ph.D.

I also want to thank Nikolas Akerblom, Sebastian Moster and Maximilian SchmidtSommerfeld not only for the fruitful collaborations and extremely helpful discussions, but also for providing a very enjoyable and pleasant atmosphere at the Max-Planck-Institute for Physics.

I furthermore want to thank - in alphabetical order - Martin Ammon, Johannes Brödel, Florian Gmeiner, Daniel Härtl, Benjamin Jurke, Matthias Kaminski, Johanna Knapp, Michael Haack, Robert Richter, Felix Rust, Stephan Stieberger, Christoffer Petersson, Michael Plümacher, Jochen Schieck, Timo Weigand and Marco Zagermann for many interesting discussion, for their patience in answering my questions and for a very enjoyable time. Special thanks are due to Marco Zagermann for agreeing to write a Letter of Recommendation supporting my PostDoc applications.

I would also like to thank the members of the String Theory group in Munich and the members of the Max-Planck-Institute for Physics for providing an inspiring working atmosphere as well as a pleasent environment.

Finally, I would like to thank my friends, my sister, my parents and my girlfried for their support, love and understanding. 


\section{Part I}

\section{Introduction}





\section{Chapter 1}

\section{Introduction}

\subsection{Why String Theory?}

\section{A Brief History of Unification in Physics}

In order to approach the question why one should study String Theory, let us consider the illustration shown in figure 1.1 and briefly review how in the past unification has led to a better understanding of physics [1].

- Even though the interplay between Electricity and Magnetism had already been investigated and partially formulated by Ørsted, Ampère and Faraday, it was Maxwell who brought the theory of Electromagnetism into its final and complete form. In particular, in 1862 he published a set of equations, later called Maxwell equations, which allowed him to derive the electromagnetic wave equation in 1864.

From this example we see that the unified theory of Electromagnetism has led to novel insight into physics, namely the prediction of electromagnetic waves.

- In applying the concept of Galilean Relativity to the theory of Electromagnetism, in the years 1904 and 1905, Lorentz, Poincaré and Einstein formulated Special Relativity which is a refinement of the Galilean principle. Furthermore, Einstein emphasised that in the context of Special Relativity the speed of light is universal and so he argued against the notion of absolute space and time.

Here we see again that unifying different physical ideas has led to a better understanding of physics. 


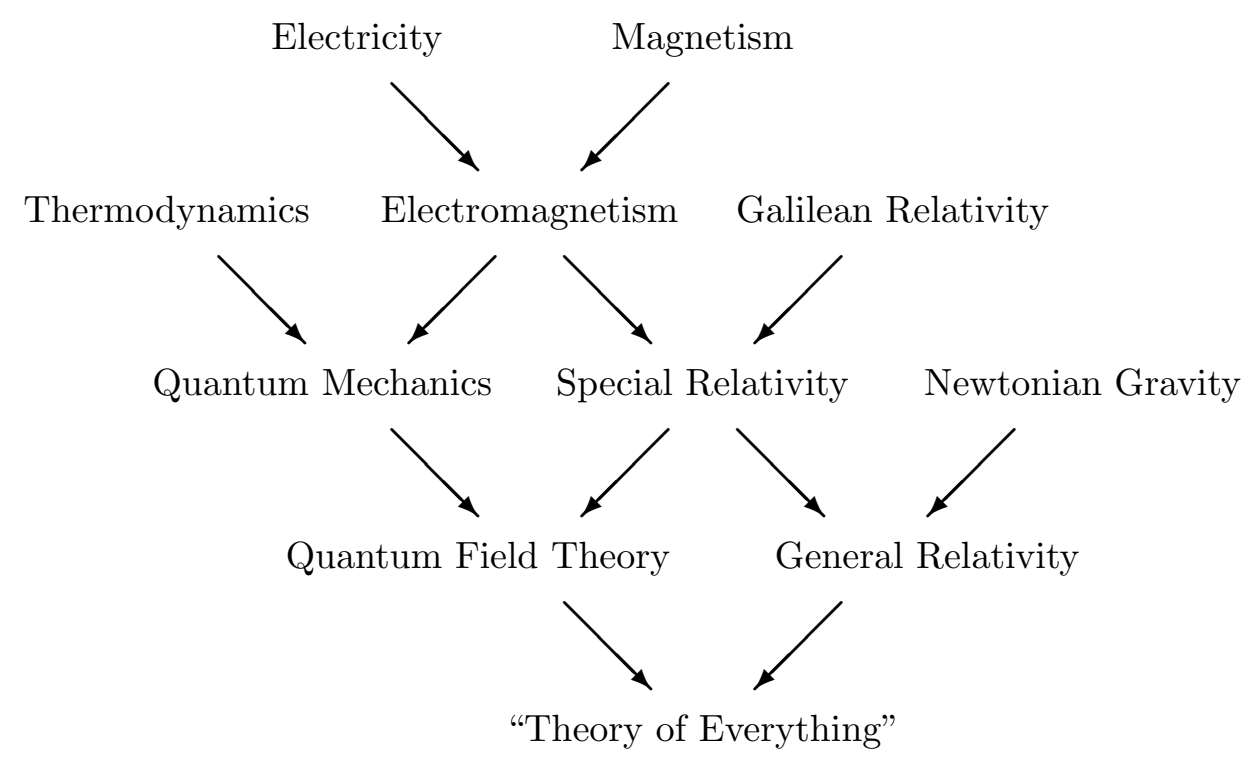

Figure 1.1: Illustration of how unification in physics has led to novel insight $[2,3]$.

- In 1900, by combining the notion of entropy with the concept of electromagnetic waves, Planck was able to derive the spectral radiance of electromagnetic radiation emitted by a black body for all frequencies. However, in order to do so, he had to require the energy to be quantised which eventually led to the development of Quantum Mechanics by Heisenberg, Schrödinger and others.

From this perspective, the development of Quantum Mechanics is therefore linked to the combination of Thermodynamics and Electromagnetism.

- In 1927, it was Dirac who started the process of unifying Quantum Mechanics and Special Relativity by developing a relativistic description of the electron. During the 1940s, following the work of Dirac and others, Feynman, Dyson, Schwinger and Tomonaga invented renormalisation which gave rise to the formulation of Quantum Electrodynamics being the first example of a modern Quantum Field Theory.

Subsequent work of many other physicists then culminated in the so-called "Standard Model of Particle Physics" which is a Quantum Field Theory describing very successfully the electromagnetic as well as the weak and strong nuclear interactions.

- To finish our review of unification in physics, we note that in 1916 Einstein published his theory of General Relativity where he combined the idea of Special Relativity with Newtonian Gravity. The resulting theory provides a 
very accurate description of the gravitational interaction and so again, the unification of different physical concepts has led to a better understanding of nature.

\section{Towards a "Theory of Everything"}

In view of figure 1.1, there is one final step to be done, namely the unification of Quantum Field Theory, in particular the Standard Model of Particle Physics, and General Relativity to a so-called "Theory of Everything". As suggested by the previous examples, such a step is expected to provide us with a deeper understanding of the original theories as well as to give us novel insight into physics.

But let us be more precise about why we are interested in a better understanding of General Relativity and Quantum Field Theory, hopefully in terms of a unified theory.

- General Relativity is a classical theory valid at very large distances. However, solutions to this theory, in particular black hole solutions, generically contain singularities which signal an inconsistency at very small scales. Thus, a theory of Quantum Gravity resolving these singularities is necessary.

- Quantum Field Theories on the other hand feature infinities at many instances. Nevertheless, it is possible to extract sensible information from certain Quantum Field Theories after regularisation and renormalisation. Although this formalism is extremely successful, however, it does not provide us with a satisfactory understanding of Quantum Field Theory.

- In certain situations, nature is well-described neither in terms of a Quantum Field Theory nor General Relativity but demands a unified description. Examples for such situations are the early universe after the big bang, and phenomena at non-negligible curvature in the vicinity of black holes.

Coming back to figure 1.1, although the final step to a unified theory of quantum physics and gravity is highly desirable, it is an extremely difficult task. One of the reasons is that Quantum Field Theory and General Relativity are very different in nature. In particular, the former describes physics on very small scales in terms of probabilities while the latter is a classical and deterministic theory important only at very large scales. As one can imagine, a framework incorporating these two concepts is rather difficult to develop.

But there are approaches towards a unification of Quantum Field Theory and General Relativity. For example, a natural attempt is to quantise gravity as a Quantum Field Theory of metric fluctuations around a fixed background. However, usually 
this leads to ultra-violet divergencies ${ }^{1}$ and it turns out that the resulting theory is non-renormalisable. Thus, such a formulation is not a fundamental theory. More promising candidates are Loop-Quantum Gravity being a quantum theory of space-time, and String Theory to which we turn now.

\section{Bosonic String Theory}

After having motivated why unifying Quantum Field Theory an General Relativity is a worthwhile task, we will now explain why String Theory is a promising candidate for such a unified theory.

We begin by stating the action for the theory of the so-called bosonic string. Consider a two-dimensional world-sheet $\Sigma$ parametrised by coordinates $\sigma^{\alpha}=\left(\sigma^{1}, \sigma^{2}\right)$ where $\sigma^{1}$ denotes the world-sheet space coordinate and $\sigma^{2} \in \mathbb{R}$ is world-sheet time. The metric on $\Sigma$ we call $h_{\alpha \beta}$. Next, we introduce bosonic fields $X^{\mu}\left(\sigma^{1}, \sigma^{2}\right)$ on this world-sheet with $\mu=0, \ldots, d-1$. These fields are maps from $\Sigma$ into a $d$-dimensional target space whose metric we denote by $g_{\mu \nu}$. Furthermore, the $X^{\mu}$ can be interpreted as the position of a string in this space which is illustrated in figure 1.2. Choosing then $g_{\mu \nu}=\eta_{\mu \nu}$ with $\eta_{\mu \nu}$ the flat Minkowski metric, the so-called Polyakov action reads

$$
\mathcal{S}=-\frac{T}{2} \int_{\Sigma} d^{2} \sigma \sqrt{-\operatorname{det} h} h^{\alpha \beta} \partial_{\alpha} X^{\mu} \partial_{\beta} X^{\nu} \eta_{\mu \nu}
$$

where $T=\left(2 \pi \alpha^{\prime}\right)^{-1}$ stands for the tension of the string and $\alpha^{\prime}$ is related to the fundamental length scale of a string as $l_{s}=2 \pi \sqrt{\alpha^{\prime}}$. Finally, we observe that for $\Sigma$ being an infinite cylinder the action describes a closed string, while for $\Sigma$ being an infinite strip (1.1) describes an open string.

The action (1.1) can be taken as the starting point for the study of bosonic String Theory and we will now highlight some important features thereof. (For a more detailed introduction to String Theory, we ask the reader to consult the standard textbooks $[7-14]$.)

- Upon quantising the theory given by (1.1), the fields $X^{\mu}$ are promoted to operators. The corresponding Fourier modes are interpreted as vibration modes of the string and carry quantum numbers of the $d$-dimensional Poincaré group. They can thus be interpreted as particles in the target space.

\footnotetext{
${ }^{1}$ A possible exception is so-called $\mathcal{N}=8$ supergravity which is expected to be finite in the ultra-violet [4-6].
} 


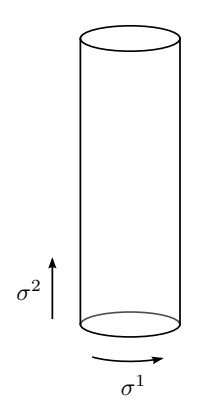

two-dimensional world-sheet $\Sigma$

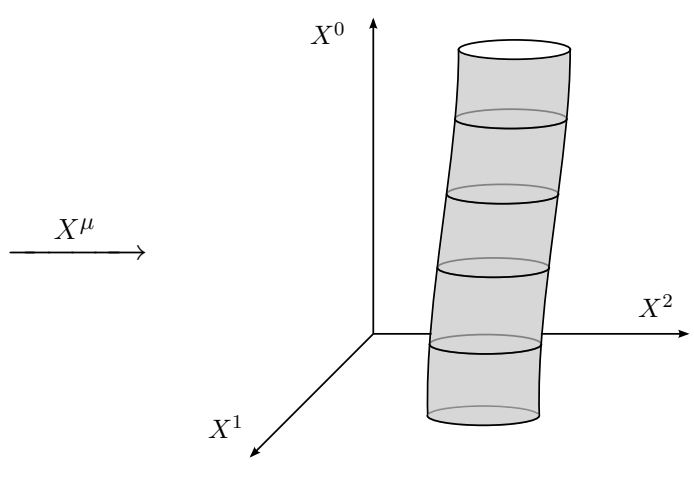

$d$-dimensional target space

Figure 1.2: Illustration of a two-dimensional closed string world-sheet $\Sigma$ mapped via $X^{\mu}$ into a $d$-dimensional target space the string is moving in.

Therefore, particles in String Theory arise as different excitations of a string and from this point of view, String Theory is a unified theory since all particles have the same origin.

- In the spectrum of closed strings, one always finds a massless spin two particle. Further analysis then shows that the latter can be identified with the graviton which is the gauge field of the gravitational interaction. Therefore, String Theory naturally contains gravity.

- As one can verify, at the classical level the action (1.1) is invariant under Weyl rescalings $h_{\alpha \beta}(\sigma) \rightarrow e^{2 \Lambda(\sigma)} h_{\alpha \beta}(\sigma)$. At the quantum level, however, this symmetry in general is anomalous. Requiring the Weyl symmetry to also be a symmetry of the quantum theory leads to a constraint on the dimension of the target space-time. In particular, the bosonic string has vanishing Weyl anomaly only in $d=26$ space-time dimensions. ${ }^{2}$

Of course, we experience four rather than 26 space-time dimensions. But let us note that this situation is changed to $d=10$ for the superstring to be considered below. More importantly, we would like to highlight the remarkable feature of String Theory to require a specific number of space-time dimensions for its consistency.

\footnotetext{
${ }^{2}$ Two comments are in order. First, requiring the theory to be unitary also leads to $d=26$ for the bosonic string. Second, the vanishing of the Weyl anomaly only gives a constraint on the total central charge of the Conformal Field Theory (CFT) defined by (1.1). Partially replacing the free boson CFT by a more abstract theory with the correct central charges also gives a consistent String Theory. However, in this case the geometric interpretation of the target space is usually lost. Examples for such constructions are the Gepner models.
} 
- Upon generalising the Polyakov action (1.1) to curved backgrounds via $\eta_{\mu \nu} \rightarrow$ $g_{\mu \nu}$ and including other background fields, the vanishing of the Weyl anomaly leads to Einstein's equation for the target space metric. This shows that String Theory incorporates General Relativity. However, there are corrections to Einstein's equation related to the string length encoded in $\alpha^{\prime}$, which become important at small scales.

- In String Theory, the fundamental objects are not point-like but have a finite size typically implying the absence of ultra-violet divergencies in the theory. Therefore, String Theory is expected to provide a consistent theory of Quantum Gravity.

Furthermore, due to their finite size, strings are not able to probe space-time up to arbitrary small scales because the resolution in scattering experiments is given by the size of the probes. Therefore, in some sense, space-time in String Theory is discretised and from this point of view, singularities of space-time are avoided.

In conclusion, we have motivated why bosonic String Theory is a promising starting point for a unified theory of Quantum Field Theory, respectively Particle Physics, and General Relativity.

\section{Superstring Theory}

However, the bosonic string exhibits a number of problems which demand for further development. In particular, bosonic String Theory contains a tachyon in the spectrum and thus the ground state is unstable. In addition, the spectrum does not contain objects which transform as spinors of the 26-dimensional Lorentz group implying the absence of fermionic degrees of freedom in the target space.

To remedy these shortcomings, one considers Superstring Theory, which is a supersymmetric extension of the bosonic string including fermionic degrees of freedom on the world-sheet $[15,16] .{ }^{3}$ This leads to a space-time supersymmetric theory with fermionic fields in the target space [17-21] and, as we mentioned previously, the vanishing of the Weyl anomaly now fixes the space-time dimension to be $d=10$.

However, there is not only one Superstring Theory but several constructions are possible. Well below the characteristic energy scale of a string, $M_{s}=\left(\alpha^{\prime}\right)^{-\frac{1}{2}}$, these Superstring Theories are described by supergravity theories and can be characterised by the latter. Although the supergravity theories capture only the massless

\footnotetext{
${ }^{3}$ Supersymmetry is a symmetry which relates bosonic and fermionic degrees of freedom. That is, for every boson in the theory there exists a fermion, its so-called superpartner, and vice versa. Also possible are extended supersymmetries with more than one superpartner for each field.
} 
excitations of the string and genuine string effects important at energy scales $M_{s}$ are neglected, one can still learn much about Superstring Theory.

As it turns out, there are five stable and consistent Superstring Theories leading to a supersymmetric theory in the target space. We briefly list these possibilities [11].

Type I String Theory is a theory of open and closed unoriented superstrings in a ten-dimensional target space-time. Its low energy effective description is an $\mathcal{N}=1$ Super Yang-Mills theory with gauge group $S O(32)$ coupled to type I supergravity.

Type IIA and type IIB String Theories are theories of closed oriented superstrings in ten dimensions. Their low energy effective descriptions are type IIA respectively type IIB supergravity in $d=10$.

Heterotic String Theory is a combination of the bosonic string in the leftmoving and the superstring in the right-moving sector of a closed string. Space-time is ten-dimensional and the allowed gauge groups are $\operatorname{Spin}(32) / \mathbb{Z}_{2}$ as well as $E_{8} \times E_{8}$. The low energy theories are $\mathcal{N}=1$ Super Yang-Mills theories coupled to type I supergravity.

Furthermore, there are two non-supersymmetric and unstable theories, type 0A and type $0 \mathrm{~B}$ - and there exist two theories related to String Theory which are however no String Theories in the strict sense.

M-Theory is the strong coupling limit of type IIA Superstring Theory with eleven-dimensional Poincaré invariance.

F-Theory is a geometric description of type IIB Superstring Theory which is formulated in a formal twelve-dimensional space-time (compactified on a torus) [22].

The remarkable observation made in the mid-nineties of the last century is that all these formulations of Superstring Theory are related via various dualities. The known Superstring Theories can therefore be regarded as different limits of a more fundamental, possibly unique theory yet to be fully discovered.

\section{Open Strings and D-Branes}

When discussing the world-sheet action (1.1) for the bosonic string, we already mentioned that a world-sheet $\Sigma$ with the topology of an infinite strip describes an open string. In the target space, the endpoints of the open string are confined to hypersurfaces which are called D-branes (see figure 1.3) and, as it turns out, in addition to strings also D-branes are fundamental objects of the theory. 


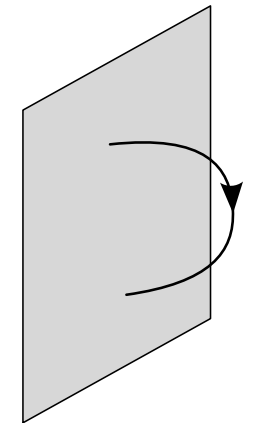

(a) Open string with both endpoints on the same D-brane

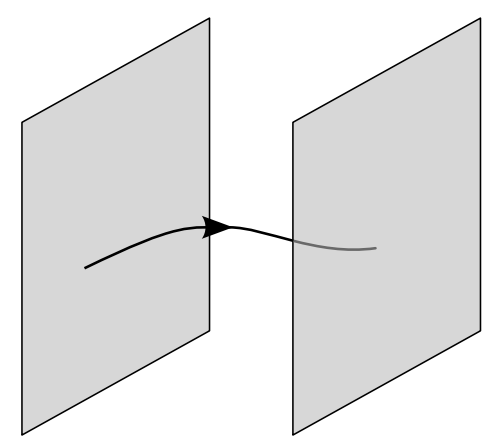

(b) Open string stretched between different D-branes

Figure 1.3: Illustration of open strings and D-branes.

Let us remark that similar to the reflection of ordinary waves, the endpoints of an open string lead to an identification of the left- and right-moving sector of the string. Therefore, D-branes do not exist for the heterotic String Theories due to their left-right asymmetry. Since we are interested in open strings in the following, we are going to focus on type I respectively type II String Theories.

Let us now collect some properties of open strings and D-branes. A summary of this discussion can be found in figure 1.4 .

- The dynamics of the massless degrees of freedom originating from open strings with both endpoints on the same brane is described by a pure Super YangMills Theory living on the world-volume of the D-brane. For a stack of $N$ coincident D-branes, the corresponding gauge group is $U(N) .^{4}$ Therefore, gauge theories in String Theory have a geometric origin.

- There are not only open strings with both endpoints on the same stack of D-branes, but open strings can also stretch between different stacks. As it turns out, in the case of intersecting branes with open strings localised at the intersection locus, it is possible to obtain chiral matter.

More concretely, for two intersecting stacks of $N_{1}$ and $N_{2}$ D-branes, so-called chiral matter transforming in bi-fundamental representations of the gauge group $U\left(N_{1}\right) \times U\left(N_{2}\right)$ is counted by a certain topological invariant. We will be more precise about this point in section 4.2 .

- In general, D-branes are allowed to intersect multiple times. Each (topologically invariant) intersection will give rise to one copy of chiral matter

\footnotetext{
${ }^{4}$ When considering an orientifold projection to be explained below, also gauge groups $S O(2 N)$ and $S p(2 N)$ are possible. Furthermore, in F-theory more general gauge groups are allowed.
} 


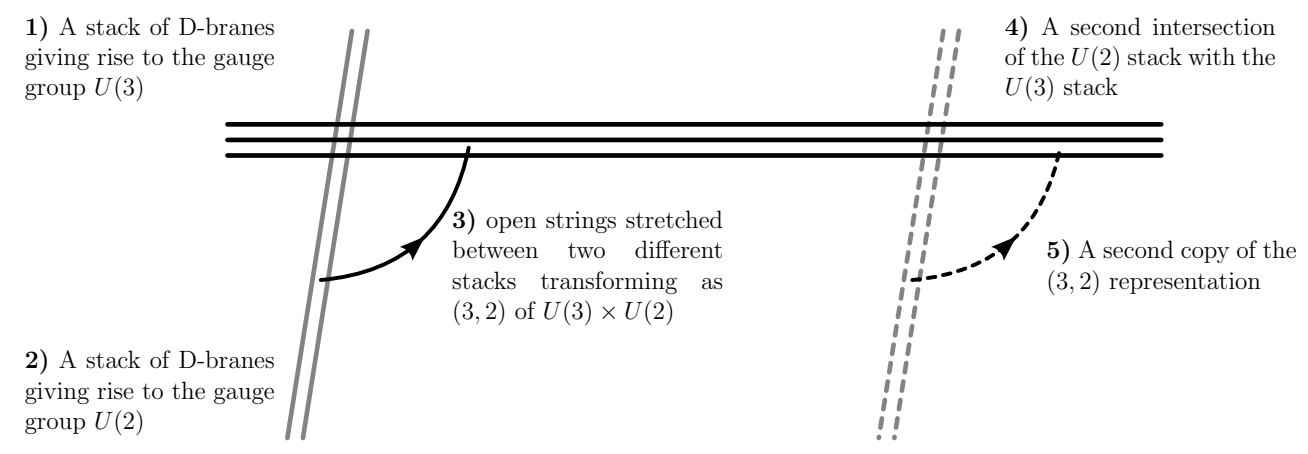

Figure 1.4: Illustration of intersecting branes, in particular, of the geometric interpretation of gauge groups, bi-fundamental representations and family replication.

transforming in bi-fundamental representations which goes under the name of family replication.

To summarise, gauge theories in String Theory can be realised by open strings whose endpoints are confined to D-branes. The latter provide a geometric understanding of the origin of gauge groups, chiral matter and family replication.

We furthermore mention that open strings can join to form closed strings and so a theory of open strings necessarily comes with the closed string sector containing the graviton. Therefore, in String Theory gauge theories and the gravitational interaction are closely tied together.

\section{Some Concluding Remarks}

At this point, we hope that we could convince the reader of the advantages of a unified theory of Quantum Field Theory and General Relativity, and why String Theory is a promising candidate for such a "Theory of Everything". To finish this section, let us briefly highlight some achievements and open problems of String Theory.

Over the years, String Theory has advanced to a very complex and rich theory with a variety of subfields and directions of specialisation. We will comment on some of them.

- For instance, people study Topological String Theory and String Field Theory with the aim of a better understanding of the theory on a formal and fundamental level. Related to this point are mathematical questions in the realm of Conformal Field Theory and Algebraic Geometry. 
The results obtained in this branch of String Theory have influenced several other fields of research. For instance, the discovery of mirror symmetry in String Theory became of interest for mathematicians who further developed this idea. Similarly, people studying Conformal Field Theory have benefited much from the interaction with String Theorists.

We also mention the remarkable observation that by counting microstates in String Theory, the classical thermodynamical entropy of BPS black-holes can be reproduced [23].

- Another important field of research in String Theory is related to the AdS/CFT correspondence [24,25]. This correspondence is a conjecture about the duality between String Theory on Anti-de Sitter spaces (AdS) and certain Conformal Field Theories (CFTs). As such, it may provide a way for an understanding of Quantum Gravity.

In addition, the AdS/CFT correspondence provides a tool to study strongly coupled Field Theories or certain condensed matter systems in terms of String Theory. In this way, for instance, it may help to gain a better analytic control over the QCD sector of the Standard Model.

- Another area of research within String Theory is concerned with the connection between String Theory and Particle Physics, and goes under the name of String Phenomenology.

Ideas originating from this branch have influenced for instance people interested in Physics Beyond the Standard Model where the concepts of (large) extra dimensions [26] or brane worlds [27-29] are now accepted scenarios.

However, although there has been and there is progress in the understanding of String Theory, some questions are still without a satisfying answer. We will briefly comment on some of these.

- As we have emphasised, String Theory incorporates General Relativity. But in its present form, gravity is described as fluctuations of the graviton around a constant background and thus only provides a perturbative description of the gravitational interaction.

- The five Superstring Theories are mostly studied in a perturbative regime. However, it is surely needed to understand these theories also in the nonperturbative regime, which hopefully leads to a better understanding of the underlying formulation of String Theory.

- Even though there are only five Superstring Theories, they allow for a great number of solutions. It is hard to estimate this number but let us quote the famous results $10^{1500}$ and $10^{500}$ which appeared in [30] and [31], respectively. 
It is not a conceptual problem of String Theory to have a huge number of solutions. In the case of Quantum Field Theories, for instance, one can construct an infinite number of theories - on the other hand, in String Theory the number of solutions is expected to be finite [32].

One of the main challenges in String Phenomenology is to find a solution of String Theory which describes our world. Presently, however, the theory does not predict or single out a specific vacuum. This is no conceptual problem of String Theory - similarly, it is no conceptual shortcoming of Quantum Field Theory to not predict the Standard Model, nor of General Relativity not to predict the precise form of our solar system.

- We finally remark on the falsifiability of String Theory. In principle, String Theory is a falsifiable theory because if we perform an experiment at the string scale, we can test whether String Theory is correct. It is again not a conceptual but only a practical shortcoming not being able to do such experiments..$^{5}$

\subsection{String Phenomenology}

As we have mentioned in the previous section, String Phenomenology is a branch of research in String Theory which is concerned with establishing a connection between String Theory and Particle Physics.

String Theory

in $d=10$
Particle Physics in $\mathrm{d}=4$

In the present section, we are now going to be more precise about what we mean with "Particle Physics" and we will explain how this connection can be achieved. (For recent reviews on this subject, see for instance [34,35].)

\section{What Do We Mean With "Particle Physics"?}

A very successful theory describing the electromagnetic as well as the weak and strong nuclear interactions of our world is the so-called Standard Model of Particle Physics. This theory has been tested by various means up to a high accuracy and

\footnotetext{
${ }^{5}$ Two comments are in order. First, in our universe energies of the order of the string scale may be reached and thus astrophysical data might provide a possibility to test String Theory. Second, if the string scale is close to the electroweak scale then indeed the LHC could measure String Theory effects. (For a recent and very specific analysis of this point see for instance [33].)
} 
the only missing point is the discovery of the Higgs boson to hopefully be made by the LHC.

However, despite its great success, the Standard Model is not regarded as a fundamental theory but rather as an effective one. Some of the reasons for this point of view are the following.

- The Standard Model does not include gravity and thus is no fundamental theory. It is an effective theory valid when the gravitational interaction can be neglected.

- There is no explanation why space-time should be four-dimensional. Of course, we experience only four space-time dimensions but a fundamental theory should address this point.

- The Standard Model contains more than 20 free parameters which have to be measured. A fundamental theory is expected to have much less arbitrary parameters. Furthermore, the origin of the Standard Model gauge group $S U(3) \times S U(2) \times U(1)$ as well as that of the three families of quarks and leptons is not clear.

- Another issue in the Standard Model is the so-called hierarchy problem. More concretely, if the Standard Model is valid up to an energy scale $\Lambda$ then quantum corrections to the mass of the Higgs boson turn out to be of order $\Lambda^{2}$. But for the Higgs mechanism to generate the correct masses for other Standard Model particles, the renormalised Higgs mass should be of the order of a few hundred $\mathrm{GeV}$.

Therefore, the bare Higgs mass has to be fine-tuned to a high degree such that the quantum corrections of order $\Lambda^{2}$ lead to a Higgs mass of the right magnitude. Furthermore, it is not clear why the electroweak scale respectively the Higgs mass is so much smaller than the natural cutoff scale of the theory being for instance the Planck scale.

- It is not clear why the electroweak sector of the Standard Model violates the CP symmetry, and why the sector of strong interactions does not seem to do so. In particular, the $\mathrm{CP}$ violating term in the QCD Lagrangian has to have a prefactor which is unnaturally small.

- The Standard Model does not make appropriate contact with cosmology. In particular, there is no candidate for dark matter nor is there an explanation for dark energy respectively the small positive cosmological constant.

Let us emphasise that the items listed here do not disqualify the Standard Model as a very successful effective theory. These points rather indicate that it is not a fundamental description of nature. 
There are a number of approaches to overcome some of the issues mentioned above. We will list some of them.

- In the so-called Technicolor Theories [36,37], the Higgs field responsible for electroweak symmetry breaking is replaced by fermion condensates. In this way, the hierarchy problem is avoided. However, some of the simpler technicolor models are not in agreement with experiment.

- A second idea to improve the Standard Model is to introduce a new symmetry between bosons and fermions, supersymmetry, which led to the development of the so-called Minimal Supersymmetric Standard Model (MSSM) to be discussed below.

- A third approach is the following. When running the gauge couplings of the Standard Model to higher energies, they pairwise meet in three different points. However, when considering the MSSM instead of the Standard Model, all three gauge couplings unify at an energy of around $1.2 \times 10^{16} \mathrm{GeV}$ suggesting a so-called Grand Unified Theory (GUT). In this approach, the Standard Model gauge group is a subgroup of the GUT group, for instance $S U(5)$ [38] or $S O(10)$ [39]. Furthermore, GUTs predict relations among masses and gauge couplings thereby reducing the number of arbitrary parameters.

However, in GUTs one assumes that from the weak to the GUT scale, these are 14 orders of magnitude, no new physics changing the running of couplings occurs. Also, Grand Unified Theories generically predict proton decay which is not observed by experiment.

Let us now be more precise about the Minimal Supersymmetric Standard Model as it will be important for our following discussion. We list some interesting features of this theory but also point out some of its problems.

- The MSSM provides a solution to the hierarchy respectively fine-tuning problem since in contrast to the Standard Model, each particle has a superpartner whose contribution cancels the first order quantum corrections to the Higgs mass. As a consequence, the fine-tuning of the bare Higgs mass is avoided.

- In order to explain the stability of the proton, R-parity is introduced. This implies that the lightest supersymmetric particle is stable, and it turns out that it is a natural candidate for dark matter.

- At low energies, we do not observe the superpartners of ordinary matter. Therefore, supersymmetry has to be broken, preferably at a scale of a few $\mathrm{TeV}$, which may be verified by the LHC.

- However, despite some promising features, the MSSM suffers from certain drawbacks. For instance, the number of arbitrary parameters has been in- 
creased from around 20 in the Standard Model to over 120 for the MSSM.

Furthermore, the MSSM exhibits the so-called $\mu$ problem described in the following. The mass parameter of the Higgs doublets, usually denoted by $\mu$, is crucial for giving vacuum expectation values to the Higgs fields which in turn give masses to the matter fields. Therefore, $\mu$ should be of the order of the electroweak scale which is several orders of magnitude smaller than the natural cutoff scale being for instance be the Planck scale.

To conclude, the MSSM is a promising extension of the well-tested Standard Model of Particle Physics addressing some of the problems of the latter. However, the MSSM still is not a fundamental description of nature.

\section{The Task of String Phenomenology}

In the previous paragraph, we have illustrated that the Standard Model of Particle Physics is a very successful theory describing our world at low energies, but also that it is only an effective description. In order to solve some of its problems, we concentrated on the Minimal Supersymmetric Standard Model and explained some of its features. Following this approach, we can refine the illustration at the beginning of this section as follows. ${ }^{6}$

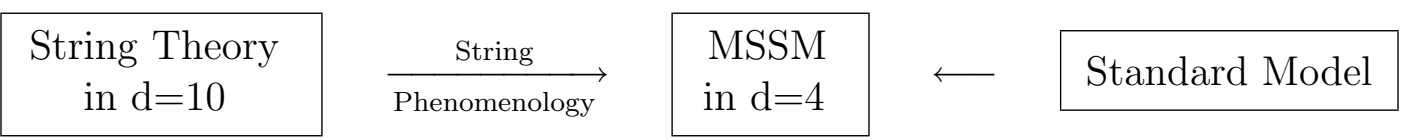

In order to make contact between String Theory defined in a ten-dimensional space-time and the MSSM in four dimensions, two basic problems have to be addressed. One is to go from ten to four dimensions which is usually achieved via compactification, and the other is to engineer a low energy theory resembling the MSSM or some extension thereof.7

Finally, although our forthcoming discussion in this work is applicable to all five Superstring Theories, let us make clear that

In the following, we focus on type II Superstring Theories.

\footnotetext{
${ }^{6}$ This is of course an oversimplified picture expressing the personal viewpoint of the author. There are also attempts to construct the Standard Model, or some other extension thereof, directly. However, in recent years people have focused on connecting String Theory to the MSSM or some GUT extension. This may change when the LHC provides new experimental data.

${ }^{7}$ Note that String Theory seems to have many solutions which exhibit properties of the MSSM (see for instance [40-42]). However, a more detailed analysis of these models together with a better understanding of String Theory may change this picture.
} 


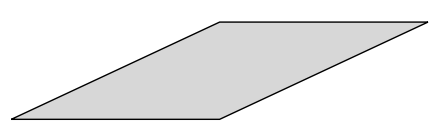

(a)

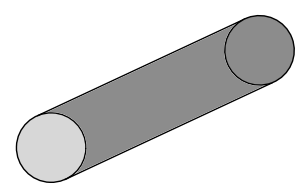

(b)

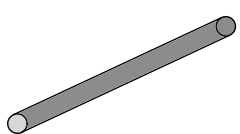

(c)

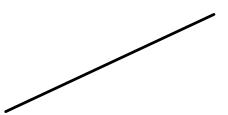

(d)

Figure 1.5: Illustration of a compactification from two dimensions to one dimension. In (a) one can see two uncompactified directions, in (b) one direction is rolledup and in (c) the radius of the circle is decreased. When as in (d) the radius is small enough, the cylinder appears as a line.

\section{Compactification and Moduli Stabilisation}

Let us continue by addressing the question of how to go from ten dimensions to a four-dimensional space-time. Usually, this is achieved by compactifying six spatial dimensions and making them very small such that they have escaped our detection so far. An illustration of this idea can be found in figures 1.5 .8

Next, we observe that there are many different ways to compactify six flat dimensions. To illustrate this point, let us consider two dimensions where, among others, we can choose the sphere, the torus and orbifolds thereof. In higher dimensions things are much more complicated as there are many more possibilities to choose a compact space. We note that this situation precisely corresponds to one of the problems in String Theory: in ten dimensions there are only five Superstring Theories which, however, exhibit an enormous number of four-dimensional vacua.

Nevertheless, not every six-dimensional compactification manifold, henceforth also called internal space, is allowed. In order to obtain the right amount of supersymmetry in four-dimensions, this manifold has to be a so-called Calabi-Yau threefold. 9

Let us now turn to the low energy effective action describing String Theory compactifications on Calabi-Yau manifolds. In particular, the size and the shape of the compactification space are encoded in its metric. Since in supergravity theories the

\footnotetext{
${ }^{8}$ Let us mention that there are alternative ideas to deal with a higher-dimensional spacetime. For instance, in the DGP scenario [29] our world is realised on a brane embedded in a five-dimensional space and gravity is modified on short distances to be concentrated on the brane. Another approach, as mentioned before, is to replace the six-dimensional free boson CFT by a more abstract Conformal Field Theory which however makes the geometric interpretation ambiguous.

${ }^{9}$ An $n$-dimensional Calabi-Yau manifold is a complex Kähler manifold with vanishing first Chern class, or equivalently, with $S U(n)$ holonomy.
} 
metric is a dynamical field, upon compactification the parameters corresponding the size and shape are promoted to fields in the four-dimensional theory. More concretely, the size of the internal manifold is encoded in the so-called Kähler moduli while the shape is encoded in the so-called complex structure moduli. However, in the low energy effective theory, these a priori massless fields are unwanted for the following reasons.

- First, the presence of massless particles in the early universe can modify the abundances of hydrogen and helium during that time and thereby destroy the very successful predictions of Big Bang Nucleosynthesis [43]. Therefore, moduli have to acquire a mass.

- Second, many quantities of the low energy effective theory such as gauge and Yukawa couplings depend on the moduli but should be fixed. Therefore, moduli fields have to acquire a mass.

- Finally, moduli generically give rise to so-called fifth forces. The experimental bound on the masses of such fields is $m>10^{-4} \mathrm{eV}[44,45]$.

The process of giving a mass to the moduli and thereby fixing their value goes under the name of moduli stabilisation and is a central topic of this thesis.

As we will explain in the course of this work, for the purpose of moduli stabilisation not only a perturbative description is needed but also non-perturbative effects, invisible to perturbation theory, have to be taken into account. An example for the latter are instantons in Euclidean Yang-Mills theory which are (anti-)self dual finite action solutions of non-perturbative nature (see for instance [46] and references therein). In String Theory, such non-perturbative effects originate for instance from so-called D-brane instantons which we will study in some detail in chapters 5 and 8 .

\section{Model Building}

Another task to be performed when connecting String Theory to Particle Physics is to construct models which reproduce the MSSM or some extension thereof. Since we are focussing on type II String Theories, this is done by placing D-branes in the compact space such that their intersections give rise to the desired spectrum and properties in the four-dimensional theory.

However, engineering the MSSM is not easily achieved because there are a number of constraints to be satisfied. Some of these conditions are the following.

- The so-called tadpole cancellation conditions have to be satisfied. From a geometric point of view, these conditions resemble the fact that in a compact 
space the overall charge has to vanish. (We will be more precise about this interpretation in footnote 1 of chapter 2.)

- The tadpole cancellation conditions correspond to the cancellation of the topological charges of D-branes. However, D-branes are actually classified by Ktheory [47] which leads to additional constraints [48].

- Further restrictions arise from the supersymmetry conditions of D-branes which ensure their stability.

- We also require the low energy effective theory to be free of anomalies. In String Theory, chiral anomalies are taken care of by the Green-Schwarz mechanism [49] provided the tadpole cancellation conditions are satisfied. We will discuss this point in section 7.2 in detail. Furthermore, also the so-called Freed-Witten anomalies have to vanish [50] which we discuss in section 4.5.

In addition to the String Theory consistency conditions, a number of phenomenological constraints have to be satisfied in order for a model to describe our world. Let us list some of them.

- There are some obvious properties which have to be ensured such as the correct gauge group, the right number of generations of matter and the absence of exotic matter. Since for instance the rank of the total gauge group is constrained by the tadpole cancellation conditions, these features are usually not easily achieved.

- Next, the gauge and Yukawa couplings of the low energy theory have to be in a phenomenologically viable regime. However, since for example the gauge couplings are related to the size of the D-brane in the compactification space, this property depends on how moduli are stabilised.

- We finally note that supersymmetry of the low energy theory has to be broken in the region of a few $\mathrm{TeV}$ in order to be phenomenologically acceptable. As we will explain, this generically is in tension with the correct order of the gauge couplings of the D-branes. An interesting solution to this issue is the so-called LARGE Volume Scenario which will be discussed in chapter 6 .

To conclude, String Theory models have to satisfy a number of consistency conditions which make it non-trivial to construct a viable model of Particle Physics. 


\subsection{Overview}

We close this introductory chapter by providing an overview on the structure of this thesis. In particular, this work is organised in five parts containing the following topics.

Part I - Introduction to String Theory

Part II - Introduction at a technical level providing the necessary notation, developping the required background material and recalling known results from the literature

Part III - Presentation of the author's original work consisting of the following three topics which have been published in the papers [51-53]

- Model Building Constraints for Type IIB Orientifolds with O3- and O7-Planes

- D-Brane Instantons

- Moduli Stabilisation in the LARGE Volume Scenario

Part IV - Summary and Outlook

Part V - Appendices

Let us emphasise that this work is concerned with three somewhat different topics which are however linked in many instances (please see figure 1.6). A detailed study of these connections has led for instance to the discovery of an important constraint on moduli stabilisation in the presence of an MSSM sector which we are going to discuss in section 8.2 .

After this general overview, let us now give a more detailed outline of this thesis.

- In chapter 2, we introduce compactifications on orientifolds of Calabi-Yau three-folds and we summarise the effective action for the closed as well as for the open sector of type IIB String Theory.

In chapter 3, we present some basic features of $\mathcal{N}=1$ supergravity theories.

- In chapter 4, we discuss model building constraints in the context of type IIB String Theory with O3- and O7-planes. In particular, we summarise the rules for computing the chiral spectrum, we explain the generalised GreenSchwarz mechanism for the cancellation of chiral anomalies, we comment on 


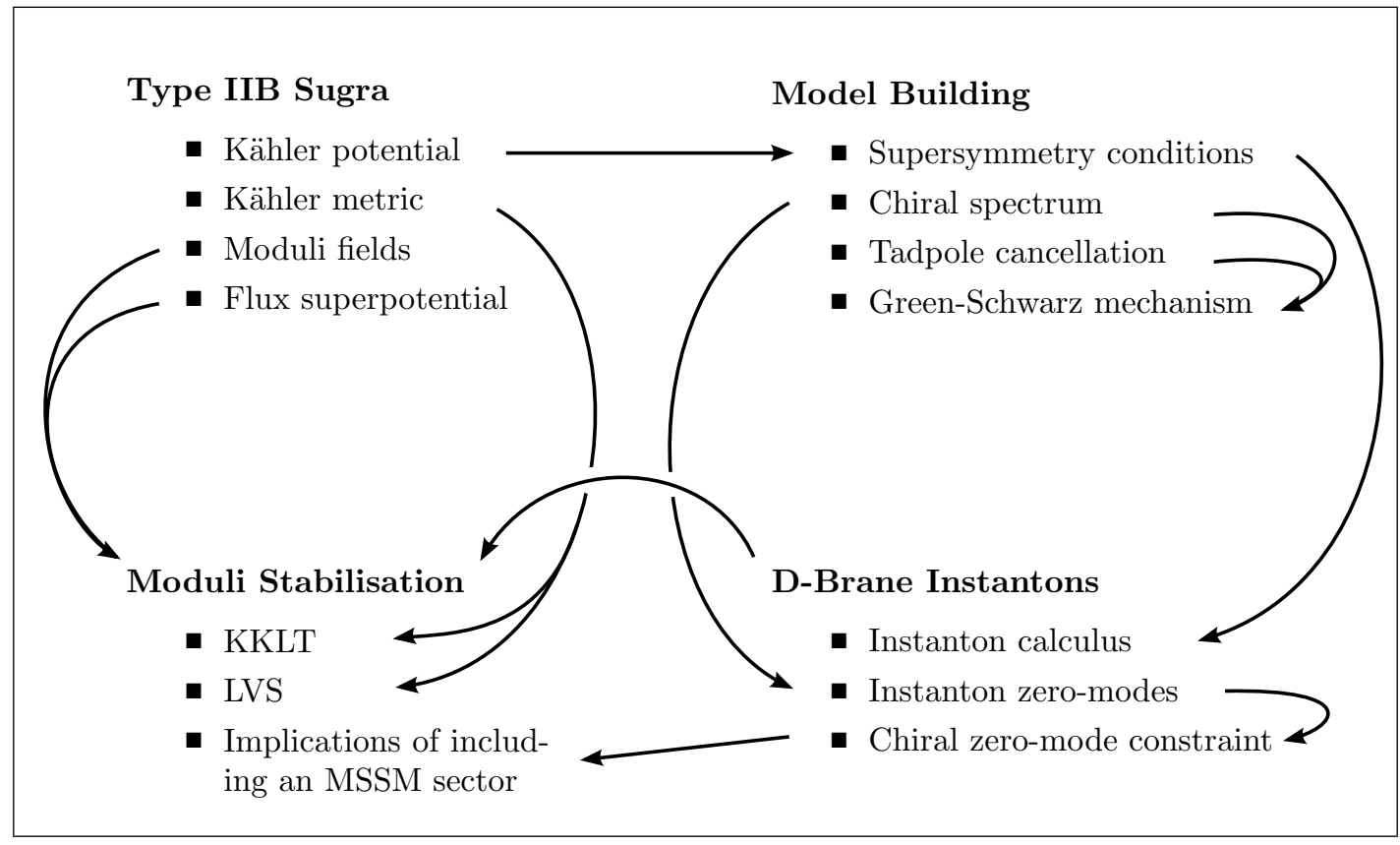

Figure 1.6: Overview on the interplay between the topics discussed in this work. The subjects mentioned here will be explained in due course, and we ask the reader to come back to this figure during his or her reading.

the supersymmetry conditions for D7-branes and we remark on the FreedWitten anomaly as well as on the so-called K-theory constraints.

Next, in chapter 5, we introduce so-called D-brane instantons and explain the instanton calculus developed in [54] as well as some generic features thereof.

In chapter 6, we discuss moduli stabilisation in the context of type IIB orientifolds with O3- and O7-planes. More concretely, we present the so-called KKLT scenario [55] and explain some of the problems of this approach. Then, we discuss in detail the so-called LARGE ${ }^{\mathrm{TM}}$ Volume Scenario ${ }^{10}[56,57]$ which is an advancement of the KKLT setup.

- In chapter 7, we derive the tadpole cancellation conditions for type IIB orientifolds with O3- and O7-planes, discuss in detail the generalised GreenSchwarz mechanism in this context and compute so-called Fayet-Iliopoulos terms. These results are based on the paper [53].

\footnotetext{
${ }^{10}$ The notation "LARGE" has been introduced by J. Conlon, F. Quevedo and collaborators in order to emphasise that the volume under consideration is not only large, but exponentially large.
} 
In chapter 8 we use the D-brane instanton calculus explained previously to derive the Affleck-Dine-Seiberg superpotential in String Theory, and we illustrate an important constraint for the stabilisation of moduli in the presence of an MSSM sector. This chapter is based on the papers [51] and [52].

Finally, in chapter 9 we reconsider moduli stabilisation in the context of type IIB String Theory with O3- and O7-planes being very careful about introducing an open string sector. In particular, we present a concrete example for a LARGE Volume Scenario where the constraint developed in chapter 8 has been taken into account. This discussion is based on the paper [52].

- In chapter 10, we summarise the results presented in this work and give an outlook for future directions of research. 


\section{Part II}

\section{Prerequisites}





\section{Chapter 2}

\section{Orientifold Compactifications and Effective Actions}

Let us begin the main part of this work by establishing the prerequisites and basic principles needed for the sequel. In particular, in this chapter we introduce compactifications of String Theory on orientifolds of Calabi-Yau manifolds, and we summarise the effective action for the closed as well as for the open sector of type IIB String Theory.

\subsection{Orientifold Compactifications}

\section{From Ten to Four Dimensions}

Throughout this work, we are going to focus on type IIB String Theory compactified from ten dimensions to four-dimensional Minkowski space on a real sixdimensional manifold $\mathcal{X}$

$$
\mathbb{R}^{9,1} \longrightarrow \mathbb{R}^{3,1} \times \mathcal{X}
$$

The most general ansatz for the metric which preserves four-dimensional Poincaré invariance, in a common convention, reads

$$
d s^{2}=e^{2 A(y)} \eta_{\mu \nu} d x^{\mu} d x^{\nu}+e^{-2 A(y)} g_{m n} d y^{m} d y^{n}
$$

where $x^{\mu}$ with $\mu=0, \ldots, 3$ are coordinates in $\mathbb{R}^{3,1}$ while $y^{m}$ with $m=1, \ldots, 6$ parametrise the compactification manifold $\mathcal{X}$. The factor $e^{2 A(y)}$ is called the warp factor which in String Theory can describe the back-reaction of fluxes on the 


$$
\begin{gathered}
h^{0,0} \\
h^{1,0} \quad h^{0,1} \\
h^{2,0} \quad h^{1,1} \quad h^{0,2} \\
h^{3,0} \quad h^{2,1} \quad h^{1,2} \quad h^{0,3} \\
h^{3,1} h^{2,2} h^{1,3} \\
h^{3,2} h^{2,3} \\
h^{3,3}
\end{gathered}
$$

(a) General complex three-manifold

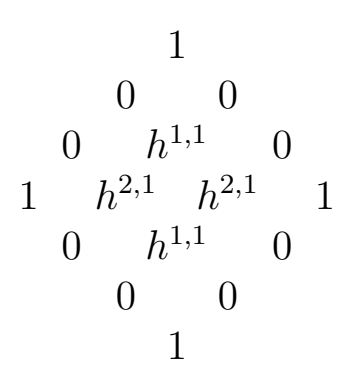

(b) Calabi-Yau three-fold

Figure 2.1: Hodge diamonds for a general and a specific complex three-manifold.

manifold $\mathcal{X}$ [58]. However, since we will be interested in volumes of internal space $\mathcal{X}$ which are large compared to the string scale, it turns out that the warp factor can be neglected and the metric $(2.2)$ factorises into two independent parts. This will be our assumption for the following.

The low energy effective theory of type II String Theories is usually formulated in terms of type II supergravities in ten dimensions which have 32 supercharges. In order to obtain $\mathcal{N}=2$ supersymmetry in four dimensions with eight supercharges, the compact space has to break some of the supersymmetries. In particular, $\mathcal{X}$ has to be a Calabi-Yau three-fold which is a complex Kähler manifold with vanishing first Chern class, or equivalently with exact $S U(3)$ holonomy.

Some of the topological data of a Calabi-Yau three-fold can be summarised in the so-called Hodge diamond shown in figures 2.1. (For more details see for instance [59].) There, $h^{p, q}$ are the dimensions of the Dolbeault co-homology groups $H_{\bar{\partial}}^{p, q}(\mathcal{X})$, and we see that for Calabi-Yau three-folds there are only two non-trivial numbers, namely $h^{2,1}$ and $h^{1,1}$. Furthermore, we note that a Calabi-Yau three-fold $\mathcal{X}$ is endowed with
a so-called Kähler form
$J \quad$ of degree $(1,1)$,
a unique holomorphic three-form
$\Omega_{3}$
of degree $(3,0)$.

\section{Orientifold Projection}

For our purpose, the situation described in the previous paragraph is not satisfactory yet because in view of String Phenomenology, we are not interested in $\mathcal{N}=2$ supersymmetry in $d=4$ but rather in the $\mathcal{N}=1$ case. In addition, eventually we want to introduce D-branes in order to engineer gauge theories. Since D-branes 
carry certain topological charges which have to be cancelled in a compact space, a source of negative charge with respect to the D-branes is needed. $1^{2}$

One therefore considers a so-called orientifold projection which in the present case takes the form $\Omega(-1)^{F_{L}} \sigma$ with $\Omega$ the world-sheet parity operator and $F_{L}$ the leftmoving space-time fermion number. (In equation 2.12 below, we will be more precise about these operators.) In the case of type IIB String Theory, $\sigma$ is a holomorphic involution on $\mathcal{X}$ and we choose its action on $J$ and $\Omega_{3}$ as

$$
\sigma^{*} J=+J, \quad \sigma^{*} \Omega_{3}=-\Omega_{3} .
$$

From the latter relation we see that $\sigma$ leaves invariant an even number of holomorphic coordinates and so the fixed loci of $\sigma$ on $\mathcal{X}$ can be points and complex two-dimensional submanifolds. In addition, $\sigma$ acts trivially on the four-dimensional part and thus leaves $\mathbb{R}^{3,1}$ invariant.

The fixed loci of the orientifold projection are called orientifold planes and usually carry negative topological charges with respect to the D-branes. In the present case, these are O3- and O7-planes where 3 respectively 7 denote the number of spatial dimensions of the orientifold plane. Note that it is always understood that O-planes occupy the time direction.

In passing, we mention that for type IIB String Theory also the action $\sigma^{*} \Omega_{3}=+\Omega_{3}$ is possible which gives rise to O9- and possibly to O5-planes. However, we do not consider this choice here.

\section{(Co-)Homology}

In order to prepare for our forthcoming discussion, we finish this section by establishing some notation on a more technical level. The holomorphic involution $\sigma$ introduced in the previous paragraph gives rise to a splitting of the co-homology groups $H_{\bar{\partial}}^{p, q}(\mathcal{X})$ into the even and odd eigenspaces of $\sigma^{*}$ (here we mainly follow [60])

$$
H_{\bar{\partial}}^{p, q}(\mathcal{X})=H_{\bar{\partial}+}^{p, q}(\mathcal{X}) \oplus H_{\bar{\partial}-}^{p, q}(\mathcal{X})
$$

\footnotetext{
${ }^{1}$ This is analogous to the following situation. Take a positron $e^{+}$carrying one unit of positive electric charge. In a non-compact space, the flux lines starting at $e^{+}$can escape to infinity and there is no conceptual problem. However, it is not possible to place a single positron in a compact space since the flux lines cannot escape to infinity and are not allowed to intersect. One has to introduce one unit of negative charge at which the flux lines can end, i.e. the overall charge in a compact space has to vanish.

${ }^{2}$ We do not introduce anti-branes which have opposite charge with respect to the D-branes but would break supersymmetry and could render a brane configuration unstable.
} 


\begin{tabular}{r||c||c} 
Basis for Co-Homology & Basis for Homology & Indices \\
\hline \hline$\left\{\omega_{I}\right\} \in H_{\bar{\partial}}^{1,1}(\mathcal{X})$ & $\left\{\Sigma^{I}\right\} \in H_{2}(\mathcal{X}, \mathbb{Z})$ & $I=1, \ldots, h^{1,1}$ \\
$\left\{\omega_{i}\right\} \in H_{\bar{\partial}+1}^{1,1}(\mathcal{X})$ & $\left\{\Sigma^{i}\right\} \in H_{2+}(\mathcal{X}, \mathbb{Z})$ & $i=1, \ldots, h_{+}^{1,1}$ \\
$\left\{\omega_{\hat{\imath}}\right\} \in H_{\bar{\partial}-}^{1,1}(\mathcal{X})$ & $\left\{\Sigma^{\hat{\imath}}\right\} \in H_{2-}(\mathcal{X}, \mathbb{Z})$ & $\hat{\imath}=1, \ldots, h_{-}^{1,1}$ \\
\hline$\left\{\sigma^{I}\right\} \in H_{\bar{\partial}}^{2,2}(\mathcal{X})$ & $\left\{\gamma_{I}\right\} \in H_{4}(\mathcal{X}, \mathbb{Z})$ & $I=1, \ldots, h^{1,1}$ \\
$\left\{\sigma^{i}\right\} \in H_{\bar{\partial}+}^{2,2}(\mathcal{X})$ & $\left\{\gamma_{i}\right\} \in H_{4+}(\mathcal{X}, \mathbb{Z})$ & $i=1, \ldots, h_{+}^{1,1}$ \\
$\left\{\sigma^{\hat{\imath}}\right\} \in H_{\bar{\partial}-}^{2,2}(\mathcal{X})$ & $\left\{\gamma_{\hat{\imath}}\right\} \in H_{4-}(\mathcal{X}, \mathbb{Z})$ & $\hat{\imath}=1, \ldots, h_{-}^{1,1}$ \\
\hline$\left\{\left[\alpha_{\kappa}\right],\left[\beta^{\lambda}\right]\right\} \in H_{+}^{3}(\mathcal{X}, \mathbb{R})$ & $\left\{\alpha_{\kappa}, \beta^{\lambda}\right\} \in H_{3+}(\mathcal{X}, \mathbb{Z})$ & $\kappa, \lambda=1, \ldots, h_{+}^{2,1}$ \\
$\left\{\left[\alpha_{\hat{\kappa}}\right],\left[\beta^{\hat{\lambda}}\right]\right\} \in H_{-}^{3}(\mathcal{X}, \mathbb{R})$ & $\left\{\alpha_{\hat{\kappa}}, \beta^{\hat{\lambda}}\right\} \in H_{3-}(\mathcal{X}, \mathbb{Z})$ & $\hat{\kappa}, \hat{\lambda}=0, \ldots, h_{-}^{2,1}$
\end{tabular}

Table 2.1: Summary of our notation for basis of (co-)homology groups.

The dimensions of these spaces will be denoted by $h_{ \pm}^{p, q}$ for which the following relations can be determined [60]

$$
\begin{array}{lll}
h_{ \pm}^{1,1}=h_{ \pm}^{2,2}, & h_{+}^{3,0}=h_{+}^{0,3}=0, & h_{+}^{0,0}=h_{+}^{3,3}=1 \\
h_{ \pm}^{2,1}=h_{ \pm}^{1,2}, & h_{-}^{3,0}=h_{-}^{0,3}=1, & h_{-}^{0,0}=h_{-}^{3,3}=0 .
\end{array}
$$

Next, we introduce a basis for each of the co-homology and corresponding homology groups which we have summarised in table 2.1. Note in particular, we use $i$ and $\kappa$ for the $\sigma$-even groups and $\hat{\imath}$ respectively $\hat{\kappa}$ for the $\sigma$-odd parts. Furthermore, the index $I=1, \ldots, h^{1,1}$ runs over both the $\sigma$-even and $\sigma$-odd subspaces.

Let us now turn to the third (co-)homology group of $\mathcal{X}$. We choose our basis of $H_{3}(\mathcal{X}, \mathbb{Z})$ in such a way that the Poincaré duals $\{[\alpha \ldots],[\beta \cdots]\}$ satisfy

$$
\int_{\mathcal{X}}\left[\alpha_{\kappa}\right] \wedge\left[\beta^{\lambda}\right]=l_{s}^{6} \delta_{\kappa}^{\lambda}, \quad \int_{\mathcal{X}}\left[\alpha_{\hat{\kappa}}\right] \wedge\left[\beta^{\hat{\lambda}}\right]=l_{s}^{6} \delta_{\hat{\kappa}}^{\hat{\lambda}},
$$

and all other combinations vanishing. Note that our conventions are such that each $p$-form has dimension $l_{s}^{p}$ where $l_{s}$ denotes the string length. For the even respectively odd $(1,1)$ - and $(2,2)$-co-homology groups, we choose basis such that the only non-trivial relations are of the following form

$$
\int_{\mathcal{X}} \omega_{i} \wedge \sigma^{j}=l_{s}^{6} \delta_{i}^{j}, \quad \quad \int_{\mathcal{X}} \omega_{\hat{\imath}} \wedge \sigma^{\hat{\jmath}}=l_{s}^{6} \delta_{\hat{\imath}}^{\hat{\jmath}} .
$$

Finally, the basis of four- and two-cycles shown in table 2.1 can be defined as the appropriate Poincaré duals of $\omega_{I}$ respectively $\sigma^{I}$, that is $\left[\gamma_{I}\right]=\omega_{I}$ and $\left[\Sigma^{I}\right]=\sigma^{I}$. 
More concretely, this means that

$$
\begin{aligned}
\int_{\gamma_{i}} \sigma^{j} & =l_{s}^{4} \delta_{i}^{j}, & \int_{\Sigma^{i}} \omega_{j} & =l_{s}^{2} \delta_{j}^{i}, \\
\int_{\gamma_{\hat{\imath}}} \sigma^{\hat{\jmath}} & =l_{s}^{4} \delta_{\hat{\imath}}^{\hat{\jmath}}, & \int_{\Sigma^{\hat{\imath}}} \omega_{\hat{\jmath}} & =l_{s}^{2} \delta_{\hat{\jmath}}^{\hat{\imath}} .
\end{aligned}
$$

\subsection{Effective Action for the Closed String Sector}

After having introduced an orientifold projection as well as our notation for the (co-)homology of the compactification manifold, we now turn to an effective field theory description of the closed sector of type IIB String Theory compactifications. In particular, we are going to explain how the dimensional reduction of type IIB supergravity on a Calabi-Yau three-fold is performed, summarise the resulting spectrum and comment on turning on background fluxes.

\section{Type IIB Supergravity Action}

As we have mentioned previously, the low energy limit of the closed sector of type IIB String Theory in ten dimensions can be formulated in terms of type IIB supergravity. The bosonic field content of this theory reads as follows. There is the metric $g$, the dilaton $\phi$, the Neveu Schwarz-Neveu Schwarz (NS-NS) two-form $B_{2}$ with field strength $H_{3}=d B_{2}$, and the Ramond-Ramond (R-R) $p$-form potentials $C_{p}$ with generalised field strengths

$$
\widetilde{F}_{p}=d C_{p-1}-H_{3} \wedge C_{p-3} .
$$

Note that in type IIB supergravity, the potentials $C_{p}$ appear for $p=0,2,4$ and the duality relation $\widetilde{F}_{5}=\star \widetilde{F}_{5}$ has to be imposed by hand. Here and in the following, the symbol $\star$ denotes the usual Hodge- $\star$-operator in ten dimensions (see for instance [59]).

However, in order to deal with D-branes, it turns out to be useful to employ the democratic formulation of type IIB supergravity [61] where the potentials $C_{p}$ for $p=6,8,10$ subject to the duality relations

$$
\widetilde{F}_{p}=(-1)^{\frac{p+3}{2}} \star \widetilde{F}_{10-p}
$$

are included as well. With $\left(2 \kappa_{10}^{2}\right)^{-1}=2 \pi l_{s}^{-8}$, where $l_{s}$ again denotes the string length, and $\mathrm{R}$ the ten-dimensional Ricci curvature scalar, the bosonic part of this 
type IIB (pseudo-)action reads [61]

$$
\begin{aligned}
\mathcal{S}_{\text {IIB }}=\frac{1}{2 \kappa_{10}^{2}} \int\left[e^{-2 \phi}(\mathrm{R} \star 1+4 d \phi \wedge \star d \phi\right. & \left.-\frac{1}{2} H_{3} \wedge \star H_{3}\right) \\
& \left.-\frac{1}{4} \sum_{p=1,3,5,7,9} \widetilde{F}_{p} \wedge \star \widetilde{F}_{p}\right] .
\end{aligned}
$$

\section{Orientifold Projection}

Next, we consider the orientifold projection. The action of $\Omega(-1)^{F_{L}}$ on the fields appearing in (2.11) is determined to be of the following form $[62,63,60]$

$$
\begin{array}{lll}
\Omega(-1)^{F_{L}} g=+g, & & \Omega(-1)^{F_{L}} B_{2}=-B_{2}, \\
\Omega(-1)^{F_{L}} \phi=+\phi, & & \Omega(-1)^{F_{L}} C_{p}=(-1)^{\frac{p}{2}} C_{p} .
\end{array}
$$

For later reference, let us now split the NS-NS two-form $B_{2}$ on $\mathcal{X}$ into parts which are even respectively odd under the holomorphic involution $\sigma$

$$
B_{2}^{(6)}=B_{2}^{+}+B_{2}^{-}, \quad \sigma^{*} B_{2}^{ \pm}= \pm B_{2}^{ \pm},
$$

where ${ }^{(6)}$ denotes the components of $B_{2}$ in the internal space $\mathcal{X}$. Taking into account the action of $\Omega(-1)^{F_{L}}$ given in $(2.12)$, we see that $B_{2}^{+}$is allowed to only take discrete values in order to be invariant under the full orientifold projection. On the other hand, $B_{2}^{-}$is invariant under $\Omega(-1)^{F_{L}} \sigma$ and remains as a continuous field in the low energy theory.

\section{Dimensional Reduction}

In order to go from the ten-dimensional to a four-dimensional effective action, we perform a Kaluza-Klein reduction on the compact manifold $\mathcal{X}$ and keep only the massless modes. That means, we expand the fields appearing in the action (2.11) in terms of the co-homology introduced in table 2.1 and evaluate the integration over $\mathcal{X} \cdot 3^{3}$ This will lead to to an $\mathcal{N}=2$ supersymmetric theory in four-dimensions,

\footnotetext{
${ }^{3}$ Let us illustrate this procedure for a massless ten-dimensional scalar field $\Phi$. Employing the notation given in $(2.2), \Phi(x, y)$ satisfies $\square_{10} \Phi(x, y)=0$ and the ten-dimensional d'Alembert operator splits into a four-dimensional part and the six-dimensional Laplace operator, i.e. $\square_{10}=\square_{4}+\Delta_{6}$. We then expand $\Phi(x, y)$ into eigenfunctions $\psi_{a}(y)$ of $\Delta_{6}$ as $\Phi(x, y)=\sum_{a} \phi_{a}(x) \psi_{a}(y)$. Denoting the (discrete) $\Delta_{6}$ eigenvalues of $\psi_{a}(y)$ by $\lambda_{a}$, the tendimensional Klein-Gordon equation from above becomes $\square_{4} \phi_{a}+\lambda_{a} \phi_{a}=0$ for each $a$ in which $\lambda_{a} \phi_{a}$ appears as a four-dimensional mass term. The $d=4$ massless modes correspond to harmonic functions satisfying $\Delta_{6} \psi_{a}=0$ which are in one-to-one correspondence with the cohomology of $\mathcal{X}$. The remaining modes have quantised masses proportional to the inverse size of $\mathcal{X}$, and for $\mathcal{X}$ sufficiently small, these can be integrated out.
} 


\begin{tabular}{|c|c|c|c|}
\hline Type of Multiplet & Multiplicity & Notation & Description \\
\hline gravity multiplet & 1 & $g_{\mu \nu}$ & $\begin{array}{l}\text { originating from the } d=4 \text { part of } \\
\text { the ten-dimensional metric }\end{array}$ \\
\hline vector multiplet & $h_{+}^{2,1}$ & $V^{\kappa}$ & $\begin{array}{l}\text { originating from the reduction of } \\
C_{4} \text { on } \sigma \text {-even three-cycles in } \mathcal{X}\end{array}$ \\
\hline chiral multiplet & $h_{-}^{2,1}$ & $U^{\hat{\kappa}}$ & $\begin{array}{l}\text { complex structure moduli encoded } \\
\text { in } \Omega_{3} \text { - originating from the } d=6 \\
\text { part of the ten-dimensional metric }\end{array}$ \\
\hline chiral multiplet & $h_{+}^{1,1}$ & $T_{i}$ & Kähler moduli - defined in 2.15 \\
\hline chiral multiplet & $h_{-}^{1,1}$ & $G^{\hat{\imath}}$ & $G$-moduli - defined in 2.16 \\
\hline chiral multiplet & 1 & $\tau=i S$ & axio-dilaton - defined in 2.14 \\
\hline
\end{tabular}

Table 2.2: Spectrum of the $\mathcal{N}=1$ supergravity theory originating from the dimensional reduction of the type IIB supergravity action on orientifolds of Calabi-Yau three-folds $[63,60,64]$.

which is broken to $\mathcal{N}=1$ via the orientifold projection. Since it is not a central part of this work, we do not perform this reduction explicitly but only summarise in table 2.2 the resulting spectrum. The detailed analysis can be found in [60,65]. The definition of the axio-dilaton $\tau=i S$, the moduli $G^{\hat{\imath}}$ and the Kähler moduli $T_{i}$ appearing in this table read

$$
\begin{aligned}
\tau & =C_{0}+i e^{-\phi}=i S, \\
T_{i} & =\frac{1}{l_{s}^{4}} \int_{\gamma_{i}}\left(\frac{1}{2} e^{-\phi} J \wedge J+i C_{4}+i B_{2}^{-} \wedge C_{2}+\frac{i}{2} \tau B_{2}^{-} \wedge B_{2}^{-}\right), \\
G^{\hat{\imath}} & =\frac{1}{l_{s}^{2}} \int_{\Sigma^{\hat{\imath}}}\left(C_{2}+\tau B_{2}^{-}\right),
\end{aligned}
$$

where $\left\{\gamma_{i}\right\} \in H_{4+}(\mathcal{X}, \mathbb{Z})$ and $\left\{\sum^{\hat{\imath}}\right\} \in H_{2-}(\mathcal{X}, \mathbb{Z})$ are the basis of four- respectively two-cycles introduced in table 2.1, and $B_{2}^{-}$had been introduce in (2.13).

Two comments are in order here. First, there are two common notations for the axio-dilaton: $\tau$ usually denotes the ten-dimensional complex field while the fourdimensional modulus is denoted by $S$. We will employ both notations, depending on the context. ${ }^{4}$ Second, in the presence of an open string sector with D-branes,

\footnotetext{
${ }^{4}$ Let us remark that there are also a number of different conventions used for the relation between the ten- and four-dimensional axio-dilaton. Also common is for instance $\tau=i \bar{S}[35]$. However, in the present work, the results have been made consistent with 2.14).
} 
the definition of the moduli above is modified in order to account for the additional structure $[66,67]$. This has however no effect for our following discussion.

\section{Background Fluxes}

Let us finally observe that we are allowed to turn on background fluxes in $\mathcal{X}$, that is, we can have non-vanishing VEVs for the field strengths [58]

$$
F_{3}=d C_{2} \quad \text { and } \quad H_{3}=d B_{2} .
$$

Because of the Dirac quantisation condition, the corresponding fluxes $\bar{F}_{3}$ and $\bar{H}_{3}$ are quantised. Furthermore, since we perform an orientifold projection $\Omega(-1)^{F_{L}} \sigma$, there are some subtleties due to the involution $\sigma$ on $\mathcal{X}[68,69]$. Although these issues can be dealt with, here we stay on firm grounds and impose the following quantisation conditions

$$
\begin{array}{ll}
\frac{1}{l_{s}^{2}} \int_{\alpha_{\hat{\kappa}}} F_{3}=2 f_{\hat{\kappa}} \in 2 \mathbb{Z}, & \frac{1}{l_{s}^{2}} \int_{\beta^{\hat{\lambda}}} F_{3}=2 f^{\hat{\lambda}} \in 2 \mathbb{Z}, \\
\frac{1}{l_{s}^{2}} \int_{\alpha_{\hat{\kappa}}} H_{3}=2 h_{\hat{\kappa}} \in 2 \mathbb{Z}, & \frac{1}{l_{s}^{2}} \int_{\beta^{\hat{\lambda}}} H_{3}=2 h^{\hat{\lambda}} \in 2 \mathbb{Z},
\end{array}
$$

with $\hat{\kappa}, \hat{\lambda}=0, \ldots, h_{-}^{2,1}$. Note that because $F_{3}$ and $H_{3}$ are odd under $\Omega(-1)^{F_{L}}$, we only turn on flux through cycles $\left\{\alpha_{\hat{\kappa}}, \beta^{\hat{\lambda}}\right\}$ odd under the orientifold projection. Using then (2.18) and (2.6), we can express the fluxes $\bar{F}_{3}$ and $\bar{H}_{3}$ in the following way

$$
\bar{F}_{3}=\frac{2}{l_{s}}\left(f^{\hat{\lambda}}\left[\alpha_{\hat{\lambda}}\right]-f_{\hat{\kappa}}\left[\beta^{\hat{\kappa}}\right]\right), \quad \bar{H}_{3}=\frac{2}{l_{s}}\left(h^{\hat{\lambda}}\left[\alpha_{\hat{\lambda}}\right]-h_{\hat{\kappa}}\left[\beta^{\hat{\kappa}}\right]\right) .
$$

\subsection{Effective Action for the Open String Sector}

After having discussed some aspects of the low energy theory corresponding to the closed sector of type IIB String Theory, we now turn to the open sector and provide an effective field theory description of D-branes and O-planes. For concreteness, we focus on type IIB orientifolds with O3- and O7-planes but our discussion readily extends to other configurations. 


\section{D3- and D7-Branes}

As we have explained in section 2.1, the fixed loci of the orientifold projection $\Omega(-1)^{F_{L}} \sigma$ are called orientifold planes and for the choice (2.3), these are O3and O7-planes usually carrying negative R-R and NS-NS charges. Therefore, as we will see in detail in section 7.1, we have to introduce D7-branes as well as a combination of D3-branes and background flux. Note that here, similarly to orientifold $\mathrm{O} p$-planes, the $p$ in $\mathrm{D} p$-brane denotes the number of spatial dimensions of the brane and the time direction is always included. These objects are thus $(p+1)$-dimensional.

In order to preserve four-dimensional Poincaré invariance, the D-branes have to be space-time filling, that is they occupy $\mathbb{R}^{3,1}$. For the D3-branes this implies that they are point-like in the compact space $\mathcal{X}$, and for the D7-branes it means they wrap a four-dimensional submanifold of $\mathcal{X}$. Furthermore, in order to preserve supersymmetry $[70,71,67]$, the D7-branes have to wrap

$$
\text { holomorphic four-cycles } \Gamma_{\mathrm{D} 7} \text { in } \mathcal{X}
$$

which are also called divisors. Let us remark that for type IIB orientifolds with O3- and O7-planes, we could also introduce $\mathrm{D} p$-branes for $p \neq 3,7$. However, the presence of such branes is highly restricted by the tadpole cancellation condition to be discussed in section 7.1, and such branes preserve supersymmetry only under special conditions. For most of the time we neglect this possibility and only come back to this point in section 7.3 .

\section{Gauge Flux on D7-Branes}

The D7-branes of interest to us are not only specified by a choice of divisor $\Gamma_{\mathrm{D} 7}$ in $\mathcal{X}$ but in addition by a choice of open string gauge flux $\bar{F}$. However, in order to preserve four-dimensional Lorentz invariance, we consider gauge flux $\bar{F}$ only in the internal space $\mathcal{X}$ and so we make the following ansatz for the total open string field strength $\mathbf{F}$

$$
\mathbf{F}=F+\bar{F} .
$$

Here, $F=d A$ denotes the field strength of the gauge field $A$ in $\mathbb{R}^{3,1}$ while $\bar{F}$ stands for the flux components in $\mathcal{X}$. Note that in general $\mathbf{F}$ is matrix valued and so we can expand $\mathbf{F}$ as

$$
\mathbf{F}=\sum_{A} \mathbf{F}^{A} T^{A}
$$


where $\left\{T^{A}\right\}$ is a basis of representation matrices. We will be more precise about this point later. Next, because $\mathbf{F}$ is not gauge invariant, we define

$$
\mathcal{F}=-i\left(l_{s}^{2} \mathbf{F}+2 \pi \varphi^{*} B_{2} \mathbb{1}\right),
$$

which is the gauge invariant open string field strength. In $(2.23), l_{s}$ denotes again the string length, $B_{2}$ is the NS-NS two-form and $\varphi^{*}$ is the pull-back from $\mathcal{X}$ to the divisor $\Gamma_{\mathrm{D} 7}$ the D7-brane is wrapping. Note that we will also employ the notation

$$
\overline{\mathcal{F}}=-i\left(l_{s}^{2} \bar{F}+2 \pi \varphi^{*} B_{2}^{(6)} \mathbb{1}\right)
$$

for the components of $\mathcal{F}$ in $\mathcal{X}$, and again for later reference, we define the following expression which will turn our to play an important role in the sequel

$$
\overline{\mathcal{F}}^{+}=-i\left(l_{s}^{2} \bar{F}+2 \pi \varphi^{*} B_{2}^{+} \mathbb{1}\right) .
$$

Furthermore, we note that similar to closed sector background fluxes, also the gauge fluxes have to be quantised properly. Denoting by $c_{1}\left(K_{\Gamma_{\mathrm{D} 7}}\right)$ the first Chern class of the canonical class of $\Gamma_{\mathrm{D} 7}$ (see for instance [59]), the quantisation condition reads $[50,72]$

$$
\frac{i \overline{\mathcal{F}}^{+}}{2 \pi}-\varphi^{*} B_{2}^{+}+\frac{1}{2} c_{1}\left(K_{\Gamma_{\mathrm{D} 7}}\right) \quad \in \quad H^{2}\left(\Gamma_{\mathrm{D} 7}, \mathbb{Z}\right)
$$

where $H^{2}\left(\Gamma_{\mathrm{D} 7}, \mathbb{Z}\right)$ denotes the second co-homology group of $\Gamma_{\mathrm{D} 7}$. This quantisation condition can have important consequences for instance in the case $c_{1}\left(K_{\Gamma_{\mathrm{D} 7}}\right) \neq 0$ [72], but it will not play a crucial role in this work.

Finally, we observe that the open string field strength $\mathbf{F}$ in 2.21$)$ is odd under the non-geometric part $\Omega(-1)^{F_{L}}$ of the orientifold projection, that is

$$
\Omega(-1)^{F_{L}} \mathbf{F}=-\mathbf{F}, \quad \Omega(-1)^{F_{L}} \mathcal{F}=-\mathcal{F} .
$$

\section{Curvature Two-Form}

Before presenting the effective action for D-branes and O-planes, we have to introduce one more piece of notation. In particular, the total curvature two-form of the tangent bundle of $\mathbb{R}^{3,1} \times \mathcal{X}$ we will denote by $\mathbf{R}$, which splits into $R$ and $\bar{R}$ defined on $\mathbb{R}^{3,1}$ respectively $\mathcal{X}$. For dimensional reasons, we then define

$$
\mathcal{R}=l_{s}^{2} \mathbf{R}=l_{s}^{2}(R+\bar{R}) .
$$

Furthermore, for a $\mathrm{D} p$-brane wrapping a manifold $\Gamma_{\mathrm{D} p}$ in $\mathcal{X}$ we denote the restriction of $\mathcal{R}$ to the tangent bundle of $\mathbb{R}^{3,1} \times \Gamma_{\mathrm{D} p}$ by $\mathcal{R}_{T}$ while the restriction to the normal bundle is usually denoted by $\mathcal{R}_{N}$. The same notation also applies to orientifold planes. 


\section{Effective Action}

We are now ready to write down the effective action for $\mathrm{D} p$-branes and $\mathrm{O} p$-planes. The kinetic terms for the gauge fields on the D-brane are encoded in the socalled Dirac-Born-Infeld (DBI) action and a corresponding version also exists for orientifold planes. The bosonic part of these actions reads $[73,74,35]^{5}$

$$
\begin{aligned}
& \mathcal{S}_{\mathrm{D} p}^{\mathrm{DBI}}=-\mu_{p} \int_{\mathrm{D} p} d^{p+1} \xi e^{-\phi} \sqrt{-\operatorname{det}\left(\left(\varphi^{*} g\right)_{i j}+\frac{i \mathcal{F}_{i j}}{2 \pi}\right)}, \\
& \mathcal{S}_{\mathrm{O} p}^{\mathrm{DBI}}=-Q_{p} \mu_{p} \int_{\mathrm{O} p} d^{p+1} \xi e^{-\phi} \sqrt{-\operatorname{det}\left(\left(\varphi^{*} g\right)_{i j}\right)}
\end{aligned}
$$

where $\xi^{i}$ with $i=0, \ldots, p$ are coordinates on the $\mathrm{D} p$-brane respectively $\mathrm{O} p$-plane, $\phi$ is the dilaton introduced previously and the integration is over the $(p+1)$ dimensional manifold the $\mathrm{D} p$-brane respectively $\mathrm{O} p$-plane is wrapping. Furthermore, $\left(\varphi^{*} g\right)_{i j}$ and $\mathcal{F}_{i j}$ in 2.29$)$ are matrices in the basis $\left\{\xi^{i}\right\}$ with $i=0, \ldots, p$, and we have defined the tension $\mu_{p}$ as well as the charge $Q_{p}$ as

$$
\mu_{p}=\frac{2 \pi}{l_{s}^{p+1}}, \quad Q_{p}=-2^{p-4} .
$$

Next, we turn to the Chern-Simons action of the $\mathrm{D} p$-branes and $\mathrm{O} p$-planes. These have been determined to take the following form [76-81] (see also [35])

$$
\begin{aligned}
& \mathcal{S}_{\mathrm{D} p}^{\mathrm{CS}}=-\mu_{p} \kappa_{p} \int_{\mathrm{D} p} \operatorname{ch}(\mathcal{F}) \wedge \sqrt{\frac{\hat{\mathcal{A}}\left(\mathcal{R}_{T}\right)}{\hat{\mathcal{A}}\left(\mathcal{R}_{N}\right)}} \wedge \bigoplus_{q} \varphi^{*} C_{q}, \\
& \mathcal{S}_{\mathrm{O} p}^{\mathrm{CS}}=-Q_{p} \mu_{p} \kappa_{p} \int_{\mathrm{O} p} \sqrt{\frac{\mathcal{L}\left(\mathcal{R}_{T} / 4\right)}{\mathcal{L}\left(\mathcal{R}_{N} / 4\right)}} \wedge \bigoplus_{q} \varphi^{*} C_{q},
\end{aligned}
$$

where, $\varphi^{*}$ denotes again the pull-back from $\mathcal{X}$ to the manifold the D-brane respectively O-plane is wrapping and $\mathcal{R}_{T}, \mathcal{R}_{N}$ stand for the restrictions of $\mathcal{R}$ to the tangent and normal bundle of this manifold. The definition of the Chern character $\operatorname{ch}(\mathcal{F})$, the $\hat{\mathcal{A}}$-genus and Hirzebruch polynomial $\mathcal{L}$ used in 2.31 can be found in appendix A. Furthermore, the sums in (2.31) run over $q=0,2,4,6,8,10$ and we

\footnotetext{
${ }^{5}$ Strictly speaking, the D-brane DBI action is only valid for a single D-brane with resulting $U(1)$ gauge theory. For stacks of D-branes with non-abelian gauge theories, a more involved version has to be employed [75].
} 
have introduced the signs ${ }^{6}$

$$
\kappa_{p}= \pm 1
$$

In the present case with O3- and O7-planes, it turns out that these have to be chosen as

$$
\kappa_{7}=+1, \quad \kappa_{3}=-1 .
$$

\footnotetext{
${ }^{6}$ These signs have already appeared in [82] where they were crucial in order to obtain the correct matching between the tadpole cancellation conditions of type IIB orientifolds with O9-/ O5-planes and the anomaly cancellation condition of the heterotic string. Similarly, as we will see in section 7.1, here the signs are important to match the D3-brane tadpole cancellation condition with F-theory.
} 


\section{Chapter 3}

\section{$\mathcal{N}=1$ Supergravity Description}

In this chapter, we will summarise some features of $\mathcal{N}=1$ supergravity theories in four dimensions. In particular, in section 3.1 we explain how the corresponding action can be characterised solely in terms of a Kähler potential $\mathcal{K}$, a holomorphic superpotential $W$, gauge kinetic functions $f_{a b}$ and possible Fayet-Iliopoulos terms $\xi_{a}[83,84]$. In section 3.2 , we then focus on type IIB orientifold compactifications with O3- and O7-planes and derive some results needed in the following.

\subsection{Generalities}

Let us begin with the general form of a $\mathcal{N}=1$ supergravity action in four spacetime dimensions. Denoting by $\star_{4}$ the four-dimensional Hodge- $\star$-operator, the action takes the following general form

$$
\mathcal{S}=\mathcal{S}_{\text {kin. }}-\int_{\mathbb{R}^{3,1}}\left(V_{F}+V_{D}\right) \star_{4} 1,
$$

where for convenience we have set Planck scale to $M_{\mathrm{Pl}}=1$. In the action (3.1) $\mathcal{S}_{\text {kin. }}$ denotes the kinetic part while the expressions $V_{F}$ and $V_{D}$ stand for the Frespectively D-term potential which we discuss in turn.

\section{The F-Term Potential}

The F-term potential $V_{F}$ is computed in terms of the holomorphic superpotential $W$, the Kähler potential $\mathcal{K}$ and the Kähler metric $G$ as

$$
V_{F}=e^{\mathcal{K}}\left(G^{\alpha \bar{\beta}} D_{\alpha} W D_{\bar{\beta}} \bar{W}-3|W|^{2}\right),
$$


where a summation over repeated indices is understood. Here and in the following, $\alpha$ runs over all holomorphic fields of the theory while $\bar{\beta}$ labels the anti-holomorphic ones. Furthermore, the so-called Kähler covariant derivative $D_{\alpha} W$ is computed as

$$
D_{\alpha} W=F_{\alpha}=\partial_{\alpha} W+\mathcal{K}_{\alpha} W, \quad \mathcal{K}_{\alpha}=\partial_{\alpha} \mathcal{K},
$$

where $\partial_{\alpha} \mathcal{K}$ denotes the derivative of the Kähler potential $\mathcal{K}$ with respect to the field labelled by $\alpha$. Also, the matrix $G^{\alpha \bar{\beta}}$ denotes the inverse of the Kähler metric $G_{\alpha \bar{\beta}}$ which is computed from the Kähler potential $\mathcal{K}$ in the following way

$$
G_{\alpha \bar{\beta}}=\partial_{\alpha} \partial_{\bar{\beta}} \mathcal{K}
$$

\section{The D-Term Potential}

After having discussed the F-term potential, we now turn to the D-term potential $V_{D}$. Denoting by $\operatorname{Re}\left(f^{a b}\right)$ the real part of the inverse of the gauge kinetic function $f_{a b}$, the D-term potential is written as

$$
V_{D}=\frac{1}{2} \operatorname{Re}\left(f^{a b}\right) D_{a} D_{b}
$$

where again a summation over repeated indices is understood and where $a, b$ label the gauge groups present in the theory. Furthermore, $D_{a}$ denotes the auxiliary D-fields which satisfy $G_{\alpha \bar{\beta}} \bar{X}_{a}^{\beta}=i \partial_{\alpha} D_{a}$ with $X_{a}^{\alpha}$ the corresponding holomorphic Killing vectors $[84,85]$.

In the case of linearly transforming scalars and diagonal gauge kinetic function $f_{a b}=f_{a} \delta_{a b}$, the D-term potential can be expressed as

$$
V_{D}=\sum_{a} \frac{1}{8 \operatorname{Re}\left(f_{a}\right)}\left(\mathcal{K}_{\alpha} T^{a} \phi_{\alpha}+\text { h.c. }\right)^{2},
$$

with $\mathcal{K}_{\alpha}$ the derivative of the Kähler potential $\mathcal{K}$ with respect to fields $\phi_{\alpha}$, and $T^{a}$ the representation matrices of the gauge symmetry.

Furthermore, let us also note that for an $U(1)$ symmetry the D-term $D_{a}$ can be shifted by a Fayet-Iliopoulos (FI) term $\xi_{a}$. (We will compute such FI terms in section 7.2.4.) With $Q_{a}^{m}$ the $U(1)_{a}$ charges of the canonically normalised matter fields $\Phi_{m}$, the D-term potential can then be expressed as

$$
V_{D}=\sum_{a} \frac{1}{2 \operatorname{Re}\left(f_{a}\right)}\left(\sum_{m} Q_{a}^{m}\left|\Phi_{m}\right|^{2}-\xi_{a}\right)^{2} .
$$




\subsection{Type IIB Orientifolds with O3-/O7-Planes}

After having given a general introduction to the action of $\mathcal{N}=1$ supergravity theories, in this section we are now going to specialise to (the closed sector of) type IIB orientifold compactifications with O3- and O7-planes.

\subsubsection{The Kinetic Part}

The kinetic terms in the action (3.1) will not be of importance for our following discussion, but for completeness we present the general form of the bosonic part

$$
\begin{aligned}
\mathcal{S}_{\text {kin. }}=-\frac{1}{2 \kappa_{4}^{2}} \int_{\mathbb{R}^{3,1}}\left(\mathrm{R}^{(4)} \star_{4} 1+2 G_{\alpha \bar{\beta}} D M^{\alpha} \wedge \star_{4} \overline{D M}^{\bar{\beta}}\right. \\
\left.\quad+\operatorname{Re}\left(f_{\kappa \lambda}\right) d V^{\kappa} \wedge \star_{4} d V^{\lambda}+\operatorname{Im}\left(f_{\kappa \lambda}\right) d V^{\kappa} \wedge d V^{\lambda}\right) .
\end{aligned}
$$

In this expression, $\mathrm{R}^{(4)}$ stands for the four-dimensional Ricci curvature scalar, $\kappa_{4}^{2}=M_{\mathrm{Pl}}^{-2}, M^{\alpha}=\left(U^{\hat{\kappa}}, T_{i}, G^{\hat{\imath}}, S\right)$ denotes the scalar components of the chiral multiplets given in table 2.2 , and $D M^{\alpha}$ stands for an appropriately covariantised derivative of these moduli. Note also, although not a kinetic term, in (3.8) we have included $d V^{\kappa} \wedge d V^{\lambda}$.

\subsubsection{The Kähler Potential and the Kähler Metric}

\section{Notation for the Kähler Sector}

Let us begin our discussion of the Kähler potential by introducing some notation. In particular, the triple intersection numbers $k_{I J K}$ of a Calabi-Yau manifold $\mathcal{X}$ in the basis $\left\{\omega_{I}\right\}$ introduced in table 2.1 are defined in the following way

$$
\frac{1}{l_{s}^{6}} \int_{\mathcal{X}} \omega_{I} \wedge \omega_{J} \wedge \omega_{K}=k_{I J K}, \quad I, J, K=1, \ldots, h^{1,1},
$$

where $I=i, \hat{\imath}$ collectively labels the $\sigma$-even and $\sigma$-odd basis elements of $H_{\bar{\partial}}^{1,1}(\mathcal{X})$. However, due to the splitting under the holomorphic involution $\sigma$, we find

$$
k_{i j \hat{k}}=0, \quad k_{\hat{\imath} \hat{\jmath} \hat{k}}=0,
$$


since the triple intersection number have to be invariant under $\sigma$. Next, because the Kähler form $J$ is even under the holomorphic involution, we expand $J$ in the following way

$$
J=t^{i} \omega_{i}, \quad i=1, \ldots, h_{+}^{1,1},
$$

where Einsteins sum convention is understood. We furthermore define the volume of an $\sigma$-even basis four-cycle $\gamma_{i} \in H_{4+}(\mathcal{X}, \mathbb{Z})$ as follows

$$
\tau_{i}=\frac{l_{s}^{-4}}{2} \int_{\gamma_{i}} J^{2}=\frac{1}{2} k_{i j k} t^{j} t^{k},
$$

and we express the overall volume of the compactification manifold $\mathcal{X}$ as

$$
\mathcal{V}=\frac{l_{s}^{-6}}{3 !} \int_{\mathcal{X}} J^{3}=\frac{1}{6} k_{i j k} t^{i} t^{j} t^{k}=\frac{1}{3} \tau_{i} t^{i}
$$

Now, in order to bring the Einstein-Hilbert term of the low energy effective action originating from String Theory compactifications into its standard form, one has to scale $J$ by $e^{-\phi / 2}$ where $\phi$ is the dilaton. The resulting action is said to be in Einstein frame, and the quantities defined above take the following form with the hat indicating the new frame

$$
\hat{t}^{i}=e^{-\frac{\phi}{2}} t^{i}, \quad \hat{\tau}_{i}=e^{-\phi} \tau_{i}, \quad \hat{\mathcal{V}}=e^{-\frac{3}{2} \phi} \mathcal{V}
$$

\section{Notation for the Complex Structure Moduli}

Let us now collect some notation which will become relevant for the complex structure moduli $U^{\hat{\kappa}}$. In particular, we denote a basis of $\sigma$-odd $(2,1)$-forms on $\mathcal{X}$ as

$$
\left\{\chi_{\hat{\kappa}}\right\} \in H_{\bar{\partial}-}^{2,1}(\mathcal{X}), \quad \hat{\kappa}=1, \ldots, h_{-}^{2,1},
$$

and a basis for $H_{\bar{\partial}-}^{1,2}(\mathcal{X})$ is obtained from 3.15 via complex conjugation. As it turns out, the basis $\left\{\chi_{\hat{\kappa}}\right\}$ can be computed from the holomorphic three-form $\Omega_{3}$ in the following way $[86,87]$

$$
\chi_{\hat{\kappa}}=D_{U^{\hat{\kappa}}} \Omega_{3}=\partial_{U^{\hat{\kappa}}} \Omega_{3}+\left(\partial_{U^{\hat{\kappa}}} \mathcal{K}^{\mathrm{CS}}\right) \Omega_{3},
$$

where the Kähler potential for the complex structure moduli $U^{\hat{\kappa}}$ takes the form

$$
\mathcal{K}^{\mathrm{CS}}=-\log \left(-\frac{i}{l_{s}^{6}} \int_{\mathcal{X}} \Omega_{3} \wedge \bar{\Omega}_{3}\right)
$$


Finally, for later reference let us define the matrix

$$
\mathcal{M}_{\hat{\kappa} \overline{\hat{\lambda}}}=\frac{1}{l_{s}^{6}} \int_{\mathcal{X}} \chi_{\hat{\kappa}} \wedge \bar{\chi}_{\hat{\lambda}}
$$

Note that here we have restricted ourselves to the $\sigma$-odd part of the third cohomology group since we are interested in the complex structure moduli for orientifold compactifications. However, these expression have their origin in compactifications without an orientifold projection where the index $\hat{\kappa}$ runs over the full co-homology group $H_{\bar{\partial}}^{2,1}(\mathcal{X})$ respectively $H_{\bar{\partial}}^{1,2}(\mathcal{X})$. For a more detailled discussion of this subject, see for instance [86,87].

\section{Kähler Potential}

We are now ready to state and discuss the Kähler potential for type II string compactifications. In particular, in $[60,64,88]$ the Kähler potential has been determined to take the following form

$$
\mathcal{K}=-\log (S+\bar{S})-2 \log \left(\hat{\mathcal{V}}+\frac{\hat{\xi}}{2}\right)-\log \left(-\frac{i}{l_{s}^{6}} \int_{\mathcal{X}} \Omega_{3} \wedge \bar{\Omega}_{3}\right)
$$

Note that the dependence of 3.19 on complex structure moduli solely resides in the holomorphic three-form $\Omega_{3}$, i.e.

$$
\Omega_{3}=\Omega_{3}\left(U^{\hat{\kappa}}\right) .
$$

Furthermore, we have written the overall volume $\mathcal{V}$ of the compactification manifold in Einstein frame which depends in a complicated way on the Kähler moduli $T_{i}$, and for $h_{-}^{1,1} \neq 0$ also on the moduli $G^{\hat{\imath}}$ as well as on the axio-dilaton $S$

$$
\mathcal{V}=\mathcal{V}\left(T_{i} \mid G^{\hat{\imath}}, S\right)
$$

Finally, in (3.19) we have included $\alpha^{\prime}$-corrections to the Kähler potential [88] which are characterised by

$$
\hat{\xi}=\frac{\xi}{g_{s}^{3 / 2}}, \quad \xi=-\frac{\zeta(3) \chi(\mathcal{X})}{2(2 \pi)^{3}}
$$

where $\zeta(z)$ denotes the Riemann $\zeta$-function, $\chi(\mathcal{X})$ stands for the Euler number of the internal manifold $\mathcal{X}$ and $g_{s}=e^{\phi}$ is the string coupling. We also mention that there are string loop-corrections to the Kähler metric [89-92], which will however not be include here. 


\section{First Derivative of the Kähler Potential}

After having given the expression for the Kähler moduli, let us now display the explicit results for the derivatives of (3.19) with respect to the moduli defined in (2.14), (2.15) and (2.16) which will be needed in section 7.2.4 when computing Fayet-Iliopoulos terms. The derivatives read (see also the appendices of [65] and [93])

$$
\begin{aligned}
\frac{\partial \mathcal{K}}{\partial S} & =-\frac{1}{2} \frac{e^{-\frac{\phi}{2}}}{\hat{\mathcal{V}}+\frac{\hat{\xi}}{2}}\left(e^{\frac{3}{2} \phi}(\hat{\mathcal{V}}+2 \hat{\xi})+\frac{1}{2} \frac{1}{l_{s}^{6}} \int_{\mathcal{X}}\left(B_{2}^{-}\right)^{2} \wedge J\right), \\
\frac{\partial \mathcal{K}}{\partial G^{\hat{\imath}}} & =-\frac{1}{2} \frac{e^{-\frac{\phi}{2}}}{\hat{\mathcal{V}}+\frac{\hat{\xi}}{2}}\left(\frac{i}{l_{s}^{4}} \int_{\gamma_{\hat{\imath}}} B_{2}^{-} \wedge J\right), \\
\frac{\partial \mathcal{K}}{\partial T_{i}} & =-\frac{1}{2} \frac{e^{-\frac{\phi}{2}}}{\hat{\mathcal{V}}+\frac{\hat{\xi}}{2}} \frac{1}{l_{s}^{2}} \int_{\Sigma^{i}} J .
\end{aligned}
$$

\section{Kähler Metric}

Next, we turn to the Kähler metric computed from the Kähler potential (3.19). In particular, for the case of interest in the following, namely $h_{-}^{1,1}=0$, the exact results are summarised in appendix $\mathrm{B}$, while for the situation $h_{-}^{1,1} \neq 0$ we would like to refer the reader to [93]. Using these expressions, we can summarise the generic form of the Kähler metric computed from (3.19) as

$$
\begin{aligned}
h_{-}^{1,1}=0: & G_{\alpha \bar{\beta}}=\left[\begin{array}{ccc}
G_{U \bar{U}} & 0 & 0 \\
0 & G_{T \bar{T}} & G_{T, \bar{S}} \\
0 & G_{S \bar{T}} & G_{S \bar{S}}
\end{array}\right] \stackrel{\xi \rightarrow 0}{\longrightarrow}\left[\begin{array}{ccc}
\circledast & 0 & 0 \\
0 & \circledast & 0 \\
0 & 0 & \circledast
\end{array}\right], \\
h_{-}^{1,1} \neq 0: & G_{\alpha \bar{\beta}}=\left[\begin{array}{cccc}
G_{U \bar{U}} & 0 & 0 & 0 \\
0 & G_{T \bar{T}} & G_{T \bar{S}} & G_{T \bar{G}} \\
0 & G_{S \bar{T}} & G_{S \bar{S}} & G_{S \bar{G}} \\
0 & G_{G \bar{T}} & G_{G \bar{S}} & G_{G \bar{G}}
\end{array}\right] \stackrel{\xi \rightarrow 0}{\longrightarrow}\left[\begin{array}{cccc}
\circledast & 0 & 0 & 0 \\
0 & \circledast & \circledast & \circledast \\
0 & \circledast & \circledast & \circledast \\
0 & \circledast & \circledast & \circledast
\end{array}\right],
\end{aligned}
$$

where we have indicated generically non-vanishing terms in the limit $\xi \rightarrow 0$ by $\circledast$. As we see from (3.24), in the case of vanishing $\alpha^{\prime}$-corrections the Kähler metric simplifies for $h_{-}^{1,1}=0$ but not for $h_{-}^{1,1} \neq 0$.

As we just mentioned, the explicit form of the Kähler metric involving $T_{i}$ and $S$ for the case $h_{-}^{1,1}=0$ can be found in appendix $\mathrm{B}$. Here, we only recall the following 
important result

$$
\mathcal{K}_{T_{i}} G^{T_{i} \bar{T}_{j}} \mathcal{K}_{\bar{T}_{j}}=3+3 \hat{\xi} \frac{\hat{\mathcal{V}}^{2}+7 \hat{\mathcal{V}} \hat{\xi}+\hat{\xi}^{2}}{(\hat{\mathcal{V}}-\hat{\xi})(2 \hat{\mathcal{V}}+\hat{\xi})^{2}}
$$

where Einsteins sum convention is understood. Note that in the limit of vanishing $\alpha^{\prime}$-corrections, that is $\xi=0$, the right hand side of equation (3.25) is a constant.

Let us finally turn to the Kähler metric for the complex structure moduli. As we have mentioned in a previous paragraph, the dependence of $\mathcal{K}$ on $U^{\hat{\kappa}}$ resides in the holomorphic three-form $\Omega_{3}$. Employing then equation (3.16) together with (3.18), we compute

$$
G_{U^{\hat{\kappa}} \bar{U}^{\hat{\lambda}}}=\partial_{U^{\hat{\kappa}}} \partial_{\bar{U}^{\hat{\lambda}}} \mathcal{K}=-\frac{\mathcal{M}_{\hat{\kappa} \hat{\lambda}}}{\frac{1}{l_{s}^{6}} \int_{\mathcal{X}} \Omega_{3} \wedge \bar{\Omega}_{3}} .
$$

\subsubsection{The Superpotential}

After having discussed the Kähler potential as well as the Kähler metric for type IIB orientifolds with O3- and O7-planes, we now turn to the superpotential.

\section{The Gukov-Vafa-Witten Superpotential}

We begin with the so-called Gukov-Vafa-Witten superpotential which describes the effect of background fluxes in the framework of an $\mathcal{N}=1$ supergravity action. More concretely, as we explain in detail in appendix $\mathrm{C}$, the fluxes for $F_{3}$ and $H_{3}$ introduced at the end of section 2.2 give rise to a potential in the effective four-dimensional action which can be described by the Gukov-Vafa-Witten superpotential $[94,95,58,88,96,60]$

$$
W_{\mathrm{GVW}}=\int_{\mathcal{X}} \Omega_{3} \wedge G_{3}, \quad G_{3}=\bar{F}_{3}-i S \bar{H}_{3}
$$

This superpotential turns out to be crucial for moduli stabilisation and we come back to this expression in chapter 6 .

\section{Non-Renormalisation Theorems}

Let us next observe that the Ramond-Ramond potentials, as the name suggests, are not invariant under gauge transformations: $C_{p} \rightarrow C_{p}+d \Lambda_{p-1}$ where $\Lambda_{p-1}$ 
is a $(p-1)$-form. Upon dimensional reduction, we then see from (2.14), (2.15) and (2.16) that the moduli $S, T_{i}$ and $G^{\hat{\imath}}$ can be shifted. Since in particular the superpotential at the perturbative level has to be invariant under such shifts, only the combinations 11

$$
(S+\bar{S})^{n}, \quad\left(T_{i}+\bar{T}_{i}\right)^{n}
$$

for $n \in \mathbb{Z}$ can appear in $W$. But, since the superpotential is a holomorphic quantity, the only allowed value for the exponent is $n=0$ which usually corresponds to treelevel contributions.

However, it is known that there are non-perturbative contributions to the superpotential which break the shift symmetry to a discrete subgroup. In particular, the allowed non-perturbative terms in the superpotential are of the form

$$
W_{\mathrm{np}}=W_{\mathrm{np}}\left(e^{-T_{i}}, e^{-S}\right) .
$$

Some of these contributions originate from so-called D-brane instantons which we will study in detail in chapter 5 .

\subsubsection{The Gauge Kinetic Function}

Although in this section we have focused on the closed sector of type IIB orientifold compactifications with O3- and O7-planes, let us conclude with a discussion of the gauge kinetic functions corresponding to the gauge theories on D3- respectively D7-branes.

As we have seen schematically already in equation (3.8), the real and imaginary part of the gauge kinetic function $f_{a b}$ appear in the action as

$$
\mathcal{S} \sim \int_{\mathbb{R}^{3,1}}\left(\operatorname{Re}\left(f_{a b}\right) F^{a} \wedge \star_{4} F^{b}+\operatorname{Im}\left(f_{a b}\right) F^{a} \wedge F^{b}\right)+\ldots,
$$

where $F^{a}$ denotes the field strength of the gauge theory labelled by $a$.

Let us investigate such terms for space-time filling D3-branes. In particular, performing an expansion of the square root in the DBI action (2.29) and writing out the Chern-Simons action (7.4), by comparing with (2.14) we can identify

$$
f_{\mathrm{D} 3}=S .
$$

\footnotetext{
${ }^{1}$ Two comments are in order. First, here we have not included the moduli $G^{\hat{\imath}}$ for which the discussion is somewhat more involved. Second, as we have seen for the Gukov-Vafa-Witten superpotential, in case $S$ is combined with $H_{3}$ and $F_{3}$ in an appropriate way, the shift symmetry of $S$ can be interpreted as a subgroup of the $S L(2, \mathbb{R})$ symmetry of type IIB supergravity.
} 
For space-time filling (supersymmetric) D7-branes, the discussion is more involved and utilises the D7-brane supersymmetry conditions to be discussed in section 4.4. We thus only quote the result which can be verified by employing the techniques developed during this work. For a D7-brane wrapping a four-cycle

$$
\Gamma_{\mathrm{D} 7}=m_{\mathrm{D} 7}^{I} \gamma_{I} \quad \in \quad H_{4}(\mathcal{X}, \mathbb{Z})
$$

where $\left\{\gamma_{I}\right\}$ is the basis of four-cycles introduced in table 2.1 and $m_{\mathrm{D} 7}^{I} \in \mathbb{Z}$ are the wrapping numbers, the corresponding gauge kinetic function can be expressed as

$$
f_{\mathrm{D} 7}=m_{\mathrm{D} 7}^{i} T_{i}+\ldots .
$$

The additional terms indicated by the ellipsis are due to the open string gauge flux $\overline{\mathcal{F}}^{+}$, gravitational terms originating from the $\hat{\mathcal{A}}$-genus in $(7.4)$, and from terms of the form $m_{\mathrm{D} 7}^{\hat{\imath}} \int_{\gamma_{\hat{\imath}}}\left(C_{4}+C_{2} \wedge B_{2}^{-}+\ldots\right)$ with $\left\{\gamma_{\hat{\imath}}\right\}$ the $\sigma$-odd four-cycles. However, such contributions will not be of importance here. 


\section{Chapter 4}

\section{Aspects of String Theory Model Building}

In this chapter, we are going to present an overview on aspects of String Theory model building in the context of type IIB orientifold compactifications with O3and O7-planes [35,97]. In particular, we state the rules for computing the chiral spectrum, we explain the cancellation of chiral anomalies via the generalised Green-Schwarz mechanism, comment on supersymmetry conditions for D7-branes, present the Freed-Witten anomaly cancellation condition and discuss K-theory constraints.

\subsection{Overview}

As we have already mentioned in the introduction, when constructing models in String Theory, a number of strong consistency conditions has to be satisfied. In the first section of this chapter, we will briefly summarise some of them.

- We begin with the tadpole cancellation conditions. Concerning the notation, the name tadpole is due to the fact that when these conditions are not satisfied, tadpole diagrams will appear in the effective low energy field theory which give rise to divergences.

From a geometric viewpoint, as explained in footnote 1 of chapter 2, the tadpole cancellation conditions are equivalent to the cancellation of charges in a compact space. On the other hand, from an effective field theory point of view, satisfying the tadpole cancellation conditions amounts to solving (part of) the equations of motion. In section 7.1, we will study this question in detail in the context of type IIB orientifolds with O3- and O7-planes. 
- In the next section of the present chapter, we are going to briefly state and explain the rules for computing the chiral spectrum originating from intersecting D7-branes with gauge flux. Note that since the chiral spectrum is counted by the index of the Dirac operator, via the Atiyah-Singer index theorem [98-100], it is related to certain topological quantities characterising the D-branes and O-planes.

- In section 4.3, we explain on general grounds how chiral anomalies are cancelled via the generalised Green-Schwarz mechanism [49,101-105]. In section 7.2, we then study this mechanism in detail for type IIB String Theory with O3- and O7-planes.

- In section 4.4, we will be more precise about the supersymmetry conditions for D7-branes with gauge flux,

- in section 4.5 we present the so-called Freed-Witten anomaly cancellation condition [50], and finally

- in section 4.6, we turn to the global $S U(2)$ anomaly known from Field Theory [106] in the context of String Theory.

Note that this list of String Theory constraints relevant for model building is surely not complete. In addition, there are for instance phenomenological conditions to be satisfied when engineering models reproducing the MSSM (or some extension thereof). Among these, there are the presence of precisely three generations, the absence of exotic matter and ensuring the correct values for the gauge couplings and the generation of Yukawa couplings. However, such issues will not be discussed here.

\subsection{Chiral Spectrum}

Let us now turn to the computation of the chiral spectrum in the context of type IIB orientifolds with D3-branes and D7-branes endowed with gauge flux.

Note that in this case, chiral matter will not originate from D3-branes because, as explained previously, chiral matter is related to topological quantities which are invariant under deformations of the geometry. Since the (generic) D3-branes under consideration are point-like in the internal space, they can always be moved away from other D3- or D7-branes thus making open strings stretched between those branes massive. Since under such changes of the geometry chiral matter would disappear, D3-branes do not give rise to the latter. 


\begin{tabular}{c||c} 
Representation & Multiplicity \\
\hline \hline$\left(\bar{N}_{a}, N_{b}\right)$ & $I_{a b}$ \\
$\left(N_{a}, N_{b}\right)$ & $I_{a^{\prime} b}$ \\
$\mathrm{~S}_{a}$ & $\frac{1}{2}\left(I_{a^{\prime} a}-2 I_{\mathrm{O} 7 a}\right)$ \\
$\mathrm{A}_{a}$ & $\frac{1}{2}\left(I_{a^{\prime} a}+2 I_{\mathrm{O} 7 a}\right)$
\end{tabular}

Table 4.1: Formulas for determining the chiral spectrum. Here $\left(N_{a}, N_{b}\right)$ denotes a bi-fundamental representation of the gauge group $G_{a} \times G_{b}$ while $S_{a}$ and $A_{a}$ stand for the symmetric respectively anti-symmetric representation of the gauge group $G_{a}$.

\section{Gauge Group}

Let us now discuss the possible gauge groups. We denote the gauge group on a stack of $N$ D7-branes without gauge flux by $G$, which for type II constructions usually is $U(N)$ or $S p(2 N)$ respectively $S O(2 N)$ in case the D-brane is invariant under the orientifold projection. If we turn on gauge flux $\bar{F}$ with structure group $\bar{H} \subset G$, then the observable gauge group $H$ is the commutant of $\bar{H}$ in $G$, that is

$$
H=\{h \in G: h \bar{h}=\bar{h} h \quad \forall \bar{h} \in \bar{H}\} .
$$

\section{Rules for Computing the Chiral Spectrum}

The rules for computing the chiral spectrum for type IIB orientifolds with D3- and D7-branes are summarised in table 4.1. The chiral index $I_{a b}$ used in this table is defined in the following way [107-109,35,97]

$$
I_{a b}=\frac{1}{l_{s}^{6}} \int_{\mathcal{X}}\left(\frac{\operatorname{ch}_{1}\left(\varphi_{*} \overline{\mathcal{F}}_{a}\right)}{N_{\mathrm{D} 7_{a}}}-\frac{\operatorname{ch}_{1}\left(\varphi_{*} \overline{\mathcal{F}}_{b}\right)}{N_{\mathrm{D} 7_{b}}}\right) \wedge\left[\Gamma_{\mathrm{D} 7_{a}}\right] \wedge\left[\Gamma_{\mathrm{D} 7_{b}}\right],
$$

where $\operatorname{ch}_{1}\left(\varphi_{*} \overline{\mathcal{F}}\right)$ denotes again the first Chern class of $\varphi_{*} \overline{\mathcal{F}}$ defined in (A.1), and $N_{\mathrm{D} 7_{a}}$ is the number of D7-branes in the stack $a$. Note that the somewhat unusual factors of $N_{\mathrm{D} 7}^{-1}$ are due to the fact, that we are counting representations. 11 Then, employing our definitions 2.13) and (2.25), we see that $B_{2}^{-}$cancels out in 4.2

\footnotetext{
${ }^{1}$ The chiral number of massless excitations between two D7-branes $a$ and $b$ is counted by the index $\widetilde{I}_{a b}=\frac{1}{l_{s}^{6}} \int_{\mathcal{X}}\left(\operatorname{ch}_{1}\left(\varphi_{*} \overline{\mathcal{F}}_{a}\right) \operatorname{ch}_{0}\left(\varphi_{*} \overline{\mathcal{F}}_{b}\right)-\operatorname{ch}_{0}\left(\varphi_{*} \overline{\mathcal{F}}_{a}\right) \operatorname{ch}_{1}\left(\varphi_{*} \overline{\mathcal{F}}_{b}\right)\right) \wedge\left[\Gamma_{\mathrm{D} 7_{a}}\right] \wedge\left[\Gamma_{\mathrm{D} 7_{b}}\right]$, which in the present case reduces to 4.2 when counting representations.
} 


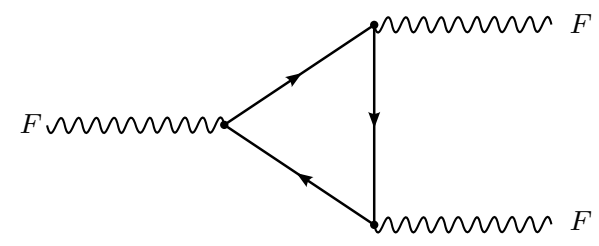

(a) Anomaly Diagram

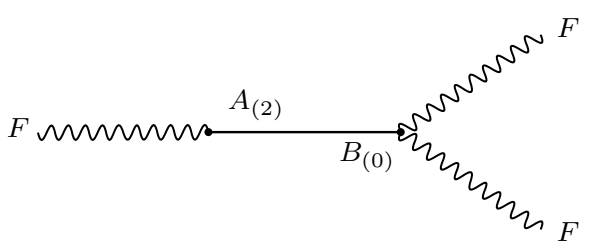

(b) Green-Schwarz Diagram

Figure 4.1: Anomaly and Green-Schwarz diagrams.

and so, as expected, only the quantised flux $\overline{\mathcal{F}}^{+}$contributes to the chiral index

$$
I_{a b}=\frac{1}{l_{s}^{6}} \int_{\mathcal{X}}\left(\frac{\operatorname{ch}_{1}\left(\varphi_{*} \overline{\mathcal{F}}_{a}^{+}\right)}{N_{\mathrm{D} 7_{a}}}-\frac{\operatorname{ch}_{1}\left(\varphi_{*} \overline{\mathcal{F}}_{b}^{+}\right)}{N_{\mathrm{D} 7_{b}}}\right) \wedge\left[\Gamma_{\mathrm{D} 7_{a}}\right] \wedge\left[\Gamma_{\mathrm{D} 7_{b}}\right] .
$$

\subsection{The Generalised Green-Schwarz Mechanism}

After having discussed how to determine the chiral matter content of a D-brane configuration, let us now turn to chiral anomalies. In particular, in type II String Theory constructions with D-branes, chiral anomalies originating from diagrams such as in figure 4.1(a) are cancelled via the generalised Green-Schwarz mechanism $[49,101-105]$.

The key observation for this mechanism to work is that in four dimensions a twoform $A_{(2)}$ and a scalar $B_{(0)}$ are dual to each other via the Hodge- $\star$-operation

$$
d A_{(2)} \sim \star_{4} d B_{(0)}
$$

Then, if there are couplings in the four-dimensional action of the form

$$
\operatorname{tr}(F) A_{(2)} \quad \text { and } \quad \operatorname{tr}\left(F^{2}\right) B_{(0)},
$$

one can construct diagrams which cancel the chiral anomalies. An example of such a Green-Schwarz diagram can be found in figure 4.1(b), and we discuss this mechanism in great detail in section 7.2 for type IIB orientifolds with O3- and O7-planes.

\subsection{Supersymmetry Conditions}

We now turn to the supersymmetry conditions for the D7-branes [70,71,67]. 


\section{D-Term Condition}

In the absence of matter fields for the D7-branes, or in the case of vanishing VEVs for these, in general the FI term in the D-term (3.7) is non-zero and supersymmetry is broken. Therefore, in order to preserve supersymmetry, the Fayet-Iliopoulos term has to vanish which leads to the following condition $[70,71,67]$

$$
\left.\left(J \wedge \operatorname{ch}_{1}(\overline{\mathcal{F}})\right)\right|_{\Gamma_{\mathrm{D} 7}}=0 \text {. }
$$

\section{Calibration Condition}

However, in addition to (4.6), there are further constraints on a D7-brane to preserve supersymmetry. In particular, the D7-brane has to satisfy a calibration condition $[71,67]$ which reads ${ }^{2}$

$$
d^{4} \xi \sqrt{\operatorname{det}\left(\left(\varphi^{*} g^{(6)}\right)_{i j}+\frac{i \overline{\mathcal{F}}_{i j}}{2 \pi}\right)}=\frac{1}{2} e^{-i \theta}\left(J-\frac{\overline{\mathcal{F}}}{2 \pi}\right) \wedge\left(J-\frac{\overline{\mathcal{F}}}{2 \pi}\right) .
$$

Note that we employed the notation introduced below $(2.29)$ as well as in equation (2.23), and that $g^{(6)}$ denotes the part of the metric $g$ in the internal space which is then pulled-back to the four-cycle the D7-brane is wrapping. Furthermore, the constant $\theta$ is a phase parametrising the unbroken supersymmetry. In the presence of an orientifold plane, as it turns out, in order for a D7-brane to respect the same supersymmetry as the former we have to require $\theta=0$. Together with (4.6), the calibration condition (4.7) then becomes

$$
d^{4} \xi \sqrt{\operatorname{det}\left(\left(\varphi^{*} g^{(6)}\right)_{i j}+\frac{i \overline{\mathcal{F}}_{i j}}{2 \pi}\right)}=\frac{1}{2}\left(J \wedge J+\frac{\overline{\mathcal{F}}}{2 \pi} \wedge \frac{\overline{\mathcal{F}}}{2 \pi}\right) .
$$

\subsection{Freed-Witten Anomaly Cancellation}

In addition to the chiral anomalies discussed above, in String Theory also the socalled Freed-Witten anomaly [50] has to be cancelled. This cancellation amounts to the requirement that the NS-NS field strength $H_{3}$ restricted to the D-brane has to be equal to the third Stiefel-Whitney class $W_{3}$ of the manifold the D-brane is wrapping

$$
\left.H_{3}\right|_{\text {D-brane }}=W_{3}(\text { D-brane }) \text {. }
$$

\footnotetext{
${ }^{2}$ Note that in the case of non-abelian gauge flux, here and in 4.8 appropriate traces have to be included.
} 
However, since in this work we are concerned with D7-branes wrapping divisors in a Calabi-Yau three-fold, it turns out that the third Stiefel-Whitney class is always vanishing [72] and so we arrive at

$$
\left.H_{3}\right|_{\text {D-brane }}=0 .
$$

Note finally, neglecting possible source terms such as lower-dimensional D-branes, equation (4.10) is just the Bianchi identity for the gauge invariant open string field strength $\mathcal{F}$ defined in 2.24 . That is

$$
0=d \overline{\mathcal{F}}=-i\left(l_{s}^{2} d \bar{F}+2 \pi \varphi^{*} d B_{2}^{(6)} \mathbb{1}\right)=-2 \pi i \varphi^{*} \bar{H}_{3} \mathbb{1} .
$$

\subsection{K-Theory Constraints}

To conclude this chapter, let us briefly discuss the so-called K-theory constraints. In particular, also in String Theory the global $S U(2)$ anomaly known from field theory [106] has to vanish. As it turns out, the vanishing of this anomaly is related to the fact that D-branes are not classified completely in terms of (co-)homology but rather in terms of K-theory $[107,47]$. In a compact space, also the $\mathbb{Z}_{2}$-valued K-theory charges not captured by (co-)homology have to be cancelled which then is related the cancellation of the $S U(2)$ anomaly.

These additional constraints can be detected via the probe-brane argument of [48]. More concretely, the cancellation of global $S p(2 N)$ anomalies translates into the requirement that the number of four-dimensional chiral fermions transforming in the fundamental representation of some $S p(2 N)$ factor has to be even. Taking then a probe D7-brane invariant under the orientifold projection carrying a $S p(2)$ gauge group, the condition for a model to be free of such anomalies reads

$$
\sum_{\mathrm{D} 7_{a}} N_{\mathrm{D} 7_{a}} I_{\mathrm{D} 7_{\text {probe }} \mathrm{D} 7_{a}} \in 2 \mathbb{Z}
$$

where $I_{a b}$ denotes the chiral index defined in equation (4.3), $N_{\mathrm{D} 7_{a}}$ is the again the number of D7-branes in the stack $a$ and the sum is over all such stacks. If this condition is satisfied for all probe D7-branes, also the K-theory charges are cancelled. 


\section{Chapter 5}

\section{D-Brane Instantons}

In this chapter, we are now going to discuss D-brane instantons in type II String Theories. The contribution of such instantons to terms in the superpotential will be important in chapter 6 when studying moduli stabilisation, and for a recent review on this subject we would like to refer the reader to [110].

\subsection{Prerequisites}

\section{Setup and Notation}

We begin by describing our setup for the study of D-brane instantons. As mentioned in the beginning of chapter 2, we consider compactifications of type II String Theory from ten to four dimensions on an orientifold of a Calabi-Yau three-fold $\mathcal{X}$. However, instantons are usually studied in euclidean space so we perform a Wick rotation and obtain

$$
\mathbb{R}^{9,1} \longrightarrow \mathbb{R}^{4} \times \mathcal{X}
$$

For such compactifications of type II String Theory to four dimensions, in analogy to D-branes, a $\mathrm{D} p$-brane instanton $\mathcal{E}$ is an object which is point-like in $\mathbb{R}^{4}$ and wraps a $(p+1)$-dimensional submanifold $\Gamma_{\mathcal{E}}$ in $\mathcal{X}$. Furthermore, these euclidean D-brane instantons are also called Ep-instantons where $E$ stands for euclidean and the $p$ indicates the dimension of the submanifold in $\mathcal{X}$. 


\section{Instanton Action and Supersymmetry Conditions}

The action corresponding to D-brane instantons is very analogous to the DBI and Chern-Simons action 2.29 respectively (2.31) for D-branes introduced in section 2.3. In particular, the only difference is that the integration is now only over the cycle $\Gamma_{\mathcal{E}}$ the instanton is wrapping in the internal space $\mathcal{X}$. We therefore write

$$
\begin{aligned}
& \mathcal{S}_{\mathrm{E} p}^{\mathrm{DBI}}=-\mu_{p} \int_{\mathrm{E} p} d^{p+1} \xi e^{-\phi} \sqrt{\operatorname{det}\left(\left(\varphi^{*} g\right)_{i j}+\frac{i \mathcal{F}_{i j}}{2 \pi}\right)}, \\
& \mathcal{S}_{\mathrm{E} p}^{\mathrm{CS}}=-\mu_{p} \kappa_{p} \int_{\mathrm{E} p} \operatorname{ch}(\mathcal{F}) \wedge \sqrt{\frac{\hat{\mathcal{A}}\left(\mathcal{R}_{T}\right)}{\hat{\mathcal{A}}\left(\mathcal{R}_{N}\right)}} \wedge \bigoplus_{q} \varphi^{*} C_{q},
\end{aligned}
$$

where the quantities used above are defined in the same way as for the a $\mathrm{D} p$-brane. As it turn out, the signs $\kappa_{p}$ important here are $\kappa_{(-1)}=+1$ and $\kappa_{3}=-1$.

Next, in order for the Ep-instantons to be supersymmetric, the same conditions as for D-branes apply. In particular, for E3-brane instantons, as explained in section 4.4. the flux $\overline{\mathcal{F}}_{\mathcal{E}}$ on an instanton is restricted by 4.6 and the four-cycle cycle $\Gamma_{\mathcal{E}}$ wrapped by the instanton has to be calibrated as stated in equation (4.8).

Let us now define the combined DBI and Chern-Simons action for a D $p$-brane instanton in the following way

$$
\mathcal{S}_{\mathrm{E} p}=\mathcal{S}_{\mathrm{E} p}^{\mathrm{DBI}}-i \mathcal{S}_{\mathrm{E} p}^{\mathrm{CS}}
$$

Specialising then to type IIB orientifolds with O3- and O7-planes, we see that the action for an $\mathrm{E}(-1)$-instanton is proportional to the axio-dilaton modulus $S$ defined in equation 2.14

$$
\mathcal{S}_{\mathrm{E}(-1)}=-2 \pi S
$$

On the other hand, the action of a single supersymmetric E3-instanton with $\overline{\mathcal{F}}_{\mathcal{E}}^{+}=0$ wrapped on a $\sigma$-even four-cycle $\gamma_{i}$ is related to the Kähler modulus $T_{i}$ defined in 2.15 as follows ${ }^{1}$

$$
\mathcal{S}_{\mathrm{E} 3}=-2 \pi\left(T_{i}+i \frac{l_{s}^{4}}{24} \chi\left(\gamma_{i}\right) C_{0}\right) \text {. }
$$

\footnotetext{
${ }^{1}$ The additional term proportional to the Euler number $\chi\left(\gamma_{i}\right)$ of the four-cycle $\gamma_{i}$ is due to the gravitational terms in the Chern-Simons action usually not included in the definition of the Kähler moduli.
} 


\section{Instanton Zero-Modes}

A subject important for this chapter are so-called instanton zero-modes. These can be thought of as massless excitations of open strings with at least one endpoint on the instanton. Let us discuss them in turn.

- The universal instanton zero-modes originate from strings with both endpoints on the same instanton and excitations in the four-dimensional space $\mathbb{R}^{4}$. The bosonic ones are usually denoted by $x^{\mu}$ with $\mu=0, \ldots, 3$ and describe the position of the instanton in $\mathbb{R}^{4}$. These zero-modes correspond to the four Goldstone bosons arising from the breakdown of four-dimensional translational invariance due to the instanton. Furthermore, in general there are four fermionic zero-modes associated with the breaking of the $\mathcal{N}=2$ supersymmetry in the bulk of the compactification manifold to $\mathcal{N}=1$ on a (supersymmetric) instanton. These zero-modes are usually denoted by $\Theta^{\alpha}$ and $\bar{\tau}^{\dot{\alpha}}$ with $\alpha, \dot{\alpha}=1,2[111,110]$.

Note that if a single instanton is invariant under the orientifold projection and carries a would-be $O(1)$ gauge group, the $\bar{\tau}^{\dot{\alpha}}$ zero-modes are projected out and one is left only with the $\Theta^{\alpha}$ modes corresponding to half of the $\mathcal{N}=1$ superspace [112-115, 111].

- Next, there can be so-called moduli zero-modes which arise from strings with both endpoints on the same instanton and excitations in the internal space $\mathcal{X}$. These zero-modes correspond to deformations of the cycle $\Gamma_{\mathcal{E}}$ the instanton is wrapping as well as to Wilson lines along this cycle. Note that the deformation modes are counted by co-homology classes of the form $H^{0}\left(\Gamma_{\mathcal{E}}, N\right)$ with $N$ the normal bundle of $\Gamma_{\mathcal{E}}$, while Wilson line moduli are encoded in $H_{\bar{\partial}}^{1,0}\left(\Gamma_{\mathcal{E}}\right)$. A cycle without these moduli zero-modes is called rigid.

For a single instanton not invariant under the orientifold projection, usually each deformation leads to a complex bosonic zero-mode as well as to four fermionic zero-modes.

- We now turn to the so-called charged instanton zero-modes. These arise as massless excitations of strings with one endpoint on the instanton and the other endpoint on a D-brane.

The chiral number of such zero-modes, that is say the number of left-handed minus the number of right-handed modes, is counted in a similar way as chiral matter between D-branes. In particular, in the case of type IIB orientifolds with O3- and O7-planes, in analogy to section 4.2, the chiral charged zeromodes between an E3-brane instanton and a D7-brane are counted by

$$
Z_{a \mathcal{E}}=\frac{1}{l_{s}^{6}} \int_{\mathcal{X}}\left(\operatorname{ch}_{1}\left(\varphi_{*} \overline{\mathcal{F}}_{a}^{+}\right)-\operatorname{ch}_{1}\left(\varphi_{*} \overline{\mathcal{F}}_{\mathcal{E}}^{+}\right)\right) \wedge\left[\Gamma_{\mathrm{D} 7_{a}}\right] \wedge\left[\Gamma_{\mathcal{E}}\right]
$$


Note that if there are only fermionic charged zero-modes present, in particular if there are only chiral or vector-like intersections between the instanton and a D-brane, these zero-modes are usually denoted by $\lambda$.

- Finally, let us mention that when multiple instantons are considered, there can be instanton zero-modes between different instantons. These are counted again in a similar way as matter between D-branes but we will not discuss such configurations here.

\section{Gauge vs. String Instantons}

To conclude this introductory section, we comment on the distinction between so-called gauge and string instantons.

- In particular, if an Ep-instanton is located directly on top of a stack of $\mathrm{D}(p+4)$ branes, the effect of this instanton in String Theory reproduces the effect of an ordinary gauge instanton in the four-dimensional theory originating from the D-branes. Such D-brane instantons are thus called gauge instantons, and we will study this point in detail in section 8.1.

- However, from the String Theory point of view, an instanton directly on top of a D-brane is a very special situation. A general Ep-instanton in String Theory is wrapping some cycle in $\mathcal{X}$ and will give rise to effects in the fourdimensional theory which usually cannot be understood from a pure field theoretical viewpoint. Therefore, Ep-instantons not on top of a D-brane are often called string instantons.

\subsection{Instanton Calculus}

In this section, we are going to explain how to compute the contribution of a Dbrane instanton to terms in the superpotential. For more details, please consult the original article [54] as well as the review [110].

\section{Correlation Functions in the Instanton Background}

We begin by discussing correlation functions in the instanton background [54]. In particular, the correlator involving $n$ fields $\Phi_{i}$ in the (single) instanton background is computed in the following way

$$
\left\langle\Phi_{1} \ldots \Phi_{n}\right\rangle_{\text {inst. }}=\int d\{\text { zero-modes }\} e^{\mathcal{S}_{\mathrm{E} p}+\mathcal{Z}_{0}^{\prime}} \sum_{\text {conf. ampl. }} \prod_{2 \times \lambda}\langle\widehat{\Phi}\rangle_{2 \times \lambda}^{\text {disc }}\langle\widehat{\Phi}\rangle^{1-\text { loop }} .
$$




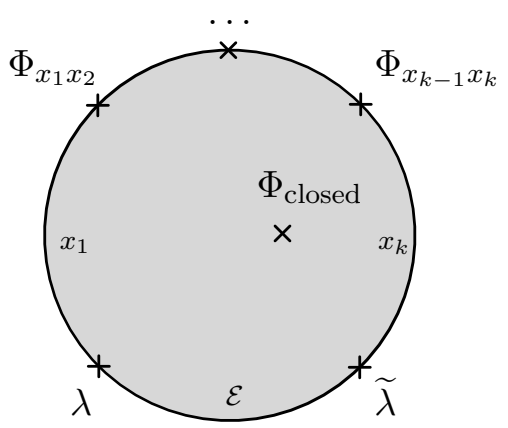

(a) Disc Amplitude

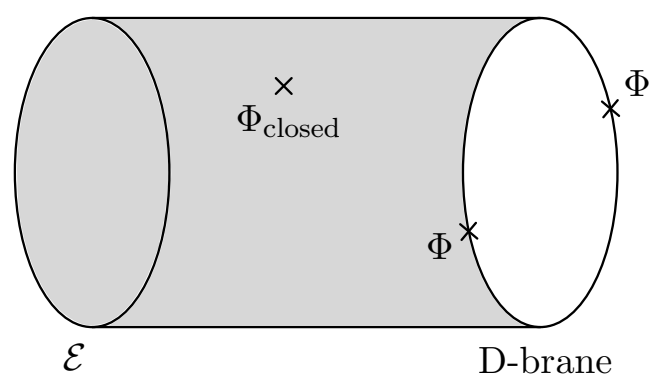

(b) One-Loop Amplitude

Figure 5.1: Example for a disc and a one-loop amplitude in the context of the D-brane instanton calculus. Please note the labels of the boundaries $\mathcal{E}, x_{1}$ and $x_{k}$ for the disc amplitude, and $\mathcal{E}$ respectively "D-brane" for the one-loop amplitude.

Note that this formula, as we will point out below, is already adjusted to the computation of holomorphic quantities such as the superpotential (see also section 3.2.3). Let us now explain how (5.8) is meant to be understood.

- We begin with the disc amplitude at the right of equation (5.8). The symbol $\widehat{\Phi}$ stands for the following combination of vertex operators

$$
\widehat{\Phi}=\Phi_{x_{1} x_{2}} \cdot \Phi_{x_{2} x_{3}} \cdot \ldots \Phi_{x_{k-2} x_{k-1}} \cdot \Phi_{x_{k-1} x_{k}} \cdot \Phi_{\text {closed }}
$$

where $\Phi_{x_{i} x_{i+1}}$ in the case $x_{i} \neq x_{i+1}$ is a boundary changing vertex operator and the $\left(x_{i}, x_{i+1}\right)$ labels the boundaries. Such operators correspond to bifundamental matter between D-branes $x_{i}$ and $x_{i+1}$. In the case $x_{i}=x_{i+1}$, the operator does not change the boundary of the disc and describes for instance D-brane moduli. Furthermore, note that also closed string vertex operator insertions in the interior of the disc are possible.

Next, the subscript " $2 \times \lambda$ " indicates that in addition to the vertex operators $\widehat{\Phi}$ precisely two charged instanton zero-modes have to be inserted. The reason for having two such zero-modes is given by the assignment of a factor of $\sqrt{g_{s}}$ to each of the $\lambda$ and the fact that a disc amplitude carries a factor of $g_{s}^{-1}$. Since the superpotential is holomorphic, it cannot solely depend on $g_{s}=\frac{S+\bar{S}}{2}$ which explains the two charged zero-modes for the disc amplitude since $g_{s}^{-1} \cdot \sqrt{g_{s}} \cdot \sqrt{g_{s}}=1$. However, instanton moduli zero-modes do not carry factors of $g_{s}$ and could therefore be inserted as well.

An example for a disc amplitude in the context of the D-brane instanton calculus can be found in figure $5.1(\mathrm{a})$. 
- We now turn to the one-loop amplitudes. These are cylinder or Möbius strip diagrams with one boundary the instanton under consideration and the other boundary a D-brane respectively an orientifold plane. The symbol $\widehat{\Phi}$ is defined in a similar way as for the disc diagram suitable adapted to the present case.

Note that for the one-loop amplitudes, no charged instanton zero-modes are to be inserted. The reason is that these amplitudes carry a factor of $g_{s}^{0}$, and for the superpotential to be holomorphic, no further dependence on $g_{s}$ originating for instance from the $\lambda$ 's is allowed. But, similarly as for the disc amplitude, moduli zero-modes can be inserted.

An example for a one-loop amplitude can be found in figure 5.1(b).

- The sum and the product in (5.8) are to be understood as follows. The sum is over all possible combinations of how the fields $\Phi_{i}$ can be distributed over factors of disc amplitudes and factors of one-loop amplitudes. However, each of the $\Phi_{i}$ has to appear precisely once.

- The exponential term in $(5.8)$ contains the instanton action $\mathcal{S}_{\mathrm{E} p}$ which corresponds to the vacuum disc amplitude of the instanton [116]. The symbol $\mathcal{Z}_{0}^{\prime}$ denotes the sum of all vacuum one-loop amplitudes involving the instanton, that is the sum over all annulus diagrams with boundary at the instanton and a D-brane as well as the Möbius strip amplitudes. The prime indicates that in these amplitudes the massless states, i.e. the zero-modes, have to be removed since these are taken care of explicitly.

Let us also mention that the exponential originates from a sum over all possible products of vacuum amplitudes with appropriate symmetry factors of $1 / n$ ! included [116].

- Finally, the integral in $(5.8)$ is over all instanton zero-modes. Note that in the case of fermionic zero-modes, the integral is only non-vanishing if these zero-modes are absorbed in disc or one-loop diagrams.

From the correlation functions given in equation (5.8), one can now construct the instanton generated terms in the superpotential. Let us however emphasise that one has to check whether the disc and one-loop diagrams are actually nonvanishing. In most cases, this is only possible if a CFT description is available.

\section{Discussion of Instanton Zero-Modes}

We now discuss in some detail how to deal with instanton zero-modes. As just mentioned, we have to absorb them in disc and one-loop diagrams and integrate over them. We explain how this can be achieved for each type of zero-modes in 
turn.

- We start with the universal zero-modes. The bosonic modes $x^{\mu}$ and the two fermionic ones $\Theta^{\alpha}$ give rise to the $\mathcal{N}=1$ superspace integration measure

$$
\int d^{4} x d^{2} \Theta
$$

which is needed for contributions to the superpotential. However, the additional modes $\bar{\tau}^{\dot{\alpha}}$ require a different treatment.

In the case of a gauge instanton, the $\bar{\tau}^{\dot{\alpha}}$ modes can be absorbed in disc diagrams and the integration gives rise to the fermionic ADHM constraints known from field theory [117-120]. In the case of a single instanton directly on top of a single brane (sometimes called Petersson instanton), a configuration which does not correspond to a proper gauge instanton, the additional fermionic zeromodes can also be absorbed [121]. Furthermore, the $\bar{\tau}^{\dot{\alpha}}$ zero-modes might get lifted by background fluxes $[111,122]$.

However, especially for string instantons, in the following we will assume that the instanton is invariant under the orientifold projection and carries a wouldbe $O(1)$ gauge group. As mentioned earlier, in this case the $\bar{\tau}^{\dot{\alpha}}$ zero-modes are projected out.

- Next, we turn to the moduli zero-modes. These are usually hard to absorb but can be lifted, i.e. become massive, by flux or curvature on the moduli space (see [110] and references therein). In the following we will simply assume that the instanton wraps a rigid cycle meaning that the moduli zero-modes are absent.

- Finally, as mentioned above, the charged zero-modes have to be absorbed in disc or one-loop diagrams. In the case of a gauge instanton, there usually are $4 n$ bosonic charged zero-modes and $2 n$ fermionic ones where $n \in \mathbb{Z}^{+}$. Roughly speaking, the fermionic zero-mode integration leads to a factor of the form $\prod_{i} \Phi_{i}$ in front of the exponential where $\Phi_{i}$ are certain matter fields, while the bosonic integration gives a factor of $\left(\prod_{i} \Phi_{i}\right)^{-2}$ [51]. Thus, the schematic form of the generated term in the superpotential originating from a gauge instanton reads 2

$$
W_{\text {gauge }} \sim \frac{1}{\prod_{i} \Phi_{i}} e^{\mathcal{S}_{\mathrm{E} p}}
$$

We will be much more precise about gauge instantons in section 8.1.

\footnotetext{
${ }^{2}$ Note that 5.11 is a very schematic expression in the $\alpha^{\prime} \rightarrow 0$ limit. The precise form of the contribution of course depends on the specific setup under consideration.
} 
On the other hand, string instantons usually have no bosonic charged zeromodes and so their contribution to the superpotential takes the schematic form

$$
W_{\text {string }} \sim \prod_{i} \Phi_{i} e^{\mathcal{S}_{\mathrm{E} p}}
$$

\section{Concluding Remarks}

Before closing this chapter, there are two main points we would like to emphasise to the reader.

- First, as stated in equation (5.6), the instanton action of a E3-instanton wrapping some basis four-cycle $\gamma_{i}$ is proportional to the corresponding Kähler modulus $T_{i}$. Therefore, as already stressed in (3.29), in the context of type IIB orientifolds with O3- and O7-planes, Kähler moduli will appear as exponentials in the non-perturbative superpotential. This observation will be important in chapter 6 when discussing moduli stabilisation.

- Second, for a non-vanishing contribution of an instanton to the superpotential, one has to carefully absorb the instanton zero-modes. As we will show in section 8.2 , this can lead to interesting structures for terms in the superpotential. 


\section{Chapter 6}

\section{Moduli Stabilisation}

In this chapter, we turn to the discussion of moduli stabilisation in the context of type IIB String Theory compactifications with O3- and O7-planes. In particular, in section 6.1 we explain the so-called KKLT scenario [55] and in section 6.2, following the work of $[56,57]$, we present the so-called LARGE Volume Scenario (LVS) which is an extension of the KKLT construction.

\subsection{The KKLT Scenario}

We start with the discussion of a scenario developed by Kachru, Kallosh, Linde and Trivedi (KKLT) [55] and thereby introduce the concepts needed also for the LARGE Volume Scenario to be studied in section 6.2.

\subsubsection{General Analysis}

\section{Assumptions}

Before actually getting started, let us make clear our assumptions for this section. In the end, we comment on relaxing these.

- We do not take into account $\alpha^{\prime}$-corrections to the Kähler sector of the Kähler potential $(3.19)$, that is we set $\xi=0$. In view of equation (3.22), neglecting these corrections does not mean we are considering manifolds with vanishing Euler characteristic but rather that we work at tree-level in $\alpha^{\prime}$.

- We assume that a manifold and an orientifold projection can be chosen such that $h_{-}^{1,1}=0$. From table 2.2 , we then see that there are no $G^{\hat{\imath}}$-moduli present. 
- We basically neglect the open sector of type IIB orientifold compactifications. In particular, we ignore the presence of an MSSM-like sector engineered by D-branes.

\section{Background Fluxes}

As mentioned at the end of section 2.2, in general we are allowed to turn on background fluxes in the internal manifold $\mathcal{X}$, that is we can have non-vanishing VEVs for the field strengths $F_{3}=d C_{2}$ and $H_{3}=d B_{2}$ [58]. In equation (3.27), we stated the so-called Gukov-Vafa-Witten superpotential [94, 95, 58, 88, 96, 60]

$$
W_{\mathrm{GVW}}=\int_{\mathcal{X}} \Omega_{3} \wedge G_{3}, \quad G_{3}=\bar{F}_{3}-i S \bar{H}_{3},
$$

which describes such flux compactifications from a four-dimensional point of view. Note that by abuse of notation, $\bar{G}_{3}$ denotes the complex conjugate of $G_{3}$ while $\bar{F}_{3}$ and $\bar{H}_{3}$ stand for the real fluxes originating from $F_{3}$ respectively $H_{3}$. For later reference, let us then compute the F-terms corresponding to this superpotential. In particular, we find

$$
\begin{aligned}
& F_{U^{\hat{\kappa}}}=\int_{\mathcal{X}} \chi_{\hat{\kappa}} \wedge G_{3}, \\
& F_{S}=-\frac{1}{S+\bar{S}} \int_{\mathcal{X}} \Omega_{3} \wedge \bar{G}_{3},
\end{aligned}
$$

\section{Moduli Stabilisation: Step I}

We are now ready to discuss the first step of moduli stabilisation in the KKLT Scenario. From (3.24) we recall the form of the Kähler metric for $h_{-}^{1,1}=0$ in the case $\xi=0$. Suppressing the indices on the fields, we have the following blockdiagonal matrix

$$
G_{\alpha \bar{\beta}}=\left[\begin{array}{ccc}
G_{U \bar{U}} & 0 & 0 \\
0 & G_{S \bar{S}} & 0 \\
0 & 0 & G_{T \bar{T}}
\end{array}\right] .
$$

Computing the F-term potential (3.2) by using equation 3.25 with $\xi=0$, we see that $V_{F}$ possesses a so-called no-scale property and can be brought into the form

$$
\begin{aligned}
V_{F} & =e^{\mathcal{K}}\left(G^{U \bar{U}} F_{U} \bar{F}_{\bar{U}}+G^{S \bar{S}} F_{S} \bar{F}_{\bar{S}}+G^{T \bar{T}} \mathcal{K}_{T} \mathcal{K}_{\bar{T}}\left|W_{\mathrm{GVW}}\right|^{2}-3\left|W_{\mathrm{GVW}}\right|^{2}\right) \\
& =e^{\mathcal{K}}\left(G^{U \bar{U}} F_{U} \bar{F}_{\bar{U}}+G^{S \bar{S}} F_{S} \bar{F}_{\bar{S}}\right) .
\end{aligned}
$$


Note that this expression is positive semi-definite and does not depend on the Kähler moduli $T_{i}$. The minimum of (6.4) is reached when all F-terms $F_{U}$ and $F_{S}$ are vanishing. From (6.2), we infer that this is the case when the $(1,2)$ - and $(3,0)$-components of $G_{3}$ are vanishing, i.e.

$$
G_{3}^{1,2}=G_{3}^{3,0}=0
$$

The way to interpret this result goes as follows [55]. First, one chooses some quantised fluxes $\bar{F}_{3}$ and $\bar{H}_{3}$ in $G_{3}$. Since the distinction between holomorphic and anti-holomorphic coordinates depends on the complex structure, the potential (6.4) will fix the complex structure moduli $U^{\hat{\kappa}}$ and the axio-dilaton $S$ in such a way that the $G_{3}$-flux has vanishing $(1,2)$ - and $(3,0)$-components. For a generic choice of flux, usually all moduli $U^{\hat{\kappa}}$ and $S$ can be stabilised in this way. (For reviews see for instance $[123,124,35,125]$.)

Although not verified explicitly, this will be our assumption in the following and the value of the Gukov-Vafa-Witten superpotential in the minimum will be denoted by $W_{0}$

$$
\left.W_{\mathrm{GVW}}\right|_{\text {min. }}=W_{0}
$$

\section{Moduli Stabilisation: Step II}

After the complex structure moduli and the axio-dilaton have been stabilised via fluxes, we now turn to the Kähler moduli. But, as we can see from (6.4), the $T_{i}$ do not appear in the F-term potential and therefore no mass term for these fields is generated.

However, so far we have only considered tree-level contributions to the superpotential. As explained around equation (3.29) in section 3.2.3, due to their shift symmetry, the Kähler moduli appear in $W$ via non-perturbative effects. As we have seen in chapter 5 , these can originate from D-brane instantons wrapping four-cycles in $\mathcal{X}$, but also from gaugino condensates on a stack of D7-branes also wrapping four-cycles

$$
\Gamma_{\Lambda}=m_{\Lambda}^{i} \gamma_{i}, \quad m_{\Lambda}^{i} \in \mathbb{Z},
$$

where $\left\{\gamma_{i}\right\}$ is again a basis of $H_{4+}(\mathcal{X}), m_{\Lambda}^{i}$ are the corresponding wrapping numbers and $\Lambda$ labels the non-perturbative contributions. The generic form of such terms then reads

$$
W_{\mathrm{np}}=\sum_{\Lambda} \mathcal{A}_{\Lambda}(U) e^{-a_{\Lambda} T_{\Lambda}} \quad \text { with } \quad T_{\Lambda}=m_{\Lambda}^{i} T_{i}
$$


where we utilised equation $(5.6)$. The prefactor $\mathcal{A}_{\Lambda}$ contains the one-loop amplitudes mentioned in equation (5.8) which depend only on the complex structure moduli $U^{\hat{\kappa}}$, and the coefficients $a_{\Lambda}$ in the exponent read

$$
a_{\Lambda}=\frac{2 \pi}{N}, \quad \quad N \in \mathbb{Z}^{+} .
$$

Note that for D-brane instantons, $N$ is usually one but in the case of gaugino condensates, $N$ indicates the rank of the gauge group.

Our next step is to stabilise the Kähler moduli. More concretely, we treat $U^{\hat{\kappa}}$ and $S$ as fixed, and combine $W_{0}$ defined in (6.6) with the small corrections (6.8) to

$$
W=W_{0}+\sum_{\Lambda} \mathcal{A}_{\Lambda}(U) e^{-a_{\Lambda} T_{\Lambda}}
$$

Since now the superpotential also depends on the Kähler moduli, we have to reconsider (6.4). Taking into account that the F-terms for $U$ and $S$ are vanishing, we find for the scalar potential as well as for its derivative that

$$
\begin{aligned}
V_{F} & =e^{\mathcal{K}}\left(G^{T \bar{T}} F_{T} \bar{F}_{\bar{T}}-3|W|^{2}\right), \\
\partial_{T} V_{F} & =e^{\mathcal{K}}\left(D_{T}\left(G^{T \bar{T}} F_{T} \bar{F}_{\bar{T}}\right)-3 F_{T} \bar{W}\right),
\end{aligned}
$$

where $D_{T}$ denotes the Kähler covariant derivative introduced in (3.3) with respect to the Kähler moduli $T$. Note that the explicit form of (6.11) can be obtained by setting $\xi=0$ in equation (B.10). From equation (6.12), we infer that the potential has an extremum if all F-terms for the Kähler moduli are vanishing, i.e.

$$
F_{T}=0
$$

Of course, we furthermore have to check that this solution really is a minimum but let us for the moment assume this is indeed the case.

When stabilising all moduli via vanishing F-terms, the F-term potential in the minimum takes the following form

$$
\left.V_{F}\right|_{\text {min. }}=e^{\mathcal{K}}\left(0-3|W|^{2}\right) \simeq-3 e^{\mathcal{K}}\left|W_{0}\right|^{2} \leq 0 .
$$

In conclusion, in the KKLT Scenario the complex structure moduli $U^{\hat{\kappa}}$ and the axio-dilaton $S$ are stabilised via $G_{3}$-flux, and the Kähler moduli are stabilised via non-perturbative effects. The minimum is a supersymmetric AdS minimum. 


\section{Uplift}

However, experimental data $[126,127]$ suggests that we are living in a de Sitter vacuum with a positive cosmological constant. In order to go from an Anti-de Sitter to a de Sitter vacuum, one can for instance add anti-D3-branes in a strongly warped region of the compactification manifold. An explicit example of such a region is a so-called Klebanov-Strassler throat [128] generated by background $\bar{F}_{3}$ and $\bar{H}_{3}$ fluxes which leads to an uplift potential of the form

$$
V_{\text {uplift }} \simeq \frac{1}{\hat{\mathcal{V}}^{2}}\left(e^{-\frac{2 \pi}{3 g_{s}} \frac{K}{M}}\right)^{4}
$$

where $\mathcal{V}$ is again the overall volume of the compactification manifold, $g_{s}$ is the string coupling and $K, M \in \mathbb{Z}$ are certain flux quanta. However, also other uplift mechanisms, for instance via D-terms, are possible.

\subsubsection{An Example}

Let us now illustrate the ideas presented above for a simple toy model. In particular, we focus on a manifold with a single Kähler modulus $T$, i.e. we assume $h_{+}^{1,1}=1$ (in addition to $h_{-}^{1,1}=0$ ). For the overall volume of the compactification manifold $\mathcal{X}$ this implies

$$
\hat{\mathcal{V}}=(\hat{\tau})^{\frac{3}{2}}
$$

where $\hat{\tau}=\operatorname{Re}(T)$ and where we have set a possible proportionality constant to one. Furthermore, we assume that by a generic choice of $G_{3}$-flux, all complex structure moduli $U^{\hat{\kappa}}$ as well as the axio-dilaton $S$ have been stabilised in a supersymmetric way.

We will now be more precise about the Kähler modulus. As we have mentioned above equation (6.13), an extremum of the potential (6.11) is reached when the Fterm for $T$ is vanishing. In order to compute $F_{T}$, let us display the superpotential in the present case as well as the first derivative of the Kähler potential with respect to $T$

$$
\begin{aligned}
W & =W_{0}+\mathcal{A} e^{-a T}, \quad W_{0} \in \mathbb{R}, \quad \mathcal{A} \in \mathbb{R}, \\
\mathcal{K}_{T} & =-\frac{3}{T+\bar{T}} .
\end{aligned}
$$

Note that we have assumed $W_{0}$ and $\mathcal{A}$ to be real which simplifies our discussion but does not change the general result. The vanishing of the F-term for the Kähler 
modulus then reads

$$
0=F_{T} \simeq-a \mathcal{A} e^{-a T}-\frac{3}{T+\bar{T}} W_{0}
$$

The solution to this equation (and its complex conjugate) cannot be expressed in a closed form but can be written as

$$
\operatorname{Im}(T)=0, \quad \hat{\tau} e^{-a \hat{\tau}}=-\frac{3 W_{0}}{2 a \mathcal{A}},
$$

where again $\operatorname{Re}(T)=\hat{\tau}$. Therefore, via (6.19) the Kähler modulus is stabilised in a supersymmetric way.

However, note that in order for the supergravity description to be valid, effects of the order of the string scale have to be neglected which means that we are considering volumes $\tau \gg 1$. Furthermore, to trust the non-perturbative effects we have to ensure that $a \hat{\tau}>1$. By comparing with the last expression in (6.19), we see that this implies

$$
\left|W_{0}\right| \ll 1
$$

which can be achieved by a certain fine-tuning of the background fluxes. ! $^{1}$ We remark that the prefactor $\mathcal{A}$ for the instanton contributions is assumed to be of order one.

To finish the discussion of our toy model, let us plot the potential in the situation where the complex structure moduli and axio-dilaton have been stabilised via $F_{U}=F_{S}=0$ and where $\operatorname{Im}(T)=0$. The resulting expression can for instance be determined with the help of equation $\mathrm{B} .10$ for $\xi=0$ and reads

$$
V_{F} \sim \frac{4 a^{2} \mathcal{A}^{2}}{3} \frac{e^{-2 a \hat{\tau}}}{\hat{\tau}}+4 a \mathcal{A} \frac{e^{-a \hat{\tau}}}{\hat{\tau}^{2}} W
$$

where we have set the constant prefactor originating from $e^{\mathcal{K}}$ to one. In figure 6.1(a) the potential for certain specific values of the parameters $m, \mathcal{A}$ and $W_{0}$ can be found. We see that there is indeed a minimum of the potential at $\left.\hat{\tau}\right|_{\text {min. }} \simeq 16.82$ with value $\left.V\right|_{\text {min. }} \simeq-5 \times 10^{-20}$. In figure 6.1(b), the uplift potential (6.15) has been included which leads to a minimum at $\left.\hat{\tau}\right|_{\text {min. }} \simeq 16.88$ with value $\left.V\right|_{\text {min. }} \simeq$ $+6 \times 10^{-22}$.

\footnotetext{
${ }^{1}$ However, the landscape of String Theory vacua $[31,129,41,42]$ exhibits many solutions for $F_{U}=F_{S}=0$ leading to very many distinct values for $W_{0}$. Therefore, in the String Theory landscape points with $\left|W_{0}\right| \ll 1$ can indeed be found which avoids the fine-tuning of $W_{0}$.
} 


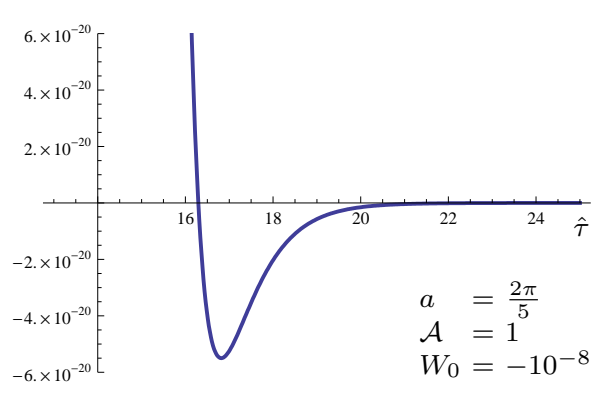

(a) KKLT potential 6.21

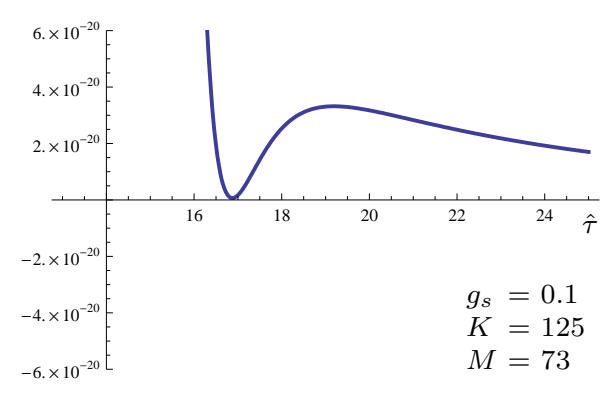

(b) KKLT potential 6.21 together with uplift potential 6.15

Figure 6.1: In figure 6.1(a) the KKLT potential 6.21) for a specific choice of parameters is shown. In figure 6.1(b), the result for the uplift potential (6.15) added to (6.21) can be found.

\subsubsection{Discussion}

After having explained the basic strategy of the KKLT Scenario and having presented a simple toy model, let us now discuss this approach in some more detail.

- We recall that we have first stabilised the complex structure moduli $U^{\hat{\kappa}}$ and the axio-dilaton $S$ via the Gukov-Vafa-Witten superpotential (6.1), but have ignored the contribution (6.8) from non-perturbative effects. In a second step, treating $U^{\hat{\kappa}}$ and $S$ as fixed, we stabilised the Kähler moduli with the help of (6.8).

However, as it has been pointed out for instance in [130,131], such a two-step procedure is not always justified because also the non-perturbative effects depend via the prefactor $\mathcal{A}(U)$ on $U^{\hat{\kappa}}$.

- We have explicitly neglected $\alpha^{\prime}$-corrections to the Kähler potential $\mathcal{K}$, that is we have set $\xi=0$ in 3.19 . As a consequence, the F-term potential exhibits a no-scale structure removing the dependence of the Kähler moduli in 6.4). When taking into account $\alpha^{\prime}$-corrections, the Kähler metric contains off-diagonal terms (see (3.24)) which render the two-step procedure, especially for the axio-dilaton, ambiguous.

- In our explicit toy model, we have seen that in order to obtain a reliable minimum for the Kähler moduli, we have to fine-tune the contribution from the fluxes (6.20). This observation can also be made in more general constructions with more than one Kähler modulus and may be regarded as a disadvantage of the KKLT construction. 
- By assumption, we have only considered the closed string sector and neglected an open sector with D-branes possibly realising the MSSM. It is usually assumed that the open sector is independent of the closed sector and that the MSSM can be engineered after the moduli have been stabilised. However, in section 8.2 we will see that this statement is not true since there is an complex interplay between the open and closed sector.

In particular, when the MSSM is realised via D-branes, at least one of the Kähler moduli does not appear in the superpotential via non-perturbative effects. Therefore, a KKLT scenario with $h^{1,1}=1$ is not possible. We will be much more precise about this point in chapter 9 .

- We have assumed that $h_{-}^{1,1}=0$ implying the absence of the moduli $G^{\hat{\imath}}$. When considering $h_{-}^{1,1} \neq 0$, we can see from (3.24) that the Kähler metric becomes much more complicated - even in the case of $\xi=0$. This situation has been analysed in [93].

- Finally, the uplift procedure is slightly ambiguous. Because of the anti-D3branes, supersymmetry is broken explicitly and so it is a priori not clear if the resulting theory can be described in terms of $\mathcal{N}=1$ supergravity. However, a careful analysis reveals that effectively the anti-D3-branes can be described by non-linear supersymmetry transformations [132].

But there are alternative possibilities. For instance, in [133] it was analysed under which conditions de Sitter vacua can be realised. It turns out that when more general contributions to the superpotential are considered, de Sitter vacua can be obtained without an explicit uplift potential.

In summary, the KKLT Scenario is a crucial step towards the stabilisation of all moduli which is important for phenomenology. However, this scenario features a number of drawbacks which ask for improvement.

\subsection{The LARGE Volume Scenario}

To remedy some of the shortcomings of the KKLT Scenario, we now turn to the so-called LARGE Volume Scenario $[56,57]$ and discuss this construction in detail. 


\subsubsection{General Analysis}

\section{Assumptions}

Before we start, let us first make clear our assumptions for the LARGE Volume Scenario and point out the differences to the KKLT approach.

- In contrast to KKLT, we take into account $\alpha^{\prime}$-corrections to the Kähler potential $\mathcal{K}$. In particular, we assume that the Euler number of the compactification manifold $\mathcal{X}$ is non-vanishing. Furthermore, as we will explain, we assume $\chi(\mathcal{X})<0$.

- We assume that a manifold and an orientifold projection can be chosen such that $h_{-}^{1,1}=0$ implying the absence of the $G^{\hat{\imath}}$-moduli. A discussion of the LARGE Volume Scenario for $h_{-}^{1,1} \neq 0$ can be found in [93].

- We basically neglect the open sector of type IIB orientifolds compactifications, that is we ignore the presence of an MSSM-like sector engineered by D-branes meaning that the D-term potential vanishes $V_{D}=0$. However, in chapter 9. we explicitly consider an open sector and study its implication for moduli stabilisation.

- Finally, we also make a more technical assumption about the overall volume of the compactification manifold $\mathcal{X}$. In particular, we assume that $\mathcal{V}$ can be expressed in terms of the four-cycle volumes $\tau_{i}$ in the following way

$$
\mathcal{V}=\eta_{\mathrm{b}}\left(\tau_{\mathrm{b}}\right)^{\frac{3}{2}}-\sum_{\tilde{\imath}=2}^{h_{+}^{1,1}} \eta_{\tilde{\imath}}\left(\tau_{\tilde{\imath}}\right)^{\frac{3}{2}}, \quad \quad \eta_{\mathrm{b}}, \eta_{\tilde{\imath}} \in \mathbb{R}^{+}
$$

Note that we have singled out the $\tau_{1}=\tau_{\mathrm{b}}$ volume where $b$ stands for big. As we can see from $6.22, \tau_{\mathrm{b}}$ can take any large value while the remaining four-cycle volumes $\tau_{\tilde{\imath}}$ are bounded due to the requirement that $\mathcal{V}$ has to be positive. Manifolds for which the volume can be expressed in this way have been called swiss-cheese manifolds $[134,45,91]$ since $\tau_{\mathrm{b}}$ can be thought of as describing the overall volume of the cheese while, due to the minus sign, the $\tau_{\tilde{\imath}}$ describe small holes.

In the course of our discussion, we will explain why this form of the volume is necessary for the LARGE Volume Scenario, and in the end we comment on generalisations of 6.22 . 


\section{The LARGE Volume Limit}

As the name of the present section suggests, we are interested in configurations where the overall volume $\mathcal{V}$ of the compactification manifold is very large compared to the string scale. This will allow us to perform an expansion of various quantities in powers of $1 / \hat{\mathcal{V}}$ since

$$
\frac{1}{\hat{\mathcal{V}}} \ll 1
$$

We are also going to assume that the real part of the Kähler moduli $T_{i}$ exhibits the following approximate scaling with the overall volume $\mathcal{V}$

$$
\hat{\tau}_{\mathrm{b}} \sim \hat{\mathcal{V}}^{\frac{2}{3}}, \quad a_{\tilde{\imath}} \hat{\tau}_{\tilde{\imath}} \sim \log \hat{\mathcal{V}}, \quad \tilde{\imath}=2, \ldots, h_{+}^{1,1},
$$

where $a_{\tilde{\imath}} \sim \frac{2 \pi}{N}$. Let us however emphasise that these assumptions about $\mathcal{V}$ and the scaling of $\hat{\tau}_{i}$ are only made to simplify our presentation of the LARGE Volume Scenario. Later, (6.23) and (6.24) will arise as dynamical solutions when minimising the F-term potential.

\section{Superpotential and F-Terms}

Let us now turn to the superpotential. Similarly to the KKLT construction, we turn on background $G_{3}$-flux giving rise to the Gukov-Vafa-Witten superpotential (6.1). Furthermore, we consider non-perturbative contributions to the superpotential arising from D-brane instantons or gaugino condensates. However, since the limits 6.23 and $(6.24)$ imply $\tau_{\mathrm{b}} \gg \tau_{\tilde{\imath}}$, the non-perturbative effects corresponding to $\tau_{\mathrm{b}}$ can be neglected compared to $\hat{\tau}_{\tilde{\imath}}$. The full superpotential therefore reads

$$
W=W_{\mathrm{GVW}}+W_{\mathrm{np}} \simeq \int_{\mathcal{X}} \Omega_{3} \wedge G_{3}+\sum_{\Lambda} \mathcal{A}_{\Lambda}(U) e^{-a_{\Lambda} T_{\Lambda}},
$$

where we explicitly exclude the big modulus $\tau_{\mathrm{b}}$ in $T_{\Lambda}$, i.e.

$$
T_{\Lambda}=\sum_{\tilde{\imath}=2}^{h_{+}^{1,1}} m_{\Lambda}^{\tilde{\imath}} T_{\tilde{\imath}}
$$

Let us now consider the F-term potential. In particular, using the limits 6.23) and (6.24) as well as the Kähler metrics given in (3.26) and (B.8), we find that

$$
\begin{array}{lll}
G^{U \bar{U}} F_{U} \bar{F}_{\bar{U}} \sim \mathcal{O}\left(\hat{\mathcal{V}}^{0}\right), & G^{S \bar{T}} F_{S} \bar{F}_{\bar{T}} & \sim \mathcal{O}\left(\hat{\mathcal{V}}^{-1}\right), \\
G^{S \bar{S}} F_{S} \bar{F}_{\bar{S}} \sim \mathcal{O}\left(\hat{\mathcal{V}}^{0}\right), & G^{T \bar{T}} F_{T} \bar{F}_{\bar{T}}-3|W|^{2} \sim \mathcal{O}\left(\hat{\mathcal{V}}^{-1}\right) .
\end{array}
$$




\section{Moduli Stabilisation I: Complex Structure Moduli and Axio-Dilaton}

After having determined the scalings 6.27), we can now write down the F-term potential at leading order in $1 / \hat{\mathcal{V}}$. We find

$$
V_{F}=e^{\mathcal{K}}\left(G^{U \bar{U}}\left[F_{\mathrm{GVW}}\right]_{U}\left[\bar{F}_{\mathrm{GVW}}\right]_{\bar{U}}+G^{S \bar{S}}\left[F_{\mathrm{GVW}}\right]_{S}\left[\bar{F}_{\mathrm{GVW}}\right]_{\bar{S}}\right)+\mathcal{O}\left(\hat{\mathcal{V}}^{-3}\right)
$$

where we used that $e^{\mathcal{K}} \sim \hat{\mathcal{V}}^{-2}$ and where the subscript "GVW" indicates that only the flux superpotential is used to compute the F-term. Note that this expression is similar to 6.4 obtained in the KKLT Scenario. However, here we have a controlled expansion making this form stable against $\alpha^{\prime}$-corrections.

Now, similarly to the KKLT case, we minimise the F-term potential which fixes the complex structure moduli $U^{\hat{\kappa}}$ and the axio-dilaton $S$ via the vanishing F-terms computed from the Gukov-Vafa-Witten superpotential, this time at leading order in $1 / \hat{\mathcal{V}}$. For the following, we now treat these as fixed and use

$$
F_{U}=0+\mathcal{O}\left(\hat{\mathcal{V}}^{-1}\right), \quad F_{S}=0+\mathcal{O}\left(\hat{\mathcal{V}}^{-1}\right)
$$

Finally, as before, we denote $W_{\mathrm{GVW}}$ in this minimum by $W_{0}$.

\section{Moduli Stabilisation II: Kähler Moduli}

Next, we employ $(6.29)$ and determine the scalar F-term potential at order $1 / \hat{\mathcal{V}}^{3}$. Utilising the exact computation (B.10) for $F_{U}=F_{S}=0$ and performing the limit $\hat{\mathcal{V}} \gg 1$ with the superpotential

$$
W \simeq W_{0}+\sum_{\Lambda} \mathcal{A}_{\Lambda} e^{-a_{\Lambda} T_{\Lambda}}
$$

we find the following expression

$$
\begin{aligned}
V_{F}=\frac{g_{s}}{2} \varrho[\quad & \frac{4}{\hat{\mathcal{V}}} \sum_{\Lambda, \Delta} \widehat{\operatorname{Vol}}\left(\Gamma_{\Lambda} \cap \Gamma_{\Delta}\right) a_{\Lambda} \mathcal{A}_{\Lambda} a_{\Delta} \overline{\mathcal{A}}_{\Delta} e^{-a_{\Lambda} T_{\Lambda}-a_{\Delta} \bar{T}_{\Delta}} \\
& +\frac{2}{\hat{\mathcal{V}}^{2}} \sum_{\Lambda} \widehat{\operatorname{Vol}}\left(\Gamma_{\Lambda}\right) a_{\Lambda}\left(\mathcal{A}_{\Lambda} e^{-a_{\Lambda} T_{\Lambda}} \bar{W}_{0}+\text { c.c. }\right) \\
& \left.+\frac{3}{4} \frac{\hat{\xi}}{\hat{\mathcal{V}}^{3}}\left|W_{0}\right|^{2}\right]+\mathcal{O}\left(\hat{\mathcal{V}}^{-4}\right),
\end{aligned}
$$


with $\Lambda, \Delta$ labelling the non-perturbative contributions to the superpotential and where we have defined the quantities

$$
\begin{aligned}
& \frac{1}{\varrho}=-\frac{i}{l_{s}^{6}} \int_{\mathcal{X}} \Omega_{3} \wedge \bar{\Omega}_{3}, \quad \widehat{\operatorname{Vol}}\left(\Gamma_{\Lambda} \cap \Gamma_{\Delta}\right)=m_{\Lambda}^{i} m_{\Delta}^{j} k_{i j k} \hat{t}^{k}, \\
& \widehat{\operatorname{Vol}}\left(\Gamma_{\Lambda}\right)=\hat{\tau}_{\Lambda}=m_{\Lambda}^{i} \hat{\tau}_{i} .
\end{aligned}
$$

Note that the latter two relations denote the volumes (in Einstein frame) of the intersection of two four-cycles $\Gamma_{\Lambda}$ and $\Gamma_{\Delta}$ respectively the volume of the four-cycle $\Gamma_{\Lambda}$.

After having determined the F-term potential at order $1 / \hat{\mathcal{V}}^{3}$ with the complex structure moduli and the axio-dilaton stabilised, let us make a rough estimate about the stabilisation procedure. We will be much more precise in the following paragraph when discussing an explicit example.

- First, for simplicity we assume that $\widehat{\operatorname{Vol}}\left(\Gamma_{\Lambda} \cap \Gamma_{\Delta}\right) \sim \delta_{\Lambda \Delta}$ which removes the dependence of $\operatorname{Im}(T)$ from the first line in (6.31).

- Then, we see that $\operatorname{Im}(T)$ only appears in the second line of (6.31). Minimising this term with respect to the imaginary part of the Kähler moduli will fix these such that

$$
\left(\mathcal{A}_{\Lambda} e^{-a_{\Lambda} T_{\Lambda}} \bar{W}_{0}+\text { c.c. }\right) \quad \longrightarrow \quad-2 e^{-a_{\Lambda} \hat{\tau}_{\Lambda}}\left|\mathcal{A}_{\Lambda} W_{0}\right|
$$

- Finally, we employ the scaling (6.24) in the resulting potential which leads to the following generic form

$$
V_{F} \sim-\frac{\sigma}{\hat{\mathcal{V}}^{3}} \sqrt{\log \hat{\mathcal{V}}}-\frac{\log \hat{\mathcal{V}}}{\hat{\mathcal{V}}^{3}}+\frac{\hat{\xi}}{\hat{\mathcal{V}}^{3}},
$$

where we have neglected all numerical prefactors but have been careful about the signs of the three terms. In particular, we have defined

$$
\sigma=\operatorname{sign}\left[\widehat{\operatorname{Vol}}\left(\Gamma_{\Lambda} \cap \Gamma_{\Delta}\right)\right]
$$

- In figures 6.2 we have plotted the potential (6.34) for the choices $\sigma= \pm 1$ and $\hat{\xi}= \pm 2$. We see that for $\{\sigma, \hat{\xi}\}=\{+1,+2\}$ there is a minimum for small values of $\hat{\mathcal{V}}$, and that for $\{\sigma, \hat{\xi}\}=\{-1,+2\}$ there is a minimum at large values of $\hat{\mathcal{V}}$. The other two possibilities do not give rise to a minimum. 


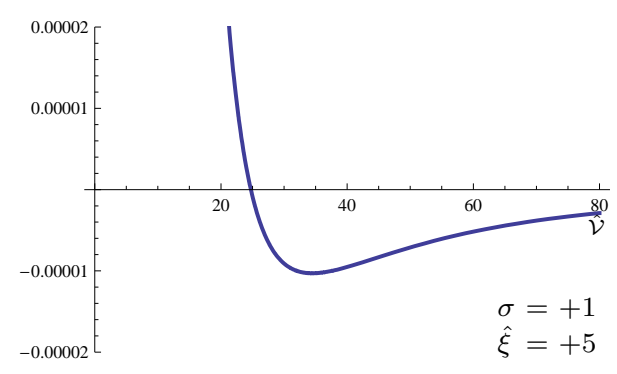

(a) Sign Choice 1

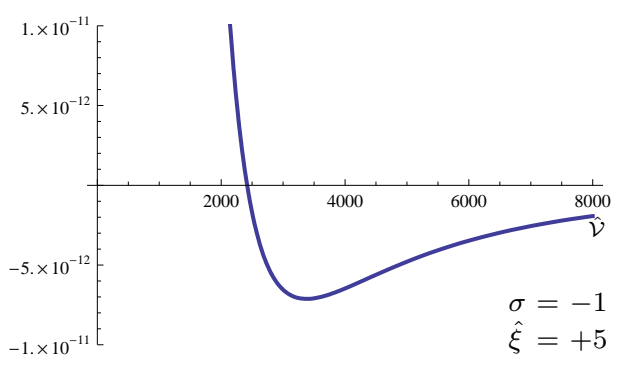

(c) Sign Choice 3

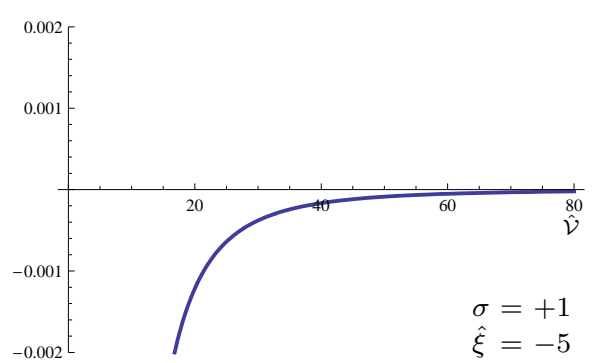

(b) Sign Choice 2

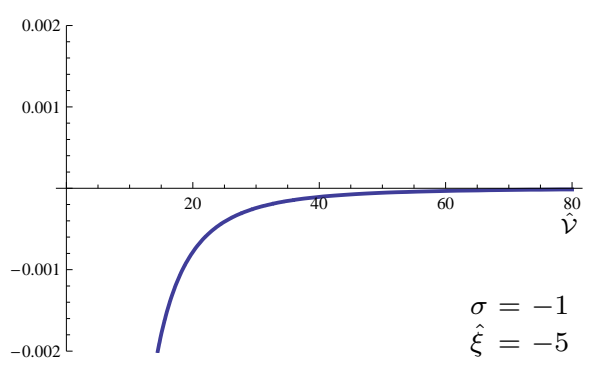

(d) Sign Choice 4

Figure 6.2: Plot of the potential (6.34) for different choices of $\sigma$ and $\hat{\xi}$. Please note the different ranges in these plots.

\section{Summary}

Let us now briefly summarise our findings of this subsection.

- We have a assumed that volume of the compactification manifold has a swisscheese structure and we have neglected non-perturbative effects corresponding to the big modulus. In this way, we can identify $\tau_{\mathrm{b}}$ with $\mathcal{V}$.

- We have assumed (and later verified) that the overall volume $\hat{\mathcal{V}}$ can be stabilised at very large values. This allowed us to perform an expansion of the F-term potential in orders of $1 / \hat{\mathcal{V}}$.

- At leading order in $1 / \hat{\mathcal{V}}$, the complex structure moduli and the axio-dilaton can be stabilised by the vanishing of the F-terms computed from the GukovVafa-Witten superpotential.

- At subleading order in $1 / \hat{\mathcal{V}}$, after taking into account $F_{U}=F_{S}=0$, we have computed the resulting scalar potential in (6.31). Using then the scaling (6.24) and some simplifying assumptions, we were able to deduce the dependence of $V_{F}$ on $\mathcal{V}$. 
- Leaving open some signs, we plotted this dependence and found that there is a minimum of $V_{F}$ for two choices of $\mathcal{V}$. One corresponds to small and the other to large values of $\mathcal{V}$. However, in order to obtain this minimum we have to assume

$$
\chi(\mathcal{X})<0, \quad \widehat{\operatorname{Vol}}\left(\Gamma_{\Lambda} \cap \Gamma_{\Delta}\right)<0 .
$$

Note that the second requirement is satisfied for swiss-cheese manifolds 6.22 with instantons on the small cycles. This justifies our assumptions from the beginning of this section.

\subsubsection{An Example}

After having explained the LARGE Volume Scenario on a general level, let us now present an explicit example where these ideas are realised. We expect however that the same features are present also in more general constructions.

\section{Setup}

The standard example primarily studied in the literature [56] is the Calabi-Yau manifold $\mathbb{P}_{[1,1,1,6,9]}[18]$ which has a co-dimension three $\mathbb{Z}_{3}$-singularity [135-137]. Its resolution introduces a second Kähler modulus $T_{\mathrm{s}}$ so that the volume becomes ${ }^{2}$

$$
\mathcal{V}=\frac{1}{9 \sqrt{2}}\left(\tau_{\mathrm{b}}^{\frac{3}{2}}-\tau_{\mathrm{s}}^{\frac{3}{2}}\right)
$$

where the subscript in $\tau_{\mathrm{s}}$ stands for small. Note that the only way to make $\mathcal{V}$ large is to take $\tau_{\mathrm{b}}$ large, the other choice would lead to a negative volume $\mathcal{V}$ which is not well-defined. The non-trivial Hodge numbers for this manifold are $\left(h^{1,1}, h^{2,1}\right)=(2,272)$, and we assume that an orientifold projection can be chosen such that $h_{+}^{1,1}=2, h_{-}^{1,1}=0$. The Euler number of this space is found as

$$
\chi(\mathcal{X})=\chi\left(\mathbb{P}_{[1,1,1,6,9]}[18]\right)=-540,
$$

implying that the first requirement from 6.36 is satisfied.

In the limit we are interested in, namely $\mathcal{V} \gg 1$, non-perturbative contributions involving $\tau_{\mathrm{b}}$ can be neglected and only $\tau_{\mathrm{s}}$ will play a role. Assuming that the

\footnotetext{
${ }^{2}$ More concretely, the hypersurface $\mathbb{P}_{[1,1,1,6,9]}[18]$ can be seen as a $\mathbb{T}^{2}$ fibration over $\mathbb{P}^{2}$, and the small divisor with volume $\tau_{\mathrm{s}}$ is $\mathbb{P}^{2}$. The big divisor with volume $\tau_{\mathrm{b}}$ is a linear combination of $\mathbb{P}^{2}$ and $\pi^{*}(l)$ where the latter denotes the pull-back of the class of a line $l$ in $\mathbb{P}^{2}[135,72]$.
} 
complex structure moduli and the axio-dilaton have been stabilised via the GukovVafa-Witten superpotential at order $1 / \hat{\mathcal{V}}^{2}$, and denoting the latter in the minimum by $W_{0}$, we have

$$
W \simeq W_{0}+\mathcal{A} e^{-a T_{\mathrm{s}}},
$$

where we have set the wrapping number $m^{\mathrm{s}}$ in the exponent to one. Of course, in view of chapter 5, here we have assumed that there exists an E3-instanton for which all zero-modes can be absorbed leading to a non-vanishing contribution, respectively that there exists a stack of D-branes with gaugino condensation. But, since in our particular example the small cycle is rigid and we do not take into account matter D7-branes, E3-brane instantons generically will contribute to the non-perturbative superpotential. ${ }^{3}$

\section{The F-Term Potential for the Kähler Moduli}

With this information about the compactification manifold and the non-perturbative contribution to the superpotential, from $(\mathrm{B} .10)$ we can now determine the F-term potential for the Kähler moduli. To do so, with the help of (B.4) we compute

$$
\widehat{\operatorname{Vol}}\left(\Gamma_{\mathrm{s}} \cap \Gamma_{\mathrm{s}}\right)=k_{\mathrm{ss} k} \hat{t}^{k}=\frac{1}{2}\left(\frac{\partial^{2} \hat{\mathcal{V}}}{\partial \tau_{\mathrm{s}}^{2}}\right)^{-1}=-6 \sqrt{2} \sqrt{\hat{\tau}_{\mathrm{s}}}
$$

and see that the second requirement from $(6.36)$ is satisfied. The F-term potential at order $1 / \hat{\mathcal{V}}^{3}$ is then determined to be of the form

$$
V_{F}=\frac{g_{s}}{2} \varrho\left[24 \sqrt{2} a^{2}|\mathcal{A}|^{2} \frac{\sqrt{\hat{\tau}_{\mathrm{s}}}}{\hat{\mathcal{V}}} e^{-2 a \hat{\tau}_{\mathrm{s}}}+2 a \frac{\hat{\tau}_{\mathrm{s}}}{\hat{\mathcal{V}}^{2}}\left(\mathcal{A} e^{-a T_{\mathrm{s}}} \bar{W}_{0}+\text { c.c. }\right)+\frac{3}{4} \frac{\hat{\xi}}{\hat{\mathcal{V}}^{3}}\left|W_{0}\right|^{2}\right] \text {. }
$$

Next, since the imaginary part of $T_{\mathrm{s}}$ appears only in the second term of (6.41), the minimum of $V_{F}$ is reached when this term is as negative as possible which fixes the axion $\operatorname{Im}\left(T_{\mathrm{s}}\right)$. The potential then reads

$$
V_{F}=\frac{g_{s}}{2} \varrho\left[\lambda a^{2}|\mathcal{A}|^{2} \frac{\sqrt{\hat{\tau}_{\mathrm{s}}}}{\hat{\mathcal{V}}} e^{-2 a \hat{\tau}_{\mathrm{s}}}-4 a|\mathcal{A}|\left|W_{0}\right| \frac{\hat{\tau}_{\mathrm{s}}}{\hat{\mathcal{V}}^{2}} e^{-a \hat{\tau}_{\mathrm{s}}}+\frac{3}{4} \frac{\hat{\xi}}{\hat{\mathcal{V}}^{3}}\left|W_{0}\right|^{2}\right],
$$

where in order to generalise our discussion in the next paragraph, we have introduced a constant $\lambda$ which in the present case reads

$$
\lambda=24 \sqrt{2} .
$$

\footnotetext{
${ }^{3}$ The reason why the small cycle is rigid can be understood with the help of footnote 2, In particular, as mentioned before, the small divisor is $\mathbb{P}^{2}$ which is indeed rigid.
} 


\section{Analytic Analysis}

Let us now perform an analytical analysis of the potential (6.42). In particular, we are interested in the minima of $V_{F}\left(\hat{\tau}_{\mathrm{s}}, \hat{\mathcal{V}}\right)$. Note that we have performed the change of variables $\hat{\tau}_{\mathrm{b}} \rightarrow \hat{\mathcal{V}}$ and that the axion $\operatorname{Im}\left(T_{\mathrm{b}}\right)$ does not appear in $V_{F}$. We come back to this point in the next subsection.

In any case, setting to zero the derivative of the F-term potential (6.42) with respect to $\hat{\mathcal{V}}$ leads to the following expression

$$
\hat{\mathcal{V}}=\frac{4\left|W_{0}\right| \sqrt{\hat{\tau}_{\mathrm{s}}}}{\lambda a|\mathcal{A}|} e^{a \hat{\tau}_{\mathrm{s}}}\left(1 \pm \sqrt{1-\frac{9 \hat{\xi} \lambda}{64 \hat{\tau}_{\mathrm{s}}^{3 / 2}}}\right)
$$

Computing the derivative of the F-term potential with respect to $\hat{\tau}_{\mathrm{s}}$ and imposing it to vanish gives to the following result

$$
0=\hat{\mathcal{V}}\left(\frac{4\left|W_{0}\right| \sqrt{\hat{\tau}_{\mathrm{s}}}}{\lambda a|\mathcal{A}|} e^{a \hat{\tau}_{\mathrm{s}}}\right)^{-1}\left(1-4 a \hat{\tau}_{\mathrm{s}}\right)-2\left(1-a \hat{\tau}_{\mathrm{s}}\right)
$$

Employing then 6.44 and observing that in order for our description of nonperturbative effects to be viable we have to require $a \hat{\tau}_{\mathrm{s}} \gg 1$, we find the minimum of the F-term potential (6.42) at values

$$
\left.\hat{\tau}_{\mathrm{s}}\right|_{\text {min. }} \simeq\left(\frac{3 \lambda \hat{\xi}}{16}\right)^{2 / 3},\left.\quad \hat{\mathcal{V}}\right|_{\text {min. }} \simeq \frac{2\left|W_{0}\right| \sqrt{\hat{\tau}_{\mathrm{s}}}}{\lambda a|\mathcal{A}|} e^{a \hat{\tau}_{\mathrm{s}}}
$$

\section{Some Numbers}

Let us finally come back to our explicit example $\mathbb{P}_{[1,1,1,6,9]}[18]$ from the beginning. In this case, we have $\lambda=24 \sqrt{2}$ and the Euler number is $\chi=-540$. Choosing then for instance

$$
a=2 \pi, \quad|\mathcal{A}|=1, \quad\left|W_{0}\right|=10, \quad g_{s}=\frac{3}{4},
$$

and using 3.22 for $\hat{\xi}$, we obtain a minimum of the F-term potential at values (in string frame)

$$
\left.\tau_{\mathrm{s}}\right|_{\min .} \simeq 4.11,\left.\quad \mathcal{V}\right|_{\min .} \simeq 2.56 \times 10^{14}
$$

Here we see that indeed the volume $\mathcal{V}$ of the compactification space is not only large, but exponentially large. In figure 6.3, we have plotted the F-term potential (6.42) for the values 6.47) (and $\varrho=1$ ) confirming our previous analysis. 


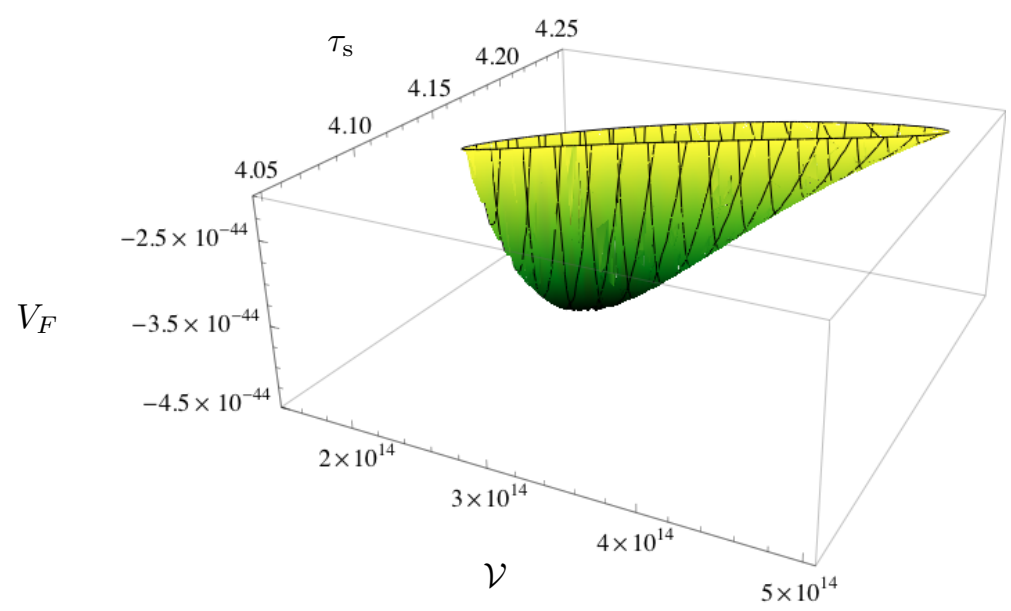

Figure 6.3: Plot of the F-term potential 6.42 for the example $\mathbb{P}_{[1,1,1,6,9]}[18]$ with values (6.47) and $\varrho=1$ in the vicinity of the minimum. This plot agrees with the estimated values 6.48 , and the value of the potential in the minimum is $\left.V_{F}\right|_{\text {min. }} \simeq$ $-4 \times 10^{-44}$.

\subsubsection{Discussion}

To conclude this section, let us now summarise the main features of the LARGE Volume Scenario.

\section{Aspects of Moduli Stabilisation}

- We have seen that in contrast to the KKLT scenario, for the LVS we have a controlled expansion in $1 / \hat{\mathcal{V}}$ which allows us to first stabilise the complex structure moduli and the axio-dilaton, and then to study the stabilisation of Kähler moduli.

- In the LARGE Volume Scenario, we have included $\alpha^{\prime}$-corrections to $\mathcal{K}$ which are crucial for the stabilisation of Kähler moduli. Therefore, our analysis does not rely on the no-scale property of $V_{F}$.

- Note furthermore, in order to find a viable minimum, we did not need to fine-tune the contribution to $W_{0}$ from fluxes. In particular, in our example we have chosen $W_{0}=10$.

- Also, we have explicitly neglected an MSSM sector in our first simple example. However, due to a constraint to be developed in section 8.2 , the moduli stabilisation procedure will be modified when taking into account such a sector. We will study this point in chapter 9 . 
- We have not performed the analysis of the LARGE Volume Scenario for the case $h_{-}^{1,1} \neq 0$ which can however be found in [93].

- Although we have not discussed this point, the uplift from the AdS minimum in the LVS to a de Sitter minimum is usually achieved in the same way as for the KKLT Scenario.

- One of the requirements to obtain a minimum in a LARGE Volume Scenario is the swiss-cheese property $(6.22)$ of the compactification manifold. In the beginning, not many of such manifolds where known but recent work has provided several new examples $[52,72,138]$.

Furthermore, in [139] it has been analysed from a general point of view under which conditions a minimum in a LVS can be obtained. The result is that one essentially needs one blow-up cycle originating from a singularity and one Kähler modulus, or a combination of thereof, which can become very large. In particular, the large combination will be stabilised by the procedure introduced here but each of the components may need a different stabilisation mechanism. Finally, in [139] it was also analysed how Kähler moduli can be stabilised via string loop corrections to the Kähler potential.

- Let us also point out that in the LARGE Volume Scenario the overall volume $\mathcal{V} \leftrightarrow \operatorname{Re}\left(T_{\mathrm{b}}\right)$ of the compactification manifold is stabilised via perturbative effects. However, the corresponding axion $\operatorname{Im}\left(T_{\mathrm{b}}\right)$ does not appear in the Fterm potential and therefore is not stabilised. But, taking into account for instance non-perturbative effects also for the large cycle, this axion will appear in the potential and thus has the chance to be fixed. Although we did not perform an explicit analysis of this point, in the following we will assume that also $\operatorname{Im}\left(T_{\mathrm{b}}\right)$ can be stabilised.

\section{Phenomenological Aspects}

Let us now turn to the phenomenological properties of the LARGE Volume Scenario. Since this work is more concerned with String Theory consistency conditions and mechanisms for moduli stabilisation, we do not discuss this topic in detail. However, we nevertheless would like to make a few comments.

- First, since the LVS minimum does not rely on the vanishing of the Kähler moduli F-terms, in general supersymmetry will be broken. The gravitino mass is given by

$$
m_{3 / 2}=e^{\mathcal{K} / 2}|W| \simeq \frac{\left|W_{0}\right|}{\mathcal{V}} M_{\mathrm{Pl}}
$$


where $M_{\mathrm{Pl}}=2.4 \times 10^{18} \mathrm{GeV}$ is the Planck mass. Note that for our example from above, neglecting numerical prefactors, we find $m_{3 / 2} \sim 100 \mathrm{TeV}$ which indicates that supersymmetry can be broken in a phenomenological viable regime.

In addition, let us consider adding D-branes realising the MSSM to our setup. We are not allowed to wrap MSSM branes on the large cycle with volume $\tau_{\mathrm{b}}$ since this would lead via

$$
\frac{1}{g^{2}} \sim \operatorname{Vol}(\Gamma) \sim \tau
$$

with $\tau$ the volume of the cycle $\Gamma$ wrapped by the D-brane, to an unacceptable small gauge coupling. ${ }^{4}$ On the other hand, placing the MSSM branes on the small cycles $\Gamma_{\tilde{\imath}}$, in our example with $\tau_{\mathrm{s}} \simeq 4.11$, leads to gauge couplings in an phenomenological acceptable regime.

In summary, in the LARGE Volume Scenario it is possible to obtain low-scale supersymmetry breaking and realistic values for the MSSM gauge couplings. For other type II constructions, this is usually much harder to achieve.

- Second, let us mention that the string scale is computed in the following way

$$
m_{\text {string }} \sim \frac{1}{\sqrt{\mathcal{V}}} M_{\mathrm{Pl}}
$$

which for our example from above leads to $m_{\text {string }} \simeq 10^{11} \mathrm{GeV}$. However, by arranging different values for the parameters, one can easily obtain the string scale at the GUT scale, which seems to be disfavoured by a recent finite temperature analysis [141], or for a string scale in the TeV regime.

- Finally, let us mention that due to the exponential large volume of the compactification manifold, some of the physics on the small cycles can be studied from a local perspective [142]. Furthermore, utilising the scalings (6.24), soft supersymmetry breaking terms have been worked out in $[134,57]$ and their collider signatures have been determined in [143].

\footnotetext{
${ }^{4}$ However, in [140] it has been shown that D-branes on the large four-cycle can lead to interesting patterns of global symmetries and hyperweak interactions.
} 


\section{Part III}

\section{Developments}





\section{Chapter 7}

\section{The Generalised Green-Schwarz Mechanism}

In part III of this thesis, we are going to present some of the original work of the author [51-53] which is mainly concerned with the three topics Model Building Constraints for Type IIB Orientifolds with O3- and O7-Planes, D-Brane Instantons, and Moduli Stabilisation in the LARGE Volume Scenario.

In the first chapter of part III, we study the generalised Green-Schwarz mechanism for type IIB orientifolds with O3- and O7-planes. In particular, we derive the tadpole cancellation conditions in this context, we determine the chiral anomalies and check in detail that the latter are cancelled via the Green-Schwarz mechanism, and we compute Fayet-Iliopoulos terms for D7-branes.

\subsection{Tadpole Cancellation Conditions}

In the first section, we will study the tadpole cancellation conditions for orientifolds of type IIB String Theory with O3- and O7-planes. As we will see, in order to satisfy these conditions, we have to introduce D7-branes as well as a combination of D3-branes and background flux.

However, before getting started, let us remark that such systems can also be described via a certain orientifold limit [144] of F-theory [22]. In particular, especially the D3-brane tadpole cancellation condition to be determined below has a corresponding expression in F-theory. 


\subsubsection{Variation of the Action}

\section{Closed String Sector}

From an effective Field Theory point of view, satisfying the tadpole cancellation conditions corresponds to solving (part of) the equations of motion. In order to determine the latter, we recall from (2.11) the effective action for the closed sector of type IIB String Theory in the democratic formulation

$$
\begin{aligned}
\mathcal{S}_{\mathrm{IIB}}=\frac{1}{2 \kappa_{10}^{2}} \int\left[e^{-2 \phi}(\mathrm{R} \star 1+4 d \phi \wedge \star d \phi\right. & \left.-\frac{1}{2} H_{3} \wedge \star H_{3}\right) \\
& \left.-\frac{1}{4} \sum_{p=1,3,5,7,9} \widetilde{F}_{p} \wedge \star \widetilde{F}_{p}\right] .
\end{aligned}
$$

In the following, we will focus on the equation of motion for the Ramond-Ramond fields $C_{8}, C_{6}$ and $C_{4}$ and so we calculate the variation of $(7.1)$ with respect to these fields

$$
\begin{aligned}
& \delta_{C_{4}} \mathcal{S}_{\text {IIB }}=\frac{1}{4 \kappa_{10}^{2}} \int \delta C_{4} \wedge\left(+d \widetilde{F}_{5}-H_{3} \wedge \widetilde{F}_{3}\right), \\
& \delta_{C_{6}} \mathcal{S}_{\text {IIB }}=\frac{1}{4 \kappa_{10}^{2}} \int \delta C_{6} \wedge\left(-d \widetilde{F}_{3}+H_{3} \wedge \widetilde{F}_{1}\right), \\
& \delta_{C_{8}} \mathcal{S}_{\text {IIB }}=\frac{1}{4 \kappa_{10}^{2}} \int \delta C_{8} \wedge\left(+d \widetilde{F}_{1}\right) .
\end{aligned}
$$

Since we only turn on fluxes for $F_{3}$ and $H_{3}$, the term $H_{3} \wedge \widetilde{F}_{1}$ vanishes. For the first line in (7.2), we employ (2.19) and (2.6) to calculate

$$
\frac{1}{l_{s}^{4}} \int_{\mathcal{X}} H_{3} \wedge \widetilde{F}_{3}=\frac{1}{l_{s}^{4}} \int_{\mathcal{X}} \bar{H}_{3} \wedge \bar{F}_{3}=4\left(h_{\hat{\kappa}} f^{\hat{\kappa}}-h^{\hat{\lambda}} f_{\hat{\lambda}}\right)=-4 N_{\text {flux }} \in 4 \mathbb{Z},
$$

where the minus sign has been chosen for later convenience.

\section{Open String Sector}

Let is now turn to the open string sector. The DBI action 2.29) for $\mathrm{D} p$-branes and $\mathrm{O} p$-planes does not depend on the Ramond-Ramond fields $C_{8}, C_{6}$ or $C_{4}$ and so the variation of $\mathcal{S}^{\mathrm{DBI}}$ with respect to the latter vanishes. However, the Chern-Simons action 2.31) for D-branes and O-planes does depend on the R-R potentials $C_{p}$ 
which we recall for convenience

$$
\begin{aligned}
& \mathcal{S}_{\mathrm{D} p}^{\mathrm{CS}}=-\mu_{p} \kappa_{p} \int_{\mathrm{D} p} \operatorname{ch}(\mathcal{F}) \wedge \sqrt{\frac{\hat{\mathcal{A}}\left(\mathcal{R}_{T}\right)}{\hat{\mathcal{A}}\left(\mathcal{R}_{N}\right)}} \wedge \bigoplus_{q} \varphi^{*} C_{q}, \\
& \mathcal{S}_{\mathrm{O} p}^{\mathrm{CS}}=-Q_{p} \mu_{p} \kappa_{p} \int_{\mathrm{O} p} \sqrt{\frac{\mathcal{L}\left(\mathcal{R}_{T} / 4\right)}{\mathcal{L}\left(\mathcal{R}_{N} / 4\right)}} \wedge \bigoplus_{q} \varphi^{*} C_{q} .
\end{aligned}
$$

As we already mentioned, the definition of the Chern character, the $\hat{\mathcal{A}}$-genus and the Hirzebruch polynomial $\mathcal{L}$ can be found in appendix A. In addition, in this appendix we have outlined the calculation leading to the following expressions

$$
\begin{aligned}
& \text { D3 }: \quad \sqrt{\frac{\hat{\mathcal{A}}\left(\mathcal{R}_{T}\right)}{\hat{\mathcal{A}}\left(\mathcal{R}_{N}\right)}}=\left(1+\frac{1}{96}\left(\frac{l_{s}^{2}}{2 \pi}\right)^{2} \operatorname{tr}\left(R^{2}\right)+\ldots\right), \\
& \text { D7 : } \quad \sqrt{\frac{\hat{\mathcal{A}}\left(\mathcal{R}_{T}\right)}{\hat{\mathcal{A}}\left(\mathcal{R}_{N}\right)}}=\left(1+\frac{1}{96}\left(\frac{l_{s}^{2}}{2 \pi}\right)^{2} \operatorname{tr}\left(R^{2}\right)+\ldots\right) \wedge\left(1+\frac{l_{s}^{4}}{24} c_{2}\left(\Gamma_{\mathrm{D} 7}\right)+\ldots\right) \text {, } \\
& \text { O3 }: \quad \sqrt{\frac{\mathcal{L}\left(\mathcal{R}_{T} / 4\right)}{\mathcal{L}\left(\mathcal{R}_{N} / 4\right)}}=\left(1-\frac{1}{192}\left(\frac{l_{s}^{2}}{2 \pi}\right)^{2} \operatorname{tr}\left(R^{2}\right)+\ldots\right) \text {, } \\
& \text { O7 : } \quad \sqrt{\frac{\mathcal{L}\left(\mathcal{R}_{T} / 4\right)}{\mathcal{L}\left(\mathcal{R}_{N} / 4\right)}}=\left(1-\frac{1}{192}\left(\frac{l_{s}^{2}}{2 \pi}\right)^{2} \operatorname{tr}\left(R^{2}\right)+\ldots\right) \wedge\left(1-\frac{l_{s}^{4}}{48} c_{2}\left(\Gamma_{\mathrm{O} 7}\right)+\ldots\right) .
\end{aligned}
$$

Note that $R$ is defined on $\mathbb{R}^{3,1}$ and that the four-form $c_{2}$ is defined on $\mathcal{X}$. Also, we have only shown the terms relevant for the integrals in the Chern-Simons actions.

With the help of 7.5 , we can now compute the variation of the actions (7.4) with respect to $C_{4}, C_{6}$ and $C_{8}$. We find

$$
\begin{aligned}
& \delta_{C_{4}} \mathcal{S}_{\mathrm{D} 3}^{\mathrm{CS}}=+\mu_{3} \int_{\mathbb{R}^{3,1}} \delta C_{4} N_{\mathrm{D} 3}, \\
& \delta_{C_{4}} \mathcal{S}_{\mathrm{O} 3}^{\mathrm{CS}}=+\mu_{3} \int_{\mathbb{R}^{3,1}} \delta C_{4}\left(-\frac{1}{2}\right), \\
& \delta_{C_{4}} \mathcal{S}_{\mathrm{D} 7}^{\mathrm{CS}}=-\mu_{7} \int_{\mathbb{R}^{3,1}} \delta C_{4} \wedge \int_{\Gamma_{\mathrm{D} 7}}\left(\operatorname{ch}_{2}(\overline{\mathcal{F}})+l_{s}^{4} N_{\mathrm{D} 7} \frac{c_{2}\left(\Gamma_{\mathrm{D} 7}\right)}{24}\right), \\
& \delta_{C_{4}} \mathcal{S}_{\mathrm{O} 7}^{\mathrm{CS}}=-\mu_{7} \int_{\mathbb{R}^{3,1}} \delta C_{4} \wedge \int_{\Gamma_{\mathrm{O} 7}} l_{s}^{4} \frac{c_{2}\left(\Gamma_{\mathrm{O} 7}\right)}{6},
\end{aligned}
$$

with $N_{\mathrm{D} 3}=\operatorname{ch}_{0}\left(\mathcal{F}_{\mathrm{D} 3}\right)$ and $N_{\mathrm{D} 7}=\operatorname{ch}_{0}\left(\mathcal{F}_{\mathrm{D} 7}\right)$ denoting the number of D3- respectively D7-branes on top of each other. Furthermore, $\Gamma$ is again the holomorphic four-cycle wrapped by the D7-branes and O7-planes in the compact space, and $\overline{\mathcal{F}}$ 
stands for the part of $\mathcal{F}$ in $\mathcal{X}$. In a similar way as above, we compute the variation of the Chern-Simons actions with respect to $C_{6}$ as follows

$$
\delta_{C_{6}} \mathcal{S}_{\mathrm{D} 7}^{\mathrm{CS}}=-\mu_{7} \int_{\mathbb{R}^{3,1} \times \Gamma_{\mathrm{D} 7}}\left(\varphi^{*} \delta C_{6}\right) \wedge \operatorname{ch}_{1}(\overline{\mathcal{F}}), \quad \delta_{C_{6}} \mathcal{S}_{\mathrm{O} 7}^{\mathrm{CS}}=0,
$$

and the variation with respect to $C_{8}$ is found to be

$$
\delta_{C_{8}} \mathcal{S}_{\mathrm{D} 7}^{\mathrm{CS}}=-\mu_{7} \int_{\mathbb{R}^{3,1} \times \Gamma_{\mathrm{D} 7}}\left(\varphi^{*} \delta C_{8}\right) N_{\mathrm{D} 7}, \quad \delta_{C_{8}} \mathcal{S}_{\mathrm{O} 7}^{\mathrm{CS}}=-\mu_{7} \int_{\mathbb{R}^{3,1} \times \Gamma_{\mathrm{O} 7}}\left(\varphi^{*} \delta C_{8}\right)(-8)
$$

\subsubsection{Tadpole Cancellation Conditions}

Combining the results from the previous subsection, we can now determine the tadpole cancellation conditions for type IIB orientifolds with D3- and D7-branes. However, due to the orientifold projection $\Omega(-1)^{F_{L}} \sigma$, we have to take into account the orientifold planes as well as the orientifold images of the D-branes. Denoting these images by a prime, the schematic form of the full action reads

$$
\mathcal{S}=\frac{1}{2}\left(\mathcal{S}_{\mathrm{IIB}}+\mathcal{S}_{\mathrm{IIB}}^{\prime}+\sum_{a, a^{\prime}} \mathcal{S}_{\mathrm{D} 7_{a}}^{\mathrm{CS}}+\sum_{i} \mathcal{S}_{\mathrm{O} 7_{i}}^{\mathrm{CS}}+\sum_{b, b^{\prime}} \mathcal{S}_{\mathrm{D} 3_{b}}^{\mathrm{CS}}+\sum_{j} \mathcal{S}_{\mathrm{O}_{j}}^{\mathrm{CS}}\right)
$$

In order to be more concrete later, using $(2.12)$ and (2.27), we determine the data for an orientifold image of a $\mathrm{D} p$-brane as follows

$$
\begin{aligned}
& \Gamma_{\mathrm{D} p}^{\prime}=\Omega(-1)^{F_{L}} \sigma \Gamma_{\mathrm{D} p}=(-1)^{\frac{p+1}{2}} \sigma \Gamma_{\mathrm{D} p}, \\
& \overline{\mathcal{F}}^{+^{\prime}}=\Omega(-1)^{F_{L}} \sigma^{*} \overline{\mathcal{F}}^{+}=-\sigma^{*} \overline{\mathcal{F}}^{+},
\end{aligned}
$$

where in particular $\Gamma_{\mathrm{D} 7}$ is a divisor in $\mathcal{X}$ while $\Gamma_{\mathrm{D} 3}$ is a point in $\mathcal{X}$ corresponding to a D3-brane.

\section{D7-Brane Tadpole Cancellation Condition}

The equations of motion for $C_{8}$ are obtained by setting to zero the variation of (7.9) with respect to $C_{8}$. Using (7.2) and (7.8), we compute

$$
0=\frac{1}{2} \frac{2 \pi}{l_{s}^{8}} \int_{\mathbb{R}^{3,1} \times \mathcal{X}} \delta C_{8} \wedge\left[d \widetilde{F}_{1}-\sum_{\mathrm{D} 7_{a}, \mathrm{D} 7_{a^{\prime}}} N_{\mathrm{D} 7_{a}}\left[\Gamma_{\mathrm{D} 7_{a}}\right]-\sum_{\mathrm{O} 7_{i}}(-8)\left[\Gamma_{\mathrm{O} 7_{i}}\right]\right],
$$


where $N_{\mathrm{D} 7_{a}}$ is the total number of D7-branes with gauge flux $\bar{F}_{a}$ wrapping the fourcycle $\Gamma_{\mathrm{D} 7_{a}}$, and $[\Gamma]$ stands for the Poincaré dual of the four-cycle $\Gamma$ in $\mathcal{X}$. Since the variations $\delta C_{8}$ are arbitrary and $d \widetilde{F}_{1}$ is exact, in co-homology the expression above can be written as

$$
\sum_{\mathrm{D} 7_{a}} N_{\mathrm{D} 7_{a}}\left(\left[\Gamma_{\mathrm{D} 7_{a}}\right]+\left[\Gamma_{\mathrm{D} 7_{a}}^{\prime}\right]\right)=8 \sum_{\mathrm{O} 7_{i}}\left[\Gamma_{\mathrm{O} 7_{i}}\right]
$$

which is known as the D7-brane tadpole cancellation condition. Note that from this equation we see that if there are O7-planes present, we are forced to introduce D7-branes into our setup.

\section{D5-Brane Tadpole Cancellation Condition}

Employing (7.2 and 7.7 as well as the basis of (1,1)-forms $\left\{\omega_{I}\right\} \in H_{\bar{\partial}}^{1,1}(\mathcal{X})$ introduced in table 2.1, the equations of motion originating from $C_{6}$ are found to be of the following form

$$
\begin{aligned}
0 & =\sum_{\mathrm{D} 7_{a}}\left(\int_{\Gamma_{\mathrm{D} 7_{a}}} \operatorname{ch}_{1}\left(\overline{\mathcal{F}}_{a}\right) \wedge \omega_{I}+\int_{\Gamma_{\mathrm{D} 7_{a}}^{\prime}} \operatorname{ch}_{1}\left(\overline{\mathcal{F}}_{a}^{\prime}\right) \wedge \omega_{I}\right) \\
& =\sum_{\mathrm{D} 7_{a}} \int_{\mathcal{X}}\left(\operatorname{ch}_{1}\left(\varphi_{*} \overline{\mathcal{F}}_{a}\right) \wedge\left[\Gamma_{\mathrm{D} 7_{a}}\right]+\operatorname{ch}_{1}\left(\varphi_{*} \overline{\mathcal{F}}_{a}^{\prime}\right) \wedge\left[\Gamma_{\mathrm{D} 7_{a}}^{\prime}\right]\right) \wedge \omega_{I}
\end{aligned}
$$

where the prime denotes again the $\Omega(-1)^{F_{L}} \sigma$ image and $\varphi_{*} \overline{\mathcal{F}}$ is the push-forward of $\overline{\mathcal{F}}$ from the D7-brane to the Calabi-Yau manifold $\mathcal{X}$.

Note that 7.13 is not trivially vanishing which can be seen by utilising the relation

$$
\int_{\mathcal{X}} \sigma^{*} \omega_{I} \wedge \sigma^{*} \omega_{J} \wedge \sigma^{*} \omega_{K}=\int_{\mathcal{X}} \omega_{I} \wedge \omega_{J} \wedge \omega_{K}
$$

In particular, recalling from 2.27 that $\mathcal{F}$ is odd under $\Omega(-1)^{F_{L}}$, we can rewrite equation (7.13) as

$$
0=\sum_{\mathrm{D} 7_{a}} \operatorname{ch}_{1}\left(\varphi_{*} \overline{\mathcal{F}}_{a}\right) \wedge\left[\Gamma_{\mathrm{D} 7_{a}}\right] \wedge\left(\omega_{I}-\sigma^{*} \omega_{I}\right)
$$

which is a non-trivial constraint if $h_{-}^{1,1} \neq 0$.

However, the D5-brane tadpole cancellation condition is not yet satisfying (here we follow [72]). In order to explain this point, let us recall from our discussion 
below equation 2.13 that $\overline{\mathcal{F}}$ contains $B_{2}^{-}$which takes continuous values. Since the tadpole cancellation conditions usually involve only discrete quantities, the dependence on $B_{2}^{-}$should disappear. And indeed, using the definition of the Chern character $(\mathrm{A} .1)$ as well as $(2.13)$ and $(2.25)$, we compute

$$
\operatorname{ch}_{1}\left(\varphi_{*} \overline{\mathcal{F}}_{a}\right)=\operatorname{ch}_{1}\left(\varphi_{*} \overline{\mathcal{F}}_{a}^{+}\right)+N_{\mathrm{D} 7} B_{2}^{-}
$$

Employing then the D7-brane tadpole cancellation condition 7.12 , we find for the $B_{2}^{-}$terms in 7.13 that

$$
\begin{aligned}
\sum_{\mathrm{D} 7_{a}} \int_{\mathcal{X}}\left(N_{\mathrm{D} 7_{a}} B_{2}^{-}\right. & \left.\wedge\left[\Gamma_{\mathrm{D} 7_{a}}\right]+N_{\mathrm{D} 7_{a}} B_{2}^{-} \wedge\left[\Gamma_{\mathrm{D} 7_{a}}^{\prime}\right]\right) \wedge \omega_{I} \\
& =8 \sum_{\mathrm{O} 7_{i}} \int_{\mathcal{X}}\left[\Gamma_{\mathrm{O} 7_{i}}\right] \wedge B_{2}^{-} \wedge \omega_{I}=8 \sum_{\mathrm{O}_{i}} \int_{\Gamma_{\mathrm{O} 7_{i}}} \varphi^{*} B_{2}^{-} \wedge \omega_{I} .
\end{aligned}
$$

The final step is to observe that since the orientifold planes are pointwise invariant under the involution $\sigma$, there are no odd two-cycles on $\Gamma_{\mathrm{O} 7}$, that is $H_{2-}\left(\Gamma_{\mathrm{O} 7}, \mathbb{Z}\right)=0$. Because $B_{2}^{-}$is in $H_{\bar{\partial}}^{1,1}(\mathcal{X})$, we see that in this case $\varphi^{*} B_{2}^{-}=0$ and so the integral (7.17) vanishes. The D5-brane tadpole cancellation condition therefore contains only discrete quantities and reads

$$
0=\sum_{\mathrm{D} 7_{a}}\left(\operatorname{ch}_{1}\left(\varphi_{*} \overline{\mathcal{F}}_{a}^{+}\right) \wedge\left[\Gamma_{\mathrm{D} 7_{a}}\right]+\operatorname{ch}_{1}\left(\varphi_{*} \overline{\mathcal{F}}_{a}^{+^{\prime}}\right) \wedge\left[\Gamma_{\mathrm{D} 7_{a}}^{\prime}\right]\right) \wedge \omega_{I}
$$

\section{D3-Brane Tadpole Cancellation Condition}

Let us finally study the equation of motion for $C_{4}$ which is obtained by setting to zero the variation of (7.9) with respect to $C_{4}$. Employing (7.2) and (7.6), we compute

$$
\begin{aligned}
0=\frac{1}{2} & \frac{2 \pi}{l_{s}^{4}} \int_{\mathbb{R}^{3,1}} \delta C_{4} \wedge\left[\frac{1}{l_{s}^{4}} \int_{\mathcal{X}}\left(d \widetilde{F}_{5}-H_{3} \wedge \widetilde{F}_{3}\right)+\sum_{\mathrm{D} 3_{b}, \mathrm{D} 3_{b^{\prime}}} N_{\mathrm{D} 3_{b}}-\sum_{\mathrm{O} 7_{i}} \frac{N_{\mathrm{O}_{i}}}{2}\right. \\
& \left.-\frac{1}{l_{s}^{4}} \sum_{\mathrm{D} 7_{a}, \mathrm{D} 7_{a^{\prime}}} \int_{\Gamma_{\mathrm{D} 7_{a}}}\left(\operatorname{ch}_{2}\left(\overline{\mathcal{F}}_{a}\right)+l_{s}^{4} N_{\mathrm{D} 7_{a}} \frac{c_{2}\left(\Gamma_{\mathrm{D}_{a}}\right)}{24}\right)-\sum_{\mathrm{O} 7_{j}} \int_{\Gamma_{\mathrm{O} 7_{j}}} \frac{c_{2}\left(\Gamma_{\mathrm{O} 7_{j}}\right)}{6}\right] .
\end{aligned}
$$

By the same arguments as for the D5-brane tadpole, the dependence of (7.19) on $B_{2}^{-}$should vanish. In order to see this, we employ the definition A.1 to obtain

$$
\operatorname{ch}_{2}(\overline{\mathcal{F}})=\operatorname{ch}_{2}\left(\overline{\mathcal{F}}^{+}\right)+\operatorname{ch}_{1}\left(\overline{\mathcal{F}}^{+}\right) \wedge\left(\varphi^{*} B_{2}^{-}\right)+\frac{N_{\mathrm{D} 7}}{2}\left(\varphi^{*} B_{2}^{-}\right)^{2},
$$


which we use to calculate

$$
\begin{aligned}
& \sum_{\mathrm{D} 7_{a}, \mathrm{D} 7_{a^{\prime}}} \int_{\Gamma_{\mathrm{D} 7 a}} \operatorname{ch}_{2}\left(\overline{\mathcal{F}}_{a}\right) \\
= & \sum_{\mathrm{D} 7_{a}, \mathrm{D} 7_{a^{\prime}}} \int_{\Gamma_{\mathrm{D} 7 a}}\left[\operatorname{ch}_{2}\left(\overline{\mathcal{F}}_{a}^{+}\right)+\operatorname{ch}_{1}\left(\overline{\mathcal{F}}_{a}^{+}\right) \wedge\left(\varphi^{*} B_{2}^{-}\right)\right] \\
& \quad+\frac{1}{2} \sum_{\mathrm{D} 7_{a}} \int_{\mathcal{X}}\left(B_{2}^{-}\right)^{2} N_{\mathrm{D} 7_{a}}\left(\left[\Gamma_{\mathrm{D} 7_{a}}\right]+\left[\Gamma_{\mathrm{D} 7_{a}}^{\prime}\right]\right) \\
= & \sum_{\mathrm{D} 7_{a}, \mathrm{D} 7_{a^{\prime}}} \int_{\Gamma_{\mathrm{D} 7 a}} \operatorname{ch}_{2}\left(\overline{\mathcal{F}}_{a}^{+}\right) .
\end{aligned}
$$

In going from the second to the third line, we utilised the D5-brane tadpole cancellation condition to observe that the terms involving $\operatorname{ch}_{1}\left(\overline{\mathcal{F}}^{+}\right)$have to vanish, and for the cancellation of the expressions containing $\left(B_{2}^{-}\right)^{2}$, we used the same reasoning as in (7.17).

Next, following [145] (see also [146]), D7-branes on the orientifold space can have double-intersection points and can therefore be singular. 11 Thus, the definition of the corresponding Euler characteristic

$$
\chi(\Gamma)=\int_{\Gamma} c_{2}(\Gamma)
$$

is ambiguous. However, as has been explained in [147,145], the Euler characteristic of an appropriate blow-up $\Sigma$ of the singularity minus the number $n_{\mathrm{pp}}$ of pinchpoints,

$$
\chi_{o}(\Gamma)=\chi(\Sigma)-n_{\mathrm{pp}},
$$

leads to the correct result. We will denote the physical Euler characteristic of $[147$, $145]$ by $\chi_{o}(\Gamma)$, which reduces to the usual Euler characteristic 7.22 for smooth D7-branes.

Employing equation 7.3 for the background fluxes and denoting the total number of D3-branes by $N_{\mathrm{D} 3}$ as well as the total number of O3-planes by $N_{\mathrm{O} 3}$, we deduce from $(7.19)$ the D3-brane tadpole cancellation condition to be of the form

$$
N_{\mathrm{D} 3}+2 N_{\text {flux }}=\frac{N_{\mathrm{O} 3}}{4}+\frac{1}{l_{s}^{4}} \sum_{\mathrm{D} 7_{a}} \int_{\Gamma_{\mathrm{D} 7_{a}}} \operatorname{ch}_{2}\left(\overline{\mathcal{F}}_{a}^{+}\right)+\sum_{\mathrm{D} 7_{a}} N_{\mathrm{D} 7_{a}} \frac{\chi_{o}\left(\Gamma_{\mathrm{D} 7_{a}}\right)}{24}+\sum_{\mathrm{O} 7_{j}} \frac{\chi\left(\Gamma_{\mathrm{O} 7_{j}}\right)}{12} .
$$

\footnotetext{
${ }^{1}$ An example for such a singularity is the complexified Whitney umbrella given by the equation $x^{2}=z y^{2}$ with $x, y, z \in \mathbb{C}[145]$.
} 
Note that in general, in order to satisfy this equation we have to introduce a combination D3-branes and background flux.

\section{Relation to F-Theory}

To conclude this section, let us illustrate how the D3-brane tadpole cancellation condition is related to F-theory. In particular, we see that by placing four D7branes without gauge flux on top of a single O7-plane, equation (7.24 becomes

$$
N_{\mathrm{D} 3}+2 N_{\text {flux }}=\frac{N_{\mathrm{O} 3}}{4}+\frac{\chi\left(\Gamma_{\mathrm{O} 7}\right)}{4}
$$

For this solution, the lift to F-theory is given by the Calabi-Yau four-fold $\mathcal{Y}=$ $\left(\mathcal{X} \times \mathbb{T}^{2}\right) / \mathbb{Z}_{2}$ where the $\mathbb{Z}_{2}$ action is realised by the holomorphic involution $\sigma$ on the Calabi-Yau three-fold $\mathcal{X}$ together with the action $z \rightarrow-z$ on the two-torus. Blowing up the four $\mathbb{Z}_{2}$ singularities originating from the fixed points of $\mathbb{Z}_{2}$ on $\mathbb{T}^{2}$ and gluing in $\mathbb{P}^{1} \mathrm{~s}$, the Euler characteristic of $\mathcal{Y}$ is computed as

$$
\begin{aligned}
\chi(\mathcal{Y}) & =\frac{1}{2}\left(\chi\left(\mathcal{X} \times \mathbb{T}^{2}\right)-4 \chi\left(\Gamma_{\mathrm{O} 7}\right)-4 N_{\mathrm{O} 3}\right)+4 \chi\left(\mathbb{P}^{1}\right) \chi\left(\Gamma_{\mathrm{O} 7}\right)+4 \chi\left(\mathbb{P}^{1}\right) N_{\mathrm{O} 3} \\
& =-2 \chi\left(\Gamma_{\mathrm{O} 7}\right)-2 N_{\mathrm{O} 3}+8 \chi\left(\Gamma_{\mathrm{O} 7}\right)+8 N_{\mathrm{O} 3} \\
& =24\left(\frac{N_{\mathrm{O} 3}}{4}+\frac{\chi\left(\Gamma_{\mathrm{O} 7}\right)}{4}\right),
\end{aligned}
$$

where we used that $\chi\left(\mathbb{T}^{2}\right)=0$ as well as that $\chi\left(\mathbb{P}^{1}\right)=2$. Employing this result we see that, in agreement with the F-theory result [148], we can write equation (7.25) as

$$
N_{\text {D3 }}+2 N_{\text {flux }}=\frac{\chi(\mathcal{Y})}{24} .
$$

\subsection{The Generalised Green-Schwarz Mechanism}

In this section, we are first going to determine the chiral anomalies arising in a general configuration of intersecting D7-branes with gauge flux, and then explicitly check that via the generalised Green-Schwarz mechanism indeed the chiral anomalies are cancelled. 


\subsubsection{Chiral Anomalies}

The anomaly coefficients for the chiral anomalies are expressed in terms of the cubic Casimir $A(r)$, the index $C(r)$ and the $U(1)$ charge $Q(r)$ where $r$ denotes a particular representation. For $S U(N)$, these quantities are summarised in table 7.1 and the discussion for $S O(2 N)$ and $S p(2 N)$ gauge groups can be found in appendix D. More concretely, the cubic non-abelian, the mixed abelian-non-abelian, the cubic abelian and the mixed abelian-gravitational anomalies are calculated via the following formulas (see for instance [149])

$$
\begin{aligned}
\mathcal{A}_{S U\left(N_{\mathrm{D} 7_{a}}\right)^{3}} & =\sum_{r} A(r), \\
\mathcal{A}_{U(1)_{a}-S U\left(N_{\mathrm{D} 7_{b}}\right)^{2}} & =\sum_{r} Q_{a}(r) C_{b}(r), \\
\mathcal{A}_{U(1)_{a}-U(1)_{b}^{2}} & =\sum_{r} Q_{a}(r) Q_{b}^{2}(r) \operatorname{dim}(r), \\
\mathcal{A}_{U(1)_{a}-G^{2}} & =\sum_{r} Q_{a}(r) \operatorname{dim}(r) .
\end{aligned}
$$

\section{Cubic Non-Abelian Anomaly}

For the computation of the cubic non-abelian anomaly, we focus on the D7-brane labelled by $a$ and calculate using (4.3)

$$
\begin{aligned}
\mathcal{A}_{S U\left(N_{\mathrm{D} 7 a}\right)^{3}} & =\sum_{\mathrm{D} 7_{b}} N_{\mathrm{D} 7_{b}}\left(I_{b a}+I_{b^{\prime} a}\right)-8 \sum_{\mathrm{O} 7_{i}} I_{\mathrm{O} 7_{i} a} \\
= & -\frac{1}{l_{s}^{6}} \int_{\mathcal{X}} \frac{\operatorname{ch}_{1}\left(\varphi_{*} \overline{\mathcal{F}}_{a}^{+}\right)}{N_{\mathrm{D} 7_{a}}} \wedge\left[\Gamma_{\mathrm{D} 7_{a}}\right] \wedge\left(\sum_{\mathrm{D} 7_{b}} N_{\mathrm{D} 7_{b}}\left(\left[\Gamma_{\mathrm{D} 7_{b}}\right]+\left[\Gamma_{\mathrm{D} 7_{b}}^{\prime}\right]\right)-8 \sum_{\mathrm{O} 7_{i}}\left[\Gamma_{\mathrm{O} 7_{i}}\right]\right) \\
& +\frac{1}{l_{s}^{6}} \int_{\mathcal{X}} \sum_{\mathrm{D} 7_{b}}\left(\operatorname{ch}_{1}\left(\varphi_{*} \overline{\mathcal{F}}_{b}^{+}\right) \wedge\left[\Gamma_{\mathrm{D} 7_{b}}\right]+\operatorname{ch}_{1}\left(\varphi_{*} \overline{\mathcal{F}}_{b}^{+\prime}\right) \wedge\left[\Gamma_{\mathrm{D} 7_{b}}^{\prime}\right]\right) \wedge\left[\Gamma_{\mathrm{D} 7_{a}}\right] .
\end{aligned}
$$

Here, the prime again denotes the $\Omega(-1)^{F_{L}} \sigma$ image and the sums run over all D7branes $b$ respectively all O7-planes. Employing the D7-brane tadpole cancellation condition (7.12), we see that the first line in (7.29) vanishes. For the vanishing of the second line, we use the D5-brane tadpole cancellation condition $(7.18)$ to arrive at

$$
\mathcal{A}_{S U\left(N_{\mathrm{D} 7 a}\right)^{3}}=0
$$




\begin{tabular}{c||c|c|c|c|c|c} 
& $F$ & $\bar{F}$ & $S$ & $\bar{S}$ & $A$ & $\bar{A}$ \\
\hline \hline $\operatorname{dim}(r)$ & $N$ & $N$ & $\frac{N(N+1)}{2}$ & $\frac{N(N+1)}{2}$ & $\frac{N(N-1)}{2}$ & $\frac{N(N-1)}{2}$ \\
$Q(r)$ & +1 & -1 & +2 & -2 & +2 & -2 \\
$C(r)$ & $\frac{1}{2}$ & $\frac{1}{2}$ & $\frac{N+2}{2}$ & $\frac{N+2}{2}$ & $\frac{N-2}{2}$ & $\frac{N-2}{2}$ \\
$A(r)$ & +1 & -1 & $N+4$ & $-(N+4)$ & $N-4$ & $-(N-4)$
\end{tabular}

Table 7.1: Group theoretical quantities for $S U(N)$ where $F$ stands for the fundamental, $S$ for the symmetric and $A$ for the anti-symmetric representation (see for instance [149]).

\section{Mixed Abelian-Non-Abelian Anomaly}

Next, we consider the mixed abelian-non-abelian anomaly. Along the same lines as above, we compute

$$
\begin{aligned}
\mathcal{A}_{U(1)_{a}-S U\left(N_{\mathrm{D} 7_{b}}\right)^{2}} & =\frac{1}{2} \delta_{a b}\left(\sum_{\mathrm{D} 7_{c}} N_{\mathrm{D} 7_{c}}\left(I_{c b}+I_{c^{\prime} b}\right)-8 \sum_{\mathrm{O} 7_{i}} I_{\mathrm{O} 7_{i} b}\right)-\frac{N_{\mathrm{D} 7_{a}}}{2}\left(I_{a b}-I_{a^{\prime} b}\right) \\
& =-\frac{N_{\mathrm{D} 7_{a}}}{2}\left(I_{a b}-I_{a^{\prime} b}\right)
\end{aligned}
$$

where we used, similarly as for the cubic non-abelian anomaly, the tadpole cancellation conditions 7.12 and $(7.18)$ for the vanishing of the first term.

\section{Cubic Abelian Anomaly}

For the cubic abelian anomaly, we find

$$
\begin{aligned}
& \mathcal{A}_{U(1)_{a}-U(1)_{b}^{2}} \\
& \quad=\frac{N_{\mathrm{D} 7_{a}}}{3} \delta_{a b}\left(\sum_{\mathrm{D} 7_{c}} N_{\mathrm{D} 7_{c}}\left(I_{c b}+I_{c^{\prime} b}\right)-8 \sum_{\mathrm{O} 7_{i}} I_{\mathrm{O} 7_{i} b}\right)-N_{\mathrm{D} 7_{a}} N_{\mathrm{D} 7_{b}}\left(I_{a b}-I_{a^{\prime} b}\right) \\
& \quad=-N_{\mathrm{D} 7_{a}} N_{\mathrm{D} 7_{b}}\left(I_{a b}-I_{a^{\prime} b}\right)
\end{aligned}
$$

where the pre-factor $\frac{1}{3}$ is due to the additional symmetry in the case $a=b$, and we again used the tadpole cancellation conditions (7.12) and (7.18). 


\section{Mixed Abelian-Gravitational Anomaly}

From (7.28), we finally determine the mixed abelian-gravitational anomaly of a D7-brane $a$. Employing the tadpole cancellation condition (7.12), we obtain

$$
\begin{aligned}
\mathcal{A}_{U(1)_{a}-G^{2}} & =N_{\mathrm{D} 7_{a}}\left(\sum_{\mathrm{D} 7_{b}} N_{\mathrm{D} 7_{b}}\left(I_{b a}+I_{b^{\prime} a}\right)-2 \sum_{\mathrm{O} 7_{i}} I_{\mathrm{O}_{i} a}\right) \\
& =N_{\mathrm{D} 7_{a}}\left(\sum_{\mathrm{D} 7_{b}} N_{\mathrm{D} 7_{b}}\left(I_{b a}+I_{b^{\prime} a}\right)-8 \sum_{\mathrm{O} 7_{i}} I_{\mathrm{O}_{i} a}\right)+6 N_{\mathrm{D}_{a}} \sum_{\mathrm{O} 7_{i}} I_{\mathrm{O}_{i} a} \\
& =6 N_{\mathrm{D} 7_{a}} \sum_{\mathrm{O}_{i}} I_{\mathrm{O} 7_{i} a} .
\end{aligned}
$$

\subsubsection{Green-Schwarz Couplings}

After having determined the chiral anomalies in a general configuration of intersecting D7-branes with gauge flux, let us now compute the couplings in the four-dimensional theory which, as explained in section 4.3, are used to cancel the anomalies via the generalised Green-Schwarz mechanism.

\section{Dimensional Reduction of R-R Potentials}

In particular, in the present context the two-forms $A_{(2)}$ and scalars $B_{(0)}$ introduced above equation (4.4) are obtained from a dimensional reduction of the $\mathrm{R}$ - $\mathrm{R} p$-form potentials $C_{p}$ and the duality (4.4) is provided by (2.10). To see this in more detail, we perform a dimensional reduction of $C_{2}, C_{4}$ and $C_{6}$ on the Calabi-Yau manifold $\mathcal{X}$

$$
\begin{aligned}
& C_{2}=\mathcal{C}^{I} \omega_{I}+\mathcal{D}_{0} \\
& C_{4}=C_{I} \sigma^{I}+D^{I} \wedge \omega_{I}+\ldots \\
& C_{6}=
\end{aligned}
$$

where $C_{I}$ respectively $\mathcal{C}^{I}$ are four-dimensional scalars and $D^{I}$ as well as $\mathcal{D}_{0}, \mathcal{D}_{I}$ are two-forms in $\mathbb{R}^{3,1}$. The ellipsis indicate that there are further terms coming for instance from the reduction of $C_{p}$ on three-cycles and from the reduction on $\mathcal{X}$. However, these terms will not be of relevance here. Let us also note that from (2.10, we obtain

$$
\begin{array}{lll}
d \mathcal{C}^{I}=-\star_{4} d \mathcal{D}_{I} & \Rightarrow & \mathcal{C}^{I} \leftrightarrow-\mathcal{D}_{I} \\
d C_{I}=+\star_{4} d D^{I} & \Rightarrow & C_{I} \leftrightarrow+D^{I}
\end{array}
$$


where $\star_{4}$ is the Hodge- $\star$-operator in four dimensions. The relative sign between these two dualities will be important in the following.

\section{Some Technical Details}

We now turn to the couplings (4.5) which are contained in the Chern-Simons actions of the D-branes and O-planes. To determine these, we expand the divisor wrapped by a D7-brane $a$ as

$$
\Gamma_{\mathrm{D} 7_{a}}=m_{a}^{I} \gamma_{I}, \quad m_{a}^{I} \in \mathbb{Z},
$$

where $\left\{\gamma_{I}\right\}$ is the basis of four-cycles introduced in table 2.1. Next, since here we are considering gauge groups $U(N)$ for which the corresponding algebra satisfies $\mathfrak{u}(N) \simeq \mathfrak{u}(1) \times \mathfrak{s u}(N)$, we write the four-dimensional open string field strength $F$ as

$$
F=f \mathbb{1}+\sum_{A} \mathrm{~F}^{A} T^{A}
$$

where $f$ denotes the abelian and $\mathrm{F}^{A}$ stands for the non-abelian part. For the antisymmetric representations matrices $T^{A}$ of the gauge group in the fundamental representation, we have

$$
\operatorname{tr}\left(T^{A}\right)=0, \quad \operatorname{tr}\left(T^{A} T^{B}\right)=\frac{1}{2} \delta^{A B},
$$

where the latter relation reflects the usual choice of normalisation. Concerning the gauge flux $\bar{F}$ on the D7-branes, we make the simplifying assumption to only consider $U(1)$ gauge fluxes on the D7-branes which are diagonally embedded into $U\left(N_{\mathrm{D} 7}\right)$ in the following way

$$
\bar{F}=\bar{f} \mathbb{1}_{N_{\mathrm{D} 7} \times N_{\mathrm{D} 7}} .
$$

Let us emphasise that our forthcoming discussion relies on this choice of flux and its embedding. For a different structure group $\bar{H}=U(1)$ or embedding into $G=U\left(N_{\mathrm{D} 7}\right)$, the calculations become slightly more involved.

Then, using (7.39) and (7.37) together with (7.38), we can evaluate some quantities needed in the following

$$
\begin{aligned}
\operatorname{ch}_{1}(\mathcal{F}) & =N\left(l_{s}^{2} \frac{f+\bar{f}}{2 \pi}+\varphi^{*} B_{2}\right), \\
\operatorname{ch}_{2}(\mathcal{F}) & =\frac{1}{2}\left[\frac{l_{s}^{4}}{8 \pi^{2}} \sum_{A} \mathrm{~F}^{A} \mathrm{~F}^{A}+N\left(l_{s}^{2} \frac{f+\bar{f}}{2 \pi}+\varphi^{*} B_{2}\right)^{2}\right], \\
\operatorname{ch}_{3}(\mathcal{F}) & =\frac{1}{6}\left[\frac{3 l_{s}^{4}}{8 \pi^{2}} \sum_{A} \mathrm{~F}^{A} \mathrm{~F}^{A}\left(l_{s}^{2} \frac{\bar{f}}{2 \pi}+\varphi^{*} B_{2}\right)+N\left(l_{s}^{2} \frac{f+\bar{f}}{2 \pi}+\varphi^{*} B_{2}\right)^{3}\right] .
\end{aligned}
$$




\section{Determining the Green-Schwarz Couplings}

Given these expressions, we can now identify the Green-Schwarz couplings. In particular, the $\operatorname{tr}(F)$ terms are obtained from the D7-brane action and read

$$
\begin{aligned}
\mathcal{S}_{\mathrm{D} 7}^{\mathrm{CS}} & =-\mu_{7} \kappa_{7} \int_{\mathrm{D} 7}\left[\operatorname{ch}_{1}(\mathcal{F}) \wedge C_{6}+\operatorname{ch}_{2}(\mathcal{F}) \wedge C_{4}\right]+\ldots \\
& =-\frac{2 \pi}{l_{s}^{4}} \int_{\mathbb{R}^{3,1}} \frac{l_{s}^{2}}{2 \pi} N_{\mathrm{D} 7} f \wedge\left[\mathcal{D}_{I} m^{I}+\frac{1}{l_{s}^{4}} D^{I} \wedge \int_{\Gamma_{\mathrm{D} 7}}\left(\frac{l_{s}^{2}}{2 \pi} \bar{f}+\varphi^{*} B_{2}^{+}\right) \wedge \omega_{I}\right]+\ldots
\end{aligned}
$$

where the ellipsis denote further couplings not of importance here. The relevant terms involving $\operatorname{tr}\left(F^{2}\right)$ read

$$
\begin{aligned}
\mathcal{S}_{\mathrm{D} 7}^{\mathrm{CS}}=-\mu_{7} \kappa_{7} \int_{\mathrm{D} 7}\left[\operatorname{ch}_{2}(\mathcal{F})\right. & \left.\wedge C_{4}+\operatorname{ch}_{3}(\mathcal{F}) \wedge C_{2}\right]+\ldots \\
=-\frac{2 \pi}{l_{s}^{4}} \int_{\mathbb{R}^{3,1}} \frac{1}{2}\left(\frac{l_{s}^{2}}{2 \pi}\right)^{2} & \left(\frac{1}{2} \sum_{A} \mathrm{~F}^{A} \mathrm{~F}^{A}+N_{\mathrm{D} 7} f^{2}\right) \wedge \\
& \wedge\left[C_{I} m^{I}+\frac{1}{l_{s}^{4}} \mathcal{C}^{I} \int_{\Gamma_{\mathrm{D} 7}}\left(\frac{l_{s}^{2}}{2 \pi} \bar{f}+\varphi^{*} B_{2}^{+}\right) \wedge \omega_{I}\right]+\ldots
\end{aligned}
$$

The $\operatorname{tr}\left(R^{2}\right)$ couplings are contained in the D7-brane action and can be determined using (7.5) to be of the following form

$$
\begin{aligned}
\mathcal{S}_{\mathrm{D} 7}^{\mathrm{CS}}=-\mu_{7} \kappa_{7} \int_{\mathrm{D} 7} \frac{1}{96}\left(\frac{l_{s}^{2}}{2 \pi}\right)^{2} \operatorname{tr}\left(R^{2}\right) \wedge\left(\operatorname{ch}_{0}(\mathcal{F}) C_{4}+\operatorname{ch}_{1}(\mathcal{F}) \wedge C_{2}\right)+\ldots \\
=-\frac{2 \pi}{l_{s}^{4}} \int_{\mathbb{R}^{3,1}} \frac{1}{96}\left(\frac{l_{s}^{2}}{2 \pi}\right)^{2} \operatorname{tr}\left(R^{2}\right) \wedge \\
\wedge\left[N_{\mathrm{D} 7} C_{I} m^{I}+\frac{1}{l_{s}^{4}} N_{\mathrm{D} 7} \mathcal{C}^{I} \int_{\Gamma_{\mathrm{D} 7}}\left(\frac{l_{s}^{2}}{2 \pi} \bar{f}+\varphi^{*} B_{2}^{+}\right) \wedge \omega_{I}\right]+\ldots,
\end{aligned}
$$

while from the O7-plane action, we infer the terms

$$
\begin{aligned}
\mathcal{S}_{\mathrm{O} 7}^{\mathrm{CS}} & =-\mu_{7} \kappa_{7} Q_{7} \int_{\mathrm{O} 7}\left(-\frac{1}{192}\left(\frac{l_{s}^{2}}{2 \pi}\right)^{2} \operatorname{tr}\left(R^{2}\right)\right) \wedge C_{4}+\ldots \\
& =-\frac{2 \pi}{l_{s}^{4}} \int_{\mathbb{R}^{3,1}} \frac{1}{24}\left(\frac{l_{s}^{2}}{2 \pi}\right)^{2} \operatorname{tr}\left(R^{2}\right) C_{I} m^{I}+\ldots
\end{aligned}
$$

A summary of the couplings relevant for the generalised Green-Schwarz mechanism in the present context can be found in table 7.2 , where we employed again the notion of Chern characters. 


\begin{tabular}{||clcl||}
\hline \hline$f_{a}-\mathcal{D}_{I}$ & $:$ & $\frac{l_{s}^{2}}{2 \pi} N_{\mathrm{D} 7_{a}} m_{a}^{I}$, \\
$f_{a}-D^{I}$ & $:$ & $\frac{l_{s}^{2}}{2 \pi} \frac{1}{l_{s}^{4}} \int_{\Gamma_{\mathrm{D} 7_{a}}} \operatorname{ch}_{1}\left(\overline{\mathcal{F}}_{a}^{+}\right) \wedge \omega_{I}$, \\
$f_{a}^{2}-\mathcal{C}^{I}$ & $:$ & $\left(\frac{l_{s}^{2}}{2 \pi}\right)^{2} \frac{1}{2} \frac{1}{l_{s}^{4}} \int_{\Gamma_{\mathrm{D} 7_{a}}} \operatorname{ch}_{1}\left(\overline{\mathcal{F}}_{a}^{+}\right) \wedge \omega_{I}$, \\
$f_{a}^{2}-C_{I}$ & $:$ & $\left(\frac{l_{s}^{2}}{2 \pi}\right)^{2} \frac{N_{\mathrm{D} 7_{a}}}{2} m_{a}^{I}$, \\
$\mathrm{F}_{a}^{2}-\mathcal{C}^{I}$ & $:$ & $\left(\frac{l_{s}^{2}}{2 \pi}\right)^{2} \frac{1}{4 N_{\mathrm{D} 7_{a}}} \frac{1}{l_{s}^{4}} \int_{\Gamma_{\mathrm{D} 7_{a}}} \operatorname{ch}_{1}\left(\overline{\mathcal{F}}_{a}^{+}\right) \wedge \omega_{I}$, \\
$\mathrm{F}_{a}^{2}-C_{I}$ & $:$ & $\left(\frac{l_{s}^{2}}{2 \pi}\right)^{2} \frac{1}{4} m_{a}^{I}$, \\
$R^{2}-\mathcal{C}^{I}$ & $:$ & $\left(\frac{l_{s}^{2}}{2 \pi}\right)^{2} \frac{1}{96} \frac{1}{l_{s}^{4}} \sum_{a, a^{\prime}} \int_{\Gamma_{\mathrm{D} 7_{a}}} \operatorname{ch}_{1}\left(\overline{\mathcal{F}}_{a}^{+}\right) \wedge \omega_{I}$, \\
$R^{2}-C_{I}$ & $:$ & $\left(\frac{l_{s}^{2}}{2 \pi}\right)^{2} \frac{1}{96}\left(\sum_{a, a^{\prime}} N_{\mathrm{D} 7_{a}} m_{a}^{I}+4 \sum_{\mathrm{O} 7_{i}} m_{\mathrm{O} 7_{i}}^{I}\right)$. \\
\hline \hline
\end{tabular}

Table 7.2: Summary of couplings relevant for the generalised Green-Schwarz mechanism in the context of type IIB orientifolds with D3- and D7-branes. Note that in $\overline{\mathcal{F}}^{+}$only the diagonally embedded $U(1)$ flux $(7.39)$ is turned on.

\subsubsection{Green-Schwarz Diagrams}

In this subsection, we now explicitly compute the Green-Schwarz diagrams illustrated in figure 4.1(b) and verify that these indeed cancel the chiral anomalies determined in section 7.2 .1 .

\section{Cubic Non-Abelian Anomaly}

For the cubic non-abelian anomaly, we see that there are no couplings of the form $\mathrm{F}-\mathcal{D}_{I}$ or $\mathrm{F}-D^{I}$ contained in the Chern-Simons actions (7.4). We therefore cannot construct the corresponding Green-Schwarz diagrams and so

$$
\mathcal{A}_{S U\left(N_{\mathrm{D} 7}\right)^{3}}^{\mathrm{GS}}=0 .
$$

This is expected since the cubic non-abelian anomaly 7.30 vanishes due to the tadpole cancellation conditions and does not need to be cancelled. 


\section{Mixed Abelian-Non-Abelian Anomaly}

Next, we consider the mixed abelian-non-abelian anomaly. The schematic form of the diagrams to be evaluated is

$$
\begin{array}{ll}
f_{a}-\mathcal{D}_{I}-\mathcal{C}^{I}-\mathrm{F}_{b}^{2}, & f_{a}-D^{I}-C_{I}-\mathrm{F}_{b}^{2}, \\
f_{a}-\mathcal{D}_{I}-\mathcal{C}^{I}-\mathrm{F}_{b^{\prime}}^{2}, & f_{a}-D^{I}-C_{I}-\mathrm{F}_{b^{\prime}}^{2},
\end{array}
$$

and with the help of the couplings shown in table 7.2 , we compute

$$
\begin{aligned}
\mathcal{A}_{U(1)_{a}-S U\left(N_{\mathrm{D} 7_{b}}\right)^{2}}^{\mathrm{GS}}= & \left(\frac{l_{s}^{2}}{2 \pi}\right)^{3} N_{\mathrm{D} 7_{a}} m_{a}^{I}(-1) \frac{1}{4 N_{\mathrm{D} 7_{b}}} \frac{1}{l_{s}^{4}} \int_{\Gamma_{\mathrm{D} 7_{b}}} \operatorname{ch}_{1}\left(\overline{\mathcal{F}}_{b}^{+}\right) \wedge \omega_{I} \\
& +\left(\frac{l_{s}^{2}}{2 \pi}\right)^{3} \frac{1}{l_{s}^{4}} \int_{\Gamma_{\mathrm{D} 7_{a}}} \operatorname{ch}_{1}\left(\overline{\mathcal{F}}_{a}^{+}\right) \wedge \omega_{I}(+1) \frac{1}{4} m_{b}^{I} \\
& +\left(b \rightarrow b^{\prime}\right) \\
= & \frac{1}{2}\left(\frac{l_{s}^{2}}{2 \pi}\right)^{3} \frac{N_{\mathrm{D} 7_{a}}}{2}\left(I_{a b}-I_{a^{\prime} b}\right)
\end{aligned}
$$

where we have used 4.3 as well as $m_{a}^{I} \omega_{I}=\left[\Gamma_{\mathrm{D} 7_{a}}\right]$. We also utilised that

$$
I_{a b^{\prime}}=-I_{a^{\prime} b}
$$

which is verified by employing (7.14) and noting that $\overline{\mathcal{F}}^{+}$is odd under $\Omega(-1)^{F_{L}}$. Comparing finally the Green-Schwarz contribution (7.47) to the anomaly (7.31), we see that up to a numerical prefactor, (7.47) cancels the mixed abelian-non-abelian anomaly.

\section{Cubic Abelian Anomaly}

For the cubic abelian anomaly, we need to compute the following Green-Schwarz diagrams

$$
\begin{array}{ll}
f_{a}-\mathcal{D}_{I}-\mathcal{C}^{I}-f_{b}^{2}, & f_{a}-D^{I}-C_{I}-f_{b}^{2}, \\
f_{a}-\mathcal{D}_{I}-\mathcal{C}^{I}-f_{b^{\prime}}^{2}, & f_{a}-D^{I}-C_{I}-f_{b^{\prime}}^{2} .
\end{array}
$$

Performing the same steps as for the mixed abelian-non-abelian anomaly, we arrive at

$$
\mathcal{A}_{U(1)_{a}-U(1)_{b}^{2}}^{\mathrm{GS}}=\frac{1}{2}\left(\frac{l_{s}^{2}}{2 \pi}\right)^{3} N_{\mathrm{D} 7_{a}} N_{\mathrm{D} 7_{b}}\left(I_{a b}-I_{a^{\prime} b}\right),
$$

and by comparing with 7.32 , we see that the Green-Schwarz contribution cancels the cubic abelian anomaly up to the same prefactor as for the mixed abelian-nonabelian anomaly. 


\section{Mixed Abelian-Gravitational Anomaly}

Finally, the mixed abelian-gravitational anomaly is computed schematically as

$$
f_{a}-\mathcal{D}_{I}-\mathcal{C}^{I}-R^{2}, \quad f_{a}-D^{I}-C_{I}-R^{2} .
$$

Utilising the couplings shown in table 7.2 as well as the D5- and D7-brane tadpole cancellation conditions, we find

$$
\begin{aligned}
\mathcal{A}_{U(1)_{a}-G^{2}}^{\mathrm{GS}}= & -\left(\frac{l_{s}^{2}}{2 \pi}\right)^{3} \frac{1}{96}\left[N_{\mathrm{D} 7_{a}} \sum_{b, b^{\prime}} \frac{1}{l_{s}^{4}} \int_{\Gamma_{\mathrm{D} 7_{b}}} \operatorname{ch}_{1}\left(\overline{\mathcal{F}}_{b}^{+}\right) \wedge\left[\Gamma_{\mathrm{D} 7_{a}}\right]\right. \\
& \left.-\frac{1}{l_{s}^{4}} \int_{\Gamma_{\mathrm{D} 7_{a}}} \operatorname{ch}_{1}\left(\overline{\mathcal{F}}_{a}^{+}\right) \wedge\left(\sum_{\mathrm{D} 7_{b}} N_{\mathrm{D} 7_{b}}\left(\left[\Gamma_{\mathrm{D} 7_{a}}\right]+\left[\Gamma_{\mathrm{D} 7_{b}}^{\prime}\right]\right)+4 \sum_{\mathrm{O} 7_{i}}\left[\Gamma_{\mathrm{O} 7_{i}}\right]\right)\right] \\
= & -\left(\frac{l_{s}^{2}}{2 \pi}\right)^{3} \frac{N_{\mathrm{D} 7_{a}}}{8} \sum_{\mathrm{O}_{i}} I_{\mathrm{O}_{i} a} .
\end{aligned}
$$

By comparing with (7.33), we see that up to a numerical prefactor, the contribution from the Green-Schwarz diagrams (7.52) has the right form to cancel the mixed abelian-gravitational anomaly.

\subsubsection{Massive U(1)s and Fayet-Iliopoulos Terms}

To conclude this section, let us comment on massive $U(1)$ factors and FayetIliopoulos terms.

\section{Massive U(1)s}

Using the definition of Chern characters given in (A.1), from equation (7.41) we can determine the Stückelberg mass terms for the gauge bosons on the D7-branes to be of the following form

$$
\begin{array}{r}
\mathcal{S}_{\text {mass }}=-\frac{1}{l_{s}^{2}} \int_{\mathbb{R}^{3,1}} \sum_{a, a^{\prime}} f_{\mathrm{D} 7_{a}} \wedge\left(N_{\mathrm{D} 7_{a}} m_{a}^{I} \mathcal{D}_{I}+\frac{1}{l_{s}^{4}} D^{I} \wedge \int_{\Gamma_{\mathrm{D} 7_{a}}} \operatorname{ch}_{1}\left(\overline{\mathcal{F}}_{\mathrm{D} 7_{a}}^{+}\right) \wedge \omega_{I}\right) \\
=-\frac{1}{l_{s}^{2}} \int_{\mathbb{R}^{3,1}} \sum_{\mathrm{D} 7_{a}} f_{\mathrm{D} 7_{a}} \wedge\left(N_{\mathrm{D} 7_{a}}\left(m_{a}^{I}-m_{a^{\prime}}^{I}\right) \mathcal{D}_{I}\right. \\
\left.\quad+\frac{1}{l_{s}^{4}} D^{I} \wedge \int_{\Gamma_{\mathrm{D} 7_{a}}} \operatorname{ch}_{1}\left(\overline{\mathcal{F}}_{\mathrm{D} 7_{a}}^{+}\right) \wedge\left(\omega_{I}+\sigma^{*} \omega_{I}\right)\right)
\end{array}
$$


where in going from the first to the second line we employed that the gauge field is odd under $\Omega(-1)^{F_{L}}$ together with equation (7.14). We then define the following two mass matrices for the gauge fields on the D7-branes

$$
M_{i a}=\frac{1}{l_{s}^{4}} \int_{\Gamma_{\mathrm{D} 7_{a}}} \operatorname{ch}_{1}\left(\overline{\mathcal{F}}_{a}^{+}\right) \wedge\left(\omega+\sigma^{*} \omega\right)_{i}, \quad M_{a}^{\hat{\imath}}=N_{\mathrm{D} 7_{a}}\left(m_{a}-m_{a^{\prime}}\right)^{\hat{\imath}}
$$

with $i=1, \ldots, h_{+}^{1,1}$ and $\hat{\imath}=1, \ldots, h_{-}^{1,1}$. Therefore, the massless (linear combinations of) $U(1)$ gauge fields on the D7-branes are those which are in the kernel of the combined matrix

$$
M_{I a}=\left[\begin{array}{c}
M_{i a} \\
\delta_{\hat{\imath} \hat{\jmath}} M_{a}^{\hat{\jmath}}
\end{array}\right] .
$$

Along the same lines as for the D7-branes, for the gauge fields on the D3-branes we find that due to the orientifold images, there are no mass terms

$$
\mathcal{S}_{\text {mass }}=-\frac{1}{l_{s}^{2}} \int_{\mathbb{R}^{3,1}} \sum_{b, b^{\prime}} f_{\mathrm{D} 3_{b}} \wedge \mathcal{D}_{0} N_{\mathrm{D} 3_{b}}=0 \text {. }
$$

\section{Fayet-Iliopoulos Terms}

Next, with the help of 7.54 , we can determine the form of the Fayet-Iliopoulos terms for the D7-branes. Observing that the mass matrices (7.54) correspond to the holomorphic Killing vectors of the gauged isometry associated to $T_{i}$ as well as to $G^{\hat{\imath}}$ and using the derivatives (3.23) of the Kähler potential, we can compute the Fayet-Iliopoulos terms in the following way

$$
\begin{aligned}
\xi_{a} & \sim M_{i a} \frac{\partial \mathcal{K}}{\partial T_{i}}-i M_{a}^{\hat{\imath}} \frac{\partial \mathcal{K}}{\partial G^{\hat{\imath}}} \\
& \sim-\frac{1}{2 l_{2}^{4}} \frac{e^{-\frac{\phi}{2}}}{\hat{\mathcal{V}}+\frac{\hat{\xi}}{2}}\left(2 \int_{\Gamma_{\mathrm{D} 7_{a}}} \operatorname{ch}_{1}\left(\overline{\mathcal{F}}_{a}^{+}\right) \wedge J+\int_{\left.\Gamma_{\mathrm{D} 7_{a}-\Gamma_{\mathrm{D} 7_{a}}^{\prime}} N_{\mathrm{D} 7_{a}} B_{2}^{-} \wedge J\right)}\right. \\
& \sim-\frac{1}{l_{2}^{4}} \frac{e^{-\frac{\phi}{2}}}{\hat{\mathcal{V}}+\frac{\hat{\xi}}{2}} \int_{\Gamma_{\mathrm{D} 7_{a}}} \operatorname{ch}_{1}\left(\overline{\mathcal{F}}_{a}\right) \wedge J
\end{aligned}
$$

where a sum over repeated indices $i$ and $\hat{\imath}$ is understood. Note that we employed the definition (A.1) as well as (7.14) together with (2.3), and we have not been careful with numerical prefactors.

Furthermore, in view of the D-term potential (3.7), in the case of vanishing VEVs for the matter fields we see that the Fayet-Iliopoulos term (7.57) in general leads 
to a non-zero D-term potential and thus supersymmetry is broken. In order for the D7-brane under consideration to preserve supersymmetry, the FI term (7.57) has to vanish which reproduces the supersymmetry condition already stated in equation 4.6

$$
\left.\left(J \wedge \operatorname{ch}_{1}(\overline{\mathcal{F}})\right)\right|_{\Gamma_{\mathrm{D} 7}}=0
$$

\subsection{D9- and D5-Branes for the O3-/O7-System}

In the preceding sections of this chapter, we have studied the tadpole cancellation conditions and the generalised Green-Schwarz mechanism for type IIB orientifolds with D3- and D7-branes. However, it is possible to introduce also D9- and D5branes which modify the tadpole cancellation conditions and therefore also the discussion for the chiral anomalies.

The reason for usually not considering D9- and D5-branes is that the orientifold projection maps them to anti-D9- and anti-D5-branes which are supersymmetric only at particular points in moduli space. Nevertheless, we can study the tadpole cancellation conditions and the chiral anomalies for such D-brane setups which we will do in some detail in the following.

\subsubsection{Tadpole Cancellation Conditions}

In order to determine the tadpole cancellation conditions, let us recall equation (7.10) and be more concrete about how the orientifold projection acts on the manifold a D9- or D5-brane is wrapping. In particular, we find

$$
\Gamma_{\mathrm{D} 9}^{\prime}=-\Gamma_{\mathrm{D} 9}, \quad \Gamma_{\mathrm{D} 5}^{\prime}=-\sigma \Gamma_{\mathrm{D} 5},
$$

where $\Gamma_{\mathrm{D} 9}=\mathcal{X}$ is invariant under the holomorphic involution $\sigma$ and $\Gamma_{\mathrm{D} 5}$ is a twocycle in $\mathcal{X}$ wrapped by a D5-brane. Furthermore, note that we are also allowed to turn on gauge flux $\bar{F}_{\text {D9 }}$ and $\bar{F}_{\text {D5 }}$ on the D9- respectively D5-branes which is odd under $\Omega(-1)^{F_{L}}$.

\section{D9-Brane Tadpole Cancellation Condition}

In a very similar way as in section 7.1, we can now compute the D9-brane tadpole cancellation condition. The variation of the Chern-Simons action (7.4) with respect 
to $C_{10}$ reads

$$
\delta_{C_{10}} \mathcal{S}_{\mathrm{D} 9}^{\mathrm{CS}}=-\frac{2 \pi}{l_{s}^{10}} \kappa_{9} \int_{\mathbb{R}^{3,1} \times \mathcal{X}} \delta C_{10} \wedge\left[\Gamma_{\mathrm{D} 9}\right] \wedge \mathrm{ch}_{0}\left(\overline{\mathcal{F}}_{\mathrm{D} 9}\right)
$$

where $\left[\Gamma_{\mathrm{D} 9}\right]$ is the Poincaré dual of $\Gamma_{\mathrm{D} 9}$ in $\mathcal{X}$, which is a zero-form, and the sign $\kappa_{9}$ had been introduced in equation $(2.32)$. Denoting the total number of D9-branes with gauge flux $\bar{F}_{a}$ by $N_{\mathrm{D} 9_{a}}=\operatorname{ch}_{0}\left(\mathcal{F}_{\mathrm{D} 9_{a}}\right)$, we find for the equation of motion originating from $C_{10}$ that

$$
0=\kappa_{9} \sum_{\mathrm{D} 9_{a}} N_{\mathrm{D}_{a}}\left(\left[\Gamma_{\mathrm{D}_{a}}\right]+\left[\Gamma_{\mathrm{D} 9_{a}}^{\prime}\right]\right)
$$

\section{D7-Brane Tadpole Cancellation Condition}

For the D7-brane tadpole cancellation condition, we compute the variation of the D9-brane Chern-Simons action with respect to $C_{8}$ as

$$
\delta_{C_{8}} \mathcal{S}_{\mathrm{D} 9}^{\mathrm{CS}}=-\frac{2 \pi}{l_{s}^{10}} \kappa_{9} \int_{\mathbb{R}^{3,1} \times \mathcal{X}} \delta C_{8} \wedge\left[\Gamma_{\mathrm{D} 9}\right] \wedge \operatorname{ch}_{1}\left(\overline{\mathcal{F}}_{\mathrm{D} 9}\right)
$$

Taking into account the orientifold images and combining $(7.62)$ with the variations of the D7-brane, O7-plane and bulk action (7.8) respectively (7.2), we find the following tadpole cancellation condition

$$
\kappa_{7} \sum_{a, a^{\prime}} N_{\mathrm{D} 7_{a}}\left[\Gamma_{\mathrm{D} 7_{a}}\right]+\kappa_{9} \sum_{b, b^{\prime}}\left[\Gamma_{\mathrm{D}_{b}}\right] \wedge \operatorname{ch}_{1}\left(\overline{\mathcal{F}}_{\mathrm{D} 9_{b}}\right)=8 \kappa_{7} \sum_{\mathrm{O} 7_{i}}\left[\Gamma_{\mathrm{O} 7_{i}}\right]
$$

where the prime denotes the image under the orientifold projection $\Omega(-1)^{F_{L}} \sigma$. However, in its present form $(7.63)$ still depends on the continuous fields $B_{2}^{-}$which is not desirable. But, writing out the first Chern character as

$$
\operatorname{ch}_{1}\left(\overline{\mathcal{F}}_{\mathrm{D} 9}\right)=\operatorname{ch}_{1}\left(\overline{\mathcal{F}}_{\mathrm{D} 9}^{+}\right)+N_{\mathrm{D} 9} B_{2}^{-}
$$

and noting that $B_{2}^{-}$is even under $\Omega(-1)^{F_{L}} \sigma$ while $\left[\Gamma_{\mathrm{D} 9}\right]$ is odd, we see that the dependence of $(7.63)$ on $B_{2}^{-}$vanishes. We can thus simply replace $\overline{\mathcal{F}} \rightarrow \overline{\mathcal{F}}^{+}$in the D7-brane tadpole cancellation condition above. 


\section{D5-Brane Tadpole Cancellation Condition}

Let us continue with the equation of motion for $C_{6}$. The variation of the D9-brane Chern-Simons action is computed as

$$
\delta_{C_{6}} \mathcal{S}_{\mathrm{D} 9}^{\mathrm{CS}}=-\frac{2 \pi}{l_{s}^{10}} \kappa_{9} \int_{\mathbb{R}^{3,1} \times \mathcal{X}} \delta C_{6} \wedge\left[\Gamma_{\mathrm{D} 9}\right] \wedge\left(\operatorname{ch}_{2}\left(\overline{\mathcal{F}}_{\mathrm{D} 9}\right)+l_{s}^{4} N_{\mathrm{D} 9} \frac{c_{2}(\mathcal{X})}{24}\right),
$$

where we observed that the tangential bundle of a D9-brane is equal to the tangential bundle of $\mathbb{R}^{3,1} \times \mathcal{X}$. The contribution of a D5-brane to the equation of motion of $C_{6}$ is found to be

$$
\delta_{C_{6}} \mathcal{S}_{\mathrm{D} 5}^{\mathrm{CS}}=-\frac{2 \pi}{l_{s}^{10}} \kappa_{5} \int_{\mathbb{R}^{3,1} \times \mathcal{X}} \delta C_{6} \wedge\left[\Gamma_{\mathrm{D} 5}\right] \wedge \operatorname{ch}_{0}\left(\overline{\mathcal{F}}_{\mathrm{D} 5}\right)
$$

where $\left[\Gamma_{\mathrm{D} 5}\right]$ denotes the Poincaré dual of $\Gamma_{\mathrm{D} 5}$ in $\mathcal{X}$. Taking into account the orientifold images and combining (7.65) as well as (7.66) with the variations computed in $(7.7)$ and $(7.2)$, we arrive at

$$
\begin{aligned}
& 0=\int_{\mathcal{X}} \omega_{I} \wedge\left[\quad \kappa_{7} \sum_{a, a^{\prime}}\left[\Gamma_{\mathrm{D} 7_{a}}\right] \wedge \operatorname{ch}_{1}\left(\varphi_{*} \overline{\mathcal{F}}_{\mathrm{D} 7_{a}}\right)\right. \\
& +\kappa_{9} \sum_{b, b^{\prime}}\left[\Gamma_{\mathrm{D}_{b}}\right] \wedge\left(\operatorname{ch}_{2}\left(\overline{\mathcal{F}}_{\mathrm{D}_{b}}\right)+l_{s}^{4} N_{\mathrm{D} 9_{b}} \frac{c_{2}(\mathcal{X})}{24}\right) \\
& \left.+\kappa_{5} \sum_{c, c^{\prime}}\left[\Gamma_{\mathrm{D} 5_{c}}\right] N_{\mathrm{D} 5_{c}}\right]
\end{aligned}
$$

where $\left\{\omega_{I}\right\}$ is again a basis of $(1,1)$-forms on $\mathcal{X}$. Since 7.67$)$ still depends on $B_{2}^{-}$, let us employ $(7.16)$ to separate out the $B_{2}^{-}$part from the first Chern character and use the definition A.1 to write the second Chern character as

$$
\operatorname{ch}_{2}\left(\overline{\mathcal{F}}_{\mathrm{D} 9}\right)=\operatorname{ch}_{2}\left(\overline{\mathcal{F}}_{\text {D9 }}^{+}\right)+\operatorname{ch}_{1}\left(\overline{\mathcal{F}}_{\text {D } 9}^{+}\right) \wedge B_{2}^{-}+\frac{N_{\mathrm{D} 9}}{2}\left(B_{2}^{-}\right)^{2} .
$$

Utilising then the D7-brane tadpole condition (7.63), we see that the dependence of 7.67$)$ on $B_{2}^{-}$vanishes, and so we can simply replace $\overline{\mathcal{F}} \rightarrow \overline{\mathcal{F}}^{+}$in (7.67).

\section{D3-Brane Tadpole Cancellation Condition}

To finish our discussion of the tadpole cancellation conditions, let us turn to the equation of motion for $C_{4}$. The variation of the D9-brane Chern-Simons action is 
calculated as

$$
\delta_{C_{4}} \mathcal{S}_{\mathrm{D} 9}^{\mathrm{CS}}=-\frac{2 \pi}{l_{s}^{10}} \kappa_{9} \int_{\mathbb{R}^{3,1} \times \mathcal{X}} \delta C_{4} \wedge\left[\Gamma_{\mathrm{D} 9}\right] \wedge\left(\operatorname{ch}_{3}\left(\overline{\mathcal{F}}_{\mathrm{D} 9}\right)+l_{s}^{4} \frac{c_{2}(\mathcal{X})}{24} \wedge \operatorname{ch}_{1}\left(\overline{\mathcal{F}}_{\mathrm{D} 9}\right)\right)
$$

while for the D5-brane action we find

$$
\delta_{C_{4}} \mathcal{S}_{\mathrm{D} 5}^{\mathrm{CS}}=-\frac{2 \pi}{l_{s}^{10}} \kappa_{5} \int_{\mathbb{R}^{3,1} \times \mathcal{X}} \delta C_{4} \wedge\left[\Gamma_{\mathrm{D} 5}\right] \wedge \operatorname{ch}_{1}\left(\varphi_{*} \overline{\mathcal{F}}_{\mathrm{D} 5}\right)
$$

Taking into account the orientifold images and combining the two expressions above with (7.6) as well as (7.2), we arrive at

$$
\begin{aligned}
4 N_{\text {flux }}= & \frac{\kappa_{7}}{l_{s}^{6}} \sum_{a, a^{\prime}} \int_{\mathcal{X}}\left[\Gamma_{\mathrm{D} 7_{a}}\right] \wedge\left(\operatorname{ch}_{2}\left(\varphi_{*} \overline{\mathcal{F}}_{\mathrm{D} 7_{a}}\right)+l_{s}^{4} N_{\mathrm{D} 7_{a}} \frac{c_{2}\left(\Gamma_{\mathrm{D} 7_{a}}\right)}{24}\right) \\
& +\frac{\kappa_{7}}{l_{s}^{6}} \sum_{\mathrm{O} 7_{i}} \int_{\mathcal{X}}\left[\Gamma_{\mathrm{O}_{i}}\right] \wedge\left(l_{s}^{4} \frac{c_{2}\left(\Gamma_{\mathrm{O} 7_{i}}\right)}{6}\right) \\
& +\frac{\kappa_{9}}{l_{s}^{6}} \sum_{b, b^{\prime}} \int_{\mathcal{X}}\left[\Gamma_{\mathrm{D}_{b}}\right] \wedge\left(\operatorname{ch}_{3}\left(\overline{\mathcal{F}}_{\mathrm{D}_{b}}\right)+l_{s}^{4} \operatorname{ch}_{1}\left(\overline{\mathcal{F}}_{\mathrm{D}_{b}}\right) \wedge \frac{c_{2}(\mathcal{X})}{24}\right) \\
& +\frac{\kappa_{5}}{l_{s}^{6}} \sum_{c, c^{\prime}} \int_{\mathcal{X}}\left[\Gamma_{\mathrm{D}_{c}}\right] \wedge \operatorname{ch}_{1}\left(\varphi_{*} \overline{\mathcal{F}}_{\mathrm{D}_{c}}\right) \\
& +\frac{\kappa_{3}}{l_{s}^{6}} \sum_{d, d^{\prime}} \int_{\mathcal{X}}\left[\Gamma_{\mathrm{D}_{d}}\right] N_{\mathrm{D} 3_{d}} \\
& +\frac{\kappa_{3}}{l_{s}^{6}} \sum_{\mathrm{O}_{3}} \int_{\mathcal{X}}\left[\Gamma_{\mathrm{O}_{j}}\right]\left(-\frac{1}{2}\right)
\end{aligned}
$$

where $\left[\Gamma_{\mathrm{D} 3}\right]=\mathcal{X}$ denotes the Poincaré dual of $\Gamma_{\mathrm{D} 3}$ in $\mathcal{X}$. Note that we have organised the appearing terms for later convenience. Similarly as in the previous cases, the dependence of this tadpole cancellation condition on the continuous fields $B_{2}^{-}$should vanish. And indeed, using the definition A.1), we can write the third Chern character as

$$
\operatorname{ch}_{3}\left(\overline{\mathcal{F}}_{\mathrm{D} 9}\right)=\operatorname{ch}_{3}\left(\overline{\mathcal{F}}_{\mathrm{D} 9}^{+}\right)+\operatorname{ch}_{2}\left(\overline{\mathcal{F}}_{\mathrm{D} 9}^{+}\right) \wedge B_{2}^{-}+\frac{1}{2} \operatorname{ch}_{1}\left(\overline{\mathcal{F}}_{\mathrm{D} 9}^{+}\right) \wedge\left(B_{2}^{-}\right)^{2}+\frac{N_{\mathrm{D} 9}}{3 !}\left(B_{2}^{-}\right)^{3}
$$


while for the first and second Chern character we use (7.64) respectively (7.68). The terms in (7.71) involving $B_{2}^{-}$can then be summarised as

$$
\begin{aligned}
& \frac{1}{l_{s}^{6}} \sum_{a, a^{\prime}} \int_{\mathcal{X}} B_{2}^{-} \wedge\left[\Gamma_{\mathrm{D} 7_{a}}\right] \wedge \operatorname{ch}_{1}\left(\varphi_{*} \overline{\mathcal{F}}_{\mathrm{D} 7_{a}}^{+}\right) \\
+ & \frac{\kappa_{9}}{l_{s}^{6}} \sum_{b, b^{\prime}} \int_{\mathcal{X}} B_{2}^{-} \wedge\left[\Gamma_{\mathrm{D} 9_{b}}\right] \wedge\left(\operatorname{ch}_{2}\left(\overline{\mathcal{F}}_{\mathrm{D} 9_{b}}^{+}\right)+l_{s}^{4} N_{\mathrm{D} 9_{b}} \frac{c_{2}(\mathcal{X})}{24}\right) \\
+ & \frac{\kappa_{5}}{l_{s}^{6}} \sum_{c, c^{\prime}} \int_{\mathcal{X}} B_{2}^{-} \wedge\left[\Gamma_{\mathrm{D} 5_{c}}\right] N_{\mathrm{D} 5_{c}},
\end{aligned}
$$

which cancel due to the D5-brane tadpole cancellation condition (7.67). In a very similar way as in section 7.1, we see that the terms in (7.71) proportional to $\left(B_{2}^{-}\right)^{2}$ have to vanish due to the D7-brane tadpole cancellation condition (7.63). Finally, using (7.72), we observe that the terms proportional to $\left(B_{2}^{-}\right)^{3}$ vanish, due to the tadpole cancellation condition (7.61). In $(7.71)$, we can therefore replace $\overline{\mathcal{F}} \rightarrow \overline{\mathcal{F}}^{+}$.

\subsubsection{Chiral Spectrum}

After having explicitly determined the tadpole cancellation conditions for a combined system of D9-, D7-, D5- and D3-branes, we will now formulate them in a more compact way. This will allow us to infer the rules for determining the chiral spectrum from the vanishing of the cubic non-abelian anomaly more easily.

\section{Summary of Tadpole Cancellation Conditions}

In order to express the tadpole cancellation conditions of the last subsection in a unified way, following for instance [109] (see also $[77,78,107]$ ), we define the charges ${ }^{2}$

$$
\begin{aligned}
\mathcal{Q}\left(\Gamma_{\mathrm{D} p}, \overline{\mathcal{F}}_{\mathrm{D} p}^{+}\right) & =\kappa_{p}\left[\Gamma_{\mathrm{D} p}\right] \wedge \operatorname{ch}\left(\varphi_{*} \overline{\mathcal{F}}_{\mathrm{D} p}^{+}\right) \wedge \sqrt{\frac{\hat{\mathcal{A}}\left(\mathcal{R}_{T_{\mathrm{D} p}}\right)}{\hat{\mathcal{A}}\left(\mathcal{R}_{N_{\mathrm{D} p}}\right)}}, \\
\mathcal{Q}\left(\Gamma_{\mathrm{O} p}\right) & =\kappa_{p} Q_{p}\left[\Gamma_{\mathrm{D} p}\right] \wedge \sqrt{\frac{\mathcal{L}\left(\mathcal{R}_{T_{\mathrm{D} p}} / 4\right)}{\mathcal{L}\left(\mathcal{R}_{N_{\mathrm{D} p}} / 4\right)}}
\end{aligned}
$$

\footnotetext{
${ }^{2}$ Note that we actually have to formulate these expressions in terms of sheaves. A naive way to compensate for this inaccuracy is to evaluate the Chern characters on the submanifold they are defined on whenever possible.
} 
The quantities involved here had been introduced around equation (7.4), but let us note once more that $[\Gamma]$ denotes the Poincaré dual of $\Gamma$ in $\mathcal{X}$, the $\mathrm{R}$-R charge of the O-planes was $Q_{p}=-2^{p-4}$ and that the signs $\kappa_{p}= \pm 1$ had been introduced in $(2.32)$.

By comparing the charges (7.74) with the explicit tadpole cancellation conditions (7.61), 7.63 and (7.67), we observe that the $\mathrm{D} p$-brane tadpoles for $p=9,7,5$ can be expressed in the following way

$$
0=\sum_{\mathrm{D} q, \mathrm{D} q^{\prime}} \mathcal{Q}\left(\Gamma_{\mathrm{D} q}, \overline{\mathcal{F}}_{\mathrm{D} q}^{+}\right)+\left.\sum_{\mathrm{O} q} \mathcal{Q}\left(\Gamma_{\mathrm{O} q}\right)\right|_{(9-p)-\text { form }} .
$$

In 7.75 , the restrictions selects to the zero-, two- and four-form part, and the sums in this and the following formulas run over all $\mathrm{D} p$-branes as well as over all $\mathrm{O} p$-planes. Concretely, this means

$$
\sum_{\mathrm{D} q, \mathrm{D} q^{\prime}}=\sum_{\mathrm{D} 9_{a}}+\sum_{\mathrm{D} 9_{a^{\prime}}}+\sum_{\mathrm{D} 7_{b}}+\ldots+\sum_{\mathrm{D} 3_{d^{\prime}}}, \quad \quad \sum_{\mathrm{O} q}=\sum_{\mathrm{O} 7_{i}}+\sum_{\mathrm{O} 3_{j}} .
$$

By comparing the charges (7.74) with the explicit form of the D3-brane tadpole (7.71), we see that, using (7.3), this condition can be expressed as

$$
-\bar{H}_{3} \wedge \bar{F}_{3}=\sum_{\mathrm{D} q, \mathrm{D} q^{\prime}} \mathcal{Q}\left(\Gamma_{\mathrm{D} q}, \overline{\mathcal{F}}_{\mathrm{D} q}^{+}\right)+\left.\sum_{\mathrm{O} q} \mathcal{Q}\left(\Gamma_{\mathrm{O} q}\right)\right|_{6-\text { form }} .
$$

\section{Rules for Determining the Chiral Spectrum}

Let us now state the rules for computing the chiral spectrum in the present context. These are inferred from the requirement that the cubic non-abelian anomaly should vanish using solely the tadpole cancellation condition. For that purpose, following for instance [109], we define

$$
\begin{aligned}
& I_{\mathrm{D} p \mathrm{D} q}=\frac{1}{N_{\mathrm{D} p} N_{\mathrm{D} q}} \int_{\mathcal{X}} \mathcal{Q}\left(\Gamma_{\mathrm{D} p}, \overline{\mathcal{F}}_{\mathrm{D} p}^{+}\right) \wedge \mathcal{Q}\left(\Gamma_{\mathrm{D} q},-\overline{\mathcal{F}}_{\mathrm{D} q}^{+}\right) \\
& I_{\mathrm{O} p \mathrm{D} q}=\frac{1}{N_{\mathrm{D} q}} \int_{\mathcal{X}} \mathcal{Q}\left(\Gamma_{\mathrm{O} p}\right) \wedge \mathcal{Q}\left(\Gamma_{\mathrm{D} q},-\overline{\mathcal{F}}_{\mathrm{D} q}^{+}\right) .
\end{aligned}
$$

Note that here the prefactor is again due to the fact that we are counting representations instead of the chiral number of massless excitations. The multiplicities of the bi-fundamental and the symmetric as well as anti-symmetric representations in terms of these indices are given in table 7.3 . 


\begin{tabular}{c||c} 
Representation & Multiplicity \\
\hline \hline$\left(\bar{N}_{\mathrm{D} p}, N_{\mathrm{D} q}\right)$ & $I_{\mathrm{D} p \mathrm{D} q}$ \\
$\left(N_{\mathrm{D} p}, N_{\mathrm{D} q}\right)$ & $I_{\mathrm{D} p^{\prime} \mathrm{D} q}$ \\
$S_{\mathrm{D} p}$ & $\frac{1}{2}\left(I_{\mathrm{D} p^{\prime} \mathrm{D} p}+\frac{1}{4} \sum_{\mathrm{O} q} I_{\mathrm{O} q \mathrm{D} p}\right)$ \\
$A_{\mathrm{D} p}$ & $\frac{1}{2}\left(I_{\mathrm{D} p^{\prime} \mathrm{D} p}-\frac{1}{4} \sum_{\mathrm{O} q} I_{\mathrm{O} q \mathrm{D} p}\right)$
\end{tabular}

Table 7.3: Rules for determining the chiral spectrum for a combined system of D9-, D7-, D5- and D3-branes in the context of type IIB orientifolds with O3- and O7-planes. The sums run over all O-planes as in equation (7.76).

\subsubsection{Chiral Anomalies}

The expressions in table 7.3 had been adjusted to the fact that the generalised Green-Schwarz mechanism does not provide any terms to cancel the cubic nonabelian anomaly. This anomaly therefore has to vanish due to the tadpole cancellation conditions which we verify now. In particular, using (7.28) and table 7.1, we compute

$$
\begin{aligned}
\mathcal{A}_{S U\left(N_{\mathrm{D} p}\right)^{3}}=\sum_{\mathrm{D} q \neq \mathrm{D} p} N_{\mathrm{D} q}\left(I_{\mathrm{D} q \mathrm{D} p}+I_{\mathrm{D} q^{\prime} \mathrm{D} p}\right) & +\left(N_{\mathrm{D} p}+4\right) \frac{1}{2}\left(I_{\mathrm{D} p^{\prime} \mathrm{D} p}+\frac{1}{4} \sum_{\mathrm{O} q} I_{\mathrm{O} q \mathrm{D} p}\right) \\
& +\left(N_{\mathrm{D} p}-4\right) \frac{1}{2}\left(I_{\mathrm{D} p^{\prime} \mathrm{D} p}-\frac{1}{4} \sum_{\mathrm{O} q} I_{\mathrm{O} q \mathrm{D} p}\right) \\
= & \sum_{\mathrm{D} q} N_{\mathrm{D} q}\left(I_{\mathrm{D} q \mathrm{D} p}+I_{\mathrm{D} q^{\prime} \mathrm{D} p}\right)+\sum_{\mathrm{O} q} I_{\mathrm{O} q \mathrm{D} p} \\
= & \frac{1}{N_{\mathrm{D} p}} \int_{\mathcal{X}}\left(\sum_{\mathrm{D} q, \mathrm{D} q^{\prime}} \mathcal{Q}\left(\Gamma_{\mathrm{D} q}, \overline{\mathcal{F}}_{\mathrm{D} q}^{+}\right)+\sum_{\mathrm{O} q} \mathcal{Q}\left(\Gamma_{\mathrm{O} q}\right)\right) \wedge \mathcal{Q}\left(\Gamma_{\mathrm{D} p},-\overline{\mathcal{F}}_{\mathrm{D} p}^{+}\right) .
\end{aligned}
$$

Employing then the tadpole cancellation conditions (7.75) and (7.77) together with the explicit form of the charges (7.74), we see that the anomaly 7.79 can be simplified to

$$
\begin{aligned}
& \mathcal{A}_{S U\left(N_{\mathrm{D} 3}\right)^{3}}=\mathcal{A}_{S U\left(N_{\mathrm{D} 5}\right)^{3}}=\mathcal{A}_{S U\left(N_{\mathrm{D} 7}\right)^{3}}=0 \\
& \mathcal{A}_{S U\left(N_{\mathrm{D} 9}\right)^{3}}=-\frac{\kappa_{9}}{N_{\mathrm{D} 9}} \int_{\Gamma_{\mathrm{D} 9}} H_{3} \wedge F_{3} \stackrel{\text { Freed-Witten }}{=} 0 .
\end{aligned}
$$


For D9-branes, the cubic non-abelian anomaly vanishes due to the Freed-Witten anomaly cancellation condition [50] shown in equation (4.10) which says that $H_{3}$ restricted to a D-brane has to be zero.

Along the same lines as in section 7.2.1, we can determine the mixed abelian-nonabelian, the cubic abelian and the mixed abelian-gravitational anomalies to be of the following form

$$
\begin{array}{ll}
\mathcal{A}_{U(1)_{\mathrm{D} p}-S U\left(N_{\mathrm{D} q}\right)^{2}} & =\frac{1}{2} \delta_{\mathrm{D} p, \mathrm{D} q} \mathcal{A}_{S U\left(N_{\mathrm{D} p}\right)^{3}}-\frac{1}{2} N_{\mathrm{D} q}\left(I_{\mathrm{D} p \mathrm{D} q}-I_{\mathrm{D} p^{\prime} \mathrm{D} q}\right), \\
\mathcal{A}_{U(1)_{\mathrm{D} p}-U(1)_{\mathrm{D} q}^{2}}=\frac{N_{\mathrm{D} p}}{3} \delta_{\mathrm{D} p, \mathrm{D} q} \mathcal{A}_{S U\left(N_{\mathrm{D} p}\right)^{3}}-N_{\mathrm{D} p} N_{\mathrm{D} q}\left(I_{\mathrm{D} p \mathrm{D} q}-I_{\mathrm{D} p^{\prime} \mathrm{D} q}\right), \\
\mathcal{A}_{U(1)_{\mathrm{D} p}-G^{2}}= & N_{\mathrm{D} p} \mathcal{A}_{S U\left(N_{\mathrm{D} p}\right)^{3}}-\frac{3}{4} N_{\mathrm{D} p} \sum_{\mathrm{O} q} I_{\mathrm{O} q \mathrm{D} p} .
\end{array}
$$

We are not going to show that the dimensional reduction of the Chern-Simons actions (7.4) provides the required Green-Schwarz couplings to cancel these anomalies. This can be done in a very similar way as in section 7.2 .

\subsubsection{Massive U(1)s and Fayet-Iliopoulos Terms}

We finish this section with a discussion of massive $U(1)$ s and the Fayet-Iliopoulos terms. For the case of D7- and D3-branes, this has been done in section 7.2.4 so here we will focus on the D5- and D9-branes. Furthermore, we will consider only diagonally embedded abelian fluxes on the D5- and D9-branes in order to simplify the discussion.

\section{Focus on D5-Branes}

To determine the couplings of the $U(1)$ gauge bosons associated to the D5-branes to the R-R $p$-form potentials $C_{p}$, let us expand the two-cycle the D5-brane is wrapping as

$$
\Gamma_{\mathrm{D} 5}=m_{\mathrm{D} 5 I} \Sigma^{I}
$$

where $\left\{\Sigma^{I}\right\}$ denotes the basis of two-cycles introduced in table 2.1. Writing then out the Chern characters as in equation (7.40), we obtain

$$
\begin{aligned}
\mathcal{S}_{\text {mass }} & =-\frac{\kappa_{5}}{l_{s}^{2}} \int_{\mathbb{R}^{3,1}} \sum_{a, a^{\prime}} f_{\mathrm{D} 5_{a}} \wedge\left(N_{\mathrm{D} 5_{a}} D^{I} \wedge \frac{1}{l_{s}^{2}} \int_{\Gamma_{\mathrm{D} 5_{a}}} \omega_{I}+\mathcal{D}_{0} \wedge \frac{1}{l_{s}^{2}} \int_{\Gamma_{\mathrm{D} 5_{a}}} \operatorname{ch}_{1}\left(\overline{\mathcal{F}}_{\mathrm{D} 5_{a}}^{+}\right)\right) \\
& =-\frac{\kappa_{5}}{l_{s}^{2}} \int_{\mathbb{R}^{3,1}} \sum_{a} f_{\mathrm{D} 5_{a}} \wedge\left(N_{\mathrm{D}_{a}}\left(m_{\mathrm{D} 5_{a} I}+m_{\mathrm{D} 5_{a^{\prime}} I}\right) D^{I}\right)
\end{aligned}
$$


where the term involving $\operatorname{ch}_{1}\left(\overline{\mathcal{F}}^{+}\right)$vanishes due to its orientifold image. The mass matrix for the $U(1)$ gauge bosons on the D5-branes therefore reads

$$
M_{i \mathrm{D} 5_{a}}=N_{\mathrm{D} 5_{a}}\left(m_{\mathrm{D} 5_{a}}+m_{\mathrm{D} 5_{a^{\prime}}}\right)_{i} .
$$

Recalling the derivative of the Kähler potential with respect to $T_{i}$ given in equation (3.23) and noting that (7.84) corresponds to the holomorphic Killing vectors of the gauged isometry associated to $T_{i}$, we can determine the form of the Fayet-Iliopoulos term of a D5-brane as

$$
\xi_{\mathrm{D} 5_{a}} \sim M_{i \mathrm{D} 5_{a}} \frac{\partial \mathcal{K}}{\partial T_{i}}=-\frac{1}{l_{s}^{2}} \frac{e^{-\frac{\phi}{2}}}{\hat{\mathcal{V}}+\frac{\hat{\xi}}{2}} \int_{\Gamma_{\mathrm{D} 5_{a}}} \operatorname{ch}_{0}\left(\overline{\mathcal{F}}_{\mathrm{D} 5_{a}}\right) J
$$

Note that in the case of vanishing VEVs for matter fields on the D5-brane or in the absence thereof, the Fayet-Iliopoulos term 7.85 in general gives rise to a non-vanishing D-term and so supersymmetry is broken. Only in the special case when 7.85 is vanishing, that means the D5-brane wraps a cycle of zero volume $\operatorname{Vol}\left(\Gamma_{\mathrm{D} 5}\right)=\int_{\Gamma_{\mathrm{D} 5}} J=0$, supersymmetry will be preserved.

\section{Focus on D9-Branes}

In order to study the mass matrix and Fayet-Iliopoulos terms for the D9-branes, let us expand the R-R eight-form potential $C_{8}$ in the following way

$$
C_{8}=D^{0} \wedge d \operatorname{vol}_{\mathcal{X}}
$$

Writing out the fourth Chern character similarly as in $(7.40$, we can determine the mass terms for the $U(1)$ gauge bosons on the D9-branes as

$$
\begin{aligned}
\mathcal{S}_{\text {mass }}=-\frac{\kappa_{9}}{l_{s}^{2}} \int_{\mathbb{R}^{3,1}} \sum_{a, a^{\prime}} f_{\mathrm{D} 9_{a}} \wedge\left[D^{0} \wedge\left[\Gamma_{\mathrm{D}_{a}}\right] N_{\mathrm{D} 9_{a}}\right. \\
+\mathcal{D}_{I} \wedge \frac{1}{l_{s}^{6}} \int_{\Gamma_{\mathrm{D} 9_{a}}} \sigma^{I} \wedge \operatorname{ch}_{1}\left(\overline{\mathcal{F}}_{\mathrm{D} 9_{a}}^{+}\right) \\
+D^{I} \wedge \frac{1}{l_{s}^{6}} \int_{\Gamma_{\mathrm{D} 9_{a}}} \omega_{I} \wedge\left(\operatorname{ch}_{2}\left(\overline{\mathcal{F}}_{\mathrm{D} 9_{a}}^{+}\right)+l_{s}^{4} N_{\mathrm{D} 9_{a}} \frac{c_{2}(\mathcal{X})}{24}\right) \\
\left.+\mathcal{D}_{0} \wedge \frac{1}{l_{s}^{6}} \int_{\Gamma_{\mathrm{D} 9_{a}}}\left(\operatorname{ch}_{3}\left(\overline{\mathcal{F}}_{\mathrm{D}_{a}}^{+}\right)+l_{s}^{4} \operatorname{ch}_{1}\left(\overline{\mathcal{F}}_{\mathrm{D} 9_{a}}^{+}\right) \frac{c_{2}(\mathcal{X})}{24}\right)\right] .
\end{aligned}
$$


Taking into account the explicit expression for the orientifold images, we see that the couplings $f_{\mathrm{D} 9} \wedge \mathcal{D}_{0}$ in the last line of (7.87) vanish. From the remaining terms, we determine the following mass matrices

$$
\begin{array}{ll}
f_{\mathrm{D} 9_{a}}-D^{0}: & M_{0 \mathrm{D} 9_{a}}=2 N_{\mathrm{D}_{a}}, \\
f_{\mathrm{D} 9_{a}}-\mathcal{D}_{I}: & M_{\mathrm{D} 9_{a}}^{\hat{\imath}}=\frac{1}{l_{s}^{6}} \int_{\mathcal{X}} \operatorname{ch}_{1}\left(\overline{\mathcal{F}}_{\mathrm{D} 9_{a}}^{+}\right) \wedge\left(\sigma^{I}-\sigma^{*} \sigma^{I}\right), \\
f_{\mathrm{D} 9_{a}}-D^{I}: & M_{i \mathrm{D} 9_{a}}=\frac{1}{l_{s}^{6}} \int_{\mathcal{X}}\left(\operatorname{ch}_{2}\left(\overline{\mathcal{F}}_{\mathrm{D} 9_{a}}^{+}\right)+l_{s}^{4} N_{\mathrm{D}_{a}} \frac{c_{2}(\mathcal{X})}{24}\right) \wedge\left(\omega+\sigma^{*} \omega\right)_{i},
\end{array}
$$

where $\sigma^{*} \sigma^{I}$ denotes the image of the basis four-form $\sigma^{I}$ under the holomorphic involution $\sigma$. The Fayet-Iliopoulos terms for the D9-branes are then computed similarly as in the previous cases using the derivatives (3.23) of the Kähler potential. Concretely, by employing (7.20) we find

$$
\begin{aligned}
\xi_{\mathrm{D} 9_{a}} \sim M_{i \mathrm{D} 9_{a}} \frac{\partial \mathcal{K}}{\partial T_{i}}-i M_{\mathrm{D} 9_{a}}^{\hat{\imath}} \frac{\partial \mathcal{K}}{\partial G^{\hat{\imath}}}+M_{0 \mathrm{D} 9_{a}} \frac{\partial \mathcal{K}}{\partial S} \\
\sim-\frac{e^{-\frac{\phi}{2}}}{\hat{\mathcal{V}}+\frac{\hat{\xi}}{2}}\left[N_{\mathrm{D} 9_{a}} e^{\frac{3}{2} \phi}(\hat{\mathcal{V}}+2 \hat{\xi})\right. \\
\left.\quad+\frac{1}{l_{s}^{6}} \int_{\mathcal{X}}\left(\operatorname{ch}_{2}\left(\overline{\mathcal{F}}_{\mathrm{D} 9_{a}}\right)+l_{s}^{4} N_{\mathrm{D} 9_{a}} \frac{c_{2}(\mathcal{X})}{24}\right) \wedge J\right] .
\end{aligned}
$$

\section{An Observation Concerning the Fayet-Iliopoulos Terms}

Let us now take a closer look at $(7.89)$ and recall the definitions introduced in equations 3.13), 3.14 and 3.22 . In particular, using $\frac{\zeta(2)}{(2 \pi)^{2}}=\frac{1}{24}$ with $\zeta(n)$ the Riemann $\zeta$-function as well as $\alpha^{\prime}=l_{s}^{2} /(2 \pi)^{2}$, we can rewrite 7.89 in the following way

$$
\begin{aligned}
\xi_{\mathrm{D} 9_{a}} \sim-\frac{1}{l_{s}^{6}} \frac{e^{-\frac{\phi}{2}}}{\hat{\mathcal{V}}+\frac{\hat{\xi}}{2}} \int_{\mathcal{X}}\left(\operatorname{ch}_{0}\left(\overline{\mathcal{F}}_{\mathrm{D} 9_{a}}\right)+\operatorname{ch}_{2}\left(\overline{\mathcal{F}}_{\mathrm{D} 9_{a}}\right)\right) \wedge e^{-J} \wedge \\
\left(1+\sum_{n=2,3}\left(2 \pi \alpha^{\prime}\right)^{n} \zeta(n) c_{n}(\mathcal{X})\right) .
\end{aligned}
$$

Note that the $\alpha^{\prime}$-correction to the Kähler potential encoded in $\xi$, that is the $n=3$ term in the sum above, fits together nicely with the $n=2$ term corresponding to the gravitational contribution to the Chern-Simons action of the D-branes. 
Finally, recalling from equations (7.57) and 7.85) the form of the Fayet-Iliopoulos term for the D7- respectively D5-branes, we can summarise them in the following way

$$
\begin{aligned}
& \xi_{\mathrm{D} 3} \sim 0 \\
& \xi_{\mathrm{D} 5} \sim-\frac{1}{l_{s}^{2}} \frac{e^{-\frac{\phi}{2}}}{\hat{\mathcal{V}}+\frac{\hat{\xi}}{2}} \int_{\Gamma_{\mathrm{D} 5}} \operatorname{ch}_{0}\left(\overline{\mathcal{F}}_{\mathrm{D} 5}\right) \wedge e^{J}, \\
& \xi_{\mathrm{D} 7} \sim-\frac{1}{l_{s}^{4}} \frac{e^{-\frac{\phi}{2}}}{\hat{\mathcal{V}}+\frac{\hat{\xi}}{2}} \int_{\Gamma_{\mathrm{D} 7}} \operatorname{ch}_{1}\left(\overline{\mathcal{F}}_{\mathrm{D} 7}\right) \wedge e^{J}, \\
& \xi_{\mathrm{D} 9} \sim-\frac{1}{l_{s}^{6}} \frac{e^{-\frac{\phi}{2}}}{\hat{\mathcal{V}}+\frac{\hat{\xi}}{2}} \int_{\Gamma_{\mathrm{D} 9}}\left(\operatorname{ch}_{0}\left(\overline{\mathcal{F}}_{\mathrm{D} 9}\right)+\operatorname{ch}_{2}\left(\overline{\mathcal{F}}_{\mathrm{D} 9}\right)\right) \wedge e^{-J} \wedge\left(1+\sum_{n=2,3}\left(2 \pi \alpha^{\prime}\right)^{n} \zeta(n) c_{n}\left(\Gamma_{\mathrm{D} 9}\right)\right) .
\end{aligned}
$$

Note that given the structure of the D9-brane FI term, one would expect for instance also $c_{2}\left(\Gamma_{\mathrm{D} 7}\right)$ corrections to the D7-brane result. In order to derive these, one has to redo the analysis of $[66,67]$ by taking into account the $\hat{\mathcal{A}}$-terms in the D-brane Chern-Simons action. However, this program is beyond the scope of this thesis.

\subsection{Summary}

In the present chapter and in chapter 4, we have studied and presented some aspects of model building constraints relevant for type IIB String Theory compactifications on orientifolds of compact Calabi-Yau manifolds with O3- and O7-planes.

- In particular, in chapter 4 we have given an overview on model building constraints relevant for type II orientifold constructions.

- In section 7.1 we have determined in detail the tadpole cancellation conditions in the context of orientifolds with O3- and O7-planes. We have seen that in the presence of O7-planes we are forced to introduce D7-branes, possibly endowed with gauge flux, and a combination of D3-branes and background flux.

Furthermore, for an orientifold projection $\Omega(-1)^{F_{L}} \sigma$ leading to $h_{-}^{1,1} \neq 0$, that is there are two- and four-cycles anti-invariant under the holomorphic involution $\sigma$, in general the D5-brane tadpole cancellation conditions lead to non-trivial constraints. This has already been mentioned in [145], however, here we have worked out this condition in detail.

- In section 4.2, we have summarised the rules for computing the chiral spectrum for a configuration of D3- and D7-branes. More concretely, we have seen that 
chiral matter is only possible for D7-branes which are endowed with gauge flux.

In section 7.2, we have then determined the chiral anomalies for a general configuration of D7-branes on a smooth Calabi-Yau three-fold, and we have shown that these anomalies are cancelled via the generalised Green-Schwarz mechanism. Note that the D7- as well as the D5-brane tadpole conditions were crucial in order to obtain this cancellation.

- Utilising the computation of the Stückelberg mass terms for the gauge bosons on the D7-branes, we were also able to determine the form of the FayetIliopoulos terms for D7-branes. In this way, we verified the supersymmetry condition (4.6) for D7-branes.

- Finally, in section 7.3, we have generalised the analysis of the preceding sections of this chapter by including also D9- and D5-branes. More concretely, we have worked out the tadpole cancellation conditions for this case in detail, and by utilising the requirement that these ensure the vanishing of the cubic non-abelian anomaly, we were able to determine a general set of rules for computing the chiral spectrum of the combined system of D9-, D7-, D5and D3-branes. These rules have been summarised in table 7.3. We have also computed the Fayet-Iliopoulos terms for D9- and D5-branes in such a setup, and observed an interesting structure. 


\section{Chapter 8}

\section{Applications of the D-Brane Instantons Calculus}

In this chapter, we are concerned with the second main topic of this thesis, namely D-brane instantons. After having given an introduction to the D-brane instanton calculus in chapter 5, we are now going to use this formalism to derive the so-called Affleck-Dine-Seiberg (ADS) superpotential in the context of String Theory, and we are going to derive a constraint for moduli stabilisation in type IIB orientifolds in the presence of an MSSM sector.

\subsection{The ADS Superpotential}

We begin with Affleck-Dine-Seiberg (ADS) superpotential [150] in order to illustrate the instanton calculus presented in chapter 5.

\section{The ADS Superpotential in Field Theory}

Let us first discuss the ADS superpotential in the Field Theory context. (For a nice and short review on this subject, please see [151].) We consider supersymmetric QCD (SQCD) which is a supersymmetric extension of QCD with $N_{\mathrm{f}}$ flavours of chiral superfields $\Phi_{\mathrm{f}}$ and $N_{\mathrm{f}}$ flavours of chiral anti-superfields $\widetilde{\Phi}_{\mathrm{f}}$. These are coupled to a $S U\left(N_{\mathrm{c}}\right)$ super Yang-Mills theory such that the $\Phi_{\mathrm{f}}$ transform in the fundamental and the $\widetilde{\Phi}_{\mathrm{f}}$ transform in the anti-fundamental respresentation of the gauge group $S U\left(N_{\mathrm{c}}\right)$. Therefore, $\Phi_{\mathrm{f}}$ and $\widetilde{\Phi}_{\mathrm{f}}$ can be written as matrix valued fields $\Phi_{\text {cf }}$ and $\widetilde{\Phi}_{\mathrm{fc}}$ where the so-called colour index takes values c $=1, \ldots, N_{\mathrm{c}}$ while for the flavour index we have $\mathrm{f}=1, \ldots, N_{\mathrm{f}}$. 
Concerning the superpotential, we take $W$ to vanish at the classical level which then implies, as it can be shown, that the superpotential is zero to all orders in perturbation theory. Therefore, the only possibility to obtain a non-vanishing contribution to $W$ is via non-perturbative effects. By employing the symmetries of the theory, one finds that such a non-perturbative superpotential has to be of the form

$$
W \sim(\operatorname{det}[\widetilde{\Phi} \Phi])^{-\frac{1}{N_{\mathrm{c}}-N_{\mathrm{f}}}}
$$

In the case of $N_{\mathrm{f}}<N_{\mathrm{c}}-1$, this superpotential is generated by gaugino condensation, however, in the situation where

$$
N_{\mathrm{f}}=N_{\mathrm{c}}-1
$$

the superpotential (8.1) is generated by instantons. This has been shown by Affleck, Dine and Seiberg in the paper [150] and thus the corresponding superpotential is usually abbreviated by ADS. Introducing then $\Lambda$ as the scale of the effective theory, on dimensional grounds the Affleck-Dine-Seiberg superpotential takes the form

$$
W_{\mathrm{ADS}}=\frac{\Lambda^{2 N_{\mathrm{c}}+1}}{\operatorname{det}[\widetilde{\Phi} \Phi]}
$$

\section{Engineering SQCD in String Theory}

In order to derive the ADS superpotential in the context of String Theory, let us now describe how to (locally) engineer SQCD via D-branes. Since the original paper [51] on the derivation of the ADS superpotential was formulated in terms of type IIA String Theory, in this section we switch from type IIB to type IIA, however, our discussion similarly applies to the type IIB case.

In the type IIA situation, we are considering again an orientifold of a CalabiYau three-fold $\mathcal{X}$ but this time introduce space-time filling D6-branes wrapping three-cycles in the compactification manifold. Since most of the model building techniques introduced in chapter 4 can straightforwardly be applied to the type IIA case, we do not present a detailled introduction to model building with D6branes but refer to the reviews $[34,35]$. In any case, here we consider the following configuration.

- We take a stack of $N_{\mathrm{c}}$ colour D6-branes wrapping a three-cycle $\Gamma_{\mathrm{c}}$ in $\mathcal{X}$ which is not invariant under the orientifold projection. In order to just obtain an $\mathcal{N}=1$ super Yang-Mills theory on these branes, we assume that the threecycle is rigid and preserves supersymmetry [152], that means $\Gamma_{\mathrm{c}}$ has to be special Lagrangian. 
- Next, we consider a stack of $N_{\mathrm{f}}$ flavour D6-branes wrapping a special Lagrangian cycle $\Gamma_{\mathrm{f}}$ in $\mathcal{X}$, again not invariant under the orientifold projection. Since we are interested in SQCD, we do not want chiral but vector-like bifundamental matter between these two stacks of branes.

Therefore, we assume that the intersections of the cycle $\Gamma_{\mathrm{c}}$ and $\Gamma_{\mathrm{f}}$ are such that there are precisely two representations $\Phi_{\mathrm{cf}}$ and $\tilde{\Phi}_{\mathrm{fc}}$ transforming as bifundamentals (of opposite chirality) under $U\left(N_{\mathrm{c}}\right) \times U\left(N_{\mathrm{f}}\right)$.

- Furthermore, due to the orientifold projection, we have to introduce the orientifold images of the colour and flavour branes. However, in order to obtain an SQCD theory, we assume that there are no intersections between the branes and their orientifold images as well as between the branes and the orientifold plane. In this way, symmetric or anti-symmetric chiral matter is absent.

- So far, the resulting gauge theory has gauge group $U\left(N_{\mathrm{c}}\right) \times U\left(N_{\mathrm{f}}\right)$. In order to obtain SQCD, we will first decouple the flavour gauge group by taking the volume of the cycle $\Gamma_{\mathrm{f}}$ to infinity while keeping the volume of $\Gamma_{\mathrm{c}}$ finite, that is

$$
\operatorname{Vol}\left(\Gamma_{\mathrm{f}}\right) \rightarrow \infty, \quad \operatorname{Vol}\left(\Gamma_{\mathrm{f}}\right)=\text { finite }
$$

Since $g_{\mathrm{f}}^{-2} \sim \operatorname{Vol}\left(\Gamma_{\mathrm{f}}\right)$, in this limit the gauge coupling on the flavour branes $g_{\mathrm{f}}$ vanishes and the $U\left(N_{\mathrm{f}}\right)$ theory decouples.

Also, generically the subgroup $U(1) \subset U\left(N_{\mathrm{c}}\right)$ acquires a mass due to the Green-Schwarz mass terms. (For type IIB orientifolds with O3- and O7-planes, we have determined these mass terms in section 7.2.2.) Therefore, we are left with a $S U\left(N_{\mathrm{c}}\right)$ gauge theory with $2 \times N_{\mathrm{f}}$ flavours.

- After having specified the matter sector of our configuration, let us now turn to the instanton. In particular, we consider an E2-brane instanton wrapping a cycle $\Gamma_{\mathcal{E}}$ which is located directly on top of $\Gamma_{\mathrm{c}}$, i.e. the E2-brane instanton is on top of the colour brane stack. The resulting zero-mode structure of this gauge instanton as well as the matter content of the configuration is summarised in figure 8.1 .

- Let us finally make a technical remark. In order to obtain the Field Theory result (8.3) and to neglect higher order instanton corrections, we are also taking the limit $\alpha^{\prime} \rightarrow 0$. In this approximation, we can then for instance ignore the one-loop amplitudes in the instanton correlation function (5.8) which will simplify our calculations. 


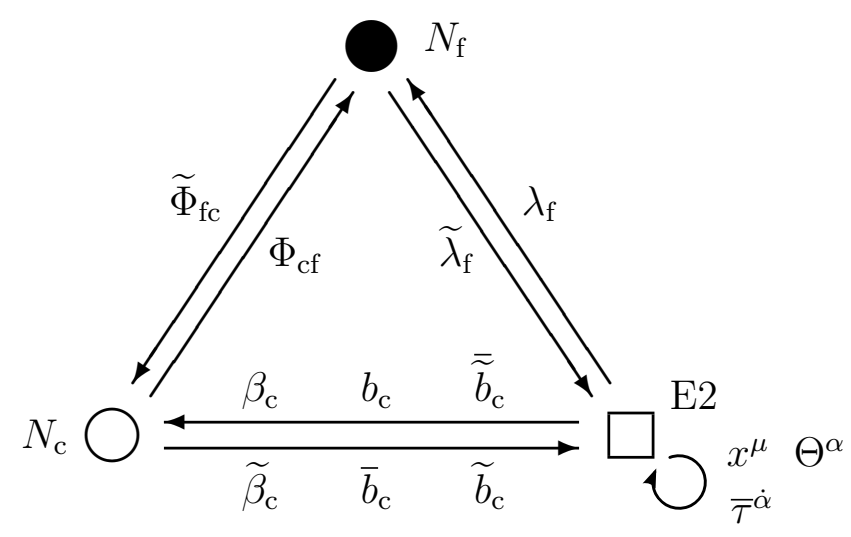

Figure 8.1: Local SQCD brane configuration with resulting zero-modes. The indices $\mathrm{c}$ and $\mathrm{f}$ take values $\mathrm{c}=1, \ldots, N_{\mathrm{c}}$ respectively $\mathrm{f}=1, \ldots, N_{\mathrm{f}}$. The $4 \times N_{\mathrm{c}}$ zero-modes $b$ are of bosonic nature while the $2 \times N_{\mathrm{c}}$ modes $\beta$ and the $2 \times N_{\mathrm{f}}$ modes $\lambda$ are fermionic. Finally, since we assume the instanton to not be invariant under the orientifold projection, also the $\bar{\tau}^{\dot{\alpha}}$ zero-modes are present.

\section{Disc Amplitudes and Supersymmetry Conditions}

After having explained the matter content and the instanton zero-mode structure of our configuration, let us now illustrate how the instanton zero-modes are absorbed in disc diagrams. Since the matter fields and (most of) the instanton zero-modes are described by boundary changing vertex operators, the disc diagrams to be evaluated correspond to closed loops in the quiver diagram 8.1. More concretely, the following contribution of disc diagrams will be relevant in the sequel

$$
\begin{aligned}
& \beta_{\mathrm{c}} \widetilde{\widetilde{\Phi}}_{\mathrm{cf}} \widetilde{\lambda}_{\mathrm{f}}+\lambda_{\mathrm{f}} \bar{\Phi}_{\mathrm{fc}} \widetilde{\beta}_{\mathrm{c}}, \\
& \frac{1}{2} b_{\mathrm{c}}\left(\Phi_{\mathrm{cf}} \bar{\Phi}_{\mathrm{fc}^{\prime}}+\overline{\widetilde{\Phi}}_{\mathrm{cf}} \widetilde{\Phi}_{\mathrm{fc}^{\prime}}\right) \bar{b}_{\mathrm{c}^{\prime}}+\frac{1}{2} \overline{\widetilde{b}}_{\mathrm{c}}\left(\Phi_{\mathrm{cf}} \bar{\Phi}_{\mathrm{fc}^{\prime}}+\overline{\widetilde{\Phi}}_{\mathrm{cf}} \widetilde{\Phi}_{\mathrm{fc}^{\prime}}\right) \widetilde{b}_{\mathrm{c}^{\prime}}, \\
& \bar{\tau}^{1}\left(\widetilde{\beta}_{\mathrm{c}} b_{\mathrm{c}}+\beta_{\mathrm{c}} \widetilde{b}_{\mathrm{c}}\right)+\bar{\tau}^{2}\left(\widetilde{\beta}_{\mathrm{c}} \overline{\widetilde{b}}_{\mathrm{c}}-\beta_{\mathrm{c}} \bar{b}_{\mathrm{c}}\right),
\end{aligned}
$$

where a sum over repeated indices is always understood. In addition to the disc amplitudes, there are D- and F-term constraints for the instanton to be supersymmetric which lead to the following expressions

$$
\sum_{\mathrm{c}=1}^{N_{\mathrm{c}}}\left[b_{\mathrm{c}} \widetilde{b}_{\mathrm{c}}+\bar{b}_{\mathrm{c}} \overline{\widetilde{b}}_{\mathrm{c}}\right]=0, \quad \sum_{\mathrm{c}=1}^{N_{\mathrm{c}}}\left[b_{\mathrm{c}} \widetilde{b}_{\mathrm{c}}-\bar{b}_{\mathrm{c}} \overline{\widetilde{b}}_{\mathrm{c}}\right]=0, \quad \sum_{\mathrm{c}=1}^{N_{\mathrm{c}}}\left[b_{\mathrm{c}} \bar{b}_{\mathrm{c}}-\widetilde{b}_{\mathrm{c}} \overline{\widetilde{b}}_{\mathrm{c}}\right]=0 .
$$

Note that after integrating over $\bar{\tau}$, equation (8.7) gives rise to the fermionic ADHM constraints for a gauge instanton, and equation (8.8) states the bosonic ADHM 
constraints [117]. We finally mention that the D-term constraint on the $N_{\mathrm{c}}$ colour branes leads to

$$
\bar{\Phi}=\widetilde{\Phi}, \quad \overline{\widetilde{\Phi}}=\Phi
$$

\section{Integration over Instanton Zero-Modes}

Given the expressions for the disc amplitudes from the previous paragraph, we can now compute correlation functions in the instanton background via the formalism presented in chapter 5. Out of these correlators, we construct the superpotential terms generated by the instanton. More concretely, in the Field Theory limit $\alpha^{\prime} \rightarrow 0$ mentioned before, in the present case we find

$$
\begin{aligned}
\mathcal{S}_{W} \sim \int d^{4} x d^{2} \Theta \prod_{\mathrm{c}=1}^{N_{\mathrm{c}}} & d^{4} b_{\mathrm{c}} d^{2} \beta_{\mathrm{c}} \prod_{\mathrm{f}=1}^{N_{\mathrm{f}}} d^{2} \lambda_{\mathrm{f}} \delta(\mathrm{ADHM}) \times \\
& \quad \times \exp \left(\mathcal{S}_{\mathrm{E} 2}\right) \exp (\beta \widetilde{\Phi} \widetilde{\lambda}+\lambda \Phi \widetilde{\beta}+b \Phi \widetilde{\Phi} \bar{b}+\overline{\widetilde{b}} \Phi \widetilde{\Phi} \widetilde{b})
\end{aligned}
$$

where the constraint (8.9) has already been employed and matrix products are understood. Furthermore, the integration over the $\bar{\tau}$ zero-modes has been performed leading, together with (8.8), to the ADHM constraints implemented by the $\delta$-function.

Now, performing the integration over the fermionic instanton zero-modes $\beta$ and $\lambda$ gives the condition

$$
N_{\mathrm{f}}=N_{\mathrm{c}}-1
$$

in order for (8.10) to be non-vanishing. In this way, we recover the well-known constraint of [150] mentioned in (8.2). The explicit computation for the fermionic zero-mode integration in (8.10) can be found in the original work [51] and leads to

$$
I_{\text {ferm. }}(\Phi, \widetilde{\Phi}, b, \widetilde{b})=\sum_{p, q=1}^{N_{\mathrm{c}}}(-1)^{p+q}\left(b_{p} \bar{b}_{q}+\overline{\widetilde{b}}_{p} \widetilde{b}_{q}\right) \operatorname{det}\left[\left.\Phi \widetilde{\Phi}\right|_{q, p}\right]
$$

where the symbol $\left.A\right|_{q, p}$ denotes the matrix obtained from $A$ by deleting the $q^{\prime}$ th row and $p$ 'th column. The integration over the bosonic instanton zero-modes $b$ schematically gives a factor of $(\operatorname{det}[\Phi \widetilde{\Phi}])^{-2}$ due to the Gaussian form of the integrand. Concretely, one finds

$$
I_{\text {bos. }}(\Phi, \widetilde{\Phi})=\frac{1}{\operatorname{det}[\widetilde{\Phi} \Phi]}
$$


Taking then into account the exponential of the instanton action, one arrives at

$$
\mathcal{S}_{W}=\frac{2 \pi^{2}}{l_{s}^{3}} \int d^{4} x d^{2} \Theta \frac{1}{\operatorname{det}[\widetilde{\Phi} \Phi]} \exp \left(-\frac{8 \pi^{2}}{g_{\mathrm{c}}^{2}\left(M_{\mathrm{s}}\right)}\right)
$$

where $g_{\mathrm{c}}\left(M_{s}\right)$ denotes the gauge coupling of the colour branes at the string scale $M_{s}$. Transforming then to canonically normalised fields via $\Phi \rightarrow\left(2 \pi \alpha^{\prime}\right)^{1 / 2} \Phi$ respectively $\widetilde{\Phi} \rightarrow\left(2 \pi \alpha^{\prime}\right)^{1 / 2} \widetilde{\Phi}$, introducing the dynamically generated scale $\Lambda$ as

$$
\left(\frac{\Lambda}{\mu}\right)^{3 N_{\mathrm{c}}-N_{\mathrm{f}}}=\exp \left(-\frac{8 \pi^{2}}{g_{\mathrm{c}}^{2}\left(M_{\mathrm{s}}\right)}\right)
$$

(where $\mu$ denotes the renormalisation group scale) and absorbing numerical prefactors into $\Lambda$, we can extract from (8.14) the ADS superpotential stated in equation 8.3

$$
W=\frac{\Lambda^{2 N_{\mathrm{c}}+1}}{\operatorname{det}[\widetilde{\Phi} \Phi]}
$$

\section{Summary}

We conclude this section with a brief summary.

- The final result (8.16) of our calculation in this section shows that indeed the ADS superpotential from Field Theory can be reproduced in String Theory. However, especially in view of absorbing instanton zero-modes, we have seen that we had to make a number of assumptions. In case these are not satisfied, a more detailled analysis is surely necessary. This implies in particular that one cannot simply add the ADS superpotential to String Theory configurations such as moduli stabilisation scenarios.

- Since for our configuration the ADS superpotential 8.16) is expected from Field Theory considerations, the computation outlined in this section is a non-trivial check of the instanton calculus developed in [54] and presented in section 5.2 .

- Furthermore, in our calculation we have taken a Field Theory limit in which for instance all massive states in the one-loop amplitudes disappear. In fact, when considering $\alpha^{\prime}$-corrections, the computation becomes much more involved as then also matter field insertion for the loop amplitudes become relevant.

- Finally, we mention that a similar analysis can be performed for orthogonal and symplectic gauge groups reproducing again the known results from Field Theory [51]. 


\subsection{A Chiral Zero-Mode Constraint}

After having discussed the ADS superpotential in the context of String Theory, thereby verifying the D-brane instanton calculus presented in chapter 5, we now turn to a constraint important for moduli stabilisation. Note that here we focus again on type IIB orientifolds with O3- and O7-planes but our discussion equally applies to other type II configurations.

\section{Starting Point}

In section 5.2, we have outlined the D-brane instanton calculus for type II orientifolds. In particular, in this prescription one has to integrate over the instanton zero-modes which have to be properly absorbed in disc and one-loop diagrams in order to give non-vanishing contributions. In the present section, we will be concerned with the chiral charged zero-modes and assume that the remaining instanton zero-modes have been taken care of.

More concretely, chiral charged instanton zero-modes in the context of type IIB orientifolds with D3- and D7-branes can originate from E3-brane instantons which is related to the fact that chiral matter is possible between D7-branes. We assume that such E3-instantons wrap supersymmetric rigid four-cycles $\Gamma_{\mathcal{E}}$ in the compactification manifold $\mathcal{X}$ which are invariant under the orientifold projection $\Omega(-1)^{F_{L}} \sigma$, that is

$$
\Gamma_{\mathcal{E}}=m_{\mathcal{E}}^{i} \gamma_{i} \in H_{4+}(\mathcal{X}, \mathbb{Z}), \quad \overline{\mathcal{F}}_{\mathcal{E}}^{+} \in H_{\bar{\partial}-}^{1,1}\left(\Gamma_{\mathcal{E}}\right),
$$

where $m_{\mathcal{E}}^{i} \in \mathbb{Z}$ are the wrapping numbers of the cycle $\Gamma_{\mathcal{E}}$ in the basis $\left\{\gamma_{i}\right\} \in$ $H_{4+}(\mathcal{X}, \mathbb{Z})$ introduced in table 2.1. Note that in general we are allowed to turn on "gauge flux" on the instanton provided that $\overline{\mathcal{F}}_{\mathcal{E}}^{+}$is invariant under the full orientifold projection. Therefore, in the single instanton case we are considering,

- the unwanted universal zero-modes $\bar{\tau}^{\hat{\alpha}}$ are absent since the instanton wraps a cycle invariant under the orientifold projection (and we assume that the would-be gauge group is $O(1)$ ).

- The moduli instanton zero-modes are absent since we assume that the cycle wrapped by the instanton to be rigid.

- We also assume that all vector-like charged instanton zero-modes are absent, or can be properly absorbed such that their contribution to the correlation function (5.8) is non-vanishing. We are thus left only with the chiral charged instanton zero-modes. 


\section{Chiral Charged Instanton Zero-Modes}

We now turn to the chiral charged instanton zero-modes in more detail. If there are D7-branes present in the setup, such modes are counted by the index (5.7). In order to study this expression, we define the matrices

$$
\begin{aligned}
& \mathcal{M}_{a I}=\frac{1}{l_{s}^{6}} \int_{\mathcal{X}} \operatorname{ch}_{1}\left(\varphi_{*} \overline{\mathcal{F}}_{a}^{+}\right) \wedge\left[\Gamma_{\mathrm{D} 7_{a}}\right] \wedge \omega_{I}, \\
& \mathcal{M}_{\mathcal{E} I}=\frac{1}{l_{s}^{6}} \int_{\mathcal{X}} \operatorname{ch}_{1}\left(\varphi_{*} \overline{\mathcal{F}}_{\mathcal{E}}^{+}\right) \wedge\left[\Gamma_{\mathcal{E}}\right] \wedge \omega_{I},
\end{aligned}
$$

where $\left\{\omega_{I}\right\} \in H_{\bar{\partial}}^{1,1}(\mathcal{X})$ denotes the basis of $(1,1)$-forms introduced in table 2.1. Using this definition, with $m_{a}^{I}$ the wrapping numbers of a D7-brane and a summation over repeated indices understood, we can express the index (5.7) as

$$
Z_{a \mathcal{E}}=\mathcal{M}_{a i} m_{\mathcal{E}}^{i}-\mathcal{M}_{\mathcal{E} I} m_{a}^{I}
$$

Let us now collect some more information about this expression. In particular, let us assume we have intersecting D7-branes in our setup which give rise to chiral matter. Expressing the index (4.3) counting chiral matter between D7-branes in terms of the matrices 8.18 , we find

$$
I_{a b}=\frac{\mathcal{M}_{a I}}{N_{\mathrm{D} 7_{a}}} m_{b}^{I}-\frac{\mathcal{M}_{b I}}{N_{\mathrm{D} 7_{b}}} m_{a}^{I} \neq 0
$$

for some D7-branes $a$ and $b$. From 8.20 it then follows that the rank of the matrix $\mathcal{M}$ has to be non-vanishing, that is

$$
\operatorname{rk}\left(\mathcal{M}_{a I}\right) \neq 0
$$

\section{Taking into Account an MSSM Sector}

Let us furthermore assume that in our setup the MSSM (or some extension of it) is realised via intersecting D7-branes with gauge flux. For simplicity, we focus on the case $h_{-}^{1,1}=0$ while the more subtle situation with $h_{-}^{1,1} \neq 0$ is discussed in appendix E.

In view of moduli stabilisation of Kähler moduli discussed in chapter 6, let us consider a setup with $h_{+}^{1,1}$ E3-instantons of type $O(1)$ wrapping cycles $\Gamma_{\mathcal{E}_{\Lambda}}$ with $\Lambda=1, \ldots, h_{+}^{1,1}$ which are all linearly independent. For instance, usually one takes $\Gamma_{\mathcal{E}_{\Lambda}}=\gamma_{i} \delta_{i \Lambda}$ with $\left\{\gamma_{i}\right\}$ the basis for $H_{4+}(\mathcal{X}, \mathbb{Z})$ introduced in table 2.1 so that all 
Kähler moduli appear in the superpotential via non-perturbative effects. Next, because $h_{-}^{1,1}=0$, we see from (8.17) that we cannot turn on flux $\overline{\mathcal{F}}_{\mathcal{E}}^{+}$on the instantons which for the matrix $\overline{\mathcal{M}}_{\mathcal{E} I}$ defined in equation (8.18) implies that

$$
\mathcal{M}_{\mathcal{E} I}=0
$$

With these assumptions and observations, we are now ready to present our main line of arguments.

- One of the important features of the Standard Model respectively the MSSM is chirality. In the present case this means that, as mentioned in 8.20), $I_{a b} \neq 0$ for some D7-branes $a$ and $b$ implying equation 8.21$)$, that is $\operatorname{rk}\left(\mathcal{M}_{a I}\right)$ is non-vanishing.

- Therefore, from 8.19 together with 8.22 we see that $\operatorname{rk}\left(\mathcal{M}_{a I}\right)$ of the $h^{1,1}$ linearly independent E3-instantons will have chiral charged instanton zeromodes with the MSSM D7-branes.

- In applying the formalism from section 5.2 , we see that if these instanton zeromodes can be absorbed in disc or one-loop diagrams leading to a non-vanishing contribution to the superpotential, it has to be of the form

$$
W \sim \prod_{i} \Phi_{i}^{\mathrm{MSSM}} e^{\mathcal{S}_{\mathrm{E} p}}
$$

for a string instanton. The reason for the appearance of the MSSM fields is illustrated in figure 8.2. In particular, since the chiral instanton zero-modes correspond to boundary changing vertex operators, the disc has to involve bi-fundamental MSSM matter fields.

- Finally, in order to not break the gauge symmetries, the MSSM fields $\Phi_{i}^{\mathrm{MSSM}}$ have to acquire a vanishing $\mathrm{VEV}$

$$
\left\langle\Phi_{i}^{\mathrm{MSSM}}\right\rangle=0
$$

implying that $(8.23)$ has to vanish. Thus, at least $\operatorname{rk}\left(\mathcal{M}_{a I}\right)$ of the $h^{1,1}$ linearly independent E3-instantons will not appear in the non-perturbative superpotential.

- Therefore, in the context of Kähler moduli stabilisation for type IIB orientifolds with O3- and O7-planes, at least $\operatorname{rk}\left(\mathcal{M}_{a I}\right)$ (linear combinations of) Kähler moduli do not appear in the non-perturbative superpotential and will not be stabilised via non-perturbative effects.

Clearly, in view of phenomenology this is a severe problem since, as we mentioned in the introduction to this work, moduli fields are not in agreement with experiment. However, we will provide a solution to this issue in chapter 9. 


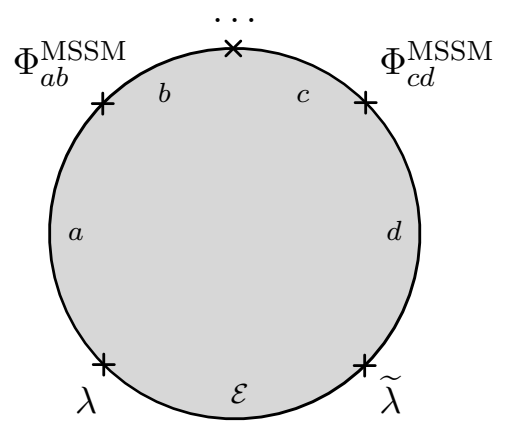

Figure 8.2: Illustration for the absorption of chiral MSSM charged instanton zeromodes. In particular, the MSSM branes are labelled by $a, b, \ldots, c, d$ and at least one of the $\Phi^{\mathrm{MSSM}}$ has to correspond to a boundary changing operator.

\section{A More Detailled Analysis}

In the last paragraph, we have presented our main line of arguments for the implication of an MSSM sector for instanton contributions to the superpotential. Let us now discuss this reasoning in some more detail.

- As mentioned before, the case with $h_{-}^{1,1} \neq 0$ is somewhat more subtle and is discussed in appendix E.

- Next, there are MSSM fields, namely the Higgs doublets, which do acquire non-vanishing vacuum expectation values. However, in this case a mass term of the generic order of the string scale will be generated via the D-term potential (3.5). Since the MSSM gauge symmetry breaking and mass generation should occur as usual at the low scale in the process of supersymmetry breaking, also the Higgs should acquire a vanishing VEV in our discussion.

- In the last paragraph, we have discussed the contribution of a string instanton to the superpotential. In the case of a gauge instanton, as we have seen in section 8.1, the generated terms in the superpotential are of the schematic form

$$
W \sim \frac{1}{\prod_{i} \Phi_{i}^{\mathrm{MSSM}}} e^{\mathcal{S}_{\mathrm{E} p}}
$$

As one can see, having a vanishing VEV for the MSSM fields is problematic. Therefore, such an instanton should not appear in the superpotential and thus the corresponding Kähler modulus will not be stabilised.

- As mentioned in section 8.1, very similar to the gauge instantons are gaugino condensates [153] which lead to a contribution of the same form as 8.25). However, gauge theories with gaugino condensation are not allowed to have 
chiral matter and therefore such a configuration cannot be part of the MSSM. In this way, we arrive at the same conclusion as for the instantons.

- Next, let us be more precise about the matter fields. There are in principle three types.

- There are chiral MSSM fields $\Phi^{\mathrm{MSSM}}$ transforming under the MSSM gauge group, or some extension thereof, such as $G=U(5) \times U(1)$, $G=U(4) \times U(2) \times U(2)$ or $G=U(3) \times U(2) \times U(1) \times U(1)$.

- But, there can be additional chiral fields which arise from the same set of intersecting D7-branes leading to so-called exotic matter fields $\Phi^{\text {exo }}$ transforming in non-trivial representation of the non-abelian part of the MSSM gauge group.

- In addition, in type II D-brane models one generically obtains extra $U(1)$ factors and so there can be fields $\Phi^{\text {abel }}$ which are not charged under the MSSM gauge group $S U(3) \times S U(2) \times U(1)_{Y}$ but carry non-trivial charges with respect to $U(1)$ s orthogonal to $U(1)_{Y}$.

Therefore, the instanton generated terms in the superpotential originating from string instantons in general take the following schematic form

$$
W \sim \prod_{i} \Phi_{i}^{\mathrm{MSSM}} \prod_{j} \Phi_{j}^{\mathrm{exo}} \prod_{k} \Phi_{k}^{\mathrm{abel}} e^{\mathcal{S}_{\mathrm{E} p}}
$$

Now, as we have explained, in order not to break the gauge symmetries at the string scale we require $\left\langle\Phi_{i}^{\mathrm{MSSM}}\right\rangle=\left\langle\Phi_{j}^{\text {exo }}\right\rangle=0$. Of course, in a complete model one would like to stabilise these matter fields in a dynamical way via soft supersymmetry breaking terms, perturbative and non-perturbative contributions to the superpotential as well as via D-terms at vanishing VEV. If such a mechanism is at work, since they appear in the same open string sector, it is likely that also the fields $\Phi_{k}^{\text {abel }}$ are fixed at zero vacuum expectation value. Therefore, with this assumption our general conclusion from the last paragraph is not changed.

- So far, we have focused on the MSSM sector of a D-brane configuration. In general, there exists a hidden sector possibly with further matter fields whose D- and F-terms however do not mix with the Standard Model sector. This sector can be important for instance for supersymmetry breaking, but it does not play a role in our discussion here. 


\subsection{Summary}

To conclude our discussion about instantons, let us briefly summarise the results obtained in chapter 5 and in the present chapter.

- In section 5.1, we have introduced our notation for the study of D-brane instantons and we have analysed the instanton action as well as its relation to closed string moduli fields. In particular, the action of a supersymmetric E3-brane instanton wrapped on a basis cycle $\gamma_{i} \in H_{4+}(\mathcal{X}, \mathbb{Z})$ contains the Kähler modulus $T_{i}$. Therefore, E3-brane instanton contributions to the nonperturbative superpotential depend on the Kähler moduli.

Furthermore, we have discussed instanton zero-modes and how these are counted.

- Next, in section 5.2 we have explained the instanton calculus developed in [54] and employed in [51]. In particular, we stated a formalism for the computation of correlation functions in the instanton background relevant for the superpotential, and we have discussed the absorption of instanton zero-modes as well as the schematic form of the resulting expressions.

- In section 8.1 of the present chapter, we have discussed an example for the instanton calculus being the ADS superpotential. In particular, we have given a setup locally realising SQCD, we have introduced an instanton, determined its zero-modes and applied the formalism from section 5.2. As expected, we obtained the ADS superpotential via a String Theory calculation [51], which provides a non-trivial check of the calculus given in [54].

- Finally, in section 8.2 we have analysed how instanton generated terms in type II String Theories are affected in the presence of an MSSM sector engineered by D-branes. In particular, we have argued on general grounds that

If the MSSM is realised by D-branes with gauge flux, then at most $\operatorname{def}\left(\mathcal{M}_{a I}\right)$ linearly independent E3-brane instantons give a nonvanishing contribution to the superpotential.

Here, we focused on the MSSM sector and the case $h_{-}^{1,1}=0$, and $\operatorname{def}\left(\mathcal{M}_{a I}\right)$ denotes the defect of the matrix $\mathcal{M}_{a I}$ defined in 8.18). This constraint has important implications for the usual mechanisms to stabilise closed string moduli discussed in chapter 6. In the next chapter, we are going to analyse this point in detail. 


\section{Chapter 9}

\section{The LARGE Volume Scenario with MSSM Sector}

We now come to one of the main results in this work which is the combination of the moduli stabilisation scenarios discussed in chapter 6 with an open string sector realising the MSSM. As we have seen in section 8.2, in the presence of the latter the contributions of D-brane instantons to the superpotential is restricted and thus, contrary to the common opinion, not all of the Kähler moduli can be stabilised in the usual way.

In this chapter, we are going to provide an approach of how to resolve this issue and we discuss an explicit example in the context of the LARGE Volume Scenario [52]. Note that although we will focus on the LVS, our reasoning equally applies to the KKLT scenario.

\subsection{General Analysis}

\section{Starting Point}

Before we actually begin, let us make clear our setup. In order to realise a LARGE Volume Scenario, we consider a manifold which

- admits a swiss-cheese structure of the form stated in equation 6.22 , that is, we assume the overall volume of the compactification manifold $\mathcal{X}$ to be expressible in terms of the four-cycle volumes $\tau_{I}$ in the following way

$$
\mathcal{V}=\eta_{\mathrm{b}}\left(\tau_{\mathrm{b}}\right)^{\frac{3}{2}}-\sum_{\tilde{\imath}=2}^{h^{1,1}} \eta_{\tilde{\imath}}\left(\tau_{\tilde{\imath}}\right)^{\frac{3}{2}}, \quad \quad \eta_{\mathrm{b}}, \eta_{\tilde{\imath}} \in \mathbb{R}^{+}
$$


- Furthermore, we assume the manifold $\mathcal{X}$ to have a strictly negative Euler characteristic in order to satisfy the second requirement for an LVS

$$
\chi(\mathcal{X})<0
$$

- and we assume that an orientifold projection can be chosen such that $h_{-}^{1,1}=0$ implying the absence of the $G^{\hat{\imath}}$-moduli defined in equation (2.16).

- Finally, we now take into account an open string sector, more concretely intersecting D7-branes with gauge flux, which realises the MSSM or some extension thereof. As we have seen in section 8.2, this implies that at least one of the Kähler moduli will not appear in the superpotential via non-perturbative effects. Therefore, in order to stabilise moduli via the LARGE Volume Scenario, we need at least one large Kähler modulus $T_{\mathrm{b}}$ and at least one small Kähler modulus supporting an instanton. We therefore have the constraint ${ }^{1}$

$$
h^{1,1} \geq 3
$$

With these assumptions, by the general reasoning presented in section 6.2, we expect that a LARGE Volume Scenario can be realised. This implies in particular, since $\hat{\mathcal{V}} \gg 1$, we can perform an expansion of the scalar potential in powers of $1 / \hat{\mathcal{V}}$.

Next, similar as in section 6.2, we assume that by a suitable choice of background fluxes $\bar{F}_{3}$ and $\bar{H}_{3}$ all complex structure moduli $U$ and the axio-dilaton $S$ can be fixed at leading order in $1 / \hat{\mathcal{V}}$ via the Gukov-Vafa-Witten superpotential (6.1) in a supersymmetric way. The corresponding F-term potential is displayed in (6.28), and minimising this expression leads to

$$
F_{U}=0, \quad F_{S}=0 .
$$

We finish this paragraph with one remark. As we have explained in section 4.5, when turning on background flux $\bar{H}_{3}$ and considering D-branes, one has to worry about the Freed-Witten anomaly. Therefore, we assume the D7-branes to wrap rigid four-cycles $\Gamma_{\mathrm{D} 7}$ in the compactification manifold $\mathcal{X}$ for which the pull-back of any three-form from the ambient space $\mathcal{X}$ onto the four-cycle $\Gamma_{\mathrm{D} 7}$ vanishes. This implies that the Freed-Witten anomaly is always cancelled, and in this way we also do not need to worry about open string moduli.

\footnotetext{
${ }^{1}$ For the KKLT scenario, the constraint reads $h_{+}^{1,1} \geq 2$ since in this case no big Kähler modulus is needed.
} 


\section{Taking Into Account the MSSM}

As we have just mentioned, in contrast to our discussion in chapter 6, we now assume that the MSSM or some extension thereof is realised via intersecting D7branes with gauge flux. In particular, we introduce D7-branes wrapping four-cycles

$$
\Gamma_{\mathrm{D} 7_{a}}=m_{a}^{I} \gamma_{I}
$$

in the internal manifold $\mathcal{X}$ where $a$ labels the MSSM branes and $\left\{\gamma_{I}\right\}$ is again the basis of four-cycles introduced in table 2.1. Note that for ease of our discussion, we only introduce MSSM D7-branes, but in general also hidden sector branes can be present.

Next, we recall the constraint developed in section 8.2. We introduce $h^{1,1}=h_{+}^{1,1}$ supersymmetric E3-brane instantons of type $O(1)$ wrapping linearly independent four-cycles

$$
\Gamma_{\mathcal{E}_{\Lambda}}=m_{\Lambda}^{I} \gamma_{I}
$$

where $\Lambda=1, \ldots, h^{1,1}$ labels the different instantons. Now, as we explained, there will be (at least) $\mathrm{rk}\left(\mathcal{M}_{a I}\right)$ of these instantons which have chiral charged zeromodes with the MSSM branes and thus their contribution to the superpotential, if non-vanishing, is of the form

$$
W \sim \prod_{i} \Phi_{i}^{\mathrm{MSSM}} e^{-2 \pi T_{\Lambda}}, \quad T_{\Lambda}=m_{\Lambda}^{I} T_{I} .
$$

Since the MSSM fields $\Phi_{i}^{\mathrm{MSSM}}$ should acquire a vanishing VEV, in general $\operatorname{rk}\left(\mathcal{M}_{a I}\right)$ (linear combinations of) Kähler moduli will not appear in the superpotential. With the assumptions from the beginning of this section, we then expect the following situation:

- due to the large volume $\mathcal{V} \gg 1$ and the swiss-cheese structure, the contribution of the big Kähler modulus to the non-perturbative superpotential can be neglected, but $\tau_{\mathrm{b}} \leftrightarrow \mathcal{V}^{2 / 3}$ will be stabilised perturbatively via the LVS mechanism.

- The def $\left(\mathcal{M}_{a I}\right)-1$ Kähler moduli which do appear in the superpotential via non-perturbative effects will be stabilised as in the usual LARGE Volume Scenario.

- The rk $\left(\mathcal{M}_{a I}\right)$ Kähler moduli which have charged instanton zero-modes with the MSSM branes will not appear in the non-perturbative superpotential and are thus not stabilised via non-perturbative effects. 


\section{The D-Term Potential}

As we have emphasised several times, moduli which are not stabilised are problematic for phenomenology since this situation is not in agreement with experiment. Therefore, also the $\operatorname{rk}\left(\mathcal{M}_{a I}\right)$ Kähler moduli mentioned in the last item of the list above have to be fixed. One way to do so is the following.

In the presence of additional gauge symmetries realised by D-branes, generically the D-term potential (3.5) is non-vanishing. More concretely, for diagonal gauge kinetic functions, canonically normalised matter fields and $U(1)$ gauge symmetry, the D-term potential reads (3.7)

$$
V_{D}=\sum_{a} \frac{1}{2 \operatorname{Re}\left(f_{a}\right)}\left(\sum_{m} Q_{a}^{m}\left|\Phi_{m}\right|^{2}-\xi_{a}\right)^{2} .
$$

Specialising then to D7-branes and utilising the definition of the matrix $\mathcal{M}_{a I}$ from equation (8.18), we can express the Fayet-Iliopoulos term $\xi_{a}$ shown in equation (7.57) as

$$
\xi_{a} \sim-\frac{1}{l_{2}^{4}} \frac{e^{-\frac{\phi}{2}}}{\hat{\mathcal{V}}+\frac{\hat{\xi}}{2}} \int_{\Gamma_{\mathrm{D} 7_{a}}} \operatorname{ch}_{1}\left(\overline{\mathcal{F}}_{a}\right) \wedge J=-\frac{1}{\hat{\mathcal{V}}+\frac{\hat{\xi}}{2}} \mathcal{M}_{a I} \hat{t}^{I}
$$

where a summation over repeated indices is again understood. Now, if the D7branes under consideration realise the MSSM, the fields $\Phi_{m}$ appearing in the Dterm potential (9.8) are MSSM matter fields $\Phi_{m}^{\mathrm{MSSM}}$ which have to acquire a vanishing vacuum expectation value in order to preserve the MSSM gauge symmetries.

Therefore, employing equation 3.33 for the gauge kinetic function of a D7-brane, the D-term potential above simplifies to

$$
V_{D} \simeq \sum_{a} \frac{1}{2 \hat{\tau}_{a}} \frac{1}{\hat{\mathcal{V}}^{2}}\left(\mathcal{M}_{a I} \hat{t}^{I}\right)^{2}
$$

where $\hat{\tau}_{a}=m_{a}^{I} \hat{\tau}_{I}$ denotes the volume of the four-cycle wrapped by the D7-brane $a$ (in Einstein frame). From this expression, we see that besides the F-term potential also the D-term potential depends on the Kähler moduli, however, only implicitly via $t^{I}=t^{I}\left(T_{J}\right)$.

\section{Discussion}

Let us now discuss the how in the presence of an MSSM sector, the D-term potential (9.10) may change the moduli stabilisation procedure in the LARGE Volume Scenario. 
- Recalling the D-term potential (9.10) where the matter fields have vanishing vacuum expectation value, we see that $V_{D}$ is minimised by $\hat{\mathcal{V}} \rightarrow \infty$ and $\hat{\tau}_{a} \rightarrow$ $\infty$ which corresponds to the decompactification limit we are not interested in. However, 9.10 is also minimised by

$$
0=\mathcal{M}_{a I} \hat{t}^{I}
$$

which fixes those $t^{I} \notin \operatorname{ker}\left(\mathcal{M}_{a I}\right)$ at $t^{I}=0$. Unfortunately, generically this implies that the $t^{I}$ are stabilised at the boundary of the Kähler cone meaning that some two- respectively four-cycle volumina of the compactification manifold $\mathcal{X}$ may vanish. In this situation, the supergravity approximation we are employing is no longer applicable and thus genuine String Theory effects such as world-sheet instantons have to be taken into account. The latter are then expected to fix the $t^{I} \notin \operatorname{ker}\left(\mathcal{M}_{a I}\right)$ at a small but finite value.

Furthermore, in the situation where some of the matter fields $\Phi_{m}$ in the Dterm potential acquire a non-vanishing VEV, for instance when the fields $\Phi^{\text {abel }}$ introduced on page 123 are present, one obtains a relation between these fields and the $t^{I}$ implying that the boundary of the Kähler cone can be avoided in the stabilisation procedure.

- We also observe that the D-term potential 9.10 is of order $1 / \hat{\mathcal{V}}^{2}$. Therefore, since (9.10) is positive definite, the combined scalar F- and D-term potential at order $\mathcal{O}\left(\hat{\mathcal{V}}^{-2}\right)$ is given by the sum of (6.28) and (9.10). This expression is minimised by vanishing F-terms for the complex structure moduli $U$ as well as the axio-dilaton $S$, and for a vanishing Fayet-Iliopoulos parameter.

- Note that the Kähler moduli $T_{I}$ appear in the D-term potential only implicitly via $t^{I}=t^{I}\left(T_{J}\right)$. A priori it is not clear whether exactly those $T_{I}$ "missing" in the F-term potential contribute to the D-term potential, but further information about the compactification manifold is necessary.

- To summarise, in the case of an MSSM sector realised by D-branes, only those (linear combinations of) small Kähler moduli

$$
T_{\Lambda}=m_{\Lambda}^{\tilde{\imath}} T_{\tilde{\imath}} \quad \text { with } \quad m_{\Lambda}^{\tilde{\imath}} \in \operatorname{ker}\left(\mathcal{M}_{a I}\right)
$$

(for fixed $\Lambda$ ) can appear in the non-perturbative superpotential and are stabilised via the LVS. However, since $\operatorname{rk}\left(\mathcal{M}_{a I}\right) \neq 0$, there is a non-trivial contribution to the D-term potential (9.10) and Kähler moduli $T_{I}$ appear implicitly in $V_{D}$. We thus find the following situation 


\begin{tabular}{|c|c|c|}
\hline Number & Moduli & Stabilisation \\
\hline 1 & Kähler modulus $\operatorname{Re}\left(T_{\mathrm{b}}\right)$ & stabilised perturbatively via LVS \\
\hline $\operatorname{def}\left(\mathcal{M}_{a I}\right)-1$ & small Kähler moduli & stabilised non-perturbatively via LVS \\
\hline $\operatorname{rk}\left(\mathcal{M}_{a I}\right)$ & small Kähler moduli & may be stabilised perturbatively via $V_{D}$ \\
\hline
\end{tabular}

Therefore, in principle we can stabilise all Kähler moduli via the combination of the F- and D-term potential. (For the stabilisation of $\operatorname{Im}\left(T_{\mathrm{b}}\right)$, please see the comment on page 78 .)

\subsection{An Example: The $\mathrm{P}_{[1,3,3,3,5]}[15]$ Calabi-Yau}

In this section, we are going to discuss a concrete example where the ideas outline in the previous section are realised. This example is the swiss-cheese manifold $\mathbb{P}_{[1,3,3,3,5]}[15]$ which has been studied in [52].

\subsubsection{Geometry and Topology}

Let us first describe the geometry and topology of the compactification manifold $\mathbb{P}_{[1,3,3,3,5]}[15]$. Further details, especially the toric resolution and the computation of co-homology groups, can be found in the original paper [52]. Here, we only summarise the information needed in the following.

\section{Hodge Numbers and Euler Characteristic}

As it turns out, the non-trivial Hodge numbers of the manifold $\mathbb{P}_{[1,3,3,3,5]}[15]$ are $\left(h^{1,1}, h^{2,1}\right)=(3,75)$ and therefore the Euler characteristic of this Calabi-Yau manifold is found as 2

$$
\chi(\mathcal{X})=2\left(h^{1,1}-h^{2,1}\right)=-144
$$

Since this number is negative, one of the requirements in 6.36 for a LARGE Volume Scenario is satisfied. Assuming furthermore that an orientifold projection can be chosen such that $h_{+}^{1,1}=h^{1,1}=3$, we see that there are three Kähler moduli present in our setup.

\footnotetext{
${ }^{2}$ The Euler number of a real $n$-dimensional manifold is computed in terms of the Betti numbers $b^{p}$ as $\chi=\sum_{p=0}^{n}(-1)^{p} b^{p}$. In case the manifold is complex, the Betti numbers $b^{p}$ are related to the Hodge numbers $h^{p, q}$ as $b^{p}=\sum_{r+s=p} h^{r, s}$.
} 


\section{Triple Intersection Numbers and Four-Cycle Volumes}

Next, let us denote a basis of divisors on $\mathcal{X}$, that is holomorphic four-cycles, as $\left\{\Gamma_{1}, \Gamma_{2}, \Gamma_{3}\right\}$. In this basis, as it turns out, the triple intersection numbers $k_{I J K}$ defined in (3.9) take the following form

$$
\begin{array}{lll}
k_{111}=9, & k_{112}=-15, & k_{113}=0, \\
k_{122}=25, & k_{123}=0, & k_{133}=0, \\
k_{222}=-40, & k_{223}=-5, & k_{233}=15, \\
k_{333}=-40 . & &
\end{array}
$$

With the help of these numbers, we can compute the four-cycle volumes $\tau_{I}$ defined in equation (3.12). In particular, expanding the Kähler form $J$ as

$$
J=\sum_{I=1}^{3} t^{I}\left[\Gamma_{I}\right]
$$

where $\left[\Gamma_{I}\right]$ denotes the Poincaré dual of $\Gamma_{I}$ in $\mathcal{X}$, we find for the $\tau_{I}$ that

$$
\begin{aligned}
\tau_{1} & =\frac{1}{2}\left(3 t^{1}-5 t^{2}\right)^{2}, \\
\tau_{2} & =\frac{5}{6}\left[\left(3 t^{3}-t^{2}\right)^{2}-\left(5 t^{2}-3 t^{1}\right)^{2}\right], \\
\tau_{3} & =-\frac{5}{2}\left(t^{2}-4 t^{3}\right)\left(t^{2}-2 t^{3}\right) .
\end{aligned}
$$

\section{Kähler Cone Constraint}

Now we are going to determine the Kähler cone which is defined by the condition that the volumes of all so-called effective curves $\mathcal{C}$ in $\mathcal{X}$ are positive. The first step is to compute the cone of all effective curves, which is called the Mori cone, and then deduce from this the Kähler cone by the condition $\int_{\mathcal{C}} J>0$. The resulting constraints describing the Kähler cone are

$$
t^{2}-2 t^{3}>0, \quad t^{1}-2 t^{2}+t^{3}>0, \quad-3 t^{1}+5 t^{2}>0 \text {. }
$$

These conditions also ensure hat the overall volume $\mathcal{V}$ is positive, that all volumes of effective divisors are positive and, by construction, that all volumes of effective holomorphic curves are positive. 


\section{Swiss-Cheese Structure}

Let us next determine the overall volume $\mathcal{V}$ of the compactification manifold $\mathcal{X}$. In the definition $(3.13), \mathcal{V}$ is expressed in terms of the expansion coefficients $t^{I}$ of the Kähler form (9.15). However, for our study of the LARGE Volume Scenario we need to express the overall volume in terms of the four-cycle volumes $\tau_{I}$. Since $\tau_{I} \sim\left(t^{I}\right)^{2}$, in general this is a non-trivial task.

In our particular example, employing (9.16) and (9.17), one finds that the overall volume of the compactification manifold can be written as

$$
\mathcal{V}=\sqrt{\frac{2}{45}}\left(\left(5 \tau_{1}+3 \tau_{2}+\tau_{3}\right)^{3 / 2}-\frac{1}{3}\left(5 \tau_{1}+3 \tau_{2}\right)^{3 / 2}-\frac{\sqrt{5}}{3}\left(\tau_{1}\right)^{3 / 2}\right)
$$

This expression shows that the manifold we are considering indeed admits a swisscheese structure since we can make $\tau_{3}$ large so that $\mathcal{V}$ becomes large while keeping the other four-cycles small. On the latter, the MSSM will be realised.

\section{Rigid Divisors}

Since we are going to consider D-brane instantons wrapping four-cycles in the internal manifold $\mathcal{X}$, it is useful to know the rigid divisors in $\mathbb{P}_{[1,3,3,3,5]}[15]$. In particular, wrapping E3-instantons on such rigid four-cycles, the moduli instanton zero-modes are absent allowing the instanton to contribute to the superpotential more easily.

Since determining whether a cycle is rigid amounts to the computation of the cohomology groups $H^{i}\left(\Gamma, \mathcal{O}_{\Gamma}\right)$ for $i=1,2$, we would have to introduce additional techniques which is beyond the scope of this thesis. We therefore simply state the rigid divisors in our setup and refer to the paper [52] for the detailled calculations. The rigid divisors of $\mathbb{P}_{[1,3,3,3,5]}[15]$ are

$$
\Gamma_{1}, \quad \Gamma_{1}+\Gamma_{2}, \quad 2 \Gamma_{1}+\Gamma_{2}, \quad 2 \Gamma_{1}+2 \Gamma_{2} .
$$

\section{Diagonal Basis}

Finally, in order to simplify our following discussion, let us introduce a diagonal basis in which the overall volume $\mathcal{V}$ as well as the triple intersection numbers take a particularly simple form. Guided by (9.18), we define

$$
\Gamma_{a}=5 \Gamma_{1}+3 \Gamma_{2}+\Gamma_{3}, \quad \Gamma_{b}=5 \Gamma_{1}+3 \Gamma_{2}, \quad \Gamma_{c}=\Gamma_{1},
$$


for which the triple intersection numbers $k_{I J K}$ diagonalise. More concretely, the only non-vanishing numbers are

$$
k_{a a a}=5, \quad k_{b b b}=45, \quad k_{c c c}=9 .
$$

The overall volume of the compactification manifold in terms of the divisor volumes $\tau_{a}, \tau_{b}$ and $\tau_{c}$ is then found as

$$
\mathcal{V}=\sqrt{\frac{2}{45}}\left(\tau_{a}^{3 / 2}-\frac{1}{3} \tau_{b}^{3 / 2}-\frac{\sqrt{5}}{3} \tau_{c}^{3 / 2}\right)
$$

Expanding also the Kähler form in this diagonal basis as

$$
J=t^{a}\left[\Gamma_{a}\right]-t^{b}\left[\Gamma_{b}\right]-t^{c}\left[\Gamma_{c}\right],
$$

with the relation between $\left\{t^{a}, t^{b}, t^{c}\right\}$ and $\left\{t^{1}, t^{2}, t^{3}\right\}$ inferred from 9.20 as

$$
t^{1}=5 t^{a}-5 t^{b}-t^{c}, \quad t^{2}=3 t^{a}-3 t^{b}, \quad t^{3}=t^{a},
$$

we find that the Kähler cone conditions (9.17) take the very simple form

$$
\frac{1}{3} t^{a}>t^{b}>t^{c}>0
$$

\subsubsection{Constructing the Open String Sector}

After having summarised the geometry and topology of our compactification manifold $\mathbb{P}_{[1,3,3,3,5]}[15]$, we are now going to address the question of how to construct the open string sector for our model.

\section{Ansatz for the MSSM Branes and the Instantons}

As we can see from equation (9.20), the large cycle in the diagonal basis is $\Gamma_{a}$ since it is the only one containing $\Gamma_{3}$. Therefore, in order to have realistic gauge couplings on the D7-branes as well as significant E3-brane instanton contributions to the superpotential, we make the following ansatz

$$
\Gamma_{\mathrm{D} 7}=n_{b} \Gamma_{b}+n_{c} \Gamma_{c}, \quad \Gamma_{\mathrm{E} 3}=m_{b} \Gamma_{b}+m_{c} \Gamma_{c},
$$

where now the wrapping numbers $n$ and $m$ need not be integer. They are related to the wrapping numbers $n_{i}$ in the $\left\{\Gamma_{1}, \Gamma_{2}, \Gamma_{3}\right\}$ basis as

$$
n_{b}=\frac{1}{3} n_{2}, \quad n_{c}=n_{1}-\frac{5}{3} n_{2} .
$$


Next, in order to keep our discussion simple, we are not attempting to actually construct the MSSM or some extension thereof but only consider a toy model with two stacks of D7-branes labelled by $A$ and $B$, where only on the first one a non-vanishing gauge flux $\overline{\mathcal{F}}_{A}$ is turned on.

\section{D-Term and Chiral Zero-Mode Constraint}

As we have explained in section 9.1, in order to minimise the combined scalar potential, the D-term potential $V_{D}$ has to vanish. Assuming that the vacuum expectation values of the MSSM fields originating from the branes $A$ and $B$ vanish, we see from (9.10) that the Fayet-Iliopoulos terms $\xi_{A}$ and $\xi_{B}$ have to be zero. Since we do not turn on flux on the D7-branes $B$ (and employing $h_{-}^{1,1}=0$ ), it follows that $\xi_{B}$ is trivially vanishing. However, for $\xi_{A}$ we find from (7.57) the D-term constraint

$$
0=\frac{1}{l_{s}^{6}} \int_{\mathcal{X}} \operatorname{ch}_{1}\left(\varphi_{*} \overline{\mathcal{F}}_{A}\right) \wedge\left[\Gamma_{A}\right] \wedge J
$$

Let us also consider the chiral zero-mode constraint developed in section 8.2 for an E3-instanton. In particular, using the explicit form of the index given in (8.19), the only non-trivial requirement originates from the D7-branes $A$ and reads

$$
0=\frac{1}{l_{s}^{6}} \int_{\mathcal{X}} \operatorname{ch}_{1}\left(\varphi_{*} \overline{\mathcal{F}}_{A}\right) \wedge\left[\Gamma_{A}\right] \wedge\left[\Gamma_{\mathrm{E} 3}\right]
$$

Now, using our ansatz 9.26 for the E3-brane instanton cycle in the diagonal basis, we find that the only suitable solution to the two equations above is

$$
J=t^{a}\left[\Gamma_{a}\right]-\alpha t\left[\Gamma_{\mathrm{E} 3}\right],
$$

where $t \in \mathbb{R}$ and $\alpha>0$ is some constant. From equation 9.23 together with (9.26), we see that this solution implies

$$
t^{b}=\alpha m_{b} t, \quad t^{c}=\alpha m_{c} t .
$$

Comparing with the Kähler cone constraint $t^{b}>t^{c}>0$ shown in equation 9.25 and going back to the basis $\left\{\Gamma_{1}, \Gamma_{2}, \Gamma_{3}\right\}$ via 9.27 , we deduce that only wrapping numbers satisfying

$$
2 m_{2}>m_{1}>\frac{5}{3} m_{2}
$$

are possible. Finally, from equation $(9.19)$ we then see that this condition is not met by any of the rigid divisors in our configuration. Therefore, in our example it is not possible to simultaneously satisfy the D-term constraint (9.28), the chiral zero-mode constraint 9.29 and the Kähler cone constraint 9.32). 


\section{D-Brane and Instanton Configuration}

However, relaxing the requirement of being strictly inside the Kähler cone, we see that wrapping the E3-instanton on the divisor $2 \Gamma_{1}+\Gamma_{2}$ leads to a solution at the boundary of the Kähler cone. Therefore, transforming into the diagonal basis, we observe that the E3-brane instanton suitable for our purpose is given by

$$
\Gamma_{\mathrm{E} 3}=\frac{1}{3}\left(\Gamma_{b}+\Gamma_{c}\right) .
$$

Of course, we cannot choose instantons at will but have to take all of them into account. But we can arrange our setup in such a way that only a rigid instanton along the cycle 9.33 will contribute to the non-perturbative superpotential.

We now turn to the D7-branes. In particular, we choose the stacks of branes to wrap the following rigid four-cycles

$$
\Gamma_{A}=\Gamma_{1}+\Gamma_{2}=\frac{1}{3}\left(\Gamma_{b}-2 \Gamma_{c}\right), \quad \Gamma_{B}=\Gamma_{1}=\Gamma_{c},
$$

with abelian gauge flux specified by

$$
\operatorname{ch}_{1}\left(\varphi_{*} \overline{\mathcal{F}}_{A}\right)=\frac{N_{A}}{3}\left(2 \Gamma_{b}+5 \Gamma_{c}\right), \quad \quad \operatorname{ch}_{1}\left(\varphi_{*} \overline{\mathcal{F}}_{B}\right)=0
$$

where $N_{A}$ denotes the number of D7-branes in the stack $A$. With this choice, there are no chiral charged instanton zero-modes between the D7-branes and the E3-instanton (9.33). Indeed, evaluating (9.29) for the branes $A$ we find

$$
\begin{aligned}
& \frac{1}{l_{s}^{6}} \int_{\mathcal{X}} \operatorname{ch}_{1}\left(\varphi_{*} \overline{\mathcal{F}}_{A}\right) \wedge\left[\Gamma_{A}\right] \wedge\left[\Gamma_{\mathrm{E} 3}\right] \\
= & \frac{1}{l_{s}^{6}} \int_{\mathcal{X}} \frac{N_{A}}{3}\left(2\left[\Gamma_{b}\right]+5\left[\Gamma_{c}\right]\right) \wedge \frac{1}{3}\left(\left[\Gamma_{b}\right]-2\left[\Gamma_{c}\right]\right) \wedge \frac{1}{3}\left(\left[\Gamma_{b}\right]+\left[\Gamma_{c}\right]\right) \\
= & \frac{1}{l_{s}^{6}} \frac{N_{A}}{27} \int_{\mathcal{X}}\left(2\left[\Gamma_{b}\right]^{3}-10\left[\Gamma_{c}\right]^{3}\right) \\
= & \frac{N_{A}}{27}(2 \cdot 45-10 \cdot 9)=0
\end{aligned}
$$

where we employed the explicit expression for the D-brane and instanton cycles as well as the triple intersection numbers (9.21) in the diagonal basis. Since we do not turn on gauge flux for the D7-branes $B$, we see that equation $(9.29)$ is trivially satisfied, and so there are no chiral charged instanton zero-modes.

However, in general there are also vector-like zero-modes present. One way to remove these would be to turn on discrete Wilson lines on the D7-brane respectively the E3-instanton. From now on, we simply assume that such non-chiral zero-modes can be made massive and that indeed the E3-instanton given by (9.33) contributes to the superpotential. 


\section{Other Rigid E3-Brane Instantons}

To conclude this subsection, let us now check that indeed the E3-brane instanton wrapping the cycle (9.33) is the only rigid instanton satisfying the chiral zero-mode constraint (9.29). In particular, following the same steps as in the computation (9.36), we find that the chiral zero-mode constraint reads

$$
0=10 N_{A}\left(m_{b}-m_{c}\right)=10 N_{A}\left(2 m_{2}-m_{1}\right)
$$

By comparing with (9.19), we see that this condition is indeed only solved by the instanton we are considering while the others always have chiral charged instanton zero-modes.

\subsubsection{Moduli Stabilisation}

After having motivated how to engineer the open string sector consisting of the matter D7-branes as well as an E3-brane instanton for our model, we now turn to the stabilisation of the Kähler moduli of the background manifold $\mathbb{P}_{[1,3,3,3,5]}[15]$.

\section{D-Term Potential}

As we have mentioned before, at order $1 / \hat{\mathcal{V}}^{2}$ the combined scalar potential is minimised by vanishing F-term and D-term potentials. Since we assume zero vacuum expectation values for the matter fields, the vanishing of $V_{D}$ corresponds to vanishing Fayet-Iliopoulos terms. Let us therefore evaluate equation (9.28) for our example specified by 9.34 and 9.35 . Employing the triple intersection numbers (9.21) in the diagonal basis, we compute

$$
\begin{aligned}
0 & =\frac{1}{l_{s}^{6}} \int_{\mathcal{X}} \operatorname{ch}_{1}\left(\varphi_{*} \overline{\mathcal{F}}_{A}\right) \wedge\left[\Gamma_{A}\right] \wedge J \\
& =\frac{1}{l_{s}^{6}} \int_{\mathcal{X}} \frac{N_{A}}{3}\left(2\left[\Gamma_{b}\right]+5\left[\Gamma_{c}\right]\right) \wedge \frac{1}{3}\left(\left[\Gamma_{b}\right]-2\left[\Gamma_{c}\right]\right) \wedge\left(t^{a}\left[\Gamma_{a}\right]-t^{b}\left[\Gamma_{b}\right]-t^{c}\left[\Gamma_{c}\right]\right) \\
& =\frac{1}{l_{s}^{6}} \frac{N_{A}}{9} \int_{\mathcal{X}}\left(-2 t^{b}\left[\Gamma_{b}\right]^{3}+10 t^{c}\left[\Gamma_{c}\right]^{3}\right) \\
& =-10 N_{A}\left(t^{b}-t^{c}\right)
\end{aligned}
$$

which reproduces the solution already given in equation 9.30 , namely

$$
t^{b}=t^{c}=: t
$$


Going then back to the basis $\left\{\Gamma_{1}, \Gamma_{2}, \Gamma_{3}\right\}$ by using the relations given in (9.24), we see from equations $(9.16)$ that $(9.39)$ implies

$$
\begin{array}{lll}
\tau_{1}=\frac{9}{2}\left(t^{c}\right)^{2} & \stackrel{9.39}{\longrightarrow} \frac{9}{2}(t)^{2}, \\
\tau_{2}=\frac{15}{2}\left(\left(t^{b}\right)^{2}-\left(t^{c}\right)^{2}\right) & \stackrel{9.39}{\longrightarrow} 0, \\
\tau_{3}=\frac{5}{2}\left(\left(t^{a}\right)^{2}-9\left(t^{b}\right)^{2}\right) & \stackrel{9.39}{\longrightarrow} & \frac{5}{2}\left(\left(t^{a}\right)^{2}-9(t)^{2}\right) .
\end{array}
$$

Therefore, the D-term constraint always fixes the moduli such that the four-cycle $\Gamma_{2}$ shrinks to zero size while the cycle $\Gamma_{1}$ as well as the large cycle $\Gamma_{3}$ stay finite. Please note that our MSSM branes do both involve $\Gamma_{1}$ and so their volume and hence gauge coupling is always non-zero and finite, respectively.

\section{F-Term Potential}

After having discussed the D-term constraint at order $1 / \hat{\mathcal{V}}^{2}$ in the large volume expansion, let us now turn to the F-term potential at order $1 / \hat{\mathcal{V}}^{3}$. In particular, assuming that the complex structure moduli $U$ as well as the axio-dilaton $S$ are stabilised via background flux by $F_{U}=F_{S}=0$, from equation (6.31) we recall the formula for scalar F-term potential an specialise to the single instanton case

$$
\begin{aligned}
V_{F}=\frac{g_{s}}{2} \varrho[\quad & -\frac{4}{\hat{\mathcal{V}}} \widehat{\operatorname{Vol}}\left(\Gamma_{\mathrm{E} 3} \cap \Gamma_{\mathrm{E} 3}\right) a_{\mathrm{E} 3}^{2}\left|\mathcal{A}_{\mathrm{E} 3}\right|^{2} e^{-2 a_{\mathrm{E} 3} \hat{\tau}_{\mathrm{E} 3}} \\
& +\frac{2}{\hat{\mathcal{V}}^{2}} \hat{\tau}_{\mathrm{E} 3} a_{\mathrm{E} 3}\left(\mathcal{A}_{\mathrm{E} 3} e^{-a_{\mathrm{E} 3} T_{\mathrm{E} 3}} \bar{W}_{0}+\text { c.c. }\right) \\
& \left.+\frac{3}{4} \frac{\hat{\xi}}{\hat{\mathcal{V}}^{3}}\left|W_{0}\right|^{2}\right]+\mathcal{O}\left(\hat{\mathcal{V}}^{-4}\right) .
\end{aligned}
$$

Next, we stabilise the axion $\operatorname{Im}\left(T_{\mathrm{E} 3}\right)$ similarly as explained in section 6.2 , and we compute using the results developed above

$$
\begin{aligned}
\operatorname{Vol}\left(\Gamma_{\mathrm{E} 3} \cap \Gamma_{\mathrm{E} 3}\right) & =\frac{1}{l_{s}^{6}} \int_{\mathcal{X}}\left[\Gamma_{\mathrm{E} 3}\right] \wedge\left[\Gamma_{\mathrm{E} 3}\right] \wedge J \\
& =-\frac{1}{9} \frac{1}{l_{s}^{6}} \int_{\mathcal{X}}\left(t^{b}\left[\Gamma_{b}\right]^{3}+t^{c}\left[\Gamma_{c}\right]^{3}\right) \\
& =-\frac{1}{9}\left(45 t^{b}+9 t^{c}\right) \\
& =-5 t^{b}-t^{c}=-\sqrt{\frac{2}{9}}\left(\sqrt{5 \tau_{b}}+\sqrt{\tau_{c}}\right)
\end{aligned}
$$


where we utilised that $t^{b}=\sqrt{2 / 9} \sqrt{\tau_{b} / 5}$ as well as that $t^{c}=\sqrt{2 / 9} \sqrt{\tau_{c}}$. Using then the D-term constraint from equation (9.39), we see that this expression becomes

$$
\operatorname{Vol}\left(\Gamma_{\mathrm{E} 3} \cap \Gamma_{\mathrm{E} 3}\right) \stackrel{9.39}{\longrightarrow} \quad-2 \sqrt{\tau_{\mathrm{E} 3}}
$$

Employing now this result in the formula for the F-term potential above, we arrive at

$$
\begin{gathered}
V_{F} \simeq \frac{g_{s}}{2} \varrho\left[8 a_{\mathrm{E} 3}^{2}\left|\mathcal{A}_{\mathrm{E} 3}\right|^{2} \frac{\sqrt{\hat{\tau}_{\mathrm{E} 3}}}{\hat{\mathcal{V}}} e^{-2 a_{\mathrm{E} 3} \hat{\tau}_{\mathrm{E} 3}}-4 a_{\mathrm{E} 3}\left|\mathcal{A}_{\mathrm{E} 3}\right|\left|W_{0}\right| \frac{\hat{\tau}_{\mathrm{E} 3}}{\hat{\mathcal{V}}^{2}} e^{-a_{\mathrm{E} 3} \hat{\tau}_{\mathrm{E} 3}}\right. \\
\left.+\frac{3}{4} \frac{\hat{\xi}}{\hat{\mathcal{V}}^{3}}\left|W_{0}\right|^{2}\right] .
\end{gathered}
$$

By comparing with equation 6.42 from section 6.2, we see that the potential (9.44) is precisely of this form with

$$
\lambda=8 \text {. }
$$

Therefore, utilising formulas 6.46), we see that the F-term potential (9.44) has a minimum at

$$
\left.\hat{\tau}_{\mathrm{E} 3}\right|_{\text {min. }} \simeq\left(\frac{3 \hat{\xi}}{2}\right)^{2 / 3},\left.\quad \hat{\mathcal{V}}\right|_{\text {min. }} \simeq \frac{\left|W_{0}\right| \sqrt{\hat{\tau}_{\mathrm{E} 3}}}{2 a_{\mathrm{E} 3}\left|\mathcal{A}_{\mathrm{E} 3}\right|} e^{a_{\mathrm{E} 3} \hat{\tau}_{\mathrm{E} 3}}
$$

\section{Numerical analysis}

In order to explicitly verify the large volume minimum of the full scalar potential, let us choose some specific values for the parameters in our model. In particular, in the following we will use

$$
a_{\mathrm{E} 3}=2 \pi, \quad\left|\mathcal{A}_{\mathrm{E} 3}\right|=1, \quad\left|W_{0}\right|=5, \quad \varrho=1 .
$$

Employing then also the result for the Euler characteristic (9.13), we compute with the help of 3.22

$$
\xi \simeq 0.3489,
$$


so that the full scalar potential, before the D-term constraint (9.39) has been used, reads

$$
\begin{aligned}
V_{F+D}\left(\mathcal{V}, \tau_{b}, \tau_{c}\right)= & +37.2 g_{s}^{2} \frac{\sqrt{5 \tau_{b}}+\sqrt{\tau_{c}}}{\mathcal{V}} e^{-\frac{4 \pi}{3 g_{s}}\left(\tau_{b}+\tau_{c}\right)} \\
& -20.9 g_{s}^{3} \frac{\tau_{b}+\tau_{c}}{\mathcal{V}^{2}} e^{-\frac{2 \pi}{3 g_{s}}\left(\tau_{b}+\tau_{c}\right)} \\
& +3.3 g_{s}^{4} \frac{1}{\mathcal{V}^{3}} \\
& +166.7 g_{s}^{3} \frac{\left(\sqrt{\tau_{b}}-\sqrt{5 \tau_{c}}\right)^{2}}{\tau_{b}-2 \tau_{c}} \frac{1}{\mathcal{V}^{2}}
\end{aligned}
$$

where all quantities have been expressed in string frame and where for the D-term potential we have chosen the number of branes in the stack $A$ as $N_{A}=5$. Note that we have not yet fixed the value of $g_{s}$ which is determined by the VEV of the dilaton. We have assumed that it is stabilised by fluxes and since we did not perform an explicit analysis of this mechanism, we choose this parameter as

$$
g_{s}=\frac{1}{10} .
$$

Coming back to the potential above, we observe that the dominant part of 9.49 is given by the $\mathrm{D}$-term potential fixing the combination $\tau_{b}=5 \tau_{c}$. On top of that direction, we find a minimum of the potential in the variables $\mathcal{V}$ and $\tau_{b}$. In figures 9.1 and 9.2 , we have plotted two sections through the parameter space showing the potential in the vicinity of the minimum. The numerical values (in string units) in the minimum are

$$
\left.\mathcal{V}\right|_{\text {min. }} \simeq 1.6 \times 10^{16},\left.\quad \quad \tau_{b}\right|_{\text {min. }} \simeq 1.65,\left.\quad \tau_{c}\right|_{\text {min. }} \simeq 0.33 .
$$

For the volume of the Standard Model cycles we find

$$
\left.\tau_{A}\right|_{\text {min. }}=\left.\tau_{B}\right|_{\text {min. }} \simeq 0.33
$$

and the value of the scalar potential in the minimum is

$$
V_{\text {min. }} \simeq-8.8 \times 10^{-55} M_{\mathrm{Pl}}^{4}
$$

\subsubsection{Discussion}

To conclude this section about the LARGE Volume Scenario for the Calabi-Yau manifold $\mathbb{P}_{[1,3,3,3,5]}[15]$, let us briefly discuss and summarise our model. 


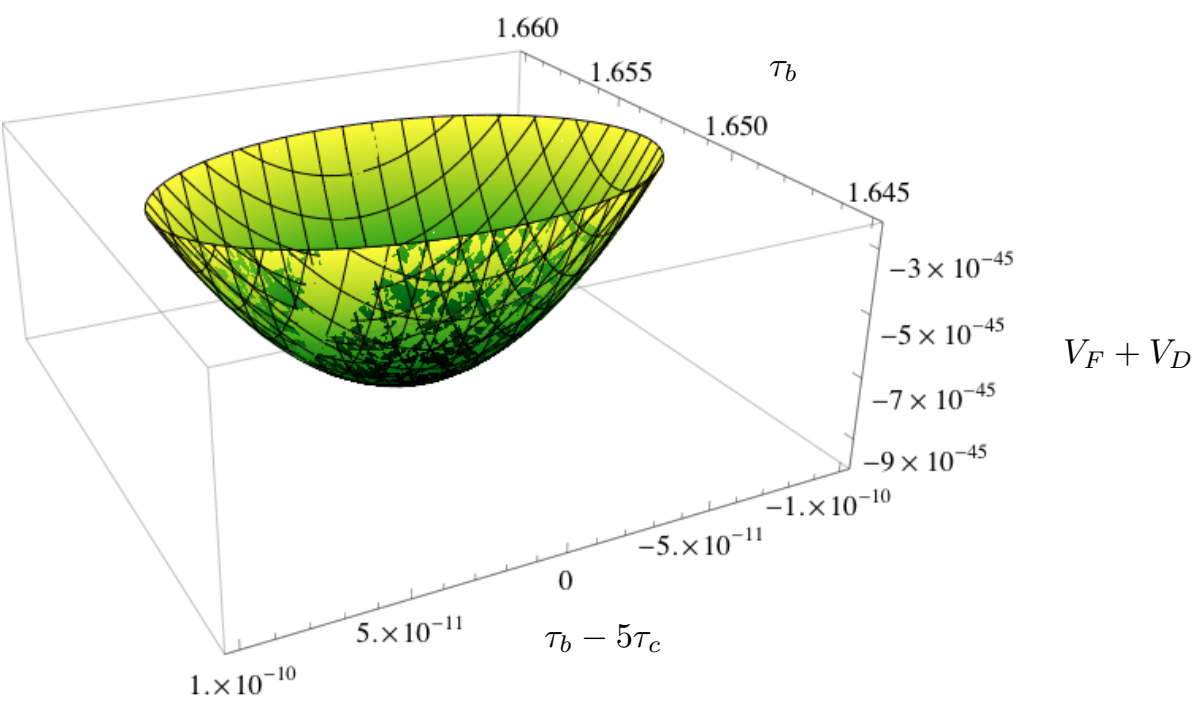

Figure 9.1: The potential $V\left(\mathcal{V}, \tau_{b}, \tau_{c}\right)$ for $\mathcal{V}=1.6 \times 10^{16}$

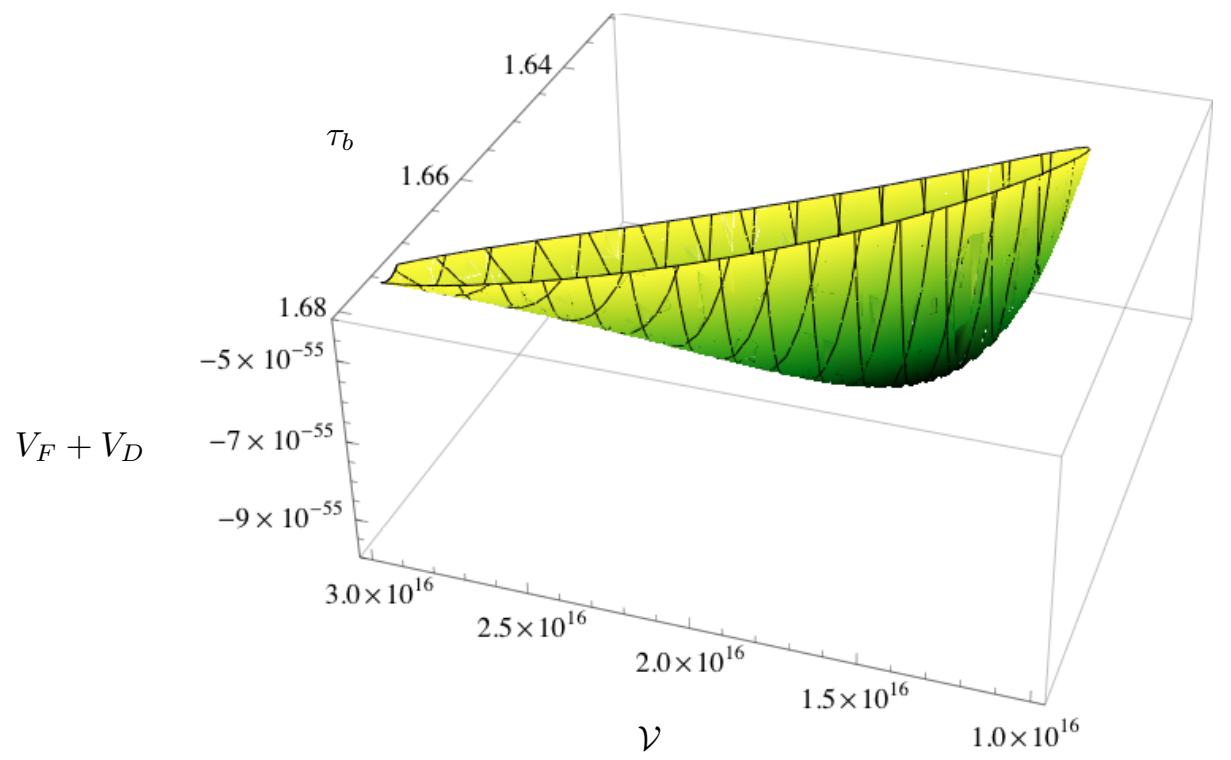

Figure 9.2: The potential $V\left(\mathcal{V}, \tau_{b}, \tau_{c}\right)$ for $\tau_{c}=0.33$. 
- First, we have seen that via the combined D- and F-term potential indeed all Kähler moduli can been stabilised. The reason for considering also the D-term potential was that we explicitly engineered an open string sector in terms of D7-branes with gauge flux, which gave rise to the chiral zero-mode constraint presented in section 8.2 as well as to a generically non-vanishing D-term.

The model presented here serves as an explicit example for the interplay between the open and the closed string sector in the context of moduli stabilisation.

- The next step in the discussion of our model would be to introduce an orientifold projection and to solve the tadpole cancellation conditions. This program has been carried out in the original paper [52] to which we refer for further details.

- As we just mentioned, in principle it is possible to stabilise all Kähler moduli via the combined D- and F-term potential. However, to be more precise, let us recall that

- $\operatorname{Re}\left(T_{\mathrm{b}}\right) \leftrightarrow \mathcal{V}^{2 / 3}$ is stabilised via perturbative effects in the F-term potential, but $\operatorname{Im}\left(T_{\mathrm{b}}\right)$ may be stabilised for instance via non-perturbative effects on the large cycle.

- One linear combination of moduli $t^{I} \leftrightarrow \tau_{I}$ is stabilised through the Dterm potential, while the corresponding axionic partner acquires a mass via the Green-Schwarz mechanism. In particular, as it has been explained in section 7.2.4, the mass matrix (7.55) for the $U(1)$ gauge fields in the present case is proportional to $\mathcal{M}_{a I}$ and so the linear combination of $\operatorname{Im}\left(T_{I}\right) \notin \operatorname{ker}\left(\mathcal{M}_{a I}\right)$ becomes massive.

- The linear combination of moduli $\tau_{\mathrm{E} 3}$ has been stabilised via non-perturbative effects in the F-term potential together with the corresponding axion.

- In case not all small Kähler moduli can be fixed by non-perturbative effects and D-terms, there also exists the possibility that they are frozen similar to $\mathcal{V}$ by perturbative corrections to the F-term potential $[89,92]$. Arguments have been given in [154] that this should occur for the QCD axion.

- As we conjectured in section 9.1, one Kähler modulus has been fixed at the boundary of the Kähler cone implying that the four-cycle $\Gamma_{2}$ has vanishing volume. To resolve this issue, let us note that near the boundary of the Kähler cone String Theory effects such as world-sheet instantons corrections become important which may change this result. Another possibility would be to have a non-vanishing VEV for the fields $\Phi^{\text {abel }}$ introduced on page 123 
which fixes the Fayet-Iliopoulos term at a non-zero value.

- Furthermore, in our model we have computed the chiral charged instanton zero-modes. In addition, one also has to determine the vector-like modes and ensure that the instanton indeed contributes to the non-perturbative superpotential.

- Recalling (9.51), we see that the moduli $\tau_{b}$ and $\tau_{c}$ have been fixed at very small values. This situation is clearly not acceptable since in this regime genuine String Theory effects are important and the supergravity approximation we are employing is not applicable.

Let us investigate this point further. From equation $(9.46)$ and $(3.22)$ we can determine the approximate value of the stabilised four-cycle volume $\tau_{\mathrm{E} 3}$ (in string frame) as

$$
\left.\tau_{\mathrm{E} 3}\right|_{\text {min. }} \simeq\left(\frac{3}{2} \frac{\zeta(3)}{2(2 \pi)^{3}}\right)^{2 / 3}(-\chi(\mathcal{X}))^{2 / 3} \simeq 2.4 \cdot 10^{-2}(-\chi(\mathcal{X}))^{2 / 3}
$$

Therefore, using $\chi(\mathcal{X})=-144$ for our situation, we see that the small value for $\tau_{\mathrm{E} 3}$ is due to the small Euler number. Taking different manifolds with larger Euler characteristic would lead to larger values for the $\tau_{I}$.

- Let us finally mention that due to the modified stabilisation procedure in our model, some of the phenomenological results for the original LARGE Volume Scenario $[134,155,57,156,143]$ no longer hold but have to be adjusted.

\subsection{Summary}

To conclude our discussion about moduli stabilisation in type IIB orientifolds with O3- and O7-planes, let us now summarise our findings from chapter 6 and from the study in the present chapter.

- In section 6.1, we have presented the so-called KKLT scenario [55] and we studied this approach to moduli stabilisation on general grounds as well as in a specific example. In particular,

- in a first step the complex structure moduli $U$ and the axio-dilaton $S$ are stabilised via vanishing F-terms $F_{U}=F_{S}=0$ in a supersymmetric way.

- In a second step, now taking into account non-perturbative contributions to the superpotential involving the Kähler moduli $T$, the latter are stabilised again in a supersymmetric way via vanishing F-terms $F_{T}=0$. 
- Finally, using for instance anti-D3-branes in a Klebanov-Strassler warped throat, the supersymmetric AdS minimum can be uplifted to a non-supersymmetric dS minimum.

However, although the KKLT construction is a crucial step towards the stabilisation of all moduli, this scenario features certain drawbacks. Some of these, as mentioned at the end of section 6.1, are the following

- The two-step procedure of first stabilising $U$ and $S$ by ignoring nonperturbative effects, and in a second step stabilising the Kähler moduli via the latter is ambiguous.

- In order to work in the supergravity approximation, the value of the flux superpotential $W_{0}$ in the minimum has to be fine-tuned.

- Furthermore, $\alpha^{\prime}$-corrections to the Kähler potential have been neglected leading to a no-scale structure of the F-term potential. Taking into account those corrections, the no-scale property is lost and the stabilisation procedure becomes more involved.

- In order to resolve some of the issues of the KKLT Scenario, in section 6.2 we have considered the LARGE Volume Scenario [56,57]. In this approach, the compactification manifold is assumed to be of swiss-cheese type, $\alpha^{\prime}$-corrections are taken into account explicitly and the stabilised volume $\mathcal{V}$ is assumed to be very large.

- These assumptions allow one to perform and $1 / \mathcal{V}$ expansion of the $\mathrm{F}$ term potential. At leading order, this potential is again minimised by vanishing F-terms $F_{U}=F_{S}=0$ originating from the Gukov-Vafa-Witten superpotential.

- At subleading order in $1 / \mathcal{V}$, the complex structure moduli $U$ and the axiodilaton $S$ are treated as fixed and the Kähler moduli $T$ are stabilised via non-perturbative effects.

More concretely, due to the swiss-cheese structure, the big modulus corresponding to the overall volume $\mathcal{V}$ is stabilised via perturbative effects at an exponentially large value while the small Kähler moduli are fixed via non-perturbative contributions to the superpotential. Note that since no vanishing of the F-terms $F_{T}$ is imposed, the resulting AdS minimum in general is non-supersymmetric.

Now, in the LARGE Volume Scenario some issues of the KKLT construction are avoided. For instance, 
- due to the large volume expansion in powers of $1 / \mathcal{V}$, it is possible to first stabilise the complex structure moduli and the axio-dilaton via the flux superpotential, and then stabilise the Kähler moduli via non-perturbative effects in a controlled way.

- Next, the value of the flux superpotential in the minimum $W_{0}$ does not need to be fine-tuned. In fact, in our example we have chosen $W_{0}$ to be of order one.

- Also, we have taken into account $\alpha^{\prime}$-corrections to the superpotential explicitly which were crucial for the moduli stabilisation procedure. In this way, the LVS is stable against such corrections.

Furthermore, in $[91,92]$ it has been shown that the LARGE Volume Scenario is also stable against string-loop corrections to the Kähler potential due to a second no-scale structure.

Finally, the LARGE Volume Scenario has very appealing features in view of phenomenology. We have only touched upon this point at the end of section 6.2 since this part of this work is more concerned with the stabilisation of moduli. For further details in this direction we would like to refer to $[134,155$, $57,156,143]$.

- We now turn to the results obtained in the present chapter. In particular, in the analysis just summarised we have not taken into account D-branes realising the MSSM or some extension thereof. However, as we have explained in detail in section 8.2 , such a sector severely restricts the appearing terms in the non-perturbative superpotential. More concretely, assuming the MSSM to be realised by D7-branes,

- in the non-perturbative superpotential only those (linear combinations) of Kähler moduli can appear which are in the kernel of the matrix defined in (8.18). Since in the presence of chiral MSSM matter this matrix is nontrivial, not all Kähler moduli can appear in the superpotential and are thus not stabilised in the usual way.

This is clearly a problem for phenomenology since additional massless fields in the low energy theory are not in agreement with experiment.

- However, in the presence of D-branes generically also the D-term potential is non-vanishing. In particular, although a detailled analysis is necessary, the vanishing of the Fayet-Iliopoulos term in the D-term potential allows to fix the remaining Kähler moduli.

Therefore, also in the presence of an MSSM sector in general all moduli can be stabilised. But, the moduli stabilisation procedure is different from the 
usual philosophy since now a combination of the D- and F-term potential has to be employed.

In section 9.2 of the present chapter, we have presented a concrete example for a LARGE Volume Scenario where this program has been shown to be possible. In particular,

- we have analysed the specific swiss-cheese manifold $\mathbb{P}_{[1,3,3,3,5]}[15]$ with three Kähler moduli,

- we have illustrated how to satisfy the chiral zero-mode constraint as well as the vanishing of the D-term via a specific combination of D7-branes with gauge flux,

- we have investigated the resulting F-term potential and argued that a large volume minimum can be obtained,

- and we verified the stabilisation procedure numerically for the combined D- and F-term potential.

The example we have presented shows that indeed all Kähler moduli can be stabilised, but we have also seen that there is an complex interplay between the open and closed string sector which modifies the usual moduli stabilisation scenarios. 


\section{Part IV}

\section{Summary and Outlook}





\section{Chapter 10}

\section{Summary and Outlook}

Let us finish this thesis with a summary of the topics discussed in this work as well as with an outlook on further directions of research. However, since at the end of each of the chapters in part III we have already provided a detailled summary of the three individual topics

- Model Building Constraints for Type IIB Orientifolds with O3- and O7-Planes,

- D-Brane Instantons and

- Moduli Stabilisation in the LARGE Volume Scenario,

we will not repeat these summaries again but rather emphasise the interplay of the three subjects. To do so, we will employ the illustration shown on page 21 in the introduction.

\subsection{Summary}

In particular, in figure 10.1 we have recalled the illustration from section 1.3 which we now want to discuss in some detail using the results obtained in this work.

- Let us start with chapter 3 and corresponding appendix B, where we have studied the Kähler potential for compactifications of type IIB supergravity on Calabi-Yau three-folds together with the resulting Kähler metric. In this chapter, we have also introduced and motivated the Gukov-Vafa-Witten superpotential describing flux compactifications.

We turn to figure 10.1 .

(1) Consider first the arrow marked by (1). In section 7.2.4, we have seen that the Kähler potential $\mathcal{K}$ (or rather its first derivative with respect 


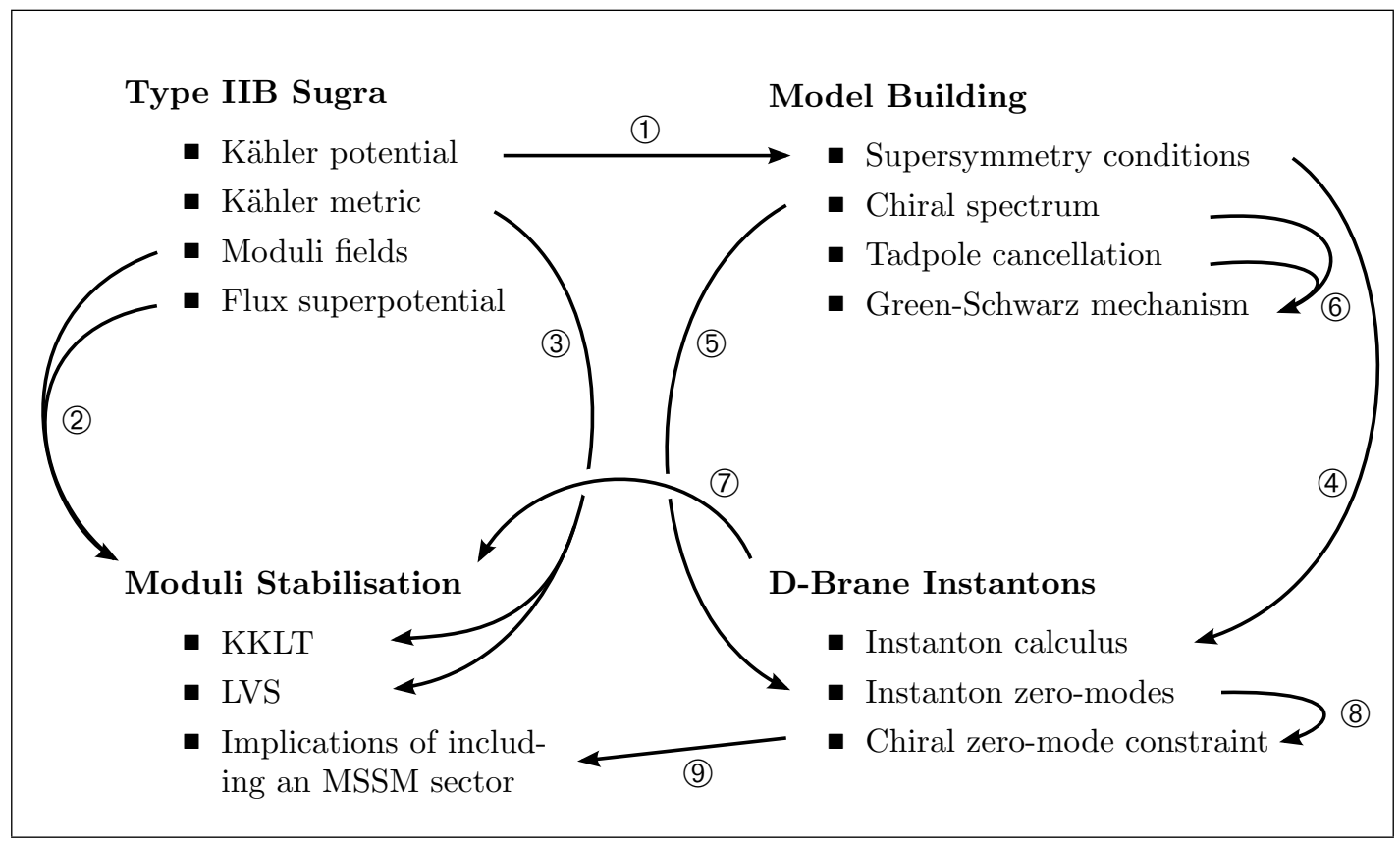

Figure 10.1: Overview on the interplay between the topics discussed in this work.

to the moduli fields) is used for the computation of the Fayet-Iliopoulos parameter. In the absence of matter fields with non-zero vacuum expectation value, the vanishing of the FI term then resembles (part of) the supersymmetry conditions for D-branes.

(2) Next, turning to arrows (2), in chapters 6 and 9 we have seen that the Gukov-Vafa-Witten superpotential introduced in section 3.2.3 is crucial for the stabilisation of the complex structure moduli $U$ and the axiodilaton $S$. In particular, for a general choice of background flux, this superpotential generically allows to stabilise all moduli $U$ and $S$.

(3) Furthermore, in order to discuss moduli stabilisation, the form of the Kähler metric is important. For the KKLT scenario introduced in section 6.1 we have deduced a no-scale property using the explicit form of equation 3.25) derived in chapter 3. In addition, the $\alpha^{\prime}$-corrections to the Kähler metric explicitly computed in appendix B where crucial to realise the LARGE Volume Scenario as explained in section 6.2 .

- Next, we turn to chapters 4 and 7 where we have discussed model building constraints for type IIB orientifolds with O3- and O7-planes. In particular, we have investigated the rules for computing the chiral spectrum, we have explained the supersymmetry conditions for D7-branes with gauge flux as 
well as the Freed-Witten anomaly cancellation condition and the K-theory constraints. We have also determined the tadpole cancellation conditions including the general case with $h_{-}^{1,1} \neq 0$ - and we have studied in detail the generalised Green-Schwarz mechanism.

We turn again to figure 10.1

(4) The supersymmetry conditions explained in section 4.4 are not only important for D7-brane configurations but are also crucial for the D-brane instanton calculus developed in section 5.2. In particular, similar conditions as for the D7-branes apply to E3-brane instantons in order to be supersymmetric.

(5) Furthermore, the rules for computing the (chiral) spectrum introduced in section 4.2 are also applicable when determining the instanton zeromodes. The latter are again a crucial point in the D-brane instanton calculus.

(6) The rules for computing the chiral spectrum have been employed in section 7.2.1 for determining the chiral anomalies; and the tadpole cancellation conditions, an important constraint for D-brane model building, have been derived in section 7.1. The latter are crucial for the cancellation of chiral anomalies via the generalised Green-Schwarz mechanism, and we have explicitly verified this mechanism for type IIB orientifolds with O3- and O7-planes in section 7.2 .

- In chapter 5, we have introduced the D-brane instanton calculus, and in chapter 8 we have used this formalism to derive the Affleck-Dine-Seiberg superpotential in the context of String Theory as well as to derive a "chiral zero-mode constraint" in the presence of an MSSM sector.

(7) As explained in detail in chapter 5, D-brane instantons can contribute to the non-perturbative superpotential. In particular, in the context of type IIB orientifolds with O3- and O7-planes, E3-brane instantons give rise to an exponential dependence of the superpotential on the Kähler moduli $T$. In the KKLT and LARGE Volume Scenario discussed in section 6.1 and 6.2 respectively, such terms are crucial to stabilise the moduli $T$.

(8) Also, the knowledge about instanton zero-modes presented in section 5.1 has been employed in section 8.2 in the development of the "chiral zero-mode constraint". This constraint restricts the appearing terms in the non-perturbative superpotential when an MSSM sector realised by D-branes is taken into account.

(9) Finally, as an important result of this thesis, in chapter 9 the chiral zeromode constraint has been applied to the LARGE Volume Scenario im- 
plying that not all Kähler moduli can be stabilised via non-perturbative effects if an MSSM sector is incorporated. However, as explained in detail in section 9.1, the D-term potential provides additional terms in the scalar potential which generically allow to stabilise the remaining Kähler moduli.

- To finish our discussion of figure 10.1, we recall that in chapter 6 we have explained moduli stabilisation in the KKLT and LARGE Volume Scenario. Furthermore, in chapter 9 we have taken into account the chiral zero-mode constraint and have constructed a large volume model realising this constraint as well as the stabilisation of Kähler moduli via the D-term potential.

To conclude, we have seen that there is an complex interplay with many interrelations between the three main topics discussed in this work. In order uncover these connections, a detailled study of each of the individual subjects was necessary and a combination thereof allowed us to obtain new results such as the chiral zero-mode constraint.

\subsection{Outlook}

Let us also recall some points which we observed during the course of this work which may be worth to study in the future.

- In the context of model building with type IIB orientifolds, we have seen that the D3-brane tadpole cancellation condition is closely related to a constraint in F-theory. In general, type IIB orientifolds with O3- and O7-planes allow for a study via F-theory where the latter provides additional information about nonperturbative effects usually not captured in the type II description. Therefore, a more detailled study of F-theory models in view of D-brane model building can give us new insight for the type IIB constructions.

- At some instances, we have already mentioned that the common analysis concerning moduli in type IIB orientifolds with O3- and O7-planes does not take into account the $\hat{\mathcal{A}}$-terms in the Chern-Simons action $(7.4)$ which encode gravitational corrections. These are expected to have implications on the Dbrane instanton calculus and may suggest that the also the Dirac-Born-Infeld action for D-branes should include gravitational corrections.

Very much related to this point is our observation made in equation (7.91) about the structure of $\alpha^{\prime}$-corrections to the Fayet-Iliopoulos terms for D3-, D5-, D7- and D9-branes. In particular, for the D9-brane FI term the cor- 
rections to the Kähler potential fit nicely with $\hat{\mathcal{A}}$-term of the Chern-Simons action, and we expect a similar structure also for the lower-dimensional branes.

- In view of moduli stabilisation, it would be interesting to further investigate the case $h_{-}^{1,1} \neq 0$ in which the $G^{\hat{\imath}}$-moduli are present. Since instanton corrections to the superpotential involving $G^{\hat{\imath}}$ are restricted, the stabilisation of these moduli is different from the mechanism employed for the Kähler moduli.

- Finally, as we have seen in chapter 9, when stabilising Kähler moduli via the Dterm potential, generically these are fixed at the boundary of the Kähler cone. This situation changes for instance the computation of soft supersymmetry breaking terms and thus it is necessary to reconsider these calculations. 


\section{Part V}

\section{Appendices}





\section{Appendix A}

\section{Topological Quantities}

In this appendix, we provide the definition of the quantities used in the ChernSimons actions 2.31) for D-branes and O-planes, and give some details of the calculation leading to the expressions in (7.5).

\section{Definitions}

We start with the definitions (for more details see for instance [59]). The Chern character of a complex vector bundle $F$ is defined in the following way

$$
\operatorname{ch}(F)=\sum_{n=0}^{\infty} \operatorname{ch}_{n}(F), \quad \operatorname{ch}_{n}(F)=\frac{1}{n !} \operatorname{tr}\left[\left(\frac{i F}{2 \pi}\right)^{n}\right]
$$

where the trace is over the fundamental representation. The Chern character satisfies

$$
\operatorname{ch}(E \oplus F)=\operatorname{ch}(E)+\operatorname{ch}(F) .
$$

The $\hat{\mathcal{A}}$-genus and the Hirzebruch $\mathcal{L}$-polynomial can be expressed in terms of the Pontrjagin classes $p_{i}$ as

$$
\begin{aligned}
& \hat{\mathcal{A}}(F)=1-\frac{1}{24} p_{1}+\frac{1}{5760}\left(7 p_{1}^{2}-4 p_{2}\right)+\ldots \\
& \mathcal{L}(F)=1+\frac{1}{3} p_{1}+\frac{1}{45}\left(-p_{1}^{2}+7 p_{2}\right)+\ldots
\end{aligned}
$$

and satisfy

$$
\hat{\mathcal{A}}(E \oplus F)=\hat{\mathcal{A}}(E) \wedge \hat{\mathcal{A}}(F), \quad \mathcal{L}(E \oplus F)=\mathcal{L}(E) \wedge \mathcal{L}(F) .
$$


For the following, we will only need the definition of the first Pontrjagin class of a real vector bundle which reads

$$
p_{1}(F)=-\frac{1}{2} \operatorname{tr}\left[\left(\frac{F}{2 \pi}\right)^{2}\right],
$$

where the trace is again over the fundamental representation. If the real $2 k$ dimensional bundle $F_{\mathbb{R}}$ can be written as a complex $k$-dimensional bundle $F_{\mathbb{C}}$, we have the relation

$$
p_{1}\left(F_{\mathbb{R}}\right)=\left[c_{1}\left(F_{\mathbb{C}}\right)\right]^{2}-2 c_{2}\left(F_{\mathbb{C}}\right),
$$

where $c_{1}$ and $c_{2}$ denote the first and second Chern class expressed as

$$
c_{1}(F)=\operatorname{ch}_{1}(F), \quad c_{2}(F)=\frac{1}{2}\left[\operatorname{ch}_{1}(F)\right]^{2}-\operatorname{ch}_{2}(F) .
$$

\section{Calculation leading to 7.5}

After stating these definitions and relations, let us concentrate on a complex twodimensional holomorphic submanifold $\Gamma$ of a complex three-dimensional CalabiYau manifold $\mathcal{X}$. Since the first Chern class of a Calabi-Yau manifold vanishes, we find

$$
0=c_{1}\left(T_{\mathcal{X}}\right)=\operatorname{ch}_{1}\left(T_{\Gamma} \oplus N_{\Gamma}\right)=\operatorname{ch}_{1}\left(T_{\Gamma}\right)+\operatorname{ch}_{1}\left(N_{\Gamma}\right)=c_{1}\left(T_{\Gamma}\right)+c_{1}\left(N_{\Gamma}\right),
$$

where $T$ denotes the tangential bundle and $N$ the normal bundle. Noting then that the second Chern class of a line bundle such as $N_{\Gamma}$ vanishes, we calculate using (A.6) and (A.8)

$$
p_{1}\left(T_{\Gamma}\right)-p_{1}\left(N_{\Gamma}\right)=\left[c_{1}\left(T_{\Gamma}\right)\right]^{2}-2 c_{2}\left(T_{\Gamma}\right)-\left[c_{1}\left(N_{\Gamma}\right)\right]^{2}+2 c_{2}\left(N_{\Gamma}\right)=-2 c_{2}\left(T_{\Gamma}\right)
$$

where we interpreted the real vector bundles as complex ones. This computation allows us now to write the $\hat{\mathcal{A}}$-terms in the Chern-Simons action more feasible. The square root as well as the inverse of the $\hat{\mathcal{A}}$-genus are understood as a series expansion and using (A.4), we find

$$
\begin{aligned}
& \sqrt{\frac{\hat{\mathcal{A}}\left(\mathcal{R}_{T}\right)}{\hat{\mathcal{A}}\left(\mathcal{R}_{N}\right)}}=\sqrt{\hat{\mathcal{A}}\left(\mathcal{R}^{(4)}\right)} \wedge \sqrt{\frac{\hat{\mathcal{A}}\left(\mathcal{R}_{T}^{(6)}\right)}{\hat{\mathcal{A}}\left(\mathcal{R}_{N}^{(6)}\right)}} \\
= & \left(1-\frac{1}{48} p_{1}\left(\mathcal{R}^{(4)}\right)+\ldots\right) \wedge\left(1-\frac{1}{48} p_{1}\left(\mathcal{R}_{T}^{(6)}\right)+\frac{1}{48} p_{1}\left(\mathcal{R}_{N}^{(6)}\right)+\ldots\right) \\
= & \left(1+\frac{1}{96}\left(\frac{l_{s}^{2}}{2 \pi}\right)^{2} \operatorname{tr}\left(R^{2}\right)+\ldots\right) \wedge\left(1+\frac{l_{s}^{4}}{24} c_{2}(\Gamma)+\ldots\right),
\end{aligned}
$$


where $^{(4)}$ denotes the four-dimensional and ${ }^{(6)}$ the internal part of $\mathcal{R}$. In going from the second to the third line, we employed our definition (2.28) and we adjusted our notation as

$$
\begin{aligned}
& p_{1}\left(\mathcal{R}_{T}^{(6)}\right)=l_{s}^{4} p_{1}\left(\bar{R}_{T}\right)=l_{s}^{4} p_{1}\left(T_{\Gamma}\right), \quad c_{2}\left(T_{\Gamma}\right)=c_{2}(\Gamma), \\
& p_{1}\left(\mathcal{R}_{N}^{(6)}\right)=l_{s}^{4} p_{1}\left(\bar{R}_{N}\right)=l_{s}^{4} p_{1}\left(N_{\Gamma}\right) .
\end{aligned}
$$

Along the same lines, we obtain for the Hirzebruch $\mathcal{L}$-polynomial the following result

$$
\sqrt{\frac{\mathcal{L}\left(\mathcal{R}_{T} / 4\right)}{\mathcal{L}\left(\mathcal{R}_{N} / 4\right)}}=\left(1-\frac{1}{192}\left(\frac{l_{s}^{2}}{2 \pi}\right)^{2} \operatorname{tr}\left(R^{2}\right)+\ldots\right) \wedge\left(1-\frac{l_{s}^{4}}{48} c_{2}(\Gamma)+\ldots\right) .
$$




\section{Appendix B}

\section{Details on the Kähler Metric}

In this appendix, we present some details of the computation of the Kähler metric involving $T_{i}$ and $S$ derived from the Kähler potential (3.19). Let us recall this expression for convenience

$$
\mathcal{K}=-\log (S+\bar{S})-2 \log \left(\hat{\mathcal{V}}+\frac{\hat{\xi}}{2}\right)-\log \left(-\frac{i}{l_{s}^{6}} \int_{\mathcal{X}} \Omega_{3} \wedge \bar{\Omega}_{3}\right)
$$

Note furthermore, we assume $h_{-}^{1,1}=0$ so that $\mathcal{V}$ does not depend on $G^{\hat{\imath}}$ or $S$.

\section{Prerequisites}

- First, let us recall and refine the definitions given in equations (2.14 - 2.16). Using $\rho_{i}=\frac{1}{l_{s}^{4}} \int_{\gamma_{i}} C_{4}$, in the present case we can write

$$
T_{i}=\hat{\tau}_{i}+i \rho_{i}, \quad S=e^{-\phi}-i C_{0} .
$$

- We furthermore recall that $J=t^{i} \omega_{i}$ as well as the definition of the overall volume of $\mathcal{X}$ and the four-cycle volumes of the internal space

$$
\mathcal{V}=\frac{1}{6} k_{i j k} t^{i} t^{j} t^{k}, \quad \tau_{i}=\frac{1}{2} k_{i j k} t^{j} t^{k} .
$$

- With ${ }^{-1}$ denoting the matrix inverse, three useful relations read as follows

$$
k_{i j k} t^{k}=\frac{1}{2}\left(\frac{\partial^{2} \mathcal{V}}{\partial \tau_{i} \partial \tau_{j}}\right)^{-1}, \quad \frac{t^{i}}{2}=\frac{\partial \mathcal{V}}{\partial \tau_{i}}, \quad \tau_{i} t^{i}=3 \mathcal{V}
$$


- With $g_{s}=e^{\phi}$ the string-coupling and $\chi(\mathcal{X})$ the Euler number of the internal space, the $\alpha^{\prime}$-correction takes the following form

$$
\hat{\xi}=\frac{\xi}{g_{s}^{3 / 2}}=\xi\left(\frac{S+\bar{S}}{2}\right)^{\frac{3}{2}}, \quad \xi=-\frac{\zeta(3) \chi(\mathcal{X})}{2(2 \pi)^{3}} .
$$

\section{Kähler Vectors}

The first derivatives of the Kähler potential (B.1) with respect to $T_{i}$ and $S$ read

$$
\mathcal{K}_{i}=\frac{\partial \mathcal{K}}{\partial T_{i}}=-\frac{1}{2} \frac{\hat{t}^{i}}{\hat{\mathcal{V}}+\frac{\hat{\xi}}{2}}, \quad \quad \mathcal{K}_{S}=\frac{\partial \mathcal{K}}{\partial S}=-\frac{1}{S+\bar{S}} \frac{\hat{\mathcal{V}}+2 \hat{\xi}}{\hat{\mathcal{V}}+\frac{\hat{\xi}}{2}}
$$

\section{Kähler Metric}

The Kähler metric computed from (B.1) reads

$$
\begin{aligned}
& G_{i \bar{j}}=\frac{\partial^{2} \mathcal{K}}{\partial T_{i} \partial \bar{T}_{j}}=-\frac{1}{4} \frac{1}{\hat{\mathcal{V}}+\frac{\hat{\xi}}{2}}\left(\left(k_{i j k} \hat{t}^{k}\right)^{-1}-\frac{1}{2} \frac{\hat{t}^{i} \hat{t}^{j}}{\hat{\mathcal{V}}+\frac{\hat{\xi}}{2}}\right) \\
& G_{i \bar{S}}=\frac{\partial^{2} \mathcal{K}}{\partial T_{i} \partial \bar{S}}=\frac{3}{8} \frac{\hat{t}^{i}}{S+\bar{S}} \frac{\hat{\xi}}{\left(\hat{\mathcal{V}}+\frac{\hat{\xi}}{2}\right)^{2}}, \\
& G_{S \bar{S}}=\frac{\partial^{2} \mathcal{K}}{\partial S \partial \bar{S}}=\frac{1}{(S+\bar{S})^{2}} \frac{4 \hat{\mathcal{V}}^{2}+\hat{\mathcal{V}} \hat{\xi}+4 \hat{\xi}^{2}}{(2 \hat{\mathcal{V}}+\hat{\xi})^{2}}
\end{aligned}
$$

\section{Inverse Kähler Metric}

The inverse Kähler metric can be determined to be of the following form

$$
\begin{aligned}
& G^{i \bar{j}}=-4\left(\hat{\mathcal{V}}+\frac{\hat{\xi}}{2}\right) k_{i j k} \hat{t}^{k}+\hat{\tau}_{i} \hat{\tau}_{j} \frac{4 \hat{\mathcal{V}}-\hat{\xi}}{\hat{\mathcal{V}}-\hat{\xi}}, \\
& G^{i \bar{S}}=-\frac{3}{4}(S+\bar{S}) \frac{\hat{\xi}}{\hat{\mathcal{V}}+\frac{\hat{\xi}}{2}} \frac{2 \hat{\mathcal{V}}+\hat{\xi}}{\hat{\mathcal{V}}-\hat{\xi}} \hat{\tau}_{i}, \\
& G^{S \bar{S}}=\frac{(S+\bar{S})^{2}}{4} \frac{4 \hat{\mathcal{V}}-\hat{\xi}}{\hat{\mathcal{V}}-\hat{\xi}} .
\end{aligned}
$$


Note that by employing (B.4), the inverse Kähler metric for the Kähler moduli can be written as

$$
G^{i \bar{j}}=-2\left(\hat{\mathcal{V}}+\frac{\hat{\xi}}{2}\right)\left(\frac{\partial^{2} \hat{\mathcal{V}}}{\partial \hat{\tau}_{i} \partial \hat{\tau}_{j}}\right)^{-1}+\hat{\tau}_{i} \hat{\tau}_{j} \frac{4 \hat{\mathcal{V}}-\hat{\xi}}{\hat{\mathcal{V}}-\hat{\xi}}
$$

\section{F-Term Potential}

We finally present the explicit result for the F-term potential when only Kähler moduli are considered. With $i=1, \ldots, h_{+}^{1,1}$ labelling the Kähler moduli $T_{i}{ }^{-1}$ denoting the matrix inverse and a sum over repeated indices understood, we find

$$
\begin{aligned}
& V_{F}=e^{\mathcal{K}}\left(D_{i} W G^{i \bar{j}} D_{\bar{j}} \bar{W}-3|W|^{2}\right) \\
& V_{F}=e^{\mathcal{K}}\left[-2\left(\hat{\mathcal{V}}+\frac{\hat{\xi}}{2}\right)\left(\frac{\partial^{2} \hat{\mathcal{V}}}{\partial \hat{\tau}_{i} \partial \hat{\tau}_{j}}\right)^{-1}\left(\partial_{i} W\right)\left(\partial_{\bar{j}} \bar{W}\right)\right. \\
& +\frac{4 \hat{\mathcal{V}}-\hat{\xi}}{\hat{\mathcal{V}}-\hat{\xi}} \hat{\tau}_{i}\left(\partial_{i} W\right) \hat{\tau}_{j}\left(\partial_{\bar{j}} \bar{W}\right) \\
& -\frac{4 \hat{\mathcal{V}}^{2}+\hat{\mathcal{V}} \hat{\xi}+4 \hat{\xi}^{2}}{(\hat{\mathcal{V}}-\hat{\xi})(2 \hat{\mathcal{V}}+\hat{\xi})}\left(\hat{\tau}_{i}\left(\partial_{i} W\right) \bar{W}+\text { c.c. }\right) \\
& +3 \hat{\xi} \frac{\hat{\mathcal{V}}^{2}+7 \hat{\mathcal{V}} \hat{\xi}+\hat{\xi}^{2}}{(\hat{\mathcal{V}}-\hat{\xi})(2 \hat{\mathcal{V}}+\hat{\xi})^{2}}|W|^{2}
\end{aligned}
$$


B. Details on the Kähler Metric 


\section{Appendix C}

\section{The Gukov-Vafa-Witten Superpotential}

In this appendix, we are going to motivate the expression for the Gukov-VafaWitten superpotential stated in equation (3.27). In particular, we explain how the effective four-dimensional type IIB action is modified when considering background fluxes, and we show that (3.27) indeed reproduces these terms. (For more details, see for instance the appendices of [58] or [60].)

\section{Implication of Background Fluxes for the Effective Type IIB Action}

As we just mentioned, we begin by discussing how the background fluxes (2.17) modify the effective four-dimensional theory. In particular, by replacing $\mathrm{H}_{3} \rightarrow$ $H_{3}+\bar{H}_{3}$ and $F_{3} \rightarrow F_{3}+\bar{F}_{3}$, one finds that (the orientifold projection of) the type IIB action 2.11) contains the additional terms

$$
-\frac{1}{4 \kappa_{10}^{2}} \int\left[e^{-2 \phi} \bar{H}_{3} \wedge \star \bar{H}_{3}+\overline{\widetilde{F}}_{3} \wedge \star \overline{\widetilde{F}}_{3}\right]
$$

where $\overline{\widetilde{F}}_{3}=\bar{F}_{3}-C_{0} \bar{H}_{3}$. Recalling then from equation 2.14 that $\tau=C_{0}+i e^{-\phi}$ and defining the so-called $G_{3}$-flux as

$$
G_{3}=\bar{F}_{3}-\tau \bar{H}_{3}
$$

we can write the expression given in equation (C.1) in the following way

$$
-\frac{1}{4 \kappa_{10}^{2}} \int G_{3} \wedge \star \bar{G}_{3}
$$


Note that here, by abuse of notation, $\bar{G}_{3}$ denotes the complex conjugate of $G_{3}$ while $\bar{F}_{3}$ and $\bar{H}_{3}$ stand for the real fluxes originating from the field strengths $F_{3}$ and $\mathrm{H}_{3}$. Defining finally

$$
G_{3}^{ \pm}=\frac{1}{2}\left(G_{3} \pm i \star_{6} G_{3}\right), \quad \star_{6} G_{3}^{ \pm}=\mp i G_{3}^{ \pm},
$$

where $\star_{6}$ is the Hodge- $\star$-operator for the compactification manifold $\mathcal{X}$, we can write equation C.3 as

$$
-\frac{1}{4 \kappa_{10}^{2}} \int_{\mathbb{R}^{3,1}} \star_{4} 1 \int_{\mathcal{X}}\left(2 G_{3}^{+} \wedge \star_{6} \bar{G}_{3}^{+}-i G_{3} \wedge \bar{G}_{3}\right)
$$

with $\star_{4} 1$ denoting the volume element for the four-dimensional space $\mathbb{R}^{3,1}$.

\section{The Four-Dimensional Scalar Potential}

The second term of C.5 involving $G_{3} \wedge \bar{G}_{3}$ is topological and is cancelled by means of the D3-brane tadpole cancellation condition studied in section 7.1. Let us therefore focus on the first term and go from string to Einstein frame via

$$
g_{M N}^{\text {string }}=e^{\phi / 2} g_{M N}^{\text {Einstein }}, \quad M, N=0, \ldots, 9,
$$

where $\phi$ denotes the dilaton. Carefully working out the factors of $e^{\phi / 2}$ and performing a Weyl rescaling $g_{\mu \nu} \rightarrow \hat{\mathcal{V}} g_{\mu \nu}$ of the four-dimensional metric, we can identify the four-dimensional potential as

$$
V_{\text {flux }}=\frac{1}{2 l_{s}^{6}} \frac{1}{\hat{\mathcal{V}}^{2}} e^{\phi} \int_{\mathcal{X}} G_{3}^{+} \wedge \star_{6} \bar{G}_{3}^{+} .
$$

\section{Re-Writing Equation (C.7)}

Let us now rewrite equation (C.7). To do so, we observe that since $\bar{H}_{3}$ and $\bar{F}_{3}$ are odd under the holomorphic involution $\sigma$, we can expand $G_{3}^{+}$in a basis of $H_{-}^{3}(\mathcal{X})$. We furthermore note that

$$
\star_{6} G_{3}^{+}=-i G_{3}^{+}, \quad \star_{6} \Omega_{3}=-i \Omega_{3}, \quad \star_{6} \bar{\chi}_{\hat{\kappa}}=-i \bar{\chi}_{\hat{\kappa}},
$$

where $\left\{\bar{\chi}_{\hat{\kappa}}\right\}$ is a basis of $H_{\bar{\partial}-}^{1,2}(\mathcal{X})$ defined around equation 3.15, which allows us to expand $G_{3}^{+}$in the basis

$$
\left(\Omega_{3}, \bar{\chi}_{\hat{\kappa}}\right) \quad \in \quad H_{\bar{\partial}-}^{3,0}(\mathcal{X}) \oplus H_{\bar{\partial}-}^{1,2}(\mathcal{X}) .
$$


Observing then that $\int_{\mathcal{X}} \bar{\Omega}_{3} \wedge G_{3}^{+}=\int_{\mathcal{X}} \bar{\Omega}_{3} \wedge G_{3}$ as well as that $\int_{\mathcal{X}} \chi_{\hat{\kappa}} \wedge G_{3}^{+}=$ $\int_{\mathcal{X}} \chi_{\hat{\kappa}} \wedge G_{3}$, we can express $G_{3}^{+}$as

$$
G_{3}^{+}=-\frac{1}{\int_{\mathcal{X}} \Omega_{3} \wedge \bar{\Omega}_{3}}\left(\Omega_{3} \int_{\mathcal{X}} \bar{\Omega}_{3} \wedge G_{3}-G^{U^{\hat{\kappa}} \bar{U}^{\hat{\lambda}}} \bar{\chi}_{\hat{\lambda}} \int_{\mathcal{X}} \chi_{\hat{\kappa}} \wedge G_{3}\right),
$$

where $G^{U^{\hat{\kappa}} \bar{U}^{\hat{\lambda}}}$ denotes the inverse of the Kähler metric for the complex structure moduli defined in equation $(3.26)$. With $\mathcal{M}^{\hat{\kappa} \hat{\lambda}}$ denoting the inverse of (3.18), we compute

$$
\begin{aligned}
V_{\text {flux }} & =\frac{1}{2 l_{s}^{6}} \frac{1}{\hat{\mathcal{V}}^{2}} e^{\phi} \int_{\mathcal{X}} G_{3}^{+} \wedge \star_{6} \bar{G}_{3}^{+} \\
& =-\frac{i}{2 l_{s}^{12}} \frac{e^{\phi}}{\hat{\mathcal{V}}^{2}}\left[\left(\int_{\mathcal{X}} \chi_{\hat{\kappa}} \wedge G_{3}\right) \mathcal{M}^{\hat{\kappa} \hat{\hat{\lambda}}}\left(\int_{\mathcal{X}} \bar{\chi}_{\hat{\kappa}} \wedge \bar{G}_{3}\right)-\frac{\left|\int_{\mathcal{X}} \Omega_{3} \wedge \bar{G}_{3}\right|^{2}}{\frac{1}{l_{s}^{6}} \int_{\mathcal{X}} \Omega_{3} \wedge \bar{\Omega}_{3}}\right] .
\end{aligned}
$$

\section{F-Term Potential via the Gukov-Vafa-Witten Superpotential}

Let us now determine the F-term potential for type IIB orientifold compactifications originating from the Gukov-Vafa-Witten superpotential

$$
W_{\mathrm{GVW}}=\frac{1}{l_{s}^{6}} \int_{\mathcal{X}} \Omega_{3} \wedge G_{3}
$$

More concretely, we restrict ourselves to the case of vanishing $\alpha^{\prime}$-corrections, i.e. $\xi=0$, and use 3.25 to compute

$$
V_{F}=e^{\mathcal{K}}\left(G^{U \bar{U}} F_{U} \bar{F}_{\bar{U}}+G^{S \bar{S}} F_{S} \bar{F}_{\bar{S}}\right) .
$$

Utilising the explicit form of the Kähler potential (3.19) and the expression for the Kähler metrics (3.26), we find

$$
\begin{aligned}
F_{U^{\hat{\kappa}}} & =\frac{1}{l_{s}^{6}} \int_{\mathcal{X}} \chi_{\hat{\kappa}} \wedge G_{3}, & G^{U^{\hat{\kappa}} U^{\bar{\lambda}}} & =-\frac{1}{l_{s}^{6}} \int_{\mathcal{X}} \Omega_{3} \wedge \bar{\Omega}_{3} \mathcal{M}^{\hat{\kappa} \hat{\bar{\lambda}}}, \\
F_{S} & =-\frac{1}{S+\bar{S}} \frac{1}{l_{s}^{6}} \int_{\mathcal{X}} \Omega_{3} \wedge \bar{G}_{3}, & G^{S \bar{S}} & =(S+\bar{S})^{2},
\end{aligned}
$$

where $\mathcal{M}^{\hat{\kappa} \hat{\lambda}}$ denotes the inverse of the matrix defined in equation (3.18). Employing these expressions in (C.13), we arrive at

$$
V_{F}=-\frac{i}{2 l_{s}^{12}} \frac{e^{\phi}}{\hat{\mathcal{V}}^{2}}\left[\left(\int_{\mathcal{X}} \chi_{\hat{\kappa}} \wedge G_{3}\right) \mathcal{M}^{\hat{\hat{\kappa}} \hat{\lambda}}\left(\int_{\mathcal{X}} \bar{\chi}_{\hat{\kappa}} \wedge \bar{G}_{3}\right)-\frac{\left|\int_{\mathcal{X}} \Omega_{3} \wedge \bar{G}_{3}\right|^{2}}{\frac{1}{l_{s}^{6}} \int_{\mathcal{X}} \Omega_{3} \wedge \bar{\Omega}_{3}}\right] \text {. }
$$


By comparing with the potential C.11 obtained from a dimensional reduction of the type IIB action in the presence of background flux, we see that indeed the Gukov-Vafa-Witten superpotential C.12 captures these effects in terms of a four-dimensional $\mathcal{N}=1$ supergravity description. 


\section{Appendix D}

\section{Chiral Anomalies for Gauge Groups $\mathrm{SO}(2 \mathrm{~N})$ and $\mathrm{Sp}(2 \mathrm{~N})$}

In this appendix, we comment on the generalised Green-Schwarz mechanism for the case of gauge groups $S O(2 N)$ and $S p(2 N)$. Since both Lie groups are simple, there are no cubic abelian or mixed abelian-gravitational anomalies for these cases. For the cubic non-abelian anomaly, let us note that the anomaly is proportional to

$$
\mathcal{A}^{a b c}(r)=\frac{1}{2} A(r) d^{a b c}
$$

where $d^{a b c}$ is the unique symmetric invariant. This invariant only exists for $S U(N)$ and $S O(6)$ (which has the same Lie algebra as $S U(4)$ ) and so there is no cubic non-abelian anomaly to be studied in the present case.

For the mixed abelian-non-abelian anomaly, let us note that the dimension and the index for the fundamental representation of both $S O(2 N)$ and $S p(2 N)$ are found to be

$$
\operatorname{dim}(F)=2 N, \quad C(F)=1 .
$$

The anomaly coefficient is then computed as

$$
\mathcal{A}_{U(1)_{a}-S p / S O\left(2 N_{\mathrm{D} 7_{b}}\right)^{2}}=\sum_{F} Q_{a}(F) C_{b}(F)=-N_{\mathrm{D} 7_{a}}\left(I_{a b}-I_{a^{\prime} b}\right),
$$

which is, up to a factor of $\frac{1}{2}$, the same as in 7.31 . For the calculation of the Green-Schwarz diagrams, we note that $C(F)=1$ by definition means

$$
\operatorname{tr}\left(T^{A} T^{B}\right)=\delta^{A B}
$$


which differs from the result for $S U(N)$ by a factor of $\frac{1}{2}$. Using this observation and following the same steps as in the computation for $S U(N)$, one finds that the Green-Schwarz diagrams are precisely of the form (D.3) (up to a common prefactor). Therefore, also for $S O(2 N)$ and $S p(2 N)$ the mixed abelian-non-abelian anomalies are cancelled via the generalised Green-Schwarz mechanism. 


\section{Appendix E}

\section{The Chiral Zero-Mode Constraint for $\mathbf{h}_{-}^{1,1} \neq 0$}

In this appendix, we are going to discuss the chiral zero-mode constraint from section 8.2 for the case of type IIB orientifolds with O3- and O7-planes where $h_{-}^{1,1} \neq 0$.

\section{E1-Instanton Action}

In order to do so, we start by collecting some facts about E1-instantons. Let us consider an E1-instanton without "gauge flux" wrapped on a basis two-cycle $\Sigma^{\hat{\imath}} \in H_{2-}(\mathcal{X}, \mathbb{Z})$ which is odd under the holomorphic involution $\sigma$. Taking into account the calibration condition for a supersymmetric E1-instanton [71]

$$
d^{2} \xi \sqrt{\operatorname{det}\left(\left(\varphi^{*} g^{(6)}\right)_{i j}+\frac{i \overline{\mathcal{F}}_{i j}}{2 \pi}\right)}=e^{-i \theta}\left(J-\frac{\overline{\mathcal{F}}}{2 \pi}\right),
$$

with $\theta=-\frac{\pi}{2}$ and choosing $\kappa_{1}=-1$, we find from (5.2) and (5.3) that

$$
\mathcal{S}_{\mathrm{E} 1}=-2 \pi i G^{\hat{\imath}},
$$

where the moduli $G^{\hat{\imath}}$ had been defined in equation (2.16). As we already know, in the case $h_{-}^{1,1} \neq 0$ in addition to the Kähler moduli also the $G^{\hat{\imath}}$-moduli are present, however, here we see that the latter are in relation with E1-instantons on cycles $\Sigma^{\hat{\imath}}$. 


\section{Chiral Charged Instanton Zero-Modes}

We now reconsider our arguments from section 8.2. We assume again that intersecting D7-branes with gauge flux give rise to chiral matter, that is

$$
I_{a b}=\frac{\mathcal{M}_{a I}}{N_{\mathrm{D} 7_{a}}} m_{b}^{I}-\frac{\mathcal{M}_{b I}}{N_{\mathrm{D} 7_{b}}} m_{a}^{I} \neq 0,
$$

for some D-branes $a$ and $b$. As before, the chiral charged zero-modes between an E3-instanton of type $O(1)$ without gauge flux and a D7-brane are counted by

$$
Z_{\mathrm{D} 7_{a} \mathrm{E} 3}=\mathcal{M}_{a i} m_{\mathrm{E} 3}^{i}
$$

where $i=1, \ldots, h_{+}^{1,1}$ and a summation over repeated indices is understood. Therefore, if we take $h_{+}^{1,1}$ E3-instantons wrapping linearly independent four-cycles without gauge flux,

$$
\operatorname{rk}\left(\mathcal{M}_{a i}\right), \quad i=1, \ldots, h_{+}^{1,1},
$$

of these will have chiral charged zero-modes. Note that there is a slight difference to our discussion from section 8.2. In particular, by arranging (E.5) to vanish, none of the E3-instantons will have chiral charged zero-modes, but it is nevertheless possible to obtain chiral matter between D7-branes via

$$
I_{a b}=\frac{\mathcal{M}_{a \hat{\imath}}}{N_{\mathrm{D} 7_{a}}} m_{b}^{\hat{\imath}}-\frac{\mathcal{M}_{b \hat{\imath}}}{N_{\mathrm{D} 7_{b}}} m_{a}^{\hat{\imath}} \neq 0
$$

with $\hat{\imath}=1, \ldots, h_{-}^{1,1}$. This would clearly change our conclusions for section 8.2 .

However, as we mentioned, in the case $h_{-}^{1,1} \neq 0$ not only Kähler moduli are present but also $G^{\hat{\imath}}$-moduli which are in relation with E1-instantons. Let us therefore consider $h_{-}^{1,1}$ E1-instantons without gauge flux wrapping linearly independent twocycles

$$
\Gamma_{\mathrm{E} 1_{\Lambda}}=m_{\mathrm{E} 1_{\Lambda} \hat{\imath}} \Sigma^{\hat{\imath}} \in H_{2-}(\mathcal{X}, \mathbb{Z})
$$

where a summation over repeated indices is again understood. From equation (7.78) we can infer a formula for counting chiral charged instanton zero-modes between D7-branes and E1-instantons as

$$
Z_{\mathrm{D} 7_{a} \mathrm{E} 1_{\Lambda}}=N_{\mathrm{D} 7_{a}} \int_{\mathcal{X}}\left[\Gamma_{\mathrm{D} 7_{a}}\right] \wedge\left[\Gamma_{\mathrm{E} 1_{\Lambda}}\right]=N_{\mathrm{D} 7_{a}} m_{\mathrm{D} 7_{a}}^{\hat{\imath}} m_{\mathrm{E} 1_{\Lambda} \hat{\imath}} .
$$

Therefore, interpreting $m_{\mathrm{D} 7_{a}}^{\hat{\imath}}$ as a matrix, $\mathrm{rk}\left(m_{\mathrm{D} 7_{a}}^{\hat{\imath}}\right)$ of the linearly independent $h_{-}^{1,1}$ E1-instantons will have chiral charged zero-modes with the D7-branes. 


\section{Conclusions}

We summarise our finding of this appendix and comment on the implication for moduli stabilisation.

- As we have seen in chapter 6, one needs $h_{+}^{1,1}$ linearly independent E3-brane instanton contributions to the non-perturbative superpotential in order to stabilise all Kähler moduli via non-perturbative effects. (Of course, for the LARGE Volume Scenario this statement is slightly modified.) In the presence of an MSSM sector, requiring a vanishing VEV for the corresponding fields, in general only

$$
\operatorname{def}\left(\mathcal{M}_{\mathrm{D} 7_{a} i}\right), \quad i=1, \ldots, h_{+}^{1,1},
$$

E3-instantons will appear in the superpotential stabilising the $T_{i}$. (Here, $\operatorname{def}(A)$ denotes again the defect of the matrix $A$.) However, in the case $h_{-}^{1,1} \neq 0$ this expression can be equal to the number of Kähler moduli $h_{+}^{1,1}$ even if chiral MSSM matter is realised.

Thus, even in the presence of an MSSM sector, all Kähler moduli can be stabilised via non-perturbative effects.

- On the other hand, in the situation $h_{-}^{1,1} \neq 0$ also the $G^{\hat{\imath}}$-moduli will be present. When considering the corresponding $h_{-}^{1,1}$ linearly independent E1-instantons, we have seen that only

$$
\operatorname{def}\left(m_{\mathrm{D} 7_{a}}^{\hat{\imath}}\right), \quad \hat{\imath}=1, \ldots, h_{-}^{1,1},
$$

of them will have no chiral charged zero-modes where $m_{\mathrm{D} 7 a}^{\hat{\imath}}$ is interpreted as a matrix.

However, the question how such instantons contribute to the superpotential and if they can be used to stabilise the $G^{\hat{\imath}}$-moduli is somewhat subtle (see for instance [65]) and will not be discussed here.

- Let us finally summarise that when the MSSM is realised by D7-branes with gauge flux, in general we have the situation that

$$
\operatorname{def}\left(\mathcal{M}_{\mathrm{D} 7_{a} i}\right)+\operatorname{def}\left(m_{\mathrm{D} 7_{a}}^{\hat{\imath}}\right)<h^{1,1}
$$

meaning that at least one of the E3- or E1-instantons has chiral charged instanton zero-modes with the MSSM branes. 
E. The Chiral Zero-Mode Constraint for $\mathbf{h}_{-}^{1,1} \neq 0$ 


\section{Bibliography}

[1] K. Simonyi, Kulturgeschichte der Physik: Von den Anfängen bis heute. Deutsch (Harri), 3. ed., December, 2001.

[2] S. Theisen, "Lecture introduction to string theory," in String Steilkurs Golm. 2004.

[3] A. Strominger, "Quantum gravity and string theory: What have we learned?," arXiv:hep-th/9110011.

[4] N. Berkovits, "New higher-derivative R**4 theorems," Phys. Rev. Lett. 98 (2007) 211601, arXiv:hep-th/0609006.

[5] Z. Bern, L. J. Dixon, and R. Roiban, "Is N = 8 Supergravity Ultraviolet Finite?," Phys. Lett. B644 (2007) 265-271, arXiv:hep-th/0611086.

[6] R. Kallosh, "N=8 Supergravity on the Light Cone," arXiv:0903.4630 [hep-th].

[7] M. B. Green, J. H. Schwarz, and E. Witten, "Superstring Theory. Vol. 1: Introduction," Cambridge, Uk: Univ. Pr. (1987) 469 P. (Cambridge Monographs On Mathematical Physics).

[8] M. B. Green, J. H. Schwarz, and E. Witten, "Superstring Theory. Vol. 2: Loop Amplitudes, Anomalies And Phenomenology,". Cambridge, Uk: Univ. Pr. (1987) 596 P. (Cambridge Monographs On Mathematical Physics).

[9] D. Lüst and S. Theisen, "Lectures on string theory," Lect. Notes Phys. 346 (1989) 1-346.

[10] J. Polchinski, "String theory. Vol. 1: An introduction to the bosonic string,". Cambridge, UK: Univ. Pr. (1998) 402 p.

[11] J. Polchinski, "String theory. Vol. 2: Superstring theory and beyond,". Cambridge, UK: Univ. Pr. (1998) 531 p. 
[12] B. Zwiebach, "A first course in string theory,". Cambridge, UK: Univ. Pr. (2004) 558 p.

[13] K. Becker, M. Becker, and J. H. Schwarz, "String theory and M-theory: A modern introduction,". Cambridge, UK: Cambridge Univ. Pr. (2007) 739 p.

[14] E. Kiritsis, "String theory in a nutshell,". Princeton, USA: Univ. Pr. (2007) 588 p.

[15] P. Ramond, "Dual Theory for Free Fermions," Phys. Rev. D3 (1971) 2415-2418.

[16] A. Neveu and J. H. Schwarz, "Factorizable dual model of pions," Nucl. Phys. B31 (1971) 86-112.

[17] F. Gliozzi, J. Scherk, and D. I. Olive, "Supergravity and the Spinor Dual Model," Phys. Lett. B65 (1976) 282.

[18] F. Gliozzi, J. Scherk, and D. I. Olive, "Supersymmetry, Supergravity Theories and the Dual Spinor Model," Nucl. Phys. B122 (1977) 253-290.

[19] M. B. Green and J. H. Schwarz, "Supersymmetrical Dual String Theory," Nucl. Phys. B181 (1981) 502-530.

[20] M. B. Green and J. H. Schwarz, "Supersymmetrical Dual String Theory. 2. Vertices and Trees," Nucl. Phys. B198 (1982) 252-268.

[21] M. B. Green and J. H. Schwarz, "Supersymmetrical String Theories," Phys. Lett. B109 (1982) 444-448.

[22] C. Vafa, "Evidence for F-Theory," Nucl. Phys. B469 (1996) 403-418, arXiv:hep-th/9602022.

[23] A. Strominger and C. Vafa, "Microscopic Origin of the Bekenstein-Hawking Entropy," Phys. Lett. B379 (1996) 99-104, arXiv: hep-th/9601029.

[24] J. M. Maldacena, "The large N limit of superconformal field theories and supergravity," Adv. Theor. Math. Phys. 2 (1998) 231-252, arXiv:hep-th/9711200.

[25] E. Witten, "Anti-de Sitter space and holography," Adv. Theor. Math. Phys. 2 (1998) 253-291, arXiv:hep-th/9802150.

[26] N. Arkani-Hamed, S. Dimopoulos, and G. R. Dvali, "The hierarchy problem and new dimensions at a millimeter," Phys. Lett. B429 (1998) 263-272, arXiv:hep-ph/9803315. 
[27] L. Randall and R. Sundrum, "A large mass hierarchy from a small extra dimension," Phys. Rev. Lett. 83 (1999) 3370-3373,

arXiv:hep-ph/9905221.

[28] L. Randall and R. Sundrum, "An alternative to compactification," Phys. Rev. Lett. 83 (1999) 4690-4693, arXiv: hep-th/9906064.

[29] G. R. Dvali, G. Gabadadze, and M. Porrati, "4D gravity on a brane in 5D Minkowski space," Phys. Lett. B485 (2000) 208-214, arXiv:hep-th/0005016.

[30] W. Lerche, D. Lüst, and A. N. Schellekens, "Chiral Four-Dimensional Heterotic Strings from Selfdual Lattices," Nucl. Phys. B287 (1987) 477.

[31] R. Bousso and J. Polchinski, "Quantization of four-form fluxes and dynamical neutralization of the cosmological constant," JHEP 06 (2000) 006, arXiv:hep-th/0004134.

[32] C. Vafa, "The string landscape and the swampland," arXiv:hep-th/0509212.

[33] D. Lüst, S. Stieberger, and T. R. Taylor, "The LHC String Hunter's Companion," Nucl. Phys. B808 (2009) 1-52, arXiv:0807.3333 [hep-th].

[34] R. Blumenhagen, M. Cvetič, P. Langacker, and G. Shiu, "Toward realistic intersecting D-brane models," Ann. Rev. Nucl. Part. Sci. 55 (2005) 71-139, arXiv:hep-th/0502005.

[35] R. Blumenhagen, B. Körs, D. Lüst, and S. Stieberger, "Four-dimensional String Compactifications with D-Branes, Orientifolds and Fluxes," Phys. Rept. 445 (2007) 1-193, arXiv:hep-th/0610327.

[36] S. Weinberg, "Implications of Dynamical Symmetry Breaking," Phys. Rev. D13 (1976) 974-996.

[37] L. Susskind, "Dynamics of Spontaneous Symmetry Breaking in the Weinberg- Salam Theory," Phys. Rev. D20 (1979) 2619-2625.

[38] H. Georgi and S. L. Glashow, "Unity of All Elementary Particle Forces," Phys. Rev. Lett. 32 (1974) 438-441.

[39] H. Fritzsch and P. Minkowski, "Unified Interactions of Leptons and Hadrons," Ann. Phys. 93 (1975) 193-266. 
[40] T. P. T. Dijkstra, L. R. Huiszoon, and A. N. Schellekens, "Supersymmetric Standard Model Spectra from RCFT orientifolds," Nucl. Phys. B710 (2005) 3-57, arXiv:hep-th/0411129.

[41] R. Blumenhagen, F. Gmeiner, G. Honecker, D. Lüst, and T. Weigand, "The statistics of supersymmetric D-brane models," Nucl. Phys. B713 (2005) 83-135, arXiv:hep-th/0411173.

[42] F. Gmeiner, R. Blumenhagen, G. Honecker, D. Lüst, and T. Weigand, "One in a billion: MSSM-like D-brane statistics," JHEP 01 (2006) 004, arXiv:hep-th/0510170.

[43] "Astrophysics and cosmology," Physics Letters B 667 (2008) no. 1-5, 212 260. Review of Particle Physics.

[44] C. D. Hoyle et al., "Sub-millimeter tests of the gravitational inverse-square law," Phys. Rev. D70 (2004) 042004, arXiv:hep-ph/0405262.

[45] J. P. Conlon, "Moduli stabilisation and applications in IIB string theory," Fortsch. Phys. 55 (2007) 287-422, arXiv: hep-th/0611039.

[46] S. Vandoren and P. van Nieuwenhuizen, "Lectures on instantons," arXiv:0802.1862 [hep-th].

[47] E. Witten, "D-branes and K-theory," JHEP 12 (1998) 019, arXiv:hep-th/9810188.

[48] A. M. Uranga, "D-brane probes, RR tadpole cancellation and K-theory charge," Nucl. Phys. B598 (2001) 225-246, arXiv:hep-th/0011048.

[49] M. B. Green and J. H. Schwarz, "Anomaly Cancellation in Supersymmetric D=10 Gauge Theory and Superstring Theory," Phys. Lett. B149 (1984) 117-122.

[50] D. S. Freed and E. Witten, "Anomalies in string theory with D-branes," arXiv:hep-th/9907189.

[51] N. Akerblom, R. Blumenhagen, D. Lüst, E. Plauschinn, and M. Schmidt-Sommerfeld, "Non-perturbative SQCD Superpotentials from String Instantons," JHEP 04 (2007) 076, arXiv:hep-th/0612132.

[52] R. Blumenhagen, S. Moster, and E. Plauschinn, "Moduli Stabilisation versus Chirality for MSSM like Type IIB Orientifolds," JHEP 01 (2008) 058, arXiv:0711.3389 [hep-th]. 
[53] E. Plauschinn, "The Generalized Green-Schwarz Mechanism for Type IIB Orientifolds with D3- and D7-Branes," arXiv:0811.2804 [hep-th].

[54] R. Blumenhagen, M. Cvetič, and T. Weigand, "Spacetime instanton corrections in $4 \mathrm{D}$ string vacua - the seesaw mechanism for D-brane models," Nucl. Phys. B771 (2007) 113-142, arXiv:hep-th/0609191.

[55] S. Kachru, R. Kallosh, A. Linde, and S. P. Trivedi, "De Sitter vacua in string theory," Phys. Rev. D68 (2003) 046005, arXiv: hep-th/0301240.

[56] V. Balasubramanian, P. Berglund, J. P. Conlon, and F. Quevedo, "Systematics of Moduli Stabilisation in Calabi-Yau Flux Compactifications," JHEP 03 (2005) 007, arXiv:hep-th/0502058

[57] J. P. Conlon, S. S. Abdussalam, F. Quevedo, and K. Suruliz, "Soft SUSY breaking terms for chiral matter in IIB string compactifications," JHEP 01 (2007) 032, arXiv:hep-th/0610129.

[58] S. B. Giddings, S. Kachru, and J. Polchinski, "Hierarchies from fluxes in string compactifications," Phys. Rev. D66 (2002) 106006. arXiv:hep-th/0105097.

[59] M. Nakahara, "Geometry, topology and physics," Boca Raton, USA: Taylor \& Francis (2003) 573 p.

[60] T. W. Grimm and J. Louis, "The effective action of $\mathrm{N}=1$ Calabi-Yau orientifolds," Nucl. Phys. B699 (2004) 387-426, arXiv:hep-th/0403067.

[61] E. Bergshoeff, R. Kallosh, T. Ortin, D. Roest, and A. Van Proeyen, "New formulations of D $=10$ supersymmetry and D8 - O8 domain walls," Class. Quant. Grav. 18 (2001) 3359-3382, arXiv:hep-th/0103233.

[62] B. Acharya, M. Aganagic, K. Hori, and C. Vafa, "Orientifolds, mirror symmetry and superpotentials," arXiv:hep-th/0202208.

[63] I. Brunner and K. Hori, "Orientifolds and mirror symmetry," JHEP 11 (2004) 005, arXiv:hep-th/0303135.

[64] T. W. Grimm, "The effective action of type II Calabi-Yau orientifolds," Fortsch. Phys. 53 (2005) 1179-1271, arXiv:hep-th/0507153.

[65] T. W. Grimm, "Axion Inflation in Type II String Theory," Phys. Rev. D77 (2008) 126007, arXiv:0710.3883 [hep-th]. 
[66] H. Jockers and J. Louis, "The effective action of D7-branes in N = 1 Calabi-Yau orientifolds," Nucl. Phys. B705 (2005) 167-211, arXiv:hep-th/0409098.

[67] H. Jockers and J. Louis, "D-terms and F-terms from D7-brane fluxes," Nucl. Phys. B718 (2005) 203-246, arXiv:hep-th/0502059.

[68] S. Kachru, M. B. Schulz, and S. Trivedi, "Moduli stabilization from fluxes in a simple IIB orientifold," JHEP 10 (2003) 007, arXiv:hep-th/0201028.

[69] A. R. Frey and J. Polchinski, "N = 3 warped compactifications," Phys. Rev. D65 (2002) 126009, arXiv: hep-th/0201029.

[70] K. Dasgupta, G. Rajesh, and S. Sethi, "M theory, orientifolds and G-flux," JHEP 08 (1999) 023, arXiv: hep-th/9908088.

[71] M. Marino, R. Minasian, G. W. Moore, and A. Strominger, "Nonlinear instantons from supersymmetric p-branes," JHEP 01 (2000) 005, arXiv:hep-th/9911206.

[72] R. Blumenhagen, V. Braun, T. W. Grimm, and T. Weigand, "GUTs in Type IIB Orientifold Compactifications," arXiv:0811.2936 [hep-th].

[73] E. S. Fradkin and A. A. Tseytlin, "Nonlinear Electrodynamics from Quantized Strings," Phys. Lett. B163 (1985) 123.

[74] R. G. Leigh, "Dirac-Born-Infeld Action from Dirichlet Sigma Model," Mod. Phys. Lett. A4 (1989) 2767.

[75] R. C. Myers, "Dielectric-branes," JHEP 12 (1999) 022, arXiv:hep-th/9910053.

[76] M. R. Douglas, "Branes within branes," arXiv:hep-th/9512077.

[77] M. B. Green, J. A. Harvey, and G. W. Moore, "I-brane inflow and anomalous couplings on D-branes," Class. Quant. Grav. 14 (1997) 47-52, arXiv:hep-th/9605033.

[78] Y.-K. E. Cheung and Z. Yin, "Anomalies, branes, and currents," Nucl. Phys. B517 (1998) 69-91, arXiv:hep-th/9710206.

[79] J. F. Morales, C. A. Scrucca, and M. Serone, "Anomalous couplings for D-branes and O-planes," Nucl. Phys. B552 (1999) 291-315, arXiv:hep-th/9812071. 
[80] J. Stefanski, Bogdan, "Gravitational couplings of D-branes and O-planes," Nucl. Phys. B548 (1999) 275-290, arXiv:hep-th/9812088.

[81] C. A. Scrucca and M. Serone, "Anomalies and inflow on D-branes and O-planes," Nucl. Phys. B556 (1999) 197-221, arXiv:hep-th/9903145.

[82] R. Blumenhagen, G. Honecker, and T. Weigand, "Non-abelian brane worlds: The open string story," arXiv:hep-th/0510050.

[83] S. J. Gates, M. T. Grisaru, M. Rocek, and W. Siegel, "Superspace, or one thousand and one lessons in supersymmetry," Front. Phys. 58 (1983) 1-548, arXiv:hep-th/0108200.

[84] J. Wess and J. Bagger, "Supersymmetry and supergravity,". Princeton, USA: Univ. Pr. (1992) 259 p.

[85] H. Jockers, "The effective action of D-branes in Calabi-Yau orientifold compactifications," Fortsch. Phys. 53 (2005) 1087-1175, arXiv:hep-th/0507042.

[86] A. Strominger, "Special Geometry," Commun. Math. Phys. 133 (1990) 163-180.

[87] P. Candelas and X. de la Ossa, "Moduli Space Of Calabi-Yau Manifolds," Nucl. Phys. B355 (1991) 455-481.

[88] K. Becker, M. Becker, M. Haack, and J. Louis, "Supersymmetry breaking and alpha'-corrections to flux induced potentials," JHEP 06 (2002) 060, arXiv:hep-th/0204254.

[89] M. Berg, M. Haack, and B. Körs, "String loop corrections to Kaehler potentials in orientifolds," JHEP 11 (2005) 030, arXiv:hep-th/0508043.

[90] M. Berg, M. Haack, and B. Körs, "On volume stabilization by quantum corrections," Phys. Rev. Lett. 96 (2006) 021601, arXiv:hep-th/0508171.

[91] M. Berg, M. Haack, and E. Pajer, "Jumping Through Loops: On Soft Terms from Large Volume Compactifications," JHEP 09 (2007) 031, arXiv:0704.0737 [hep-th].

[92] M. Cicoli, J. P. Conlon, and F. Quevedo, "Systematics of String Loop Corrections in Type IIB Calabi- Yau Flux Compactifications," JHEP 01 (2008) 052, arXiv:0708.1873 [hep-th]. 
[93] K. Hristov, "Axion Stabilization in Type IIB Flux Compactifications," JHEP 01 (2009) 046, arXiv:0810.3329 [hep-th].

[94] S. Gukov, C. Vafa, and E. Witten, "CFT's from Calabi-Yau four-folds," Nucl. Phys. B584 (2000) 69-108, arXiv:hep-th/9906070.

[95] T. R. Taylor and C. Vafa, "RR flux on Calabi-Yau and partial supersymmetry breaking," Phys. Lett. B474 (2000) 130-137, arXiv:hep-th/9912152.

[96] O. DeWolfe and S. B. Giddings, "Scales and hierarchies in warped compactifications and brane worlds," Phys. Rev. D67 (2003) 066008, arXiv:hep-th/0208123.

[97] F. Marchesano, "Progress in D-brane model building," Fortsch. Phys. 55 (2007) 491-518, arXiv:hep-th/0702094.

[98] M. F. Atiyah and I. M. Singer, "The Index of elliptic operators. 1," Annals Math. 87 (1968) 484-530.

[99] M. F. Atiyah and G. B. Segal, "The Index of elliptic operators. 2," Annals Math. 87 (1968) 531-545.

[100] M. F. Atiyah and I. M. Singer, "The Index of elliptic operators. 3," Annals Math. 87 (1968) 546-604.

[101] A. Sagnotti, "A Note on the Green-Schwarz mechanism in open string theories," Phys. Lett. B294 (1992) 196-203, arXiv: hep-th/9210127.

[102] G. Aldazabal, A. Font, L. E. Ibáñez, and G. Violero, "D =4, N =1, type IIB orientifolds," Nucl. Phys. B536 (1998) 29-68, arXiv: hep-th/9804026.

[103] L. E. Ibáñez, R. Rabadan, and A. M. Uranga, "Anomalous U(1)'s in type I and type IIB D =4, N = 1 string vacua," Nucl. Phys. B542 (1999) 112-138, arXiv:hep-th/9808139.

[104] L. E. Ibáñez, R. Rabadan, and A. M. Uranga, "Sigma-model anomalies in compact $\mathrm{D}=4, \mathrm{~N}=1$ type IIB orientifolds and Fayet-Iliopoulos terms," Nucl. Phys. B576 (2000) 285-312, arXiv:hep-th/9905098.

[105] G. Aldazabal, S. Franco, L. E. Ibáñez, R. Rabadan, and A. M. Uranga, "D $=4$ chiral string compactifications from intersecting branes," J. Math. Phys. 42 (2001) 3103-3126, arXiv: hep-th/0011073.

[106] E. Witten, "An SU(2) anomaly," Phys. Lett. B117 (1982) 324-328. 
[107] R. Minasian and G. W. Moore, "K-theory and Ramond-Ramond charge," JHEP 11 (1997) 002, arXiv:hep-th/9710230.

[108] S. H. Katz and E. Sharpe, "D-branes, open string vertex operators, and Ext groups," Adv. Theor. Math. Phys. 6 (2003) 979-1030, arXiv:hep-th/0208104.

[109] P. S. Aspinwall, "D-branes on Calabi-Yau manifolds," arXiv:hep-th/0403166.

[110] R. Blumenhagen, M. Cvetič, S. Kachru, and T. Weigand, "D-brane Instantons in Type II String Theory," arXiv:0902.3251 [hep-th].

[111] R. Blumenhagen, M. Cvetič, R. Richter, and T. Weigand, "Lifting d-instanton zero modes by recombination and background fluxes," arXiv:0708.0403 [hep-th].

[112] R. Argurio, M. Bertolini, S. Franco, and S. Kachru, "Metastable vacua and D-branes at the conifold," JHEP 06 (2007) 017, arXiv:hep-th/0703236.

[113] R. Argurio, M. Bertolini, G. Ferretti, A. Lerda, and C. Petersson, "Stringy Instantons at Orbifold Singularities," JHEP 06 (2007) 067, arXiv:0704.0262 [hep-th].

[114] M. Bianchi, F. Fucito, and J. F. Morales, "D-brane instantons on the $t^{6} / z_{3}$ orientifold," JHEP 07 (2007) 038, arXiv:0704.0784 [hep-th].

[115] L. E. Ibáñez, A. N. Schellekens, and A. M. Uranga, "Instanton Induced Neutrino Majorana Masses in CFT Orientifolds with MSSM-like spectra," JHEP 06 (2007) 011, arXiv:0704.1079 [hep-th].

[116] J. Polchinski, "Combinatorics of boundaries in string theory," Phys. Rev. D50 (1994) 6041-6045, arXiv:hep-th/9407031.

[117] M. F. Atiyah, N. J. Hitchin, V. G. Drinfeld, and Y. I. Manin, "Construction of instantons," Phys. Lett. A65 (1978) 185-187.

[118] M. B. Green and M. Gutperle, "Effects of D-instantons," Nucl. Phys. B498 (1997) 195-227, arXiv: hep-th/9701093.

[119] M. B. Green and M. Gutperle, "D-particle bound states and the D-instanton measure," JHEP 01 (1998) 005, arXiv:hep-th/9711107.

[120] M. Billò et al., "Classical gauge instantons from open strings," JHEP 02 (2003) 045, arXiv:hep-th/0211250. 
[121] C. Petersson, "Superpotentials From Stringy Instantons Without Orientifolds," JHEP 05 (2008) 078, arXiv:0711.1837 [hep-th].

[122] M. Billò et al., "Flux interactions on D-branes and instantons," JHEP 10 (2008) 112, arXiv:0807.1666 [hep-th].

[123] M. Grana, "Flux compactifications in string theory: A comprehensive review," Phys. Rept. 423 (2006) 91-158, arXiv:hep-th/0509003.

[124] M. R. Douglas and S. Kachru, "Flux compactification," Rev. Mod. Phys. 79 (2007) 733-796, arXiv:hep-th/0610102.

[125] F. Denef, M. R. Douglas, and S. Kachru, "Physics of string flux compactifications," Ann. Rev. Nucl. Part. Sci. 57 (2007) 119-144, arXiv:hep-th/0701050.

[126] Supernova Cosmology Project Collaboration, S. Perlmutter et al., "Measurements of Omega and Lambda from 42 High-Redshift Supernovae," Astrophys. J. 517 (1999) 565-586, arXiv:astro-ph/9812133.

[127] Supernova Search Team Collaboration, A. G. Riess et al., "Observational Evidence from Supernovae for an Accelerating Universe and a Cosmological Constant," Astron. J. 116 (1998) 1009-1038, arXiv:astro-ph/9805201.

[128] I. R. Klebanov and M. J. Strassler, "Supergravity and a confining gauge theory: Duality cascades and chiSB-resolution of naked singularities," JHEP 08 (2000) 052, arXiv:hep-th/0007191.

[129] M. R. Douglas, "The statistics of string/M theory vacua," JHEP 05 (2003) 046, arXiv:hep-th/0303194.

[130] K. Choi, A. Falkowski, H. P. Nilles, M. Olechowski, and S. Pokorski, "Stability of flux compactifications and the pattern of supersymmetry breaking," JHEP 11 (2004) 076, arXiv:hep-th/0411066.

[131] S. P. de Alwis, "Effective potentials for light moduli," Phys. Lett. B626 (2005) 223-229, arXiv:hep-th/0506266.

[132] K. Choi, A. Falkowski, H. P. Nilles, and M. Olechowski, "Soft supersymmetry breaking in KKLT flux compactification," Nucl. Phys. B718 (2005) 113-133, arXiv:hep-th/0503216. 
[133] L. Covi, M. Gomez-Reino, C. Gross, G. A. Palma, and C. A. Scrucca, "Constructing de Sitter vacua in no-scale string models without uplifting," arXiv:0812.3864 [hep-th].

[134] J. P. Conlon, F. Quevedo, and K. Suruliz, "Large-volume flux compactifications: Moduli spectrum and D3/D7 soft supersymmetry breaking," JHEP 08 (2005) 007, arXiv:hep-th/0505076.

[135] F. Denef, M. R. Douglas, and B. Florea, "Building a better racetrack," JHEP 06 (2004) 034, arXiv:hep-th/0404257.

[136] G. Curio and V. Spillner, "On the modified KKLT procedure: A case study for the P(11169)(18) model," Int. J. Mod. Phys. A22 (2007) 3463-3492, arXiv:hep-th/0606047.

[137] A. Misra and P. Shukla, "Area Codes, Large Volume (Non-)Perturbative alpha'- and Instanton - Corrected Non-supersymmetric (A)dS minimum, the Inverse Problem and Fake Superpotentials for MultipleSingular-Loci-Two-Parameter Calabi-Yau's," Nucl. Phys. B799 (2008) 165-198, arXiv:0707.0105 [hep-th].

[138] A. Collinucci, M. Kreuzer, C. Mayrhofer, and N.-O. Walliser, "Four-modulus 'Swiss Cheese' chiral models," arXiv:0811.4599 [hep-th].

[139] M. Cicoli, J. P. Conlon, and F. Quevedo, "General Analysis of LARGE Volume Scenarios with String Loop Moduli Stabilisation," JHEP 10 (2008) 105, arXiv:0805.1029 [hep-th].

[140] C. P. Burgess et al., "Continuous Global Symmetries and Hyperweak Interactions in String Compactifications," JHEP 07 (2008) 073, arXiv:0805.4037 [hep-th].

[141] L. Anguelova, V. Calo, and M. Cicoli, "LARGE Volume String Compactifications at Finite Temperature," arXiv:0904.0051 [hep-th].

[142] J. P. Conlon, "Gauge Threshold Corrections for Local String Models," arXiv:0901.4350 [hep-th].

[143] J. P. Conlon, C. H. Kom, K. Suruliz, B. C. Allanach, and F. Quevedo, "Sparticle Spectra and LHC Signatures for Large Volume String Compactifications," JHEP 08 (2007) 061, arXiv:0704.3403 [hep-ph].

[144] A. Sen, "F-theory and Orientifolds," Nucl. Phys. B475 (1996) 562-578, arXiv:hep-th/9605150. 
[145] A. Collinucci, F. Denef, and M. Esole, "D-brane Deconstructions in IIB Orientifolds," arXiv:0805.1573 [hep-th].

[146] A. P. Braun, A. Hebecker, and H. Triendl, "D7-Brane Motion from M-Theory Cycles and Obstructions in the Weak Coupling Limit," Nucl. Phys. B800 (2008) 298-329, arXiv:0801.2163 [hep-th].

[147] P. Aluffi and M. Esole, "Chern class identities from tadpole matching in type IIB and F-theory," arXiv:0710.2544 [hep-th].

[148] S. Sethi, C. Vafa, and E. Witten, "Constraints on low-dimensional string compactifications," Nucl. Phys. B480 (1996) 213-224, arXiv:hep-th/9606122.

[149] F. G. Marchesano Buznego, "Intersecting D-brane models," arXiv:hep-th/0307252.

[150] I. Affleck, M. Dine, and N. Seiberg, "Dynamical Supersymmetry Breaking in Supersymmetric QCD," Nucl. Phys. B241 (1984) 493-534.

[151] N. Akerblom, "D-instantons and effective couplings in intersecting Dbrane models," Fortsch. Phys. 56 (2008) 1065-1142.

[152] K. Becker, M. Becker, and A. Strominger, "Five-branes, membranes and nonperturbative string theory," Nucl. Phys. B456 (1995) 130-152, arXiv:hep-th/9507158.

[153] M. Haack, D. Krefl, D. Lüst, A. Van Proeyen, and M. Zagermann, "Gaugino condensates and D-terms from D7-branes," JHEP 01 (2007) 078, arXiv:hep-th/0609211.

[154] J. P. Conlon, "The QCD axion and moduli stabilisation," JHEP 05 (2006) 078, arXiv:hep-th/0602233.

[155] J. P. Conlon and F. Quevedo, "Gaugino and scalar masses in the landscape," JHEP 06 (2006) 029, hep-th/0605141.

[156] J. P. Conlon and D. Cremades, "The neutrino suppression scale from large volumes," Phys. Rev. Lett. 99 (2007) 041803, hep-ph/0611144. 